\title{
Understanding the generation and regulation of IL-4 producing T helper 2 cells.
}

\author{
Helen Mearns \\ A thesis submitted for the degree of Doctor of Philosophy \\ Victoria University of Wellington, New Zealand \\ Malaghan Institute of Medical Research
}

April 2012 



\begin{abstract}
:
A keenly sought therapeutic approach for the treatment of allergic disease is the identification and neutralization of the cytokine that regulates the differentiation of Th2 cells. Th2 cells are CD4 T cells differentiated to secrete IL-4. These cells are exciting new targets for asthma therapies due to the key role they play in allergic airway diseases. Recently the cytokine IL-25 has been shown to enhance Th2 cell activity and play important roles in mediating allergic inflammatory responses. To investigate this further we crossed IL-25 deficient mice with GFP-IL-4 reporter mice and developed an assay of in vitro and in vivo IL-4 independent Th2 differentiation. These assays were used to determine whether IL-25 was critical for the formation of Th2 cells. We found there was no physiological role for IL-25 in either the differentiation of Th2 cells or their development to effector or memory Th2 subsets. In the strong Th2 setting of a helminth infection the absence of IL-25 resulted in no defects in the effector type 2 responses associated with $\mathrm{T}$ helper type 2 immunity including, mucous hyperplasia, class switching to $\operatorname{IgE}$ and protection against challenge infections. Importantly this data challenges the newly found and growing status of the cytokine IL-25 and its proposed role in promoting Th2 responses.
\end{abstract}

The second part of this thesis investigated whether the genomic organisation, which reflects commitment to Th2 cytokine expression, could provide a clearer definition of a functional in vivo Th2 cell. Two distinct IL-4 reporter mice were crossed and Th2 in vivo assays were developed that allowed tracking of the individual alleles of IL-4 in a variety of tissue types and Th2 subsets. Interestingly in vivo expression of IL-4 is mostly monoallelic yet there is a small highly activated population of biallelic IL-4 expressing Th2 cells. Physiologically each allele of IL-4 was required for a functional Th2 response with total serum IgE titres up to 4 fold lower in $\mathrm{IL}_{-} 4^{+/-}$heterozygous compared to the $\mathrm{IL}_{-} 4^{+/+}$sufficient animals and a significant loss in protective immunity against challenge infections with helminths occurred in the IL- $4^{+/}$heterozygous animals. The similarity in deficiencies in Th2 immunity in the $\mathrm{IL}-4^{+/-}$heterozygous and $\mathrm{IL}-4^{-/-}$deficient compared to the $\mathrm{IL}-4^{+/+}$ sufficient animals lead to the proposal that the generation of biallelic Th2 cells may 
be required for specialised cell-to-cell mediated delivery of tailored activation signals and higher quantities of IL-4 required to mediate fully developed Th2 immune responses. 


\section{Acknowledgements:}

Firstly I wish to acknowledge the generosity of my supervisor Graham Le Gros, I think the amount of energy, thought and dedication he has to both his current and past lab members is a credit to his successful mentorship and engaging style. I will always have fond memories of our very animated debates. I would also like to acknowledge Franca Ronchese whose door was always open for discussion and direction. I would like to thank Liz Forbes-Blom for helping me keep up with the never ending literature, being great for discussing ideas with, assisting with ELISpot and ELISA data generation and generously giving hours of her time to provide extremely helpful feedback on thesis drafts. I would like to thank the GLG lab members both past and present but most especially Mali Camberis, Shiau-Choot Tang, Mel Prout, for their tireless assistance in the lab and sharing of much appreciated expert knowledge. Marcus Robinson for collaborating on the analysis of regulation of alleles of IL-4 project and Ryan Kyle for assistance with Bioplex of mesenteric lymph node samples. I would also like to thank my fellow Malaghanites for making this endeavour successful. With special thanks to Haley Ataera for invaluable thesis feedback and never ending encouragement, Lisa Connor for some great editing advice, Brigitta Mester and Kylie Price for being so lovely and supportive and sharing their amazing skills in the flow lab. I wish to acknowledge the generous funding granted to me from the Health Research Council of New Zealand as well as travel grants from the Wellington Medical Research Council, the School of Biological Science, the National Institutes of Health travel grant for the Woods Hole Immunoparasitology Meeting and the Submission Scholarship from the Victoria University of Wellington. I would also like to thank the generous donations by Melanie Kleinschek (SP Biopharma, USA) of IL-25 deficient mice, Bill Paul (NIAD, NIH, USA) for the G4 reporter mice and Richard Locksley (UCSF, USA) for the KN2 reporter mice. I would also like to thank Bill Horsnell who through the years has continued to support me and encourage me to challenge my limits. Last but certainly not least I would like to thank my family and friends, without whom there would be little point in any of this, you guys are the sweet before as and the choice before bro, you're even better than a chop on a braai! 


\title{
Table of Contents:
}

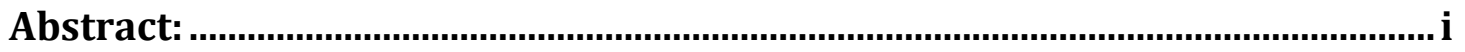

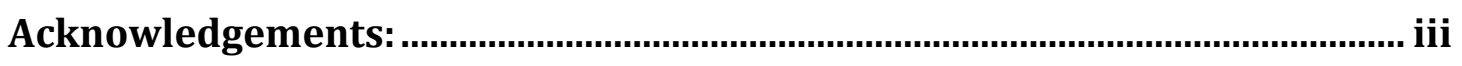

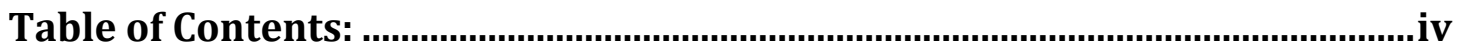

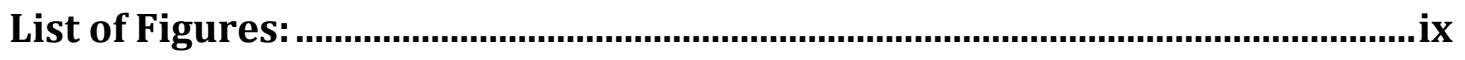

List of Tables:

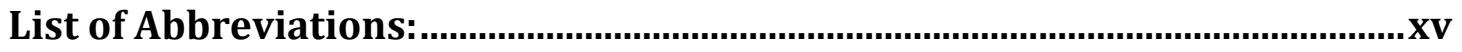

Chapter One:

General Introduction
\end{abstract}

1. 1 Subsets of CD4 T helper cells and their involvement in immunity ....................2

1. 2 Interleukin-4: The signature cytokine of a T helper 2 cell...................................5

1. 3 Requirements for the differentiation of a $\operatorname{Th} 2$ cell ............................................5

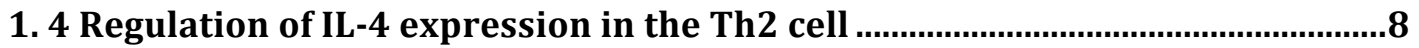

1. 5 Regulation of expression of IL-4 in a T follicular helper (Tfh) cell ..................11

1. 6 Regulation of class-switch recombination of B cells to IgE by IL-4 producing

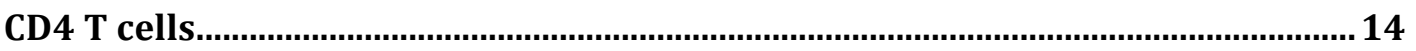

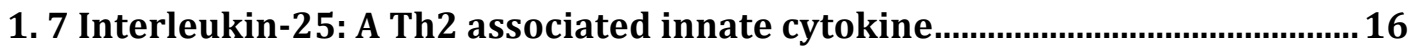

1. 8 IL-4 mediated immune responses to parasitic helminths.................................... 18

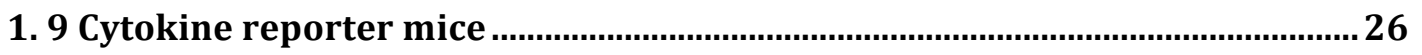

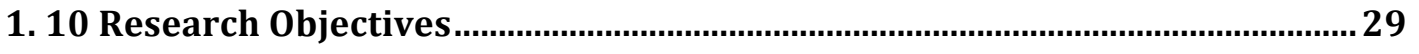

Research Objective 1:...........................................................................................................29

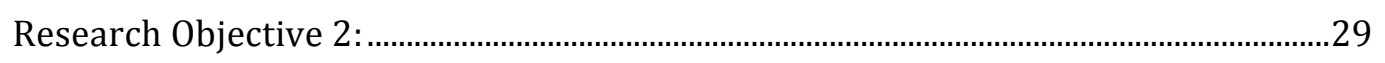

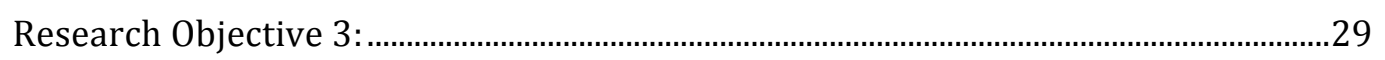

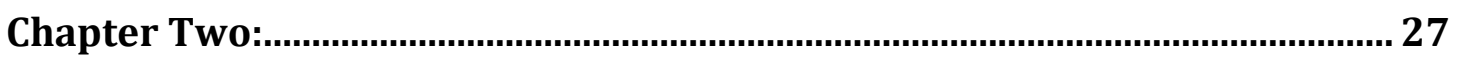

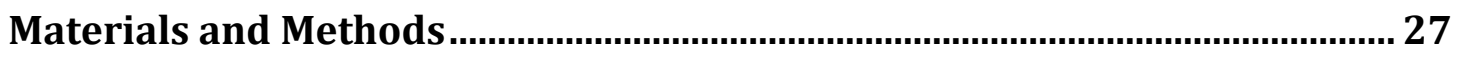

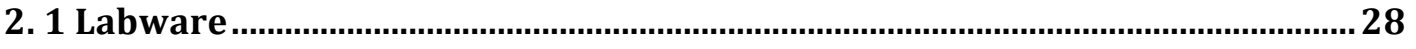

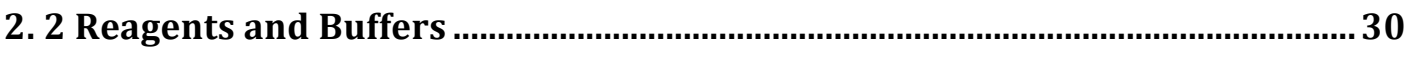

2.2. 1 2-mercaptoethanol (2 ME) ................................................................................

2.2. 2 Alsever's Solution ..................................................................................................... 


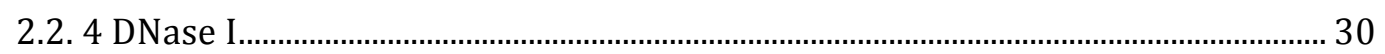

2.2. 5 Dimethyl sulfoxide (DMSO) ................................................................................... 30

2.2. 6 Ethylenediaminetetraacetic acid (EDTA) …………………………………............... 30

2.2. 7 Fluorescence Activated Cell Sorting (FACs) Buffer................................................ 31

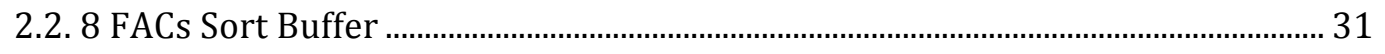

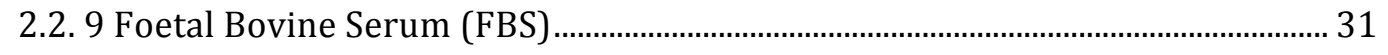

2.2. 10 Complete Iscove's Modified Dulbecco's Medium (cIMDM)................................. 31

2.2. 11 Lipopolysaccharides (LPS)................................................................................. 31

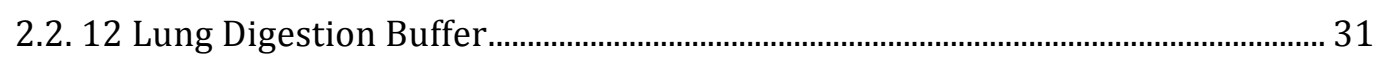

2.2. 13 Magnetic Cell Sorting (MACS) Running Buffer ..................................................... 31

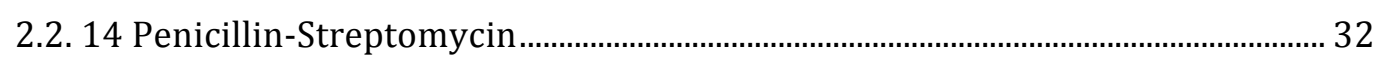

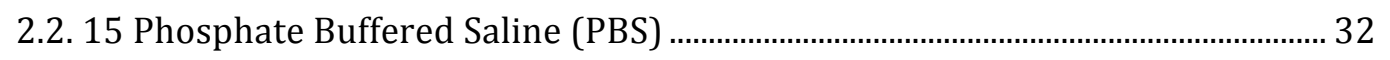

2.2. 16 Red Blood Cell Lysing Buffer Hybri-Max ${ }^{\mathrm{m}}$ (RBC) ………………......................... 32

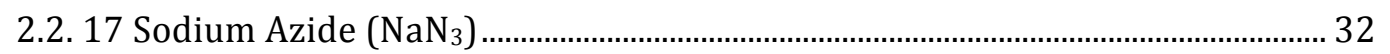

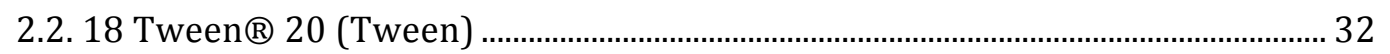

2.2. 19 Trypan Blue Stain (Life Technologies ${ }^{\mathrm{TM}}$ ) ............................................................. 32

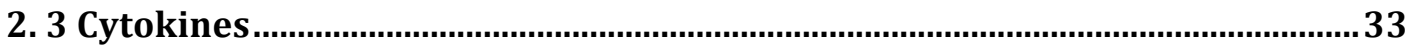

2.3. 1 Fms-like tyrosine kinase ligand (rFlt3L) ……......................................................... 33

2.3. 2 Granulocyte-macrophage colony stimulating factor (rGM-CSF) .......................... 33

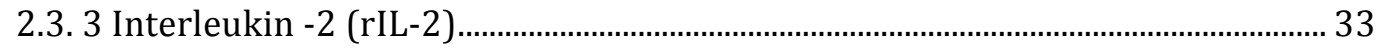

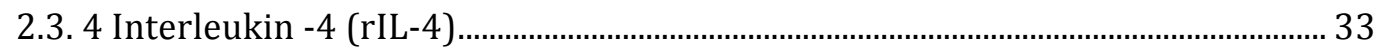

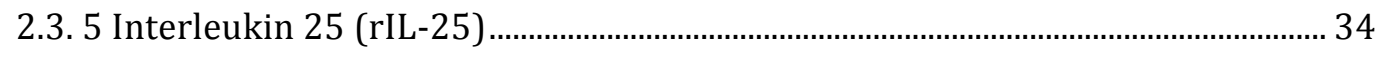

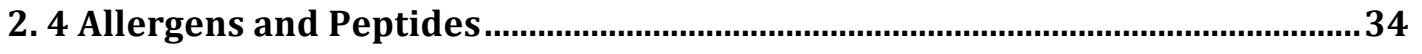

2.4. 1 ISQ peptide

2.4. 2 House Dust Mite (HDM) ........................................................................................ 34

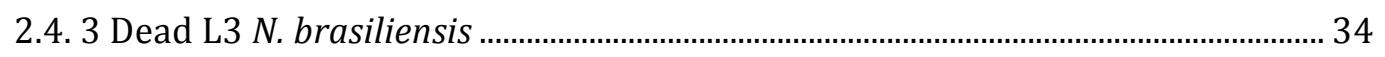

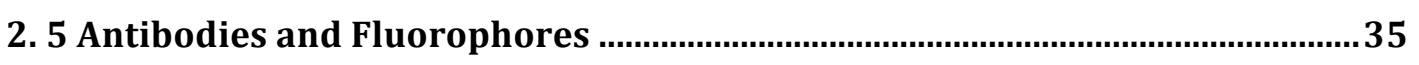

2.5. 1 4',6-diamidino-2-phenylindole, dihydrochloride (DAPI) ...................................... 35

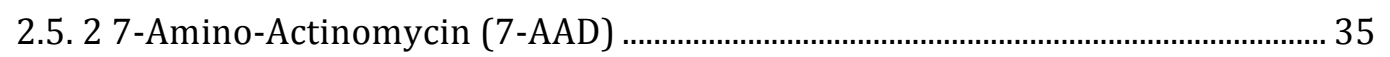

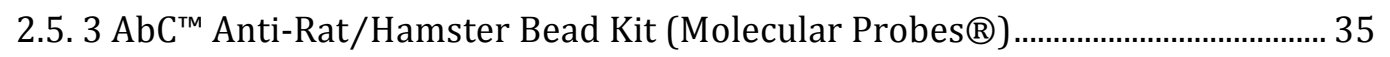

2.5. 4 Anti-Mouse Ig, к/Negative Control (FBS) Compensation Particles Set............. 35

2.5. 5 LIVE/DEAD® Fixable Blue Dead Cell Stain Kit (Molecular Probes $®$ ) ............... 36

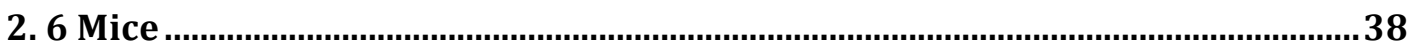

2.6. 1 Maintenance and Ethics Approvals............................................................................... 38

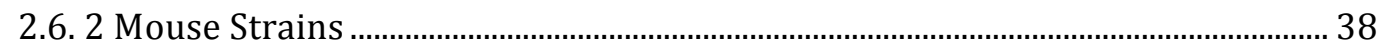




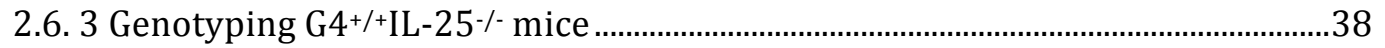

2.6. 4 Genotyping OTII and OTII G4+/+ mice …………….....................................................40

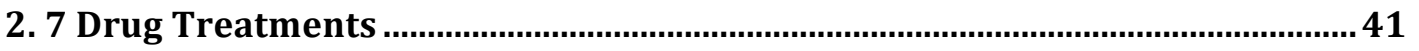

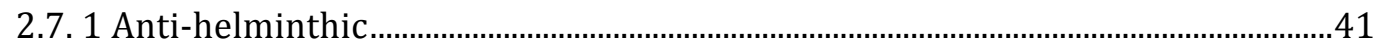

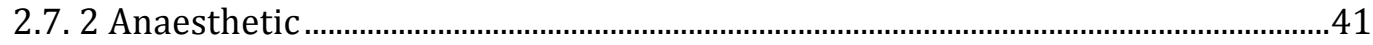

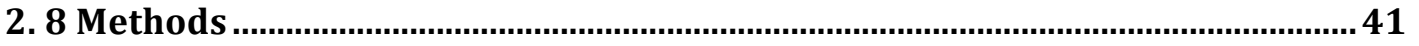

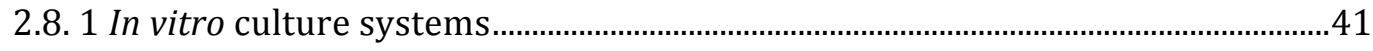

2.8. 2 In vivo assay for differentiation of Th2 cells ......................................................... 43

2.8. 3 FACS analysis and staining .........................................................................................

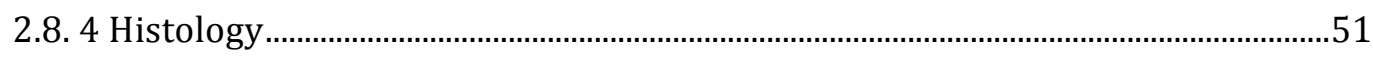

2.8. 5 Enzyme-linked Immunosorbent assay (ELISA)....................................................51

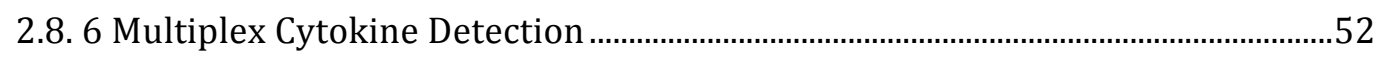

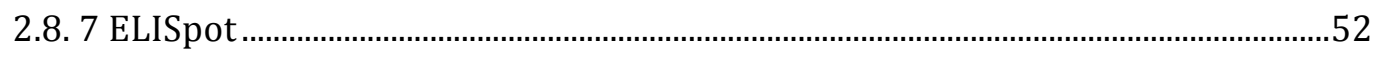

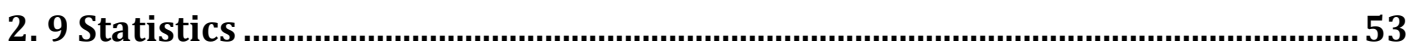

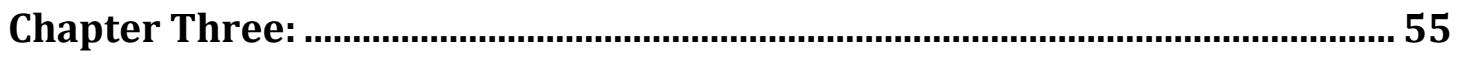

Investigating the potential polarising role of IL-25 during the

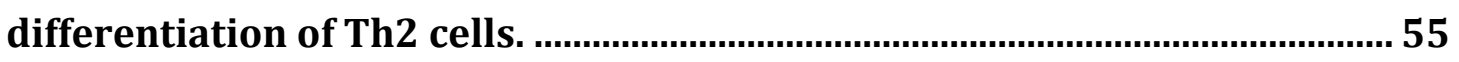

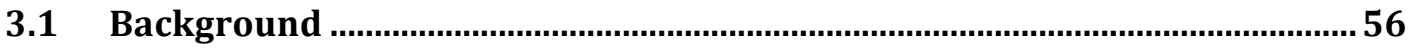

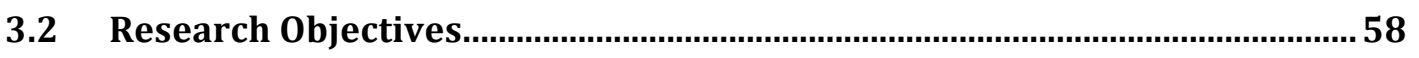

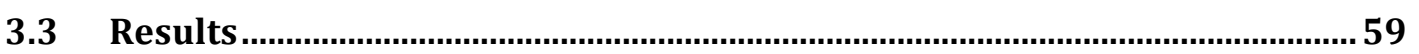

3.3. 1 Optimisation of the in vitro assay for IL-4 independent differentiation of Th2 cells: Polyclonal differentiation.................................................................................59

3.3. 2 Optimisation of the in vitro assay for IL-4 independent differentiation of Th2 cells: Titrating activation stimulus..

3.3. 3 Optimisation of the in vitro assay for IL-4 independent differentiation of Th2 cells: TCR expression in transgenic mice does not alter Th2 responses to polyclonal activation.

3.3. 4 Optimisation of the in vitro assay for IL-4 independent differentiation of Th2 cells: Confirming TCR expression in transgenic mice.

3.3. 5 Optimisation of the in vitro assay for IL-4 independent differentiation of Th2 cells: APC to T cell ratio for maximal Th2 cell differentiation. . .65

3.3. 6 Optimisation of the in vitro assay for IL-4 independent differentiation of Th2 cells: IL-4 present in BMDC culture does not cross-contaminate T cell culture. 66 
3.3. 7 Optimisation of the in vitro assay for IL-4 independent differentiation of Th2 cells: Maturing BMDCs with rIL-25.

3.3. 8 Optimisation of the in vitro assay for IL-4 independent differentiation of Th2 cells: Flt3L generated BMDCs.

3.3. 9 Optimisation of the in vitro assay for IL-4 independent differentiation of Th2 cells: Altering antigen concentration to promote Th2 differentiation... 72

3.3. 10 Investigating the role of IL-25 as an early differentiation factor in the antigen specific in vitro assay of IL-4 independent Th2 differentiation. . 75

3.3. 11 Optimisation of the in vivo assay for differentiation of Th2 cells using worm derived allergens.

3.3.12 Optimisation of the in vivo assay for differentiation of Th2 cells using worm derived allergens: Determining expression of Th2 associated cytokines........ 76 3.3. 13 Using the in vivo assay for $\mathrm{CD} 4 \mathrm{~T}$ cell differentiation to investigate the role of IL-25 in Th2 differentiation........................................................................................... 78

3.4 Discussion .80

Chapter Four: 83

The role of IL-25 in the generation of protective T helper type 2 responses against infection by parasitic helminths........................................................... 83

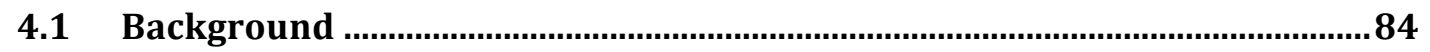

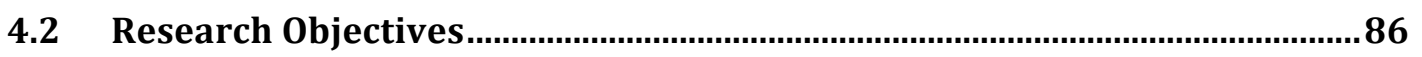

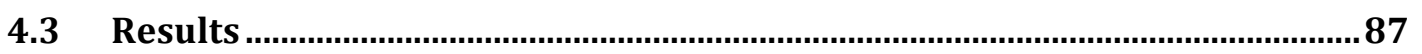

4.3. 1 Helminth induced Th2 cells are not altered by the absence of IL-25 ............ 87

4.3. 2 Type 2 cytokine profile in the lung is not altered by the lack of IL-25......... 90

4.3. 3 Isotype switching to IgE is unaffected by loss of IL-25 ................................... 91

4.3. 4 Basophilia, eosinophilia and goblet cell hyperplasia continue unimpeded in the absence of IL-25 signalling. 92

4.3. 5 Loss of IL-25 has no effect on the nuocyte populations detectable in lungs or mediastinal lymph nodes

4.3. 6 Lung protective immunity to $N$. brasiliensis continues in the absence of IL25 signalling .106

4.3. 7 Th2 cells, type 2 cytokines and nuocytes in the mesenteric lymph node show no deficiency in the absence of IL-25.

4.3. 8 H. polygyrus expulsion from the gut is delayed in IL-25 deficient animals113

4.4 Discussion 116

Chapter Five:. 123 
Characterisation of a Th2 cell: The allelic expression of IL-4.

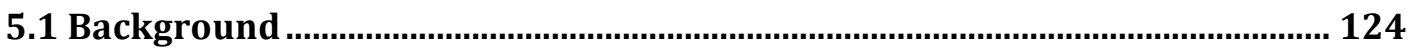

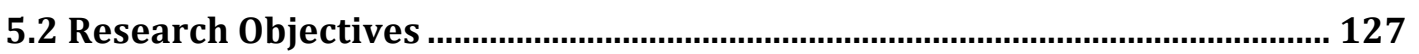

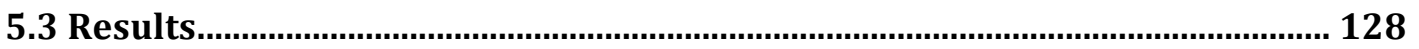

5.3. 1 Monoallelic expression of IL-4 is the predominant form of regulation in both effector and memory Th2 cells ...................................................................................128

5.3. 2 Th2 cells with biallelic IL-4 expression have a higher activation status than Th2 cells with monoallelic IL-4 expression

5.3. 3 Th2 cells in lymphoid and tissue environments have similar monoallelic dominant patterns of IL-4 allelic expression

5.3. 4 Functional relevance of both alleles of IL-4 in protective immunity against parasitic helminth infections 136

5.3. 5 Functional relevance of both alleles of IL-4 in generating full lymphocyte compartments of the $\mathrm{Th} 2$ response

5.3. 6 T helper cells with biallelic IL-4 expression are more likely to be Tfh cells than the T helper cells with monoallelic IL-4 expression.

5.3. 7 The loss of a single allele of IL-4 compromises the numbers of IL-4 producing CD4 T cells.

5.3. 8 Role of IL- 4 in the induction of Th2 and Tfh transcription factors GATA3 and BCL6 in CD4 T cells.

5.4 Discussion 151

Chapter Six: 157

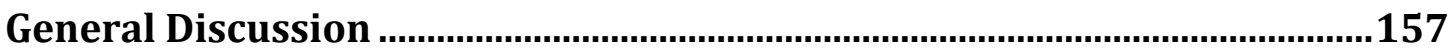

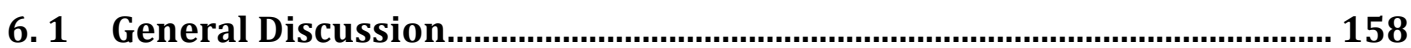

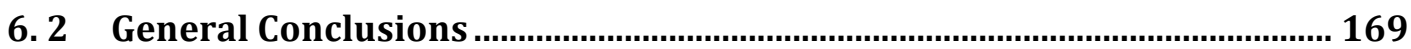

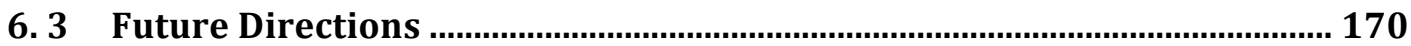

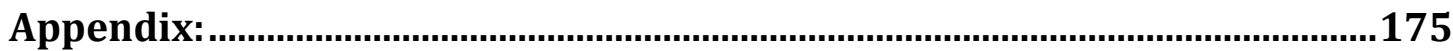

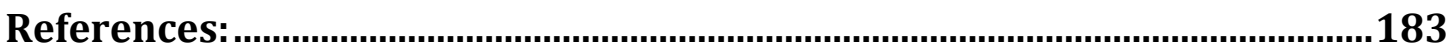




\section{List of Figures:}

Figure 1. 1 Diagram illustrating the four well characterised T helper subsets............. 4

Figure 1. 2 Fundamental signals involved in Th2 differentiation............................... 7

Figure 1. 3 Pathways of $\mathrm{T}$ helper 2 cell differentiation, both IL-4 dependent and independent, require activation of GATA-binding protein 3 ........................... 10

Figure 1. 4 Photomicrograph of a rat spleen section showing a well-developed germinal centre at the height of the germinal centre reaction. 12

Figure 1. 5 The differentiated Th2 cell population comprises multiple functionally distinct subsets. 16

Figure 1. 6 Potential roles for IL-25 during differentiation and development of a Th2 immune response. 18

Figure 1. 7 Expulsion of primary Nippostrongylus brasiliensis is dependent upon Th2 mediated immunity. 22

Figure 1.8 Memory Th2 responses to Nippostrongylus brasiliensis are generated in the lung..... 24

Figure 1. 9 Protective immunity against challenge infections by Heligmosomoides polygyrus is dependent upon Th2 cells and alternatively activated macrophages (AAMacs). 26

Figure 2. 1 Genotyping of IL-4 reporter mice ${ }^{+/} \mathrm{IL}-25^{-/-}$mice. 40

Figure 3. 1 Polyclonal activation of CD4 T cells differentiates Th2 cells even in the absence of IL-4. Positively selected naïve CD4 T cells from spleens were incubated on plate bound $\alpha \mathrm{CD} 3(10 \mu \mathrm{g} / \mathrm{ml})$, soluble $\alpha \mathrm{CD} 28$ (1:50) and rIL-2 (50 $\mathrm{U} / \mathrm{ml})$ with or without rIL-4 $(1000 \mathrm{U} / \mathrm{ml})$ for up to 5 days. 60

Figure 3. 2 Polyclonal activation of CD4 T cells requires sufficient concentrations of $\alpha \mathrm{CD} 3$ for proliferation and Th2 differentiation.

Figure 3. 3 Polyclonal activation of TCR Tg OTII G4 ${ }^{+/+}$CD4 T cells did not result in non-specific GFP production. 64

Figure 3. 4 The majority of CD4 $\mathrm{T}$ cells in TCR Tg OTII $\mathrm{G}^{+/+}$mice express both transgenic $\alpha$ and $\beta$ chains. 65

Figure 3. $5 \mathrm{BMDC}$ to $\mathrm{CD} 4 \mathrm{~T}$ cell ratio required for optimal cell counts and $\mathrm{Th} 2$ induction. 
Figure 3. 6 IL-4 from the GMCSF/IL-4 BMDC culture is not responsible for

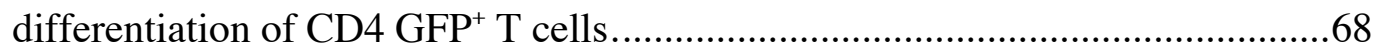

Figure 3. 7 Maturation of BMDCs for initiation of Th2 differentiation in vitro...........70

Figure 3. 8 Lack of frequency of Th2 cells by Flt3L DCs is not as a result of excessive production of IL-12 and Th1 differentiation....................................................71

Figure 3. 9 Decreased antigen concentration results in increased $\mathrm{Th} 2$ frequency.......73

Figure 3. 10 Low concentrations of antigen are crucial for high Th2 frequencies. .....74

Figure 3. 11 Neither exogenous nor bone marrow dendritic cell derived IL-25 is

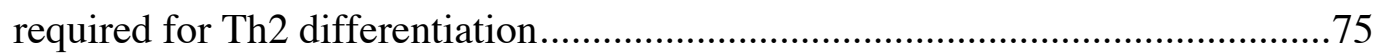

Figure 3. $12 \mathrm{Th} 2$ cells can be tracked over time in response to intradermal

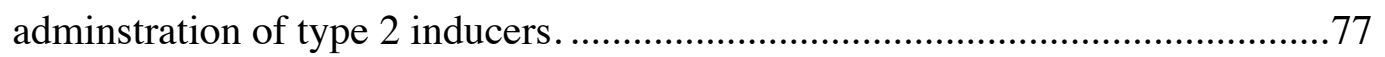

Figure 3. 13 Lack of IL-25 signalling has no effect on the expression of IL-4, IL-5 or

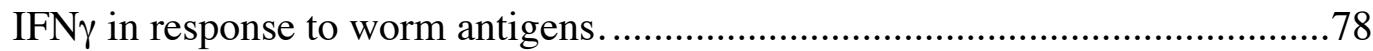

Figure 3. 14 IL-25 is not necessary for Th2 differentiation in vivo.......................... 79

Figure 4. 1 Il-25 is not required for development of Th2 cells in a Th2 effector or memory $\mathrm{T}$ cell mediastinal lymph node response setting .88

Figure 4. 2 Il-25 is not required for development of Th2 cells in a Th2 effector or memory $\mathrm{T}$ cell lung response setting.

Figure 4. 3 Loss of IL-25 signalling has no effect on type 2 cytokines investigated in the lungs following $N$. brasiliensis infection. ....................................................91

Figure 4. 4 Serum IgE titres are increased in mice deficient in IL-25 signalling . .......92

Figure 4. 5 IL-25 deficiency results in increased blood basophilia following secondary

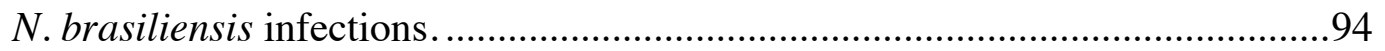

Figure 4. 6 A lack of IL-25 signalling resulted in increased blood eosinophilia following secondary $N$. brasiliensis infections.

Figure 4. 7 A lack of IL-25 signalling had little effect on eosinophilia in the lungs following secondary $N$. brasiliensis infections. .97

Figure 4. 8 IL-25 signalling is not required for production of lung mucous following infection with $N$. brasiliensis. .98

Figure 4. 9 Identification and characterisation of nuocytes in the lung, mediastinal and mesenteric lymph nodes following $N$. brasiliensis infection.

Figure 4. $10 \mathrm{IL}-25$ is not required for expansion of the nuocyte population in the mediastinal lymph node following $N$. brasiliensis infection. 101 
Figure 4. 11 IL-25 is not required for nuocytes expansion in the mediastinal lymph node following $N$. brasiliensis infection in the absence of IL-4. 102

Figure 4. 12 IL-25 is not required for nuocytes expansion in the lung following $N$. brasiliensis infection 103

Figure 4. 13 Even in the absence of IL-4, IL-25 is not required for nuocytes expansion in the lungs following $N$. brasiliensis infection. 104

Figure 4.14 Th2 cells rather than nuocytes are the predominant source of IL-4 in the mediastinal lymph node and lung during $N$. brasiliensis infection. 105

Figure 4. 15 Lung worm burdens are unaffected by loss of IL-25 signalling however a minor delay in expulsion from the small intestine is noted following both primary and secondary infections. 107

Figure 4. 16 IL-4 and IL-25 dual cytokine deficient animals have significantly impaired protective responses against secondary infections with $N$. brasiliensis.

Figure 4. 17 Mesenteric Th2 cells differentiate in the absence of IL-25 following infection with $N$. brasiliensis. 111

Figure 4. 18 Type 2 cytokines from the mesenteric lymph nodes of IL-25 deficient mice were similar to those of the IL- $25^{+/+}$controls following $N$. brasiliensis infection.

Figure 4. 19 Nuocyte frequency and numbers in the mesenteric lymph nodes of IL-25 deficient mice were similar to those of the controls following primary $N$. brasiliensis infection

Figure 4. 20 Loss of IL-25 resulted in delayed expulsion from the gastrointestinal tract following secondary infection with $H$. polygyrus. 114

Figure 5. 1 IL-4 expression is predominantly monoallelic, however the biallelic IL-4 population increases as the immune response increases. 130

Figure 5. 2 Biallelic cells have a higher expression of activation markers compared to monoallelic cells 132

Figure 5. 3 Biallelic cells express more IL-4 reporter proteins per cell than monoallelic cells. 132

Figure 5. 4 GFP Monoallelic compared to hCD2 monoallelic reporter positive CD4 T cells are more likely to secrete a second cytokine. 133 
Figure 5. 5 IL-4 expression is predominantly monoallelic whether the Th2 cells are located in the lung or the mediastinal lymph node. 135

Figure 5. 6 IL-4 heterozygous mice have a significantly impaired protective response against $N$. brasiliensis.

Figure 5. 7 IL-4 heterozygous mice have significantly impaired protective responses against infection with $H$. polygyrus.

Figure 5. 8 IL-4 heterozygous and IL-4 deficient mice have a significantly impaired B and Tfh cell compartment during infection with $N$. brasiliensis.

Figure 5. 9 IL-4 is required for a complete Th2 immune response against secondary infection by $H$. polygyrus. 143

Figure 5. 10 Biallelic IL-4 reporter positive CD4 T cells are more likely to express Tfh surface markers PD1 and CXCR5 than monoallelic IL-4 reporter positive CD4 T cells during $N$. brasiliensis infection 145

Figure 5. 11 IL-4 heterozygous and deficient mice have no difference in IL-4 reporter expression by Th2 cells during infection with $N$. brasiliensis.

Figure 5. 12 IL-4 wild type mice produce significantly more IL-4 secreting cells during $N$. brasiliensis infection when compared to IL-4 heterozygous mice. ...148

Figure 5. 13 The presence of IL-4 results in significantly elevated levels of transcription factors GATA3 and BCL6 in the lymph node but not the lung... 150

Figure 6. 1 A schematic describing the proposed roles for IL-25 during infection with gut dwelling helminthic stages of $N$. brasiliensis. .164

Figure 6. 2 The regulation of IL-4 through the monoallelic or biallelic expression of the $i l 4$ alleles is associated with functional subsets of Th2 cells. 166

Figure A. 1 The FACS plots are read from left to right and represent the hierarchical gating strategy used for analysing Th2 cells. 176

Figure A. 2 The FACS plots are read from left to right and represent the hierarchical gating strategy used for analysing the allelic expression of IL-4 by Th2 cells..176

Figure A. 3 The FACS plots are read from left to right and represent the hierarchical gating strategy used for analysing $\mathrm{T}$ follicular helper ( $\mathrm{Tfh}$ ) cells

Figure A. 4 The FACS plots are read from left to right and represent the hierarchical gating strategy used for analysing $\mathrm{IL}-4^{+}$eosinophils.

Figure A. 5 The FACS plots are read from left to right and represent the hierarchical gating strategy used for analysing basophils 
Figure A. 6 The FACS plots are read from left to right and represent the hierarchical gating strategy used for analysing nuocytes........ 


\section{List of Tables:}

Table 1 Surface characteristics of the lineage negative innate lymphoid cells (Adapted

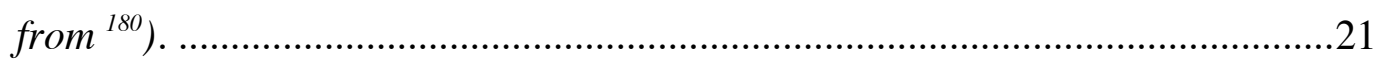

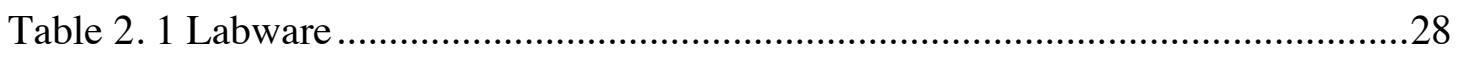

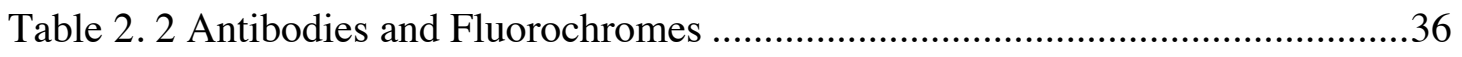

Table 2. 3 Antibody and Fluorochrome Detectors of the BD LSRII SORP (Becton

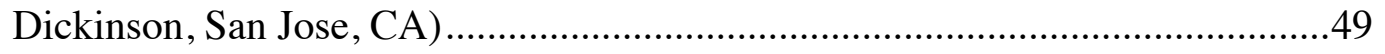

Table 2. 4 Laser Specifications for the BD LSRII SORP (Becton Dickinson, San Jose,

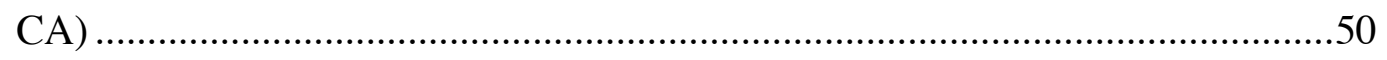




\section{List of Abbreviations:}
AAMac: Alternatively activated macrophage
Ag: Antigen
APC: $\quad$ Antigen presenting cell
BD: $\quad$ Below detection limit
BMDC: $\quad$ Bone marrow-derived dendritic cell
CD: $\quad$ Cluster of differentiation
DAPI: $\quad$ 4',6-diamidino-2-phenylindole
DC: $\quad$ Dendritic cell
DNA: Deoxyribonucleic acid
FACS: $\quad$ Fluorescence activated cell sorting
FBS: $\quad$ Foetal bovine serum
FCS: $\quad$ Forward scatter
Flt3L: $\quad$ Fms-like thyrosine kinase 3 ligand
GFP: $\quad$ Green fluorescent protein
GMCSF: Granulocyte macrophage colony stimulating factor
i.d.: $\quad$ intradermal
IFN: Interferon
i.g.: $\quad$ Intragastrically
IL: $\quad$ Interleukin
IMDM: Isove's Modified Dulbecco's Medium
i.p.: Intraperitoneal
KO: $\quad$ Knock out
L: $\quad$ Ligand
LPS: $\quad$ Lipopolysaccharides
mAb: $\quad$ Monoclonal antibody
MACS: $\quad$ Magnetic Cell Sorting
MdFI: Median fluorescence intensity
MedLN: Mediastinal lymph node
MesLN: $\quad$ Mesenteric lymph node
MHC: $\quad$ Major Histocompatibility Complex 
mRNA: $\quad$ Messenger ribonucleic acid

NBNT: $\quad$ non-B cell non- $\mathrm{T}$ cell

ND: $\quad$ Not determined

NKT cell: $\quad$ Natural killer T cell

OVA: Ovalbumin

PBS: $\quad$ Phosphate Buffered Saline

PCR: $\quad$ Polymerase Chain Reaction

PD-1: $\quad$ Programmed death receptor-1

PE: $\quad$ Phycoerythrin

PerCP: $\quad$ Peridinin Chlorophyll Protein

PI: Post inoculation

PTR: $\quad$ Prior to re-infection

R: Receptor

SAV: Streptavidin

s.c.: $\quad$ Subcutaneous

SEM: $\quad$ Standard error of the mean

SSC: $\quad$ Side Scatter

TCR: $\quad$ T cell receptor

Tg: $\quad$ Transgenic

Th cell: $\quad$ T helper cell

WT: Wild type 


Chapter One:

General Introduction 
An adaptive immune response evolves as a result of the directional leadership by a subset of $\mathrm{T}$ helper (Th) cells that have differentiated in response to cognate antigen. The signals initiating the mechanism of differentiation and the cellular and molecular requirements for the maintenance of effector and memory functions of these $\mathrm{T}$ helper subsets are key issues currently investigated by multiple groups around the world. Differentiated T cells are generally classified into four subsets; Th1, Th2, Th17 and inducible regulatory $\mathrm{T}$ (iTreg) cells with each subset classified as a result of their signature cytokines that drive specific effector responses. The T helper type 2 immune response is a poorly understood arm of the immune system, this lack of understanding results in difficulty in development of effective treatment strategies against allergies and asthma as well as parasitic helminths, highlighting a crucial need for further investigations to increase our chances of success.

The $\mathrm{T}$ helper type 2 immune response can be segregated into two arms; the adaptive CD4 or T helper (Th) 2 cell mediated responses and the innate or type 2 mediated responses. The segregation of the $\mathrm{T}$ helper type 2 immune response into $\mathrm{Th} 2$ cell responses and type 2 responses is useful as it allows one to compartmentalise and ask particularly pointed questions about fundamental mechanisms underlying this particular type of immunity. This thesis focus is on the adaptive CD4 Th2 cell response and asks pertinent questions with regards to the development and definition of this cell type. To honour the historically relevant definitions of helper cells, a Th2 cell herein is defined as a CD4 T lymphocyte that secretes IL-4, the signature cytokine of a $\mathrm{T}$ helper type 2 immune response. This thesis set out to investigate whether a novel T helper type 2 amplifying cytokine, IL-25, is involved in the differentiation and development of effector and memory Th2 cells and further goes on to investigate the classification of a Th2 cell by its ability to secrete IL-4 from independent alleles.

\section{1 Subsets of CD4 T helper cells and their involvement in immunity}

The key to differentiation and subsequent polarisation of $\mathrm{T}$ helper subsets is the cytokine milieu and co-stimulatory signals the naïve $\mathrm{T}$ cell experiences upon cognate antigen encounter. Naïve CD4 T cells exit the thymus and enter the secondary lymphoid organs, where they may be exposed to stimulation by antigen presented on 
the major histocompatibility complex (MHC) class II. This results in CD4 $\mathrm{T}$ cell activation and IL-2 production ${ }^{1}$. Subsequent autocrine and paracrine IL-2 signalling results in clonal expansion of these activated cells. Four distinct subsets of CD4 T cells differentiate upon appropriate activation signals (See Figure 1. 1); the inducible T regulatory cells (iTregs) ${ }^{2,3}$ and three effector cell subsets, Th1 ${ }^{4-6}$, Th2 ${ }^{6-8}$ and Th17 9-11. These four subsets comprise the commonly discussed arms of the helper $\mathrm{T}$ cell response. Additionally, two further T helper cell subsets have been described recently: the Th22 and the Th9 cell subsets. Th22 cells produce IL-22 and are theorised to have a predominant role in maintaining skin homeostasis ${ }^{12,13}$ while Th9 cells produce IL-9 and are theorised to provide a form of specialised protection during helminth infections ${ }^{14-16}$. Both inducible and natural Tregs are responsible for maintaining homeostasis and immune tolerance whereas the effector subsets induce protective immune responses against pathogens. IFN $\gamma$ producing Th1 cells mediate immunity to intracellular parasites, IL-4 producing Th2 cells mediate immunity to parasitic helminths while IL-17 producing Th17 cells mediate immunity to extracellular bacteria and fungi. Of note, the dysregulation of Th1 and Th17 effector arms results in autoimmunity while dysregulation of the Th2 arm results in allergies or asthma ${ }^{17}$. These helper subsets play vital roles through directing immune responses, roles that highlight a crucial need for understanding the signalling processes involved during subset differentiation and development with the goal of providing future targets for more effective drug design and therapeutic approaches. 
Extracellular bacteria

Fungi

Autoimmunity

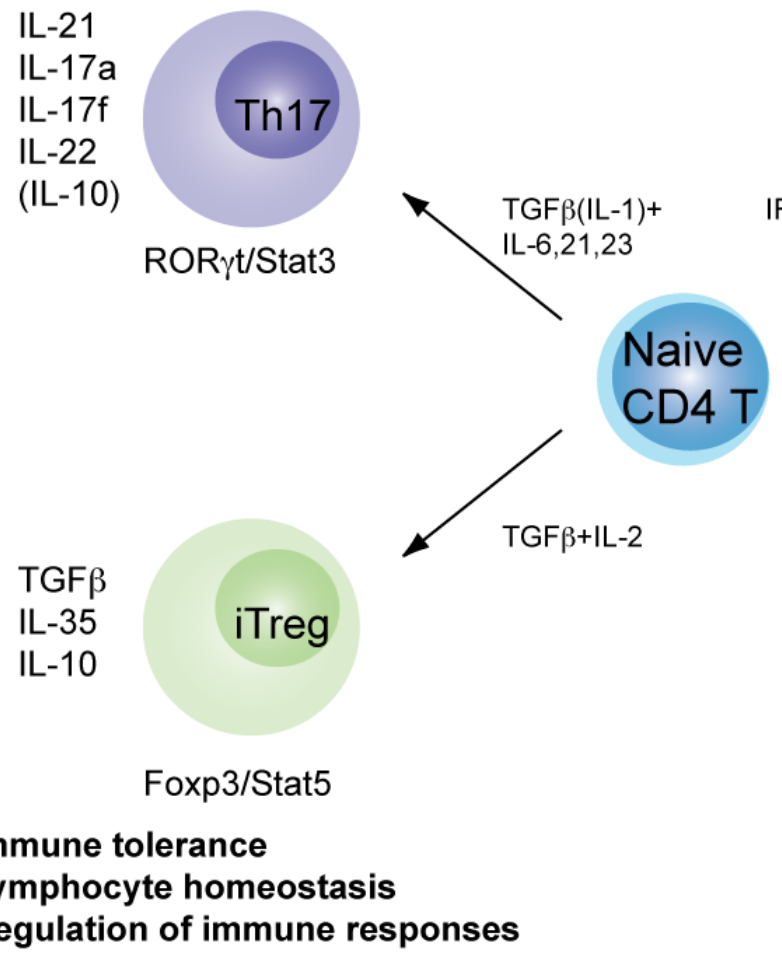

Intracellular pathogens

Autoimmunity

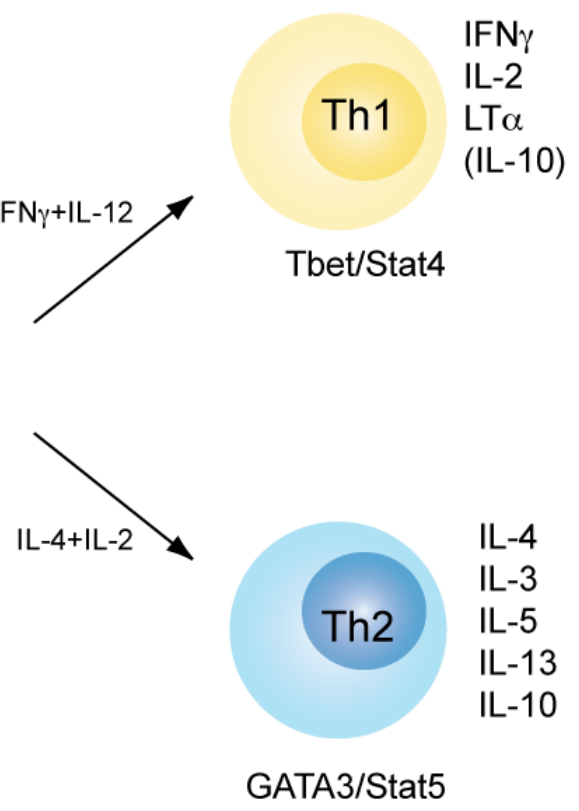

Extracellular pathogens

Allergy and asthma

Figure 1. 1 Diagram illustrating the four well characterised T helper subsets: their polarising cytokines, master regulator transcription factors, effector cytokines and immunological functions (Adapted from ${ }^{17}$ ). 


\section{2 Interleukin-4: The signature cytokine of a T helper 2 cell}

The suite of cytokines collectively described as Th2 cytokines include IL-2, IL-3, IL4, IL-5, IL-9, IL-10 and IL-13. IL-4, the signature cytokine of a Th2 response has been extensively investigated with respect to its signalling pathway and regulation of type 2 immunity. However, the factor critically required for differentiating naïve CD4 T cells into IL-4 producing cells remains unknown. IL-4 was initially described as a B cell growth factor ${ }^{18}$ and then as a B cell activation factor ${ }^{19}$. Subsequently it became know as the signature cytokine produced by Th2 cells that resulted in Th2 effector functions ${ }^{6}$. IL-4 was shown to be required for the maintenance and development of Th2 cells ${ }^{7,8,20}$ and the activation of $\mathrm{B}$ cells resulting in the increased expression of MHC class II and class switching to $\operatorname{IgG} 1$ and $\operatorname{IgE}{ }^{21,22}$. In addition to its potent effects on lymphocytes, IL-4 serves to activate innate effector cells including mast cells ${ }^{23-25}$, macrophages ${ }^{26}$ and eosinophils ${ }^{27}$ and also induces alteration in a variety of nonimmune cells such as mucosal epithelial cells ${ }^{28-30}$ and goblet cells ${ }^{31}$.

\section{3 Requirements for the differentiation of a Th2 cell}

Th2 differentiation from the naïve CD4 $\mathrm{T}$ cell requires TCR recognition of cognate antigen bound to MHC complex, co-stimulation and a polarising cytokine milieu: as summarised in Figure 1.2. The exact properties of antigens that drive Th2 responses remain largely unknown however certain in vitro conditions have been shown to skew a naïve CD4 $\mathrm{T}$ cell in the absence of polarising cytokine into a Th2 cell, these include low concentrations of available antigen ${ }^{32-34}$ and low avidity and affinity of the binding of TCR to antigen MHC complex ${ }^{35}$. Whether these findings depict the in vivo setting during allergic responses is the ongoing topic of debate ${ }^{36}$.

Dendritic cells are messenger cells of immunity, located in barrier sites searching for antigen, processing this antigen and migrating through to the lymph node where they can present it to naïve CD4 T cells ${ }^{37}$. Recent investigations however, have suggested basophils could be the critical antigen presenting cell (APC) type required for the differentiation of Th2 cells ${ }^{38-40}$. Conversely, other studies identify basophils as 
augmenting the Th2 response, with a clear requirement for DCs facilitating antigen presentation and Th2 cell differentiation ${ }^{41-44}$. Additionally, basophil depletion prior to and during infection with the robust Th2 inducing helminth $N$. brasiliensis resulted in no detrimental effects on Th2 differentiation ${ }^{45}$. Taken together, these data illustrate a limited role for basophils in Th2 differentiation, development or maintenance, however DCs are necessary and sufficient for the initiation of a Th2 response.

The stereotypical three part signals consisting of antigen TCR engagement, costimulation and polarising cytokine signals were initially identified for the induction of Th2 cells. Yet, the precise requirements for Th1 and even Th17 cells are understood with much greater clarity. Several studies have attempted to delineate the critical signals required from APCs to differentiate naïve CD4 T cells into Th2 cells. Notch signalling through jagged ligand ${ }^{46}$, OX40L ${ }^{47}$, ICOS ${ }^{48}$ and CD40 ${ }^{49}$ have all been shown to amplify Th2 differentiation. Additional co-stimulatory signals include the engagement of the CD4 molecule ${ }^{50}$ and CD28 molecule ${ }^{51}$ with the TCR complex assisting Th2 differentiation signals. Therefore, the activation status of the APC and hence the co-stimulation provided will have a large impact on the polarisation of the subset differentiated. 


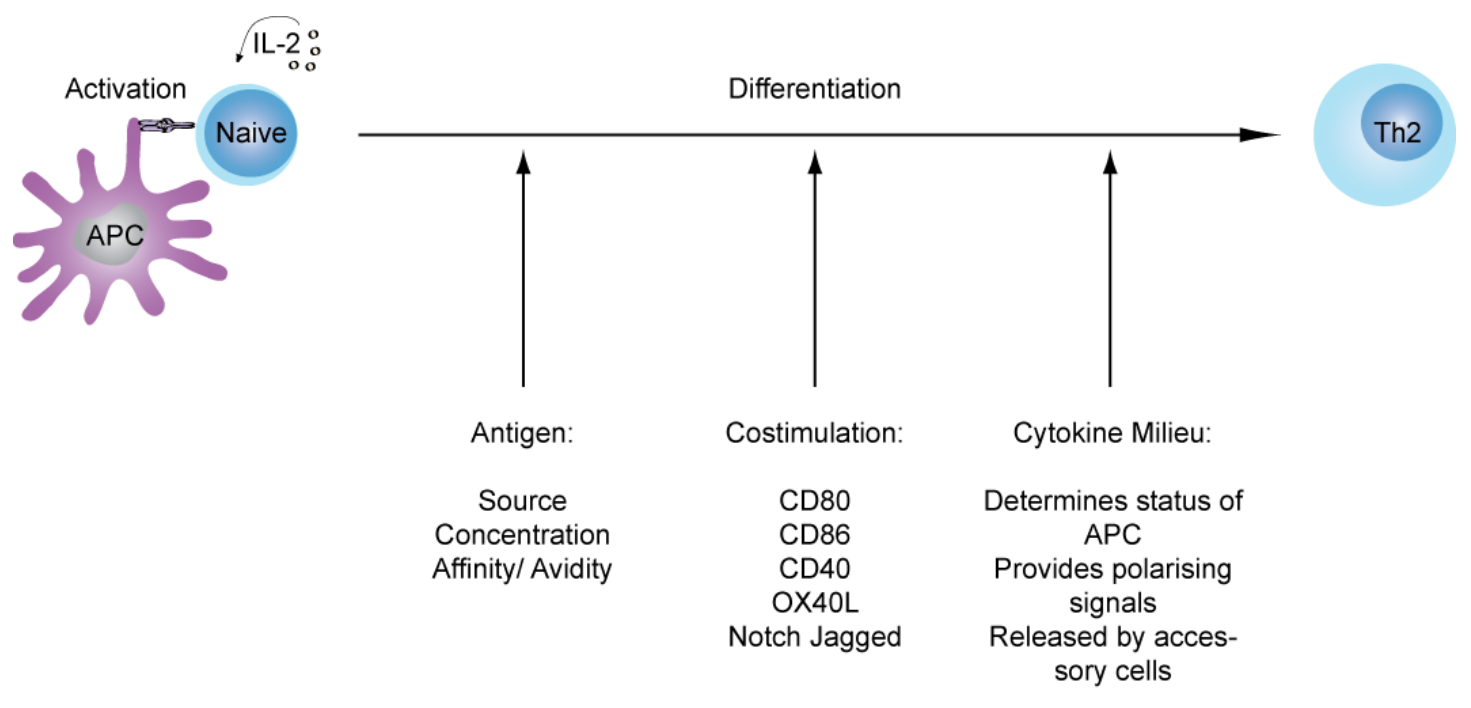

Figure 1. 2 Fundamental signals involved in Th2 differentiation. Often termed as signal 1, 2 and 3 they constitute the core requirements for differentiation of a naïve CD4 T cell into a Th2 cell: they comprise the engagement of the TCR by cognate antigen presented on the MHC class II complex, the provision of co-stimulation and the provision of a polarising cytokine milieu. The polarising cytokine milieu required for Th2 differentiation is unknown.

Initial investigations revealed a requirement for IL-4 along with IL-2 for the differentiation of Th2 cells ${ }^{7,8}$. The initial cell producing IL-4 remained a mystery. Multiple cell types have been shown to produce IL-4 including mast cells ${ }^{52,53}$, basophils ${ }^{54,55}$, Natural Killer T (NKT) cells ${ }^{56-59}$ and eosinophils ${ }^{27}$, along with more controversial reports of IL-4 production by $B$ cells ${ }^{60}$, lineage negative innate lymphoid type 2 cells (ILC2s) ${ }^{61}$ and naïve CD4 T cells ${ }^{62,63}$. It is unlikely these IL-4 producing naïve CD4 $\mathrm{T}$ cells are truly naïve as these $\mathrm{T}$ cells require an initial activation stimulus and TCR engagement in order to produce IL-4, and therefore could be perhaps better classified as early-differentiated Th2 cells. The production of IL-4 early during differentiation of naïve CD4 T cells fuels speculation this cytokine, acting in an autocrine manner, would reinforce Th2 development and its own production. Nonetheless, while IL-4 may have a role during the development and maintenance of a Th2 response ${ }^{20}$, it is not required for the differentiation of a naïve CD4 T cell to a Th2 cell ${ }^{63-67}$. The definition of the roles of IL-4 during development and maintenance of a Th2 response are not fully described, and the innate cytokine candidates, IL-33, TSLP and IL-25 have recently drawn substantial interest ${ }^{68,69}$. 


\section{4 Regulation of IL-4 expression in the Th2 cell}

The regulation of expression of IL-4 from a differentiated helper T cell occurs in a biphasic manner; initially IL-4 is expressed following engagement of the TCR by antigen on MHC II complex then expression is increased following the exposure to appropriate polarizing signals. Controversy remains as to what exactly comprises these polarizing signals that results in the differentiated Th2 cell. Initially a cytokine milieu comprising IL-2 and IL-4 was accepted as a prerequisite for the differentiation of Th2 cells ${ }^{8}$, however subsequent improvements in technology has lead to the realisation that Th2 cells differentiate in the absence of IL-4 ${ }^{65}$. However GATAbinding protein 3 (GATA3) is accepted the master regulator of the production of IL-4 and is required for the differentiation of Th2 cells ${ }^{70,71}$. This was shown through its overexpression in cell lines resulting in dominance of Th2 over Th1 and its absence resulting in the inability to express IL-4. Interestingly recent in vivo data raises queries as to whether this is the only mechanism of Th2 differentiation with novel data highlighting the production of IL-4 in germinal centres in cells that do not upregulate GATA3.

Therefore there are multiple pathways to the production of IL-4 from a T helper 2 cell. One of which is the engagement of IL-4 with its receptor resulting in the activation of the transcription factor signal transducer and activator of transcription 6 (STAT6) and upregulation of GATA3 ${ }^{72,73}$. Although as mentioned previously IL-4 signalling through STAT6 alone is not sufficient to induce Th2 differentiation, the binding of STAT5 to the Il4 locus is also required ${ }^{67,74}$. STAT5 binding occurs following ligation of cell surface receptors with their STAT5 signalling molecules including IL-2, IL-7 and TSLP ${ }^{75}$. The STAT5 enhanced expression of IL-4 is GATA3, STAT6 and IL4R $\alpha$ independent; STAT5 binds directly to the Il4 gene as a result of the ligation of IL-2 or through the binding of other STAT5 signalling molecules ${ }^{75,76}$. It is likely the ligand responsible for activation of STAT5 is dependent upon the spatial location of the CD4 T cell during Th2 cell development. For instance, STAT5 activating ligands found in the lymph node include activated $\mathrm{T}$ cells releasing IL-2 or stromal cells 
releasing IL-7, while another STAT5 ligand, the innate cytokine TSLP, is more prominently produced in non-lymphoid organs ${ }^{77-79}$.

Additional signals enhancing IL-4 production by Th2 cells consist of the innate cytokines IL-33 and IL-25; IL-33 enhances the effect of STAT5 signalling ${ }^{80}$ while IL25 maintains the expression of GATA3 ${ }^{81}$. Also, the Notch ligands were recently shown to upregulate GATA3 expression independently of STAT6 activation ${ }^{46,82,83}$. There are a multitude of intracellular signalling processes resulting in IL-4 production, some dependent upon IL-4 and others independent, all likely acting in conjunction with one another. Figure 1. 3 outlines the major intracellular activation pathways involved in Th2 cell differentiation. 


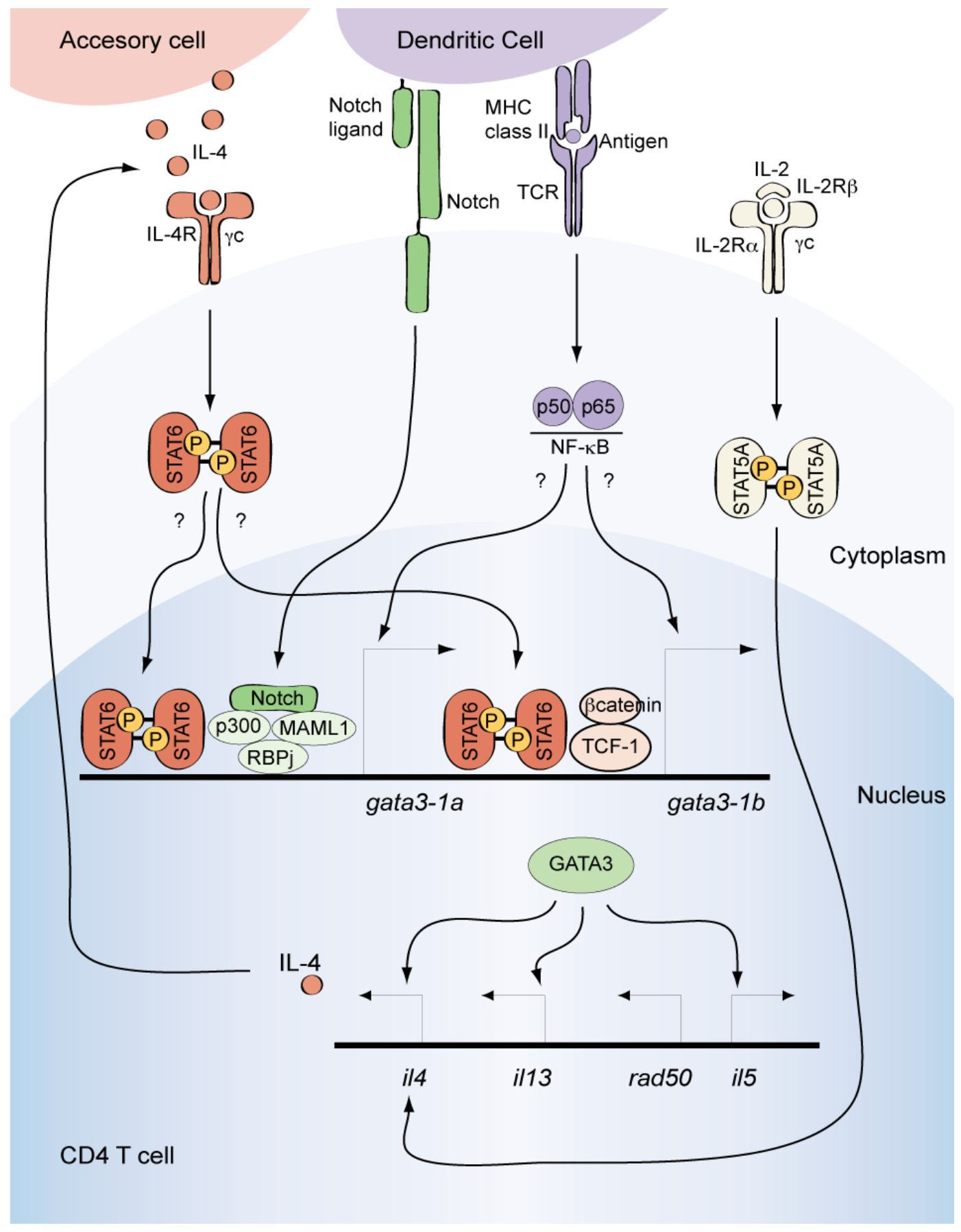

Figure 1. 3 Pathways of $T$ helper 2 cell differentiation, both IL-4 dependent and independent, require activation of GATA-binding protein 3. Early IL-4 production occurs by $T$ cell receptor (TCR) binding cognate antigen that is thought to signal through NFKB and trigger GATA3 expression. IL-4, from either an accessory cell or the CD4 T cell itself, upon binding the IL-4R triggers STAT6 phosphorylation and translocation to the nucleus where STAT6 binds unknown sites and upregulates GATA3 expression. IL-2 activates STAT5A and binds directly to the second intron of IL-4 resulting in the production of IL-4. Co-stimulation of notch by its ligand jagged results in translocation of intracellular notch to the nucleus forming a complex with recombination- signal-binding protein for immunoglobulin- $\kappa$ J region (RBPJ), Mastermind-like 1 (MAML1) and p300 that subsequently binds to and activates GATA3. GATA3 then modifies the IL-4/IL-13/IL-5 locus to enable access for further transcription factors and continued differentiation of the Th2 cell (Adapted from ${ }^{84}$ ). 


\section{5 Regulation of expression of IL-4 in a T follicular helper (Tfh) cell}

Germinal centres (GC) are located within antigen experienced B cell follicles in the secondary lymphoid tissues and are responsible for the production of memory B cells and antibody secreting plasma cells. GC formation requires CD4 T cell help as well as the expression of transcription factor B cell lymphoma 6 (BCL6) ${ }^{85-87}$. Spatially, the mature B cell follicle comprises a GC, made up of dark and light zones, with the dark zone adjacent to the $\mathrm{T}$ cell zone. The GC is capped by a follicular mantle of small non-antigen experienced re-circulating $B$ cells that is surrounded by non-circulating mature marginal zone B cells (see Figure 1.4). The formation of the GC allows antigen specific naïve B cells to undergo somatic hypermutation of the antibody variable V-region genes in the dark zone as well as class switch recombination of immunoglobulin heavy chain and affinity maturation of antigen binding capability in the light zone ${ }^{88-90}$. This GC reaction gives rise to memory B cells and plasma cells secreting high-affinity antibodies that are ultimately responsible for long term humoral immunity ${ }^{89,91}$. An alternative pathway external to the GC reaction (extrafollicular) gives rise to short-lived low-affinity antibody secreting plasma cells 92 .

The germinal centre dark zone consists of proliferating B cells known as centroblasts while the light zone contains non-proliferating B cells known as centrocytes, and these require survival signals from either the follicular dendritic cell or $\mathrm{T}$ cells to prevent their apoptosis ${ }^{93}$. The $\mathrm{T}$ cell zone serves as an area in which antigen-specific B cells can interact with cognate $\mathrm{T}$ cells that have been primed by dendritic cells specific for the same antigen. To greatly enhance the chance of an encounter between cognate antigen experienced B and T cells, antigen-bound B cells express both CCR7 and CXCR5 resulting in simultaneous attraction to both the T cell zone and the GC. CCR7 is the receptor for T cell zone cytokines CCL19 (ELC) and CCL21 (SLC), while CXCR5 is the receptor for B cell follicle cytokine CXCL13 (BLC/ BCA1) ${ }^{94-96}$. This combined expression results in the antigen-bound B cells hovering at the $\mathrm{T} / \mathrm{B}$ border 96-99. Antigen-activated CD4 T cells simultaneously up-regulate the expression of CXCR5 and down-regulate expression of CCR7 resulting in their subsequent attraction towards the $\mathrm{T} / \mathrm{B}$ border ${ }^{95,100}$. Upon conjugation, the $\mathrm{B}$ cell and $\mathrm{T}$ cells 
migrate into the $\mathrm{GC}$ resulting in $\mathrm{T}$ cell up-regulation of additional $\mathrm{Tfh}$ associated markers BCL6, ICOS, PD-1 and CXCR5 ${ }^{85,100-103}$. Once in the follicle, this specialised subset of CD4 $\mathrm{T}$ cells provide $\mathrm{B}$ cells with a variety of co-stimulatory interactions, most importantly co-stimulation by CD40L (CD154), as well as cytokines that result in B cell proliferation, maintenance, activation of maturation of the GC and long term humoral immunity ${ }^{104-108}$.

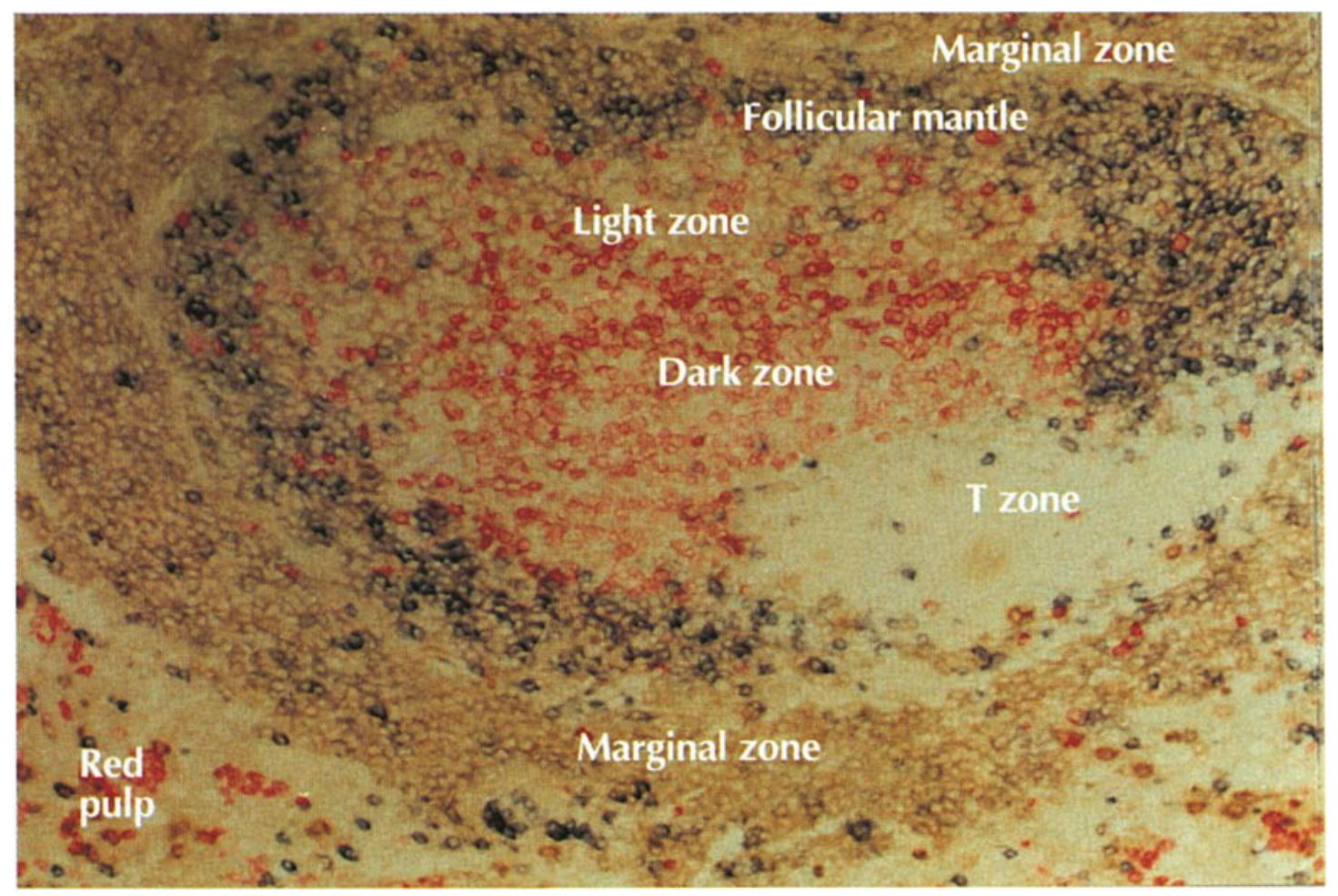

Figure 1. 4 Photomicrograph of a rat spleen section showing a well-developed germinal centre at the height of the germinal centre reaction. Most $B$ cells found in the spleen expressed IgM as noted by the gold staining, especially visible in the marginal zone of the mature non-circulating $B$ cells. The small circulating $B$ cells of the follicular mantle stained blue for IgD while there is a clear spatial distinction of the light zone, dark zone and T zone ${ }^{93}$.

Within the time span of the research contained in this thesis, the discovery was made that the majority of IL-4 producing CD4 T cells within the lymph node also express Tfh markers ${ }^{109-111}$. Th2 cells have been long thought of as the providers of T cell help to B cells through their production of IL-4 and the resultant proliferation and antibody class switch recombination that was initially noted in vitro ${ }^{18,112}$. With the discovery of Tfh cells, immunologists remain divided as to whether these cells should be dealt with as a separate subset of $\mathrm{T}$ helper cells or as the specialisation of a particular $\mathrm{T}$ helper 
subset. Tfh cells were initially characterised by their increased surface expression of $\mathrm{C}-\mathrm{X}-\mathrm{C}$ chemokine receptor type 5 (CXCR5) and the characteristic homing to the GCs ${ }^{105,106}$. The recent discovery of a master regulator of transcription, BCL6 ${ }^{101-103}$ along with distinct molecular and genetic expression patterns in the Tfh compared with Th2 cells ${ }^{113,114}$ further supports the hypothesis of a new helper subset.

The upregulation of CXCR5 results in Tfh cells attraction to the GC light zone where follicular stromal cells and follicular dendritic cells produce the CXCR5 ligand, CXCL13 (BLC, BCA1) ${ }^{94-96,115}$. The majority of CXCL13 in the B cell follicle is not expressed in the GC but rather expressed in the follicular mantle with far less expression in the light zone ${ }^{95}$. Both areas are rich in Tfh cells as shown by in situ imaging of B cell follicles ${ }^{116}$. A heterogeneous population exists within the $\mathrm{CXCR}^{+}$ CD4 $\mathrm{T}$ cells found in the B cell follicles, and GL-7 has recently been proposed to delineate GC specific populations ${ }^{117}$. Interestingly, these GL- $7^{+}$GC specific Tfh cells were shown to exclusively produce IL-4 ${ }^{117}$, and express the highest levels of BCL6 transcription factor as well as the Tfh signature cytokine, IL-21. Yusuf et al. propose this expression of IL-4 by GC Tfh cells is independent to 'conventional Th2 cell' differentiation, as the GATA3 levels in these CD4 T cells remains unaltered ${ }^{117}$. This concept of IL-4 production in specialised Tfh cells through a mechanism independent to 'conventional Th2 cell' IL-4 production (ie. through the master transcription factor GATA3) had been proposed earlier by Reinhardt et al., describing the production of IL-4 by Tfh cells as independent of STAT6 signalling or upregulation of GATA3 ${ }^{111}$. Subsequently, Vijayanand et al. extended this theory of IL-4 production in Tfh cells in a manner independent to that of IL-4 production in 'conventional Th2 cells', by describing the necessity of a conserved Il4 enhancer hypersensitivity site V for the production of IL-4 by T helper cells in the lymph node but not the periphery ${ }^{118}$. These recent discoveries illustrate how necessary it is to determine what really constitutes a Th2 cell and whether the production of IL-4 by CD4 T cells is the best characterisation of a Th2 cell. This is especially relevant with regard to the discovery that Il4 gene expression is subject to monoallelic regulation in vitro ${ }^{119-121}$, a form of regulation which could potentially offer an additional level of control in distinguishing functional subsets of IL-4 producing CD4 T cells. 


\section{6 Regulation of class-switch recombination of B cells to IgE by IL-4 producing CD4 T cells}

Class-switch recombination occurs in the germinal centres of lymphoid tissues with $\mathrm{T}$ cell help and results in naïve B cells, which express only $\operatorname{IgD}$ and $\operatorname{IgM}$, altering their heavy chain expression to $\operatorname{IgG}$, IgA or $\operatorname{IgE}{ }^{122}$. Serum concentrations of immunoglobulins vary, with IgM being prominent early in the immune response, followed by $\operatorname{IgG}$. IgE is found at low concentrations in the serum due to its markedly short half-life ${ }^{123}$. The majority of IgE antibodies usually have low binding affinity to antigen, with the exception of certain autoimmune responses and during infections with helminths ${ }^{124}$. The binding of IgE by Fce receptors on basophils and mast cells stabilises IgE and prolongs its half-life ${ }^{125}$. Upon re-encounter with antigen, crosslinking of bound IgE molecules causes activation and degranulation of the effector cell. The low affinity IgE competes with the sparsely numbered high affinity $\operatorname{IgE}$ for Fce receptors thereby preventing cross-linking and activation of the carrier cell ${ }^{126}$. The generation of high affinity IgE antibodies have been shown to class-switch from $\operatorname{IgM}$ to $\operatorname{IgG} 1$ and then on to $\operatorname{IgE}{ }^{126,127}$ however the exact mechanism of $\operatorname{IgE}$ class switch, memory cell and plasma cell formation remains highly debated due to the difficult nature of detection of the immunoglobulin ${ }^{128,129}$. Animals lacking IL-4 or STAT6 have decreased IgG1 and no IgE class switch recombination 111,130,131, indicating different polarising signals are required for IgG1 and IgE, with IL-4 critical for the production of $\mathrm{IgE}^{132}$. IL-21 receptor signalling is required for regulation of $\operatorname{IgE}$ production, with investigations demonstrating excessive IgE production in the absence of IL-21 receptor ${ }^{133}$. The mechanism resulting in increased IgE production has yet to be fully elucidated. Both the production of IL-4 and the provision of help by the Tfh cell subset are required for the induction of the hallmark Th2 pathology of B cell class switching to $\operatorname{IgE}{ }^{111,132}$. It is presumable that there are multiple as of yet unknown regulatory processes involved in IgE production, especially considering increased titres of high affinity antibodies would result in increased likelihood of anaphylaxis and potential mortality. Of interest to this thesis is the regulation of the production of IL-4 by the CD4 T cell that is responsible for the classical Th2 hallmark response of class-switching to IgE. 
The definition of a Th2 cell is rather broad and in many respects poorly defined. Originally Th2 cells were described as the CD4 T cell subset that produced IL-4 a B cell growth, proliferation and class-switch signalling molecule ${ }^{6}$. However subsequently CD4 $\mathrm{T}$ cells have been labelled as Th2 cells when making IL-4 mRNA ${ }^{134}$ or when exhibiting up-regulation of GATA $3{ }^{135}$ even though this does not mean that these cells will produce IL-4 protein. In fact, debate remains as to whether CD4 T cells producing IL-4 protein and expressing Tfh markers should be labelled Th2 cells 109-111,113. Perhaps the issue is the broad term Th2 cell; perhaps the Th2 cell subset should be thought of in terms of multiple specialised subsets based on their effector functions. For instance Th2 cells responsible for B cell class switch to IgE, Th2 cells that are effector cell mediating eosinophilia or mucous hyperplasia and Th2 cells that are responsible for the induction of memory against parasitic helminths and allergens. This concept is summarised in Figure 1.5 below and will be further investigated when determining whether the genomic signature of the Th2 cell can be used to better characterise the Th2 cell subset. 


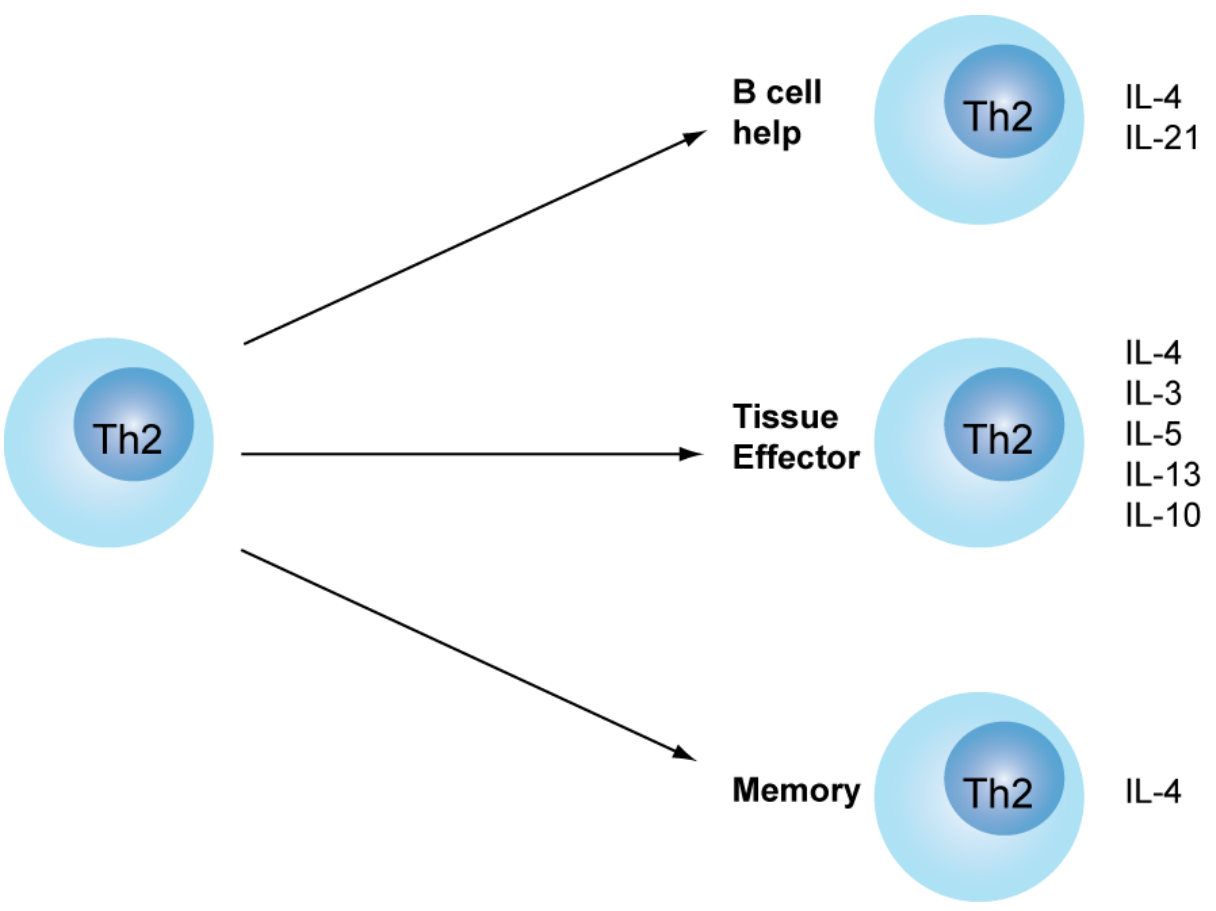

Figure 1. 5 The differentiated Th2 cell population comprises multiple functionally distinct subsets. Th2 cell subsets specialise to provide help for B cell class switch recombination and germinal centre formation, for localisation to tissue sites with specific cytokine repertoire for effector functions, for the establishment of central and effector memory responses.

\section{7 Interleukin-25: A Th2 associated innate cytokine}

Interleukin-25 (IL-17E) is 1 of 6 cytokines that make up the IL-17 family, however unlike the other members of its family, IL-25 recruits eosinophils rather than neutrophils and enhances Th2 associated responses such as mucous hyperplasia and class switching to IgE rather than autoimmune responses ${ }^{136-139}$. IL-25 signals through IL-17RB in conjunction with IL-17RA, initiating NF- $\kappa B$ signalling via MAP kinase activation and the adaptor molecule TRAF6 ${ }^{139-142}$. Activation of the naïve CD4 T cell by IL-25 is associated with the early expression of the master Th2 transcription factor GATA3, even in the absence of signalling by IL-4 ${ }^{81}$ inferring signalling by IL-25 could potentially act as an early polarisation factor for the differentiation of naïve CD4 T cells into Th2 cells. 
Initially IL-25 proved a difficult cytokine to detect, and was originally thought to be expressed exclusively by Th2 cells of a highly polarised phenotype ${ }^{138}$. Subsequently other cell types including IgE cross-linked bone marrow derived mast cells ${ }^{143}$, allergen stimulated epithelial cells ${ }^{81}$ and alveolar macrophages ${ }^{144}$ along with a small population of caecum resident CD4 and CD8 T cells in naïve animals have been shown to produce IL-25 constitutively ${ }^{145}$. Cells that respond to IL-25 include macrophages, dendritic cells ${ }^{146}$, memory Th2 cells ${ }^{147}$, a subset of NKT cells ${ }^{148,149}$ and innate lymphoid type 2 (ILC2) cells ${ }^{61,150-152}$. As resources improve a clearer understanding of exactly which cells produce and respond to IL-25 in physiologically relevant situations will develop. Original studies showed that administration of rIL-25 resulted in enhanced Th2 associated phenotypes in vitro and in vivo, and these data lead to IL-25 being described as "an initiation factor as well as an amplification factor for Th2 responses" ${ }^{17}$. Current speculation places the production of IL-25 by initial antigen barrier cells, such as the epithelium, altering the phenotype of the APC, that potentially also secretes IL-25, resulting in the differentiation of the Th2 cell. IL-25 is also proposed to have a role in maintenance of effector and memory Th2 cells, supplied either by the Th2 cell itself or from an accessory cell type, that results in expansion of the Th2 population and maintenance of IL-4 secretion (See graphic summary in Figure 1.6). 


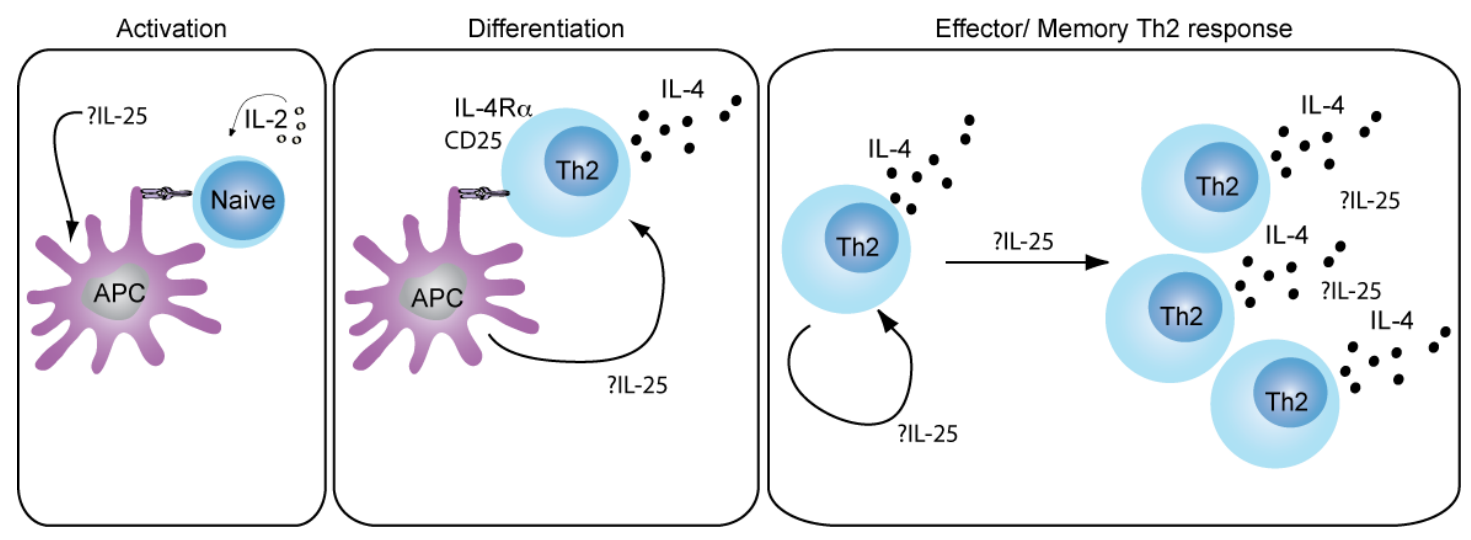

Figure 1. 6 Potential roles for IL-25 during differentiation and development of a Th2 immune response. The innate cytokine IL-25 is associated with Th2 pathologies, yet how signalling by IL-25 causes these Th2 responses remain unknown. Multiple opportunities for a role for IL-25 during the differentiation and development of the Th2 response exist and these include: Altering the phenotype and hence the signal given by the antigen presenting cell (APC) to the naïve CD4 T cell; acting as a polarising cytokine during differentiation of the Th2 cell; acting in an autocrine/ paracrine feedback supporting Th2 maintenance and/or proliferation and production of IL-25 by Th2 cells for activation of effector cells involved in Th2 immunity.

\section{8 IL-4 mediated immune responses to parasitic helminths}

Immunity against parasitic helminths is mediated by the Th2 subset of helper $\mathrm{T}$ cells 153-155. Nippostrongylus brasiliensis is a nematode whose lifecycle in mice closely resembles hookworm infections in humans ${ }^{156}$. These parasites enter their host through the skin as L3 infective stage larvae, and are thought to migrate through the blood vessels until they end up in the narrow capillaries of the lung where they break out into the alveolar spaces resulting in both tissue damage and, as they moult to L4 stage larvae, the deposition of antigen. The lung phase is associated with a Th2 mediated allergic airways inflammatory response that becomes chronic and ultimately resembles emphysematous disease ${ }^{157-160}$. The larvae are detectable in the lungs within 12 hours of infection and reach their highest numbers within 48 hours, with the majority having passed through by 72 hours ${ }^{161}$. The L4 larvae make use of the mucociliary ladder, moving up the bronchiole to be swallowed and ultimately ending up in the small intestine. Following a final moult in the small intestine, the sexually 
mature L5 larvae copulate and complete their lifecycle by producing eggs that are expelled into the environment enabling the initiation of a new infection cycle ${ }^{162}$. An immunocompetent mouse will expel L5 N. brasiliensis gut dwelling larvae within 810 days; this expulsion is dependent upon CD4 T cells and signalling by innate cell derived IL-4 and IL-13 via the STAT6 signalling pathway, that results in a 'weep and sweep' expulsion mechanism. This 'weep and sweep' expulsion mechanism comprises an increase in luminal fluids, mucous production, anti-parasitic molecules such as Relm- $\beta$ and an increase in peristaltic movements all ultimately leading to a hostile environment for the parasite and its expulsion from the gastrointestinal tract 155,163-166.

During the expulsion of $N$. brasiliensis from the small intestine, IL-4 can compensate for the loss of IL-5 and IL-9 ${ }^{167}$, however for timely expulsion IL-13 is critical ${ }^{168}$. The expulsion from the small intestine is characterised by increased mucous production, luminal fluids and smooth muscle contractility, and these in combination result in a hostile environment and the associated 'weep and sweep' expulsion mechanism ${ }^{169,170}$. Key contributors to this 'weep and sweep' mechanism include signalling by IL-4/IL13 through goblet cells that result in $\operatorname{Re} \operatorname{lm} \beta$ production ${ }^{171,172}$ as well as Muc5ac production ${ }^{170}$. Both molecules have been shown to directly inhibit parasite viability. IL-4/IL-13 activates macrophages that in turn result in smooth muscle cell activation ${ }^{173}$. Conversely, IL-4/IL-13 signalling may activate smooth muscle cells directly ${ }^{174}$, resulting in hypercontractility and increased mucous secretion, all factors contributing to parasite expulsion as summarised in Figure 1. 7. The source of this IL-4/IL-13 has recently been described to be a novel subset of lineage negative innate lymphoid cells 61,150-152,175

This novel subset of lineage negative innate lymphoid cells associated with Th2 responses has been described under a variety of names including natural helper cells (NHCs) ${ }^{152}$, multi-potent progenitor type $2\left(\mathrm{MMP}^{\mathrm{type} 2}\right)$ cells ${ }^{151}$, nuocytes ${ }^{61}$ and innate type 2 helper (Ih2) cells ${ }^{150}$. It remains in debate as to whether these cells should be described as independent cell types or under a composite term such as innate lymphoid type 2 (ILC2) cell ${ }^{176,177}$. The first in depth description of these innate lymphoid cells was that of the NHCs, which were described as absent from fat 
associated lymphoid clusters in common- $\gamma$ chain deficient and IL-7 deficient mice. Additionally, they were shown to be responsible for the production of type 2 cytokines involved in mucous production associated with $N$. brasiliensis infection ${ }^{152}$. These NHCs were lineage marker negative, Sca- $1^{+}, \mathrm{c}-\mathrm{Kit}^{+}, \mathrm{IL}-7 \mathrm{R} \alpha^{+}, \mathrm{CD} 25$ (IL-2R) ${ }^{+}$ and resembled lymphocytes in both size and granularity. NHCs showed prolonged survival or proliferation in vitro in response to the addition of $\mathrm{rSCF}$ (the c-Kit ligand), rIL-7 and rIL-2. What was remarkable was the maintenance of the NHC phenotype upon cytokine and polyclonal activation stimuli, illustrative of a terminal state of differentiation for the NHC population. The expression of IL-13 and IL-5 by NH cells occurred when stimulated with rIL-25 in combination with rIL-2 or rIL-33 alone and lead to the proposal that these cells could be the innate arm of a type 2 immune response.

IL-25 deficient animals were shown to have a delayed induction of Th2 responses, with delayed IgE production, delayed IL-13 and IL-5 production and delayed $N$. brasiliensis expulsion ${ }^{175}$. This has lead to the question of whether IL-25 is an important factor required for initiation events during Th2 differentiation or effector cell function. Subsequently, lineage negative non-T/non-B cell c-Kit ${ }^{+}, \mathrm{Fc \varepsilon R} 1 \alpha^{-}$cells (nuocytes) were adoptively transferred into IL-25R deficient animals and shown to mediate $N$. brasiliensis expulsion in an IL-13 dependent manner ${ }^{61}$. Nuocytes were described as the largest cell population producing IL-13 in the mesenteric lymph nodes at day 5 following $N$. brasiliensis primary infection. Nuocytes were expanded in the presence of rIL-25 and rIL-33 and were detectable at low frequencies in naïve animals in the spleen, mesenteric lymph nodes and bone marrow. Published simultaneously was a description of MMP ${ }^{\mathrm{type} 2}$ cells, which had similar characteristics to NHC and nuocytes, except that upon additional stimulation MMP ${ }^{\mathrm{type} 2}$ cells had the ability to differentiate into monocyte/macrophage or granulocytes and the ability to promote Th2 cell differentiation ${ }^{151}$. These data strongly suggest signalling by the cytokine IL-25 could potentially play a key role in the differentiation and/ or maintenance of Th2 cells. The final name given to these lineage negative cells was that of the Ih 2 cells, a deference to the traditional Th1/Th2 subset names, and this report illustrated the wide-spread, albeit low frequency, of these cells in most tissues at steady state ${ }^{150}$. A summary of surface characteristics of NHCs, nuocytes, MMP ${ }^{\text {type } 2}$ 
cells and Ih2s can be found in Table 1. It is thought these innate cells represent a key effector cell crucial in mediating effective Th2 immune responses.

\begin{tabular}{|c|c|c|c|c|}
\hline \multicolumn{5}{|c|}{$\begin{array}{l}\text { Table } 1 \text { Surface characteristics of the lineage negative } \\
\text { innate lymphoid cells (Adapted from }{ }^{178} \text { ). }\end{array}$} \\
\hline & Nuocyte & Ih2 & $\mathrm{NH}$ cell & $\mathrm{MMP}^{\mathrm{type} 2}$ \\
\hline c-Kit & $+/-$ & +1 & + & + \\
\hline CD45 & + & + & + & + \\
\hline IL-7R $\alpha$ & lo & $?$ & + & $-/ 10$ \\
\hline Sca-1 & + & - & + & + \\
\hline Thy-1 & + & + & + & $?$ \\
\hline CD34 & - & $?$ & - & $-/ 10$ \\
\hline CD25 & - & + & + & $?$ \\
\hline CD44 & + & + & + & $?$ \\
\hline FceR1 $\alpha$ & - & $?$ & - & - \\
\hline
\end{tabular}




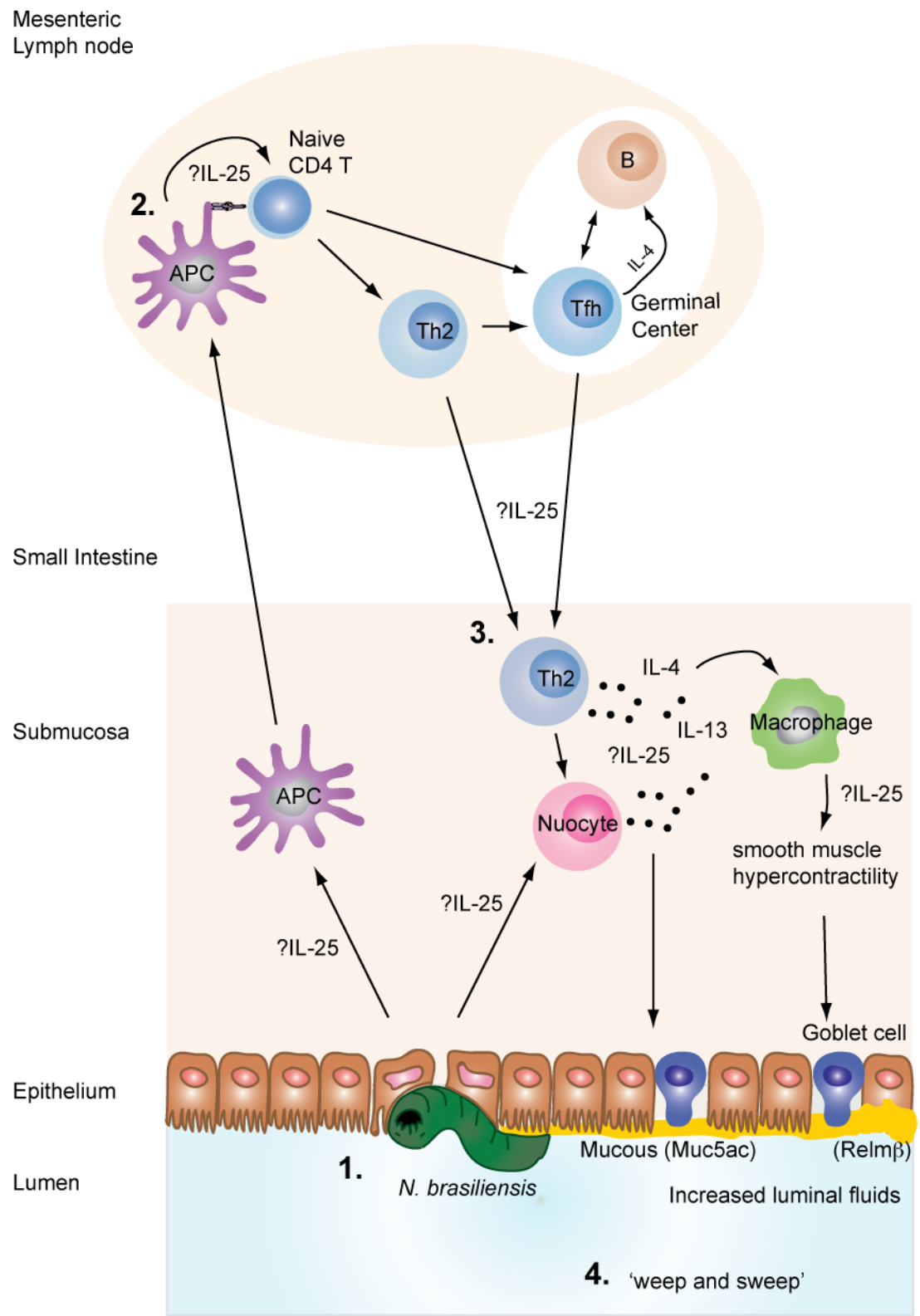

Figure 1. 7 Expulsion of primary Nippostrongylus brasiliensis is dependent upon Th2 mediated immunity. L5 N. brasiliensis larvae burrow into the small intestine releasing antigen, driving innate responses that could involve signalling by IL-25 from the epithelial layer. Antigen presenting cells migrate to the lymph node differentiating naïve CD4 T cells into Th2 cells, which migrate back to the tissues and assist timely expulsion. IL-13 produced by a non-T/ non-B cell, thought to be the nuocyte, activates macrophages, epithelial cell and smooth muscle cells resulting in goblet cell hyperplasia, Muc5ac and Relm $\beta$ production, smooth muscle hypercontractility and increased luminal fluids all contributing to the expulsion of the parasite (Adapted from ${ }^{179}$ ) 
Memory responses to $N$. brasiliensis infections are slightly different to those observed during primary infections. Unlike primary responses that critically require IL-13, the generation of protective immunity has a crucial requirement for CD4 T cells and signalling by IL-4 ${ }^{161}$. The most distinguishing factors of the generation of memory include the rapid halting of the parasite infection cycle evidenced by significantly lower worms travelling through the lungs and even fewer reaching the small intestine. This protective immunity is observed within 24 hours in the lungs. The worm burdens in this organ during a secondary infection are approximately 10 fold lower than that detectable during primary infection ${ }^{161}$. The depletion of macrophages with clodronate liposomes, however, results in significantly decreased protective response, as noted by increased worm numbers, illustrating a role for these cells in early protective immunity (Personal communication, Elizabeth Forbes. MIMR, New Zealand). The exact mechanisms of protective responses driven by the CD4 $\mathrm{T}$ cell and IL-4 signalling remains to be defined; it is plausible a number of effector cells including macrophages, neutrophils and eosinophils work in concert to halt the invasive parasite migration at the lung site and thereby prevent completion of the parasite lifecycle and transmission to a new host (See summary Figure 1. 8). This thesis investigates the interplay between IL-4 signalling and protective immune responses to parasitic helminths attempting to better understand how this immunity is generated, with a particular interest in the innate cytokine IL-25. Specifically, whether this Th2 associated cytokine may lie upstream of these IL-4 signalling pathways, and mediate the lung protective phenotype following challenge infections with $N$. brasiliensis. 


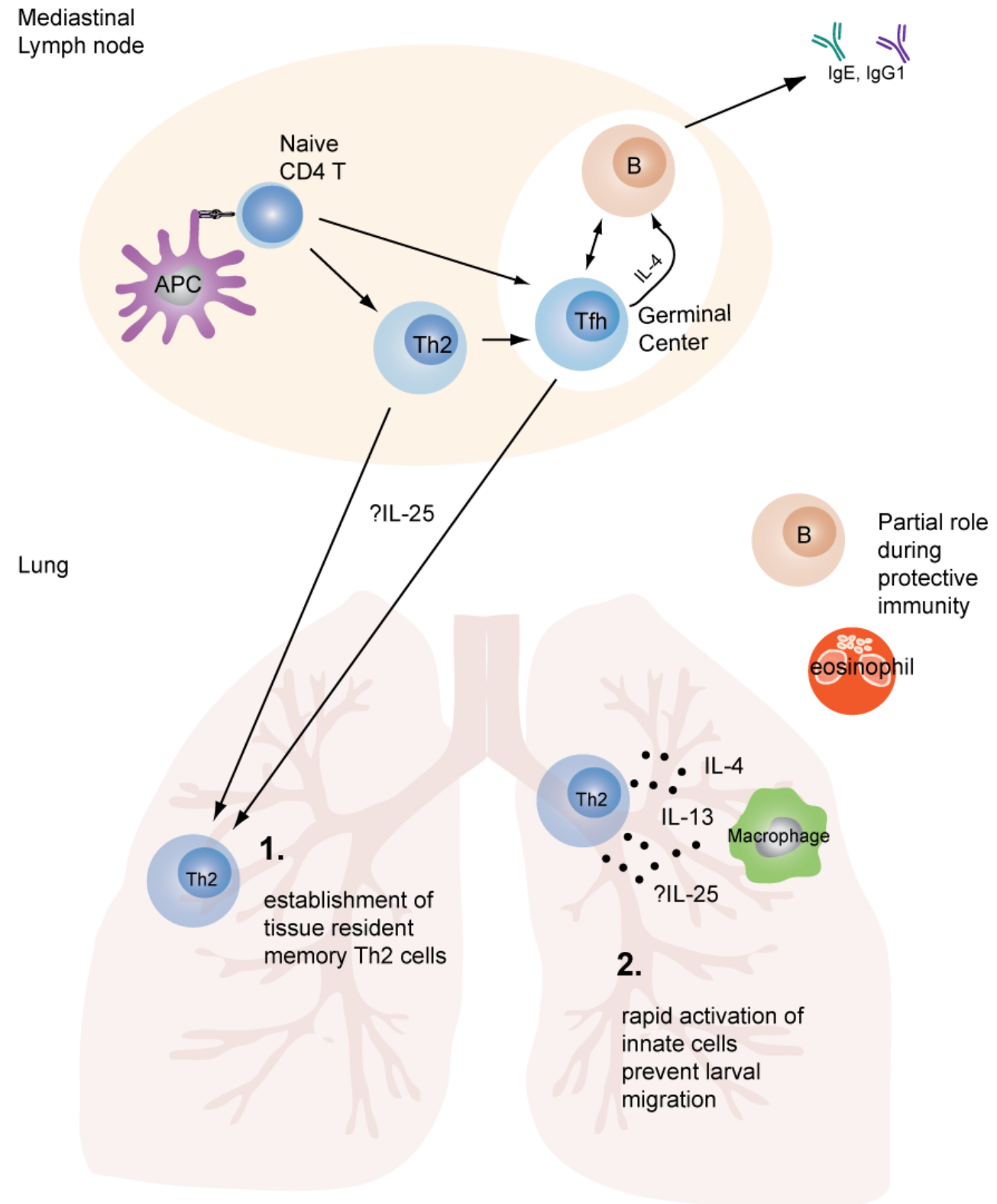

Figure 1. 8 Memory Th2 responses to Nippostrongylus brasiliensis are generated in the lung. Memory Th2 cells are established in the lung following the primary response, these Th2 cells along with IL-4/IL-13 activated macrophages, mediate immunity to challenge infections. Whether the Th2 associated cytokine IL-25 is required for establishment of the memory Th2 cells in the lung or for their subsequent effector function was examined in the studies within this thesis. 
Infectious L3 stage Heligmosomoides polygyrus larvae are ingested, pass through the stomach and enter the small intestine, where they bury into the sub-mucosa and undergo two moults before entering the lumen as L5 larvae. These L5 larvae exit the sub-mucosa within 8-10 days of infection and take up residence in the lumen, where they copulate and release eggs and are thought to survive by feeding on the epithelial layer of the villi ${ }^{180}$. Immunocompetent mice take months to expel mature $H$. polygyrus larvae, illustrative of the strong immunomodulatory capacity of the parasite on the host immune response ${ }^{181}$. A rapid and effective Th2 memory response can be induced if the primary infection is cleared artificially through administration of an anti-helminthic drug. Following drug treatment, protective immunity enables the host to expel the challenge infection within 2-3 weeks ${ }^{182}$. Blocking IL-4 cytokine results in a significant reduction in protective immunity, however, blocking the IL-4 receptor results in complete loss of protective immunity ${ }^{182}$. These data illustrate an important role for IL-4, the signature Th2 cytokine, but also highlights a probable role for IL-13 given the IL-4R requirements for IL-13 signalling. Investigations into mechanisms of protection ruled out, through IL-5 depletion, the necessity for eosinophils ${ }^{182}$, but highlighted a role for alternatively activated macrophages (AAMacs) and memory IL4 producing Th 2 cells ${ }^{183}$. Immunity to $H$. polygyrus occurs in two stages, according to the niche the parasite occupies, whilst in the sub-mucosa a granulomatous lesion surrounds the parasite where IL-4 mediated AAMacs, Th2 cells ${ }^{183}$, antibody producing B cells ${ }^{184,185}$ and granulocytes ${ }^{186}$ attack the parasite. The parasites that escape this phase and enters the lumen are faced with further IL-4 mediated responses. These include antibody reduced worm fecundity and a 'weep and sweep' mechanism that involves changes in epithelial cell functions, goblet cell secretion of mucous and $\operatorname{Re} \operatorname{lm} \beta$ and increased smooth muscle hypercontractility ${ }^{29,172,187}$. To date, no research has been published with regard to the role of IL-25 signalling in the generation of protective responses against challenge infections with $H$. polygyrus. As immunity to $H$. polygyrus requires both memory Th2 cells, macrophages and a 'weep and sweep' mechanism it is quite plausible IL-25 may provide a critical innate signal for inducing or maintaining this immunity (See summary Figure 1.9). 


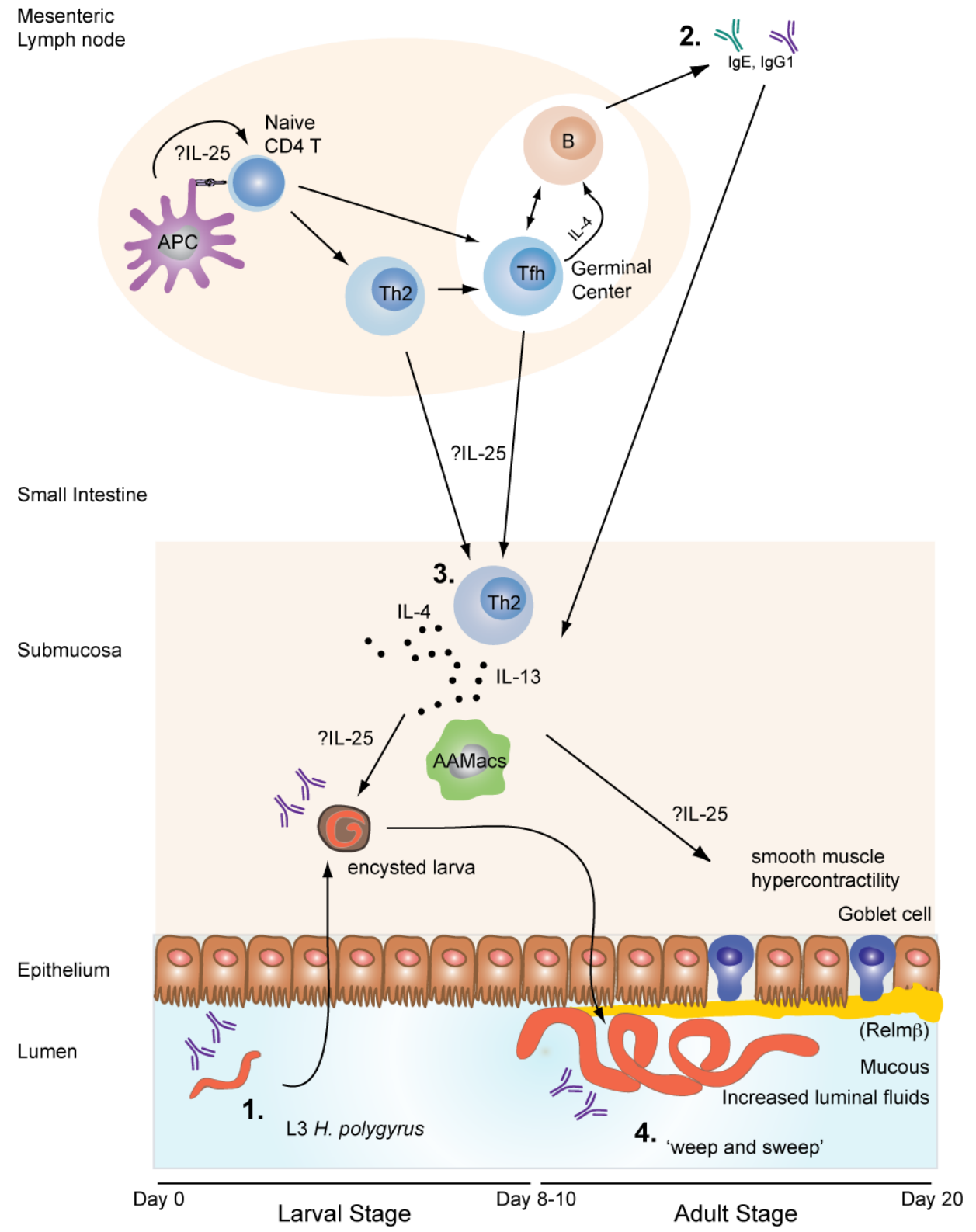

Figure 1. 9 Protective immunity against challenge infections by Heligmosomoides polygyrus is dependent upon Th2 cells and alternatively activated macrophages (AAMacs). Infective L3 stage larvae are ingested and travel to the small intestine where they enter the submucosa, encyst and undergo 2 moults. They emerge between 8-10 days later as sexually mature L5 adult larvae. Memory Th2 cells mediate an IL-4/ IL-13 specific AAMacs, granulocyte and antibody specific immune reponse against the encysted larvae stage and a 'weep and sweep' mechanism results in increased luminal fluids, mucous and smooth muscle hypercontractility against the adult L5 stage. It is unknown if signalling by the innate cytokine IL-25 is involved in mediating immunity to this parasite (Adapted from ${ }^{188}$ ).

\section{9 Cytokine reporter mice}

The best-known method of Th2 cell characterisation is by monitoring the production of the signature cytokine, IL-4. However monitoring IL-4 is not a simple undertaking, 
the cytokine has a very short half-life and is produced at low quantities and therefore usually requires manipulation ex vivo for detection by traditional methods (e.g. enzyme-linked immunosorbent assay (ELISA), intracellular flow cytometry and even the more modern enzyme-linked immunosorbent spot (ELISPOT) assays). With the advent of mouse reporter technology detection of IL-4 producing Th2 cells can now be done directly ex vivo without additional manipulation. Cytokine reporter mice generally make use of a naturally fluorescent or easily detectable and stable reporter protein that is expressed under the control of the promoter of the gene of interest. This reporter protein will therefore be produced either in concert or instead of the gene of interest and serves as a valuable tool for reporting the local microenvironment.

The development of reporter technology rapidly expanded our understanding of in vivo development and distribution of IL-4 producing cells with the generation of IL-4 reporter strains including the 4 get mouse ${ }^{134}$, the membrane bound IL-4 (mIL-4) mouse ${ }^{189}$, the KN2 mouse ${ }^{190}$, the hCD2 mouse ${ }^{121}$, and the G4 mouse ${ }^{119}$. These mice were used to map IL-4 producing cells in various immune contexts but of interest to this thesis is the use of these strains whilst mapping the generation of Th2 cells. The 4get mouse has an internal ribosome entry site-green fluorescent protein (IRES-GFP) cassette inserted into the 3' untranslated region of the Il4 locus thereby enabling the expression of both IL-4 protein and GFP reporter protein. This mouse continues to report the expression of IL-4 reporter protein even when the Th2 stimulus has been removed, unlike the mIL-4, hCD2, KN2 and G4 reporter mice strains that are all knock in mice (i.e. there is a targeted insertion of a reporter protein that is now expressed in the place of the wild type protein). Of interest is the difference in expression patterns of IL-4 reporter proteins from CD4 $\mathrm{T}$ cells of the knock in IL-4 reporter strains versus the 4get bicistronic reporter strain, the knock in strains only expressed IL-4 reporter protein during Th2 stimulation whilst the 4get strain continued to express IL-4 reporter protein even after stimulation was removed. This discrepancy lead to the discovery that the bicistronic reporter system of the 4 get mouse was reporting IL-4 mRNA transcription while the knock in reporter systems were reporting IL-4 protein translation. The two knock in strains utilised in this thesis are the $\mathrm{G} 4$ and $\mathrm{KN} 2$ strains. Both reporter mice were constructed in a similar fashion: the KN2 mice had exons 1 and 2 of the Il4 gene replaced with an hCD2 reporter 


\section{Chapter One: General Introduction}

cassette while the G4 mice had exon 1 and 178 nt of intron 1 of the Il4 gene replaced with a GFP reporter cassette. This thesis made use of G4 and KN2 reporter mice, thereby analysing the expression of IL-4 protein expression and ensuring the reporting of differentiated Th2 cells.

These IL-4 reporter mice have enabled amazing breakthroughs in Th2 biology with a particular highlight involving a recent publication from our laboratory ${ }^{65}$. Here the G4 mice were utilized as a homozygous strain G4/G4, with both alleles expressing GFP reporter protein, resulting in an IL-4 deficient strain or heterozygous G4/IL-4, which remained sufficient in IL-4 while still producing GFP-IL-4 reporter protein. Elegant studies using multiple methods of inducing Th2 immune response in these homozygous G4 mice clearly depicted that there is no deficiency in the differentiation and development of Th2 when IL-4 is not present. A ground breaking study that challenges current dogma on the requirement of IL-4 for the development of Th2 cells and opens up multiple corridors of research into what potential factors could be responsible for the polarisation of Th2 cells.

Of course all systems have their limitations and cytokine reporter mice are not without their own. Of note are the different half-lives of reporter proteins versus their protein of interest half-life or even other reporter proteins half-lives. This can translate into false positives as a cell that is positive for a reporter protein may in real-time no longer be expressing the cytokine of interest and therefore when monitoring the cell the phenotype, location, interactions monitored could be altered from the time the cytokine was secreted. Secondly, altering the locus of a gene can affect the expression levels and binding abilities of transcription machinery and therefore alter the expression of the reporter proteins. Thirdly, expression of a reporter protein into the intracellular space may result in the cell becoming unstable and dying off earlier than a non-transgenic cell or even the complete opposite may occur with the cell becoming more stable and persisting for longer than a non-transgenic cell would. Therefore when using reporter mouse strains caution has to be taken when interpreting results as both the transcription of the gene of interest and the length of time the reporter protein remains are not necessarily the same as that of the protein of interest but only give an in situ snap shot of events that have occurred. 


\section{10 Research Objectives}

The exact requirements for the differentiation of Th2 cells, their dispersion and maintenance and the generation of effective Th2 memory cells remains uncertain. Not only do many of these fundamental properties about $\mathrm{Th} 2$ cell development remain unknown, the differentiated Th2 cell itself is inadequately characterised, with no distinct surface markers to recognise the Th2 cell population from the other helper cell subsets. The use of IL-4 protein reporters allowed us to map the differentiation and development of Th2 cells in vivo and investigate the role of an innate cytokine IL-25 in the differentiation and development of a Th2 response. Secondly upon crossing the two reporter mouse strains, the generation of a heterozygous IL-4 protein reporter mouse allowed us to investigate the regulation of IL-4 allelic expression and determine whether this regulation can be used as a biomarker for further understanding of the character of Th2 cells.

\section{Research Objective 1:}

To determine whether a newly described polarising cytokine, IL-25, is required for IL-4 independent differentiation of Th2 cells.

\section{Research Objective 2:}

To determine whether IL-25 is required for maintenance or recall responses mediated by IL-4 producing Th2 cells in vivo during the generation of protective immunity against parasitic helminths.

\section{Research Objective 3:}

To determine whether the allelic expression of the Il4 gene in CD4 T cells could be used to better characterise the Th2 subset and provide a clearer definition of an in vivo functional Th2 cell. 

Chapter Two:

Materials and Methods 
Chapter Two: Materials and Methods

2. 1 Labware

Table 2. 1 Labware

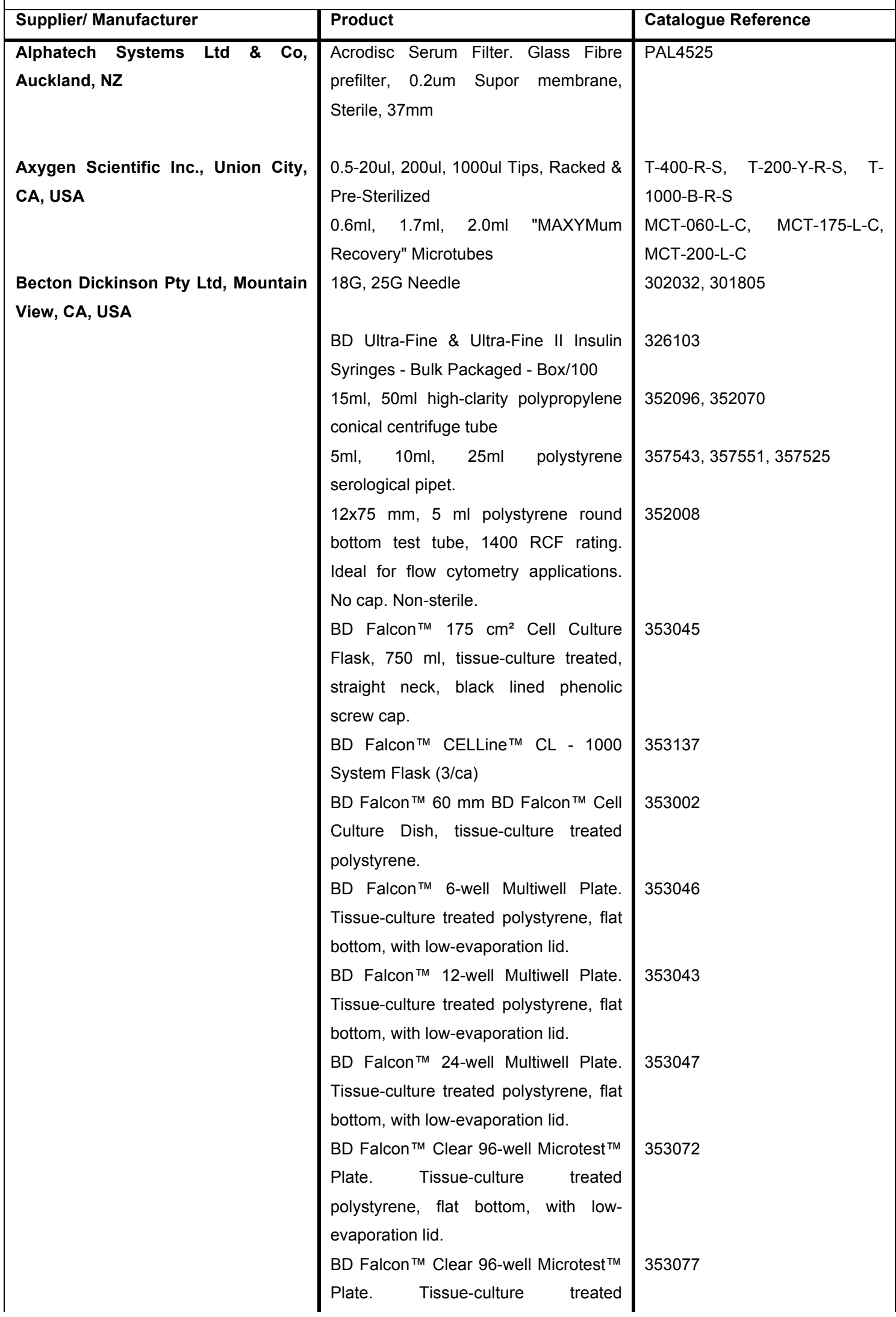




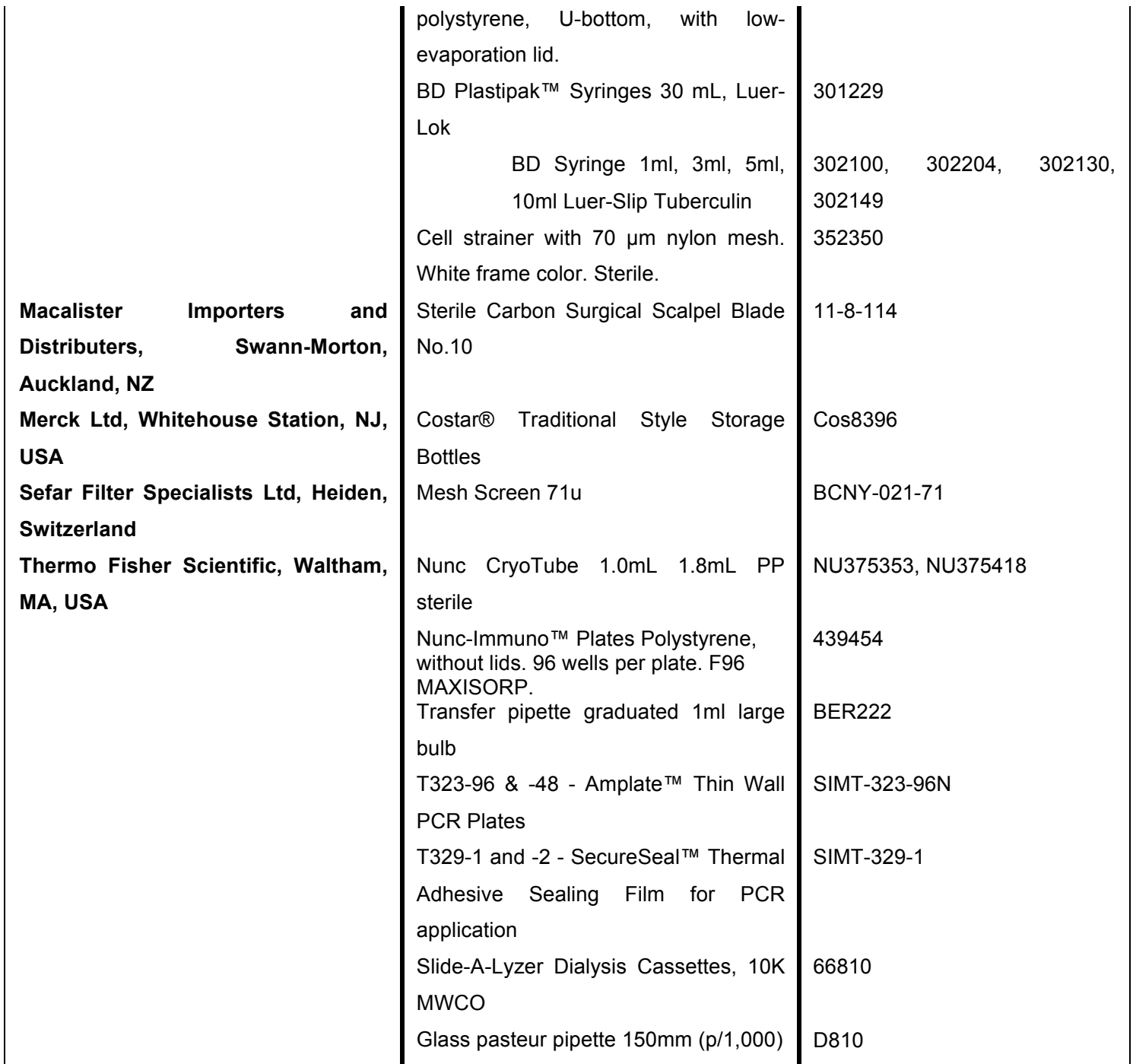




\section{2 Reagents and Buffers}

\subsection{2-mercaptoethanol (2 ME)}

$2 \mathrm{ME}$ was from Invitrogen (Auckland, NZ, 21985-023) and stored in aliquots at $4^{\circ} \mathrm{C}$.

\subsection{Alsever's Solution}

20.5 g Dextrose (BDH Laboratory Supplies, Poole, England), 8 g sodium citrate (BDH Laboratory Supplies, Poole, England) and $4.2 \mathrm{~g} \mathrm{NaCl}$ (Sigma, Auckland, NZ) were dissolved in distilled $1000 \mathrm{ml} \mathrm{MQ} \mathrm{H}_{2} \mathrm{O}$ (MilliQ) and the $\mathrm{pH}$ was adjusted to 6.1 with $1 \mathrm{M}$ citric acid (BDH Laboratory Supplies, Poole, England). Alsever's solution was stored at room temperature.

\subsection{Collagenase type I}

Collagenase I was purchased from Invitrogen (Auckland, NZ, 17100-017). The lyophilised powder was reconstituted in cIMDM to a concentration of $2.4 \mathrm{mg} / \mathrm{ml}$ and stored in aliquots at $-20^{\circ} \mathrm{C}$.

\subsection{DNase I}

DNase I was purchased as a lyophilised powder from Roche Diagnostics New Zealand Ltd, (Auckland, NZ, 10104159001) dissolved in cIMDM to a concentration of $10 \mathrm{mg} / \mathrm{ml}$ and stored at $-20^{\circ} \mathrm{C}$.

\subsection{Dimethyl sulfoxide (DMSO)}

DMSO was purchased from Sigma-Aldrich New Zealand Ltd (Auckland, NZ, D2650) and stored at room temperature.

\subsection{Ethylenediaminetetraacetic acid (EDTA)}

EDTA was purchased from Invitrogen (Auckland, NZ, 15575-020) and stored at room temperature until used. 


\subsection{Fluorescence Activated Cell Sorting (FACs) Buffer}

$0.01 \% \mathrm{NaN}_{3}$ and $2 \% \mathrm{FBS}$ were made up in PBS and stored at $4^{\circ} \mathrm{C}$.

\subsection{FACs Sort Buffer}

$0.002 \%$ DNase I and 2\% FBS were made up in PBS, filter sterilised and stored at $4^{\circ} \mathrm{C}$.

\subsection{Foetal Bovine Serum (FBS)}

FBS was purchased from Invitrogen (GIBCO, Auckland, NZ, 12203C) and stored in $25 \mathrm{ml}$ aliquots at $-20^{\circ} \mathrm{C}$.

\subsection{Complete Iscove's Modified Dulbecco's Medium (cIMDM)}

IMDM was purchased from Invitrogen (Auckland, NZ, 31980-097) and supplemented with $100 \mathrm{U} / \mathrm{ml}$ Penicillin-Streptomycin from Invitrogen (Gibco, Auckland, NZ, 15140-122), $55 \mu \mathrm{M} 2 \mathrm{ME}$ and 5\% FBS. Media was filter sterilised and stored at $4^{\circ} \mathrm{C}$ for a maximum of 14 days.

\subsection{Lipopolysaccharides (LPS)}

LPS from Escherichia coli, serotype 0111:B4, was purchased as a lyophilised powder from Sigma-Aldrich New Zealand Ltd (Auckland, NZ, L4391), dissolved in IMDM to a stock concentration of $1 \mathrm{mg} / \mathrm{ml}$ and stored at $4^{\circ} \mathrm{C}$. The stock concentration was further diluted in IMDM to a working solution of $10 \mu \mathrm{g} / \mathrm{ml}$, which was used immediately.

\subsection{Lung Digestion Buffer}

Collagenase type I at a final concentration of $2.4 \mathrm{mg} / \mathrm{ml}$ from stock at $24 \mathrm{mg} / \mathrm{ml}$ and DNAse 1 at $120 \mu \mathrm{g} / \mathrm{ml}$ from a stock of $10 \mathrm{mg} / \mathrm{ml}$ were made up in cIMDM to the required volume.

\subsection{Magnetic Cell Sorting (MACS) Running Buffer}

2 mM EDTA, 0.5\% BSA and 0.012\% DNase I were made up in PBS, filter sterilised and stored at $4^{\circ} \mathrm{C}$. 
Chapter Two: Materials and Methods

\subsection{Penicillin-Streptomycin}

Penicillin-Streptomycin was purchased in liquid form from Invitrogen (Gibco, Auckland, NZ, 15140-122) and stored as single use aliquots at $4^{\circ} \mathrm{C}$ until used.

\subsection{Phosphate Buffered Saline (PBS)}

PBS powder containing no calcium or magnesium was purchased from Invitrogen (Gibco, Auckland, NZ, 21600-069) and dissolved in $\mathrm{dH}_{2} \mathrm{O}$.

\subsection{Red Blood Cell Lysing Buffer Hybri-Max ${ }^{\text {rm }}$ (RBC)}

RBC Lysing Buffer was purchased from Sigma-Aldrich New Zealand Ltd (Auckland, NZ, R7757) and stored at room temperature.

\subsection{Sodium Azide $\left(\mathrm{NaN}_{3}\right)$}

$\mathrm{NaN}_{3}$ was purchased in powder form from Sigma-Aldrich New Zealand, Ltd (Auckland, NZ, S8032) and dissolved in PBS to give a stock concentration of 5\%. The solution was stored at room temperature.

\subsection{Tween $^{\circledR} 20$ (Tween)}

Tween was purchased from Sigma-Aldrich New Zealand Ltd (Auckland, NZ, P2287) and stored at room temperature until used.

\subsection{Trypan Blue Stain (Life Technologies ${ }^{\mathrm{TM}}$ )}

Trypan Blue was purchased from Invitrogen (Auckland, NZ, 15250-061) and stored at room temperature. 


\section{3 Cytokines}

\subsection{Fms-like tyrosine kinase ligand (rFlt3L)}

A chinese hamster ovary $(\mathrm{CHO})$ cell line that was modified to secrete Flt3L ${ }^{191}$ was kindly supplied by Prof Nic Nicola (WEHI, Melbourne, Australia). The cell line was cultured and supernatants containing Flt3L were harvested and filtered through a 0.2 $\mu \mathrm{M}$ serum filter. The Flt3L supernatant was titrated using BMDC cultures to select for optimal concentration as determined by cell recovery and clear separation of DC phenotypes, this determined concentration was used for future BMDC culture set ups. Aliquots were stored at $-80^{\circ} \mathrm{C}$ and working aliquots were kept at $4^{\circ} \mathrm{C}$ for no longer than 1 week.

\subsection{Granulocyte-macrophage colony stimulating factor (rGM-CSF)}

Murine X63 cell line was cultured to produce the full-length murine GM-CSF protein. The harvested GM-CSF containing supernatant was tested using BMDC cultures. Aliquots were stored at $-80^{\circ} \mathrm{C}$ and working aliquots were kept at $4^{\circ} \mathrm{C}$ for no longer than 1 week.

\subsection{Interleukin -2 (rIL-2)}

X62-IL-2 cell line was cultured to produce recombinant murine IL-2. Supernatants containing IL-2 were tested using IL-2 dependent cell lines (HT2) to determine bioactivity. Aliquots were stored at $-80^{\circ} \mathrm{C}$ and working aliquots were kept at $4^{\circ} \mathrm{C}$ for no longer than 1 week.

\subsection{Interleukin -4 (rIL-4)}

Chinese Hamster Ovary cell line CHO-mIL-4 was cultured to produce full-length murine IL-4 protein. Supernatants containing IL-4 were tested using IL-4 dependent cell lines (CT.4S) to determine bioactivity. Aliquots were stored at $-80^{\circ} \mathrm{C}$ and working aliquots were kept at $4^{\circ} \mathrm{C}$ for no longer than 1 week. 


\subsection{Interleukin 25 (rIL-25)}

Recombinant mouse IL-25 was purchased as a lyophilized powder from R\&D Systems (Minneapolis, MN, USA, 1399-IL) and reconstituted at $10 \mu \mathrm{g} / \mathrm{ml}$ in sterile 4 mM HCL containing 0.1\% FBS the further diluted in PBS to make up a stock solution of $1 \mu \mathrm{g} / \mathrm{ml}$ and stored in aliquots at $-80^{\circ} \mathrm{C}$.

\section{4 Allergens and Peptides}

\subsection{ISQ peptide}

Ova peptide ISQAVHAAHAEINEAGR $\left(\mathrm{OVA}^{323-339}\right)$ was purchased from Mimotopes Pty Ltd (Clayton, VA, Australia) and referred to as ISQ peptide. Lyophilized protein was reconstituted in PBS to $1 \mathrm{mM}$ and stored at $-80^{\circ} \mathrm{C}$.

\subsection{House Dust Mite (HDM)}

HDM (Dermatophagoides Pteronyssinus) was purchased from Greer (Lenoir, N.C., USA, XPB82D3A2.5) and reconstituted in sterile PBS to $100 \mu \mathrm{g} / 30 \mu \mathrm{l}$ and stored at $-80^{\circ} \mathrm{C}$.

\subsection{Dead L3 N. brasiliensis}

L3 N. brasiliensis larvae were washed three times in sterile PBS placed into aliquots with a concentration of $600 \mathrm{~L} 3 \mathrm{~N}$. brasiliensis/ $30 \mu \mathrm{l}$ and put through three freezethaw cycles. Larvae were checked for loss of viability through heat exposure and stored at $-80^{\circ} \mathrm{C}$. 


\section{5 Antibodies and Fluorophores}

\subsection{4,6-diamidino-2-phenylindole, dihydrochloride (DAPI)}

DAPI was purchased as a lyophilised powder from Invitrogen (Auckland, NZ, D1306), dissolved in $\mathrm{dH}_{2} \mathrm{O}$ to a stock concentration of $5 \mathrm{mg} / \mathrm{ml}$ and stored in aliquots at $-80^{\circ} \mathrm{C}$. The stock solution was further diluted in FACs buffer to a working solution of $200 \mathrm{mg} / \mathrm{ml}$ and stored protected from light at $4^{\circ} \mathrm{C}$. Samples were incubated just prior to acquisition with $40 \mu \mathrm{g}$ DAPI.

\subsection{7-Amino-Actinomycin (7-AAD)}

7-AAD was purchased from Becton Dickinson Pty Ltd (Auckland, NZ, 559925) and stored protected from light at $4^{\circ} \mathrm{C}$. Samples were incubated just prior to analysis with $0.25 \mu \mathrm{g}$ 7-AAD for 10 minutes, washed in $3 \mathrm{ml}$ FACS buffer and acquired.

\subsection{AbC $^{\mathrm{TM}}$ Anti-Rat/Hamster Bead Kit (Molecular Probes ${ }^{\circledR}$ )}

AbC compensation beads were purchased from Invitrogen (Auckland, NZ, A10389) and stored protected from light at $4^{\circ} \mathrm{C}$. Capture beads (Component A) were resuspended for 30 seconds just prior to adding a droplet to each compensation tube. Beads were stained for 15 minutes at appropriate antibody concentration, washed in 3 ml FACS buffer and resuspened in $500 \mu \mathrm{l}$ FACS buffer. Finally a droplet of resuspended negative beads (Component B) was added.

\subsection{Anti-Mouse Ig, $k /$ Negative Control (FBS) Compensation Particles Set}

Anti-mouse compensation beads were purchased from Becton Dickinson Pty Ltd (Auckland, NZ, 552843) and stored protected from light at $4^{\circ} \mathrm{C} . \mathrm{BD}^{\mathrm{TM}}$ CompBeads Anti-Mouse Ig, $\kappa$ beads were resuspended for 30 seconds just prior to adding a droplet per compensation tube. Beads were stained for 15 minutes at appropriate antibody concentration, washed in $3 \mathrm{ml}$ FACS buffer and resuspened in $500 \mu \mathrm{l}$ FACS buffer. Finally a droplet of resuspended BD ${ }^{\mathrm{TM}}$ CompBeads Negative Control (FBS) was added. 


\subsection{LIVE/DEAD ${ }^{\circledR}$ Fixable Blue Dead Cell Stain Kit (Molecular Probes ${ }^{\circledR}$ )}

Fixable Blue was purchased from Invitrogen (Auckland, NZ, L-23105) and stored protected from light at $-20^{\circ} \mathrm{C}$. Once at room temperature $50 \mu \mathrm{l}$ anhydrous DMSO (Component B) was added to an aliquot of the fluorescent reactive dye (Component A) to form a working solution. $1 \mu \mathrm{l}$ working solution/ $1 \mathrm{ml}$ PBS was used to stain each sample for 30 minutes at $4^{\circ} \mathrm{C}$, samples were then washed in $2 \mathrm{ml}$ PBS whereby normal process for staining for surface markers was followed.

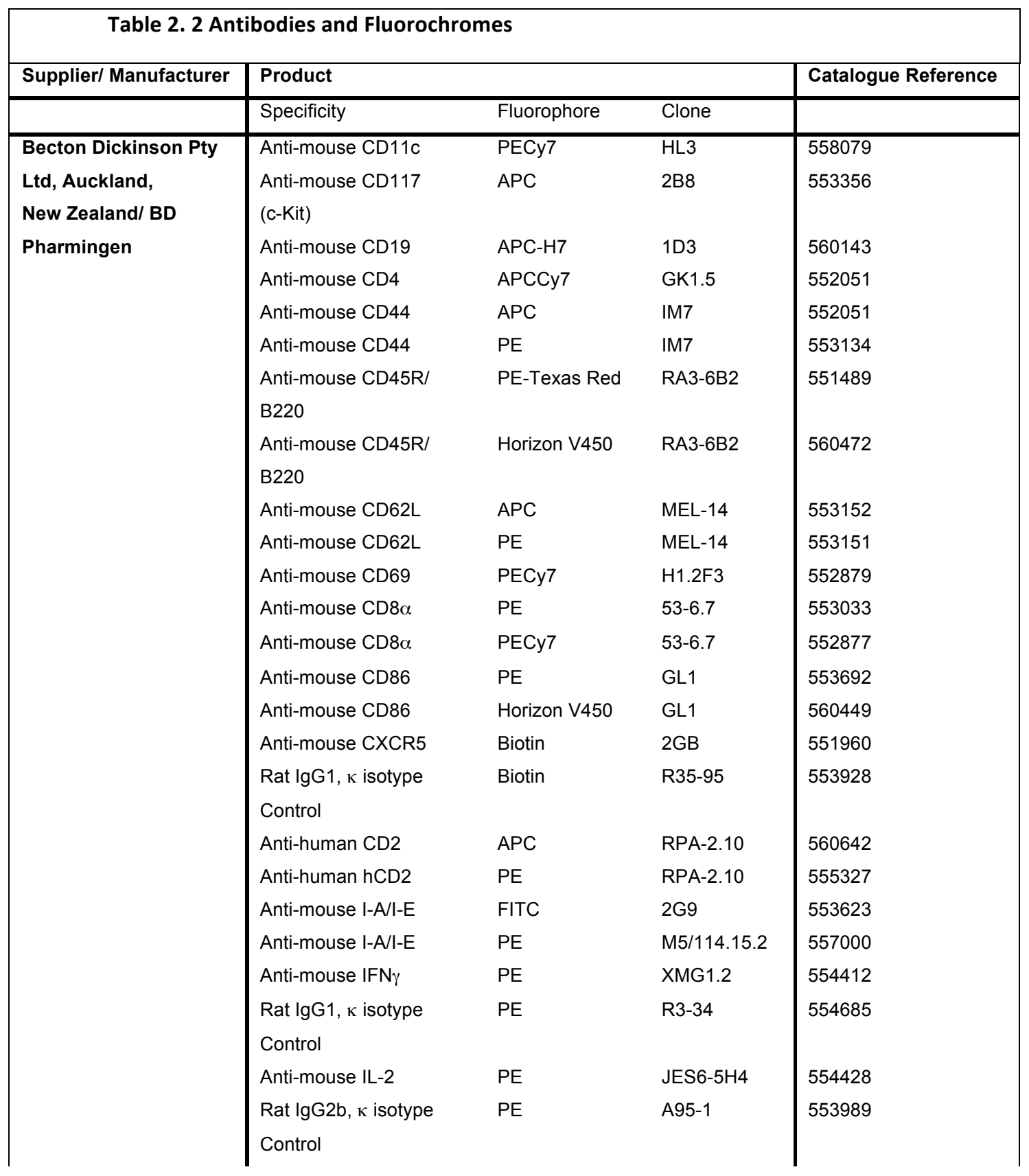


Chapter Two: Materials and Methods

\begin{tabular}{|c|c|c|c|c|}
\hline & Anti-mouse IL-3 & PE & MP2-8F8 & 554383 \\
\hline & Anti-mouse IL-4 & APC & 11B11 & 554436 \\
\hline & Rat $\lg G 1, \kappa$ isotype & APC & R3-34 & 554686 \\
\hline & Control & & & \\
\hline & Anti-mouse IL-4 & PE & 11B11 & 554435 \\
\hline & Biotin & APC & Streptavidin & 349024 \\
\hline & Biotin & APCCy7 & Streptavidin & 554063 \\
\hline & Biotin & PE & Streptavidin & 554061 \\
\hline & Siglec-F & PE & $\mathrm{E} 50-2440$ & 552126 \\
\hline & Anti-mouse $V_{\alpha 2}$ & APC & B20.1 & 560622 \\
\hline & Anti-mouse V $\beta 5.1 / 5.2$ & PE & MR9-4 & 553190 \\
\hline \multirow{7}{*}{$\begin{array}{l}\text { Norrie Biotech, } \\
\text { Auckland, NZI } \\
\text { Biolegend }\end{array}$} & Anti-mouse CD11b & Biotin & $\mathrm{M} 1 / 70$ & 101204 \\
\hline & Anti-mouse CD11b & PerCP & $\mathrm{M} 1 / 70$ & 101230 \\
\hline & $\begin{array}{l}\text { Anti-mouse CD127 } \\
\text { (IL-7R } \alpha)\end{array}$ & PECy7 & A7R34 & 135014 \\
\hline & $\begin{array}{l}\text { Anti-mouse CD279 } \\
\text { (PD1) }\end{array}$ & PECy7 & RMP1-30 & 109110 \\
\hline & Anti-mouse CD45 & Pacific Blue & $30-F 11$ & 103126 \\
\hline & Anti-mouse CD45 & APCCy7 & C363-16A & 103310 \\
\hline & Anti-mouse CD62L & Pacific Blue & MEL-14 & 104424 \\
\hline \multirow{14}{*}{$\begin{array}{l}\text { Huntingtree } \\
\text { Bioscience, Auckland, } \\
\text { NZ/ eBioscience }\end{array}$} & Anti-mouse BCL6 & PE & mGL191e & EBI-12-9887-80 \\
\hline & $\begin{array}{l}\text { Mouse IgG1 K isotype } \\
\text { Control }\end{array}$ & PE & P3.6.2.8.1 & EBI-12-4718-81 \\
\hline & Anti-mouse $\mathrm{CD} 3 \varepsilon$ & PECy7 & $2 \mathrm{C} 11$ & $25-0031-81$ \\
\hline & $\begin{array}{l}\text { Anti-mouse CD49b } \\
\text { (pan-NK) }\end{array}$ & PECy7 & DX5 & $25-5971-82$ \\
\hline & Anti-mouse FceR1 $\alpha$ & Biotin & MAR-1 & $13-5898-85$ \\
\hline & Anti-mouse FceR $1 \alpha$ & $\mathrm{PE}$ & MAR-1 & $12-5898-83$ \\
\hline & Anti-mouse GATA3 & PerCPeF710 & TWAJ & EBI-46-9966-41 \\
\hline & $\begin{array}{l}\text { Rat IgG2b } \kappa \text { Isotype } \\
\text { Control }\end{array}$ & PerCPeF710 & & EBI-46-4031-80 \\
\hline & Anti-mouse IL-5 & $\mathrm{PE}$ & TRFK5 & $12-7052-82$ \\
\hline & $\begin{array}{l}\text { Rat IgG1, } \kappa \text { Isotype } \\
\text { Control }\end{array}$ & PE & & $12-4301-83$ \\
\hline & Anti-mouse IL-13 & AF647 & eBio13A & $51-7133-80$ \\
\hline & $\begin{array}{l}\text { Rat IgG1, } \kappa \text { Isotype } \\
\text { Control }\end{array}$ & AF647 & & $51-4301-80$ \\
\hline & Anti-mouse IL-13 & $\mathrm{PE}$ & eBio13A & $12-7133-82$ \\
\hline & Biotin & PECy7 & Streptavidin & EBI-25-4317-82 \\
\hline \multirow{3}{*}{$\begin{array}{l}\text { Invitrogen, Auckland, } \\
\text { NZ } \\
\text { Malaghan Institute of } \\
\text { Medical Research }\end{array}$} & Anti-mouse CD $3 \varepsilon$ & AF488 & $500 A 2$ & HM3420 \\
\hline & Anti-mouse CD4 & QDot 605 & RM4-5 & Q10092 \\
\hline & $\begin{array}{l}\text { Anti-mouse CD16/CD32 } \\
\text { (Fcyll//II Receptor) }\end{array}$ & & $2.4 \mathrm{G} 2$ & \\
\hline
\end{tabular}




\section{6 Mice}

\subsection{Maintenance and Ethics Approvals}

Mice were bred and maintained in the Biomedical Research Unit of the Malaghan Institute of Medical Research. Mice were age and sex matched and experimental procedures were approved by the Victoria University Animal Ethics Committee and carried out in accordance with the guidelines of Victoria University of Wellington, New Zealand.

\subsection{Mouse Strains}

${\mathrm{IL}-4^{+/+}} \mathrm{BALB} / \mathrm{c}$ breeding pairs were obtained from the Jackson Laboratories (Bar Harbour, ME, USA). STAT6 ${ }^{-/}$(on BALB/c background) breeding pairs were obtained from mice originally from Kaplan et al. ${ }^{192}$. IL-4 ${ }^{+/+}$C57BL/6 breeding pairs were obtained from the Jackson Laboratories (Bar Harbour, ME, USA). eGFP (on C57BL/6 background) mice were obtained from the Hercus Taieri Research Unit, Dunedin, NZ ${ }^{193} . \mathrm{G}^{+/-}$(on C57BL/6 and BALB/c background) breeding pairs were obtained from W.E. Paul (National Institute of Allergy and Infectious Diseases, National Institutes of Health, Bethesda, MD, USA) ${ }^{119}$. IL-25 $5^{-/-}$(on C57BL/6 background) breeding pairs were obtained from M. Kleinschek (SP Biopharma, CA, USA) ${ }^{194} . \mathrm{KN}^{+/+}$(on BALB/c background) breeding pairs were obtained from R. Locksley (University of California San Francisco, CA, USA) ${ }^{190}$. OTII mice express transgenic TCR specific for ISQAVHAAHAEINEAGR (OVA ${ }^{323-339}$ ) (on C57BL/6 background), were obtained with the permission of Prof. Frank Carbone, Melbourne University, Australia ${ }^{195}$.

\subsection{Genotyping $\mathrm{G}^{+/+} \mathrm{IL}-25^{-/-}$mice}

To determine whether signalling by IL-25 is necessary for in vivo Th2 differentiation, a Th2 reporter IL-25 deficient in vivo system was developed. $\mathrm{G}^{+/+}$reporter mice were crossed with IL-25 $5^{-/}$mice to create an F1 heterozygous $\mathrm{G} 4^{+/-} \mathrm{IL}-25^{+/-}$heterozygous strain. These F1 generation mice were crossed against other F1 generation mice and progeny were selected for global deficiency in IL-25. Heterozygous $\mathrm{G}^{+/-}$mice provided an IL-4 sufficient system and homozygous $\mathrm{G}^{+/+}$mice an IL-4 deficient system, effectively generating a double cytokine deficient mouse. 
Genotyping was performed on DNA extracted from tail cuts. Tails were hydrolyzed in $50 \mathrm{mM} \mathrm{NaOH}$ (BDH Laboratory Supplies, Poole, England) at $95^{\circ} \mathrm{C}$ for 1 hour to extract DNA. PCR was performed with primers from Sigma GenoSys (Sigma Aldrich, Auckland, NZ) using the i-Taq ${ }^{(\mathrm{TM})}$ DNA Polymerase kit (iNtRON Biotechnology, Korea, 25022). PCR products were run on 1\% Agarose gel (BDH Laboratory Supplies, Poole, England), loaded with $10 \mathrm{X}$ BlueJuice ${ }^{\mathrm{TM}}$ Gel Loading Buffer (Invitrogen $^{\mathrm{TM}}$, Auckland, NZ, 10816-015) and visualised with SYBR ${ }^{\circledR}$ Safe DNA Gel Stain (Invitrogen ${ }^{\mathrm{TM}}$, Auckland, NZ, S33102). TrackIt ${ }^{\mathrm{TM}} 1 \mathrm{~Kb}$ Plus DNA Ladder (Invitrogen $^{\mathrm{TM}}$, Auckland, NZ, 10488-085) was used to determine PCR product sizes as depicted in Figure 2. 1.

Gene target: IL-4

Il4 gene yields a 688 bp product with primers a and b;

gfp gene yields $1600 \mathrm{bp}$ product with primers $\mathrm{a}$ and $\mathrm{b}$

a, 5'-GTC TGA AAG GCC GAT TAT GG-3' (Rev musIL-4.N5)

b, 5'-GTT AAT CCA AGC AGG ACA GAG-3' (For musIL-4.N6)

Gene target: GFP

$g f p$ gene yields a $550 \mathrm{bp}$ product with primers $\mathrm{c}$ and $\mathrm{b}$

c, 5'-GGG ATC ACT CTC GGC ATG GAC-3' (Rev. eGFP.N2)

b, 5'-GTT AAT CCA AGC AGG ACA GAG-3' (For. musIL-4N6)

Gene target: IL-25 WT

Wild type $i l 25$ gene yields 300 bp product with primers $\mathrm{d}$ and e;

d, 5'-CCTGCTGCTTCAGGTAGGGCTTTG-3' (Rev.)

e, 5'-CTACAGACAGGCTCCCACATGGACC-3' (For.)

Gene target: IL-25KO

Knock out lacZ gene yields 770 bp product with primers $\mathrm{f}$ and $\mathrm{g}$;

f, 5'-CCTGCTGCTTCAGGTAGGGCTTTG-3' (Rev.)

g, 5'-GCTGACTCTCAACATTCTACTCCTCC-3' (For.) 


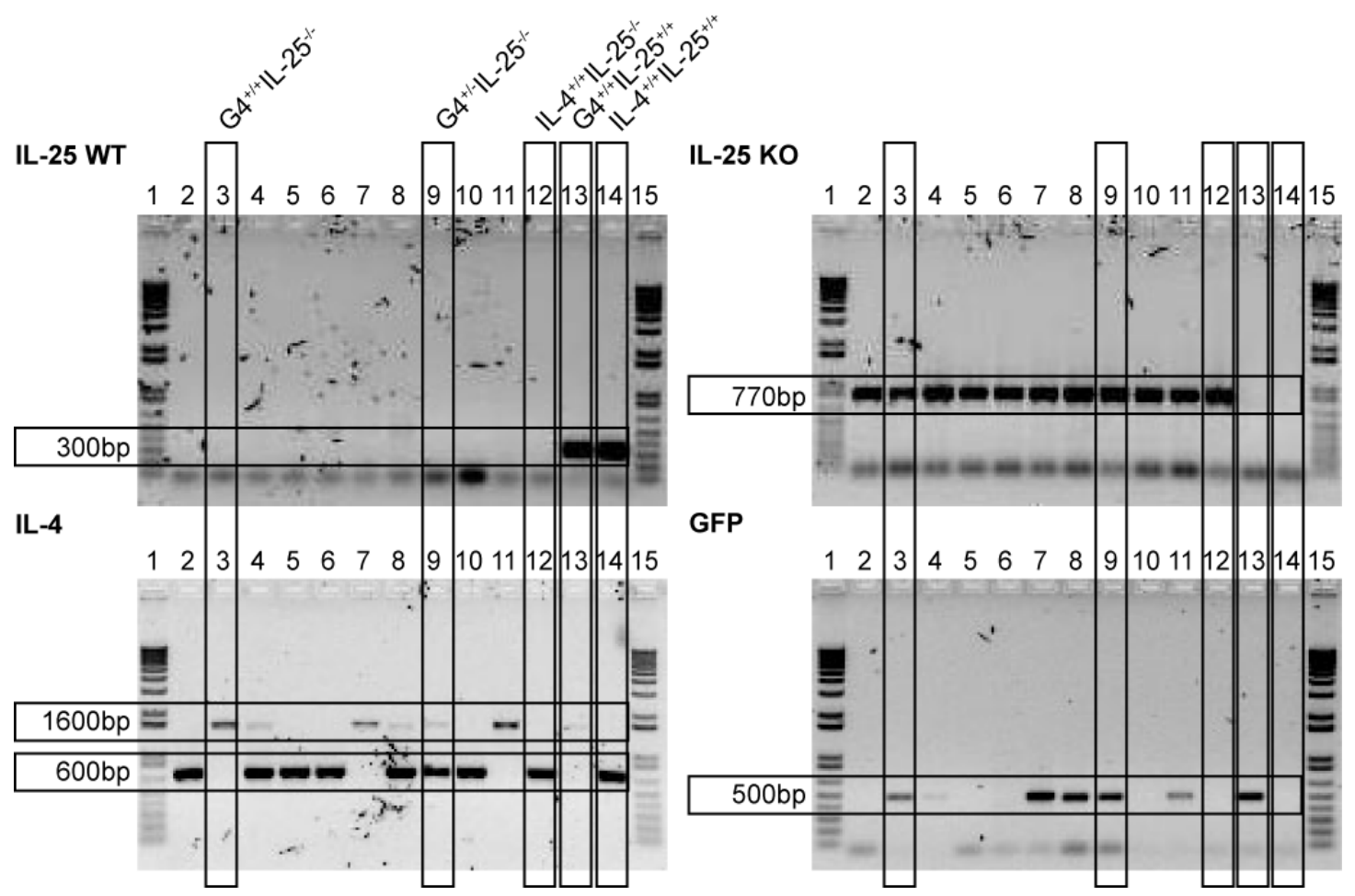

Figure 2. 1 Genotyping of IL-4 reporter mice ${ }^{+/+} \mathrm{IL}-25^{-/-}$mice. DNA was extracted from tail cuts of individually marked pups. DNA was prepared and the PCR products run on an agar gel. IL25 WT gel: 300 bp segment illustrating intact IL-25 gene. IL-25 KO gel: 770 bp fragment illustrating LacZ insertion and deletion of IL-25. IL-4 gel: 1600 bp fragment indicating GFP insertion in IL-4 allele while 600 bp denotes WT IL-4 allele. GFP gel: 500 bp product denotes GFP insertion into IL-4 allele. Column 3: $\mathrm{G}^{+/+} \mathrm{IL}-25^{-/-}$; Column 9: G4 ${ }^{+/-} \mathrm{IL}-25^{-/-}$; Column 12: IL$4^{+/+} \mathrm{IL}-25^{-/}$; Column 13: G4 $4^{+/+} \mathrm{IL}-25^{+/+}$; Column 14: ${\mathrm{IL}-4^{+/+}}_{\mathrm{IL}-25^{+/+}}$.

\subsection{Genotyping OTII and OTII G4 ${ }^{+/+}$mice}

A lateral tail vein bleed of a few droplets of blood into $500 \mu \mathrm{l}$ of Alsever's solution from each naïve animal was washed at $650 \mathrm{~g}$ for $2 \mathrm{~min}$. Red blood cells were lysed in $500 \mu \mathrm{dH}_{2} \mathrm{O}$ for 30 seconds and topped up with $500 \mu \mathrm{l} 1.8 \% \mathrm{NaCl}$ and washed at 650 g for 2 min. Samples were blocked with 2.4G2 and stained for CD4 Fitc, V $\beta$ 5.1/5.2 PE and V $\alpha 2$ APC and analysed on the BD FACSCalibur (Becton Dickinson, San Jose, CA). 


\section{7 Drug Treatments}

\subsection{Anti-helminthic}

Noromectin worming paste (Drench, Lawrence, NZ), with active ingredient ivermectin, was administered by oral gavage of $20 \mathrm{mg} / \mathrm{kg}$ body weight in $0.2 \mathrm{ml}$ total volume. Dose was administered twice, 5 days apart.

\subsection{Anaesthetic}

10x stock solution was diluted in sterile PBS to $8.6 \mathrm{mg} / \mathrm{ml}$ ketamine and $0.26 \mathrm{mg} / \mathrm{ml}$ xylazine working solution. The anaesthetic was injected into the peritoneal cavity (i.p.) at $10 \mu \mathrm{l} / \mathrm{g}$ of mouse for a restraint dose and $50 \mu \mathrm{l} / \mathrm{g}$ of mouse for lethal dose.

\section{8 Methods}

\subsection{In vitro culture systems}

\subsubsection{Polyclonal activation of CD4 T cells}

Spleens, lymph nodes or thymi were collected from euthanized naïve mice and processed through a $70 \mu \mathrm{m}$ nylon filter into a single cell solution in cIMDM. CD4 T cells with positively selected using Dynabeads FlowComp ${ }^{\mathrm{TM}}$ Mouse CD4 (Invitrogen, Auckland, NZ, 114.61D) and incubated for 3-5 days in 24 well plates at $2 \times 10^{6}$ cells/ well on plate bound $\alpha \mathrm{CD} 3(0.05,0.2,1$ or $10 \mu \mathrm{g} / \mathrm{ml})$ with soluble $\alpha \mathrm{CD} 28$ supernatant (1:50) and rIL-2 (50 U/ ml) with or without rIL-4 (1000 U/ ml) in $1 \mathrm{ml}$ cIMDM at $37^{\circ} \mathrm{C}$ and $5 \% \mathrm{CO}_{2}$. Half the culture supernatant was replaced with fresh cIMDM and cytokines at $2 \mathrm{X}$ concentration at 3 days and cells were harvested and analysed by flow cytometry at 5 days after culture.

\subsubsection{Generation of bone marrow derived GMCSF dendritic Cells}

$\mathrm{G} 4^{+/+}$mice were euthanised and the hind legs were detached at the hip and all soft tissue was removed and the bones were placed into IMDM. The knee joints and ends of the femur and tibia were cut off and the bone marrow was flushed out into a $50 \mathrm{ml}$ 
Falcon tube. Cell clumps were disrupted by vigorous repeat pipetting with the cell suspension passed through a $70 \mu \mathrm{m}$ nylon filter. Live cells were identified by trypan blue exclusion dye (GIBCO, Invitrogen, Auckland, NZ) and counted using a haemocytometer. Cells were pelleted by centrifugation at $400 \mathrm{~g}$ for 4 minutes and resuspended at $4 \times 10^{6}$ cells $/ \mathrm{ml}$. Cells were plated out into 6 well plates at $2 \times 10^{6}$ cells/ well containing $10 \mathrm{ng} / \mathrm{ml} \mathrm{rGMCSF}$ and $20 \mathrm{ng} / \mathrm{ml} \mathrm{rIL}-4$. Cells were incubated at $37^{\circ} \mathrm{C}$ and $5 \% \mathrm{CO}_{2}$ and cytokines were replenished on days 2,4 and 6 by replacing 2.5 $\mathrm{ml}$ from each well with $2.5 \mathrm{ml}$ of cIMDM containing $20 \mathrm{ng} / \mathrm{ml}$ GM-CSF and $40 \mathrm{ng} /$ $\mathrm{ml}$ IL-4.

\subsubsection{Generation of bone marrow derived Flt3L dendritic Cells}

Bone marrow was obtained as described in 2.8.1. 2 and cells were plated in CIMDM into 6 well plates at $5 \times 10^{6}$ cells/ well containing appropriate concentration of Flt3L supernatant as determined in section 2.3. 1. Cells were incubated at $37^{\circ} \mathrm{C}$ and $5 \% \mathrm{CO}_{2}$ and Flt3L was replenished on days 3, 6 and 9 by replacing $2.5 \mathrm{ml}$ from each well with $2.5 \mathrm{ml}$ of cIMDM containing 2x Flt3L supernatant at working concentration.

\subsubsection{Activation of dendritic Cells}

At day 6 for the remaining 18 hours of the GMCSF BMDC culture or day 9 of the Flt3L BMDC culture, the BMDCs were activated with $100 \mathrm{ng} / \mathrm{ml}$ LPS or $125 \mathrm{ng} / \mathrm{ml}$ rIL-25. Adherent cells were harvested the following day by gently rinsing the plates with the supernatant 2- 3 times before collecting the supernatant.

\subsubsection{BMDC activation of OTII T cells}

ISQ peptide was loaded onto activated GMCSF or Flt3L BMDCs by incubating peptide from concentration of $0.001-10 \mu \mathrm{M}$ and BMDCS in $50 \mathrm{ml}$ Falcon tubes for 4 hours $37^{\circ} \mathrm{C}$ and $5 \% \mathrm{CO}_{2}$. Spleens were collected from euthanized naïve $\mathrm{G}^{+/+}$OTII or OTII mice and processed through a $70 \mu \mathrm{m}$ nylon filter into a single cell solution in cIMDM. CD4 T cells with positively selected using Dynabeads ${ }^{\circledR}$ FlowComp ${ }^{\mathrm{TM}}$ Mouse CD4 (Invitrogen, Auckland, NZ, 114.61D) and incubated with $0.5 \times 10^{6}$ GMCSF or Flt3L BMDCs/ well and $2 \times 10^{6} \mathrm{CD} 4 \mathrm{~T}$ cells/ well for 3 days in 12 well plates with rIL$2(50 \mathrm{U} / \mathrm{ml})$ with or without rIL-4 $(1000 \mathrm{U} / \mathrm{ml})$ with or without $\alpha \mathrm{IL}-12(10 \mu \mathrm{g} / \mathrm{ml})$ 
with or without rIL-12 (10 ng/ml) in $3 \mathrm{ml} \mathrm{cIMDM}$ at $37^{\circ} \mathrm{C}$ and $5 \% \mathrm{CO}_{2}$. Following 3 days in culture, cells were harvested and analysed by flow cytometry.

\subsubsection{C57BL/6 BMDC activation of BALB/C CD4 T cells}

$1 \times 10^{6} \mathrm{GMCSF}$ C57BL/6 $\mathrm{IL}^{-/-} \mathrm{BMDC} /$ well were incubated along with $2 \times 10^{6}$ $\mathrm{BALB} / \mathrm{c} \mathrm{G}^{+/+} \mathrm{STAT6}^{+/+}$or BALB/c G4 ${ }^{++} \mathrm{STAT6}^{-/-} \mathrm{CD} 4 \mathrm{~T}$ cells/ well for 3 days in 12 well plates in the presence of rIL-2 $(50 \mathrm{U} / \mathrm{ml})$ with/ without rIL-4 $(1000 \mathrm{U} / \mathrm{ml})$ in $3 \mathrm{ml}$ cIMDM at $37^{\circ} \mathrm{C}$ and $5 \% \mathrm{CO}_{2}$. Following 3 days in culture, cells were harvested and analysed by flow cytometry.

\subsection{In vivo assay for differentiation of Th2 cells}

Mice were anaesthetised and $30 \mu$ l of sterile PBS containing 600 dead L3 N. brasiliensis or $100 \mu \mathrm{g}$ HDM was injected into the dermis of the ear using a BD Ultrafine 29 gauge needle. The singular auricular lymph node was harvested between 3 and 7 days later into PBS and processed through a $70 \mu \mathrm{M}$ cell strainer into a single cell suspension, ready to be prepared and analysed by flow cytometry.

\subsubsection{Helminth model: Nippostrongylus brasiliensis}

\subsubsection{1 Maintenance of the Parasite}

$N$. brasiliensis was maintained by passage through Wistar rats. 5000 infectious stage L3 larvae per $500 \mu \mathrm{l}$ PBS were subcutaneously (SQ) injected into rats. Faeces were collected from days 7-10 post inoculation. Faecal pellets were softened and mixed with granulated charcoal and placed in the centre of dampened Whatman filter paper allowing eggs to hatch and migrate to the edges of the petri dish. Faecal cultures were kept moist at $25^{\circ} \mathrm{C}$ and L3 larvae was harvested from 7 days to 28 days and used in infection studies.

\subsubsection{2 Primary and Challenge Infection Models}

L3 infectious stage larvae were harvested from faecal cultures and washed 3 times in $50 \mathrm{ml}$ sterile PBS. Larvae were re-suspended $600 \mathrm{~L} 3 / 200 \mu 1$ doses and administered 
SQ to the nape of the neck. In experiments involving IL-25 deficient animals, challenge infections of $600 \mathrm{~L} 3 / 200 \mu \mathrm{l}$ were administered SQ at day 45 after primary infection. All other challenge infections occurred 30 days post primary infection. At indicted times, animals were sacrificed by administration of lethal anaesthetic.

\subsubsection{3 Worm burdens}

At indicated days following primary or challenge infections either lungs for L4 stage larvae or small intestines for L5 stage larvae were removed from the sacrificed animal. The tissues were finely minced, placed on cheesecloth and suspended at the surface of a $50 \mathrm{ml}$ tube containing PBS at $37^{\circ} \mathrm{C}$ for 2 hour for small intestines and overnight for lungs. Viable worms were allowed to migrate out of minced tissue sections and collect at the bottom of the tube where they were removed and enumerated under the inverted microscope.

\subsubsection{Helminth model: Heligmosomoides polygyrus}

\subsubsection{1 Maintenance of the Parasite}

H. polygyrus was maintained by passage through $\mathrm{STAT}^{-/}$mice. 200 infectious stage L3 larvae per $200 \mu \mathrm{l}$ PBS were administered intragastrically (i.g.). Faeces from infected mice were collected anywhere from 14 days to 4 months post inoculation (PI). Faecal pellets were softened and emulsified in $\mathrm{dH}_{2} 0$, large debris were removed by passing through a sieve, centrifuged at $500 \mathrm{~g} / 2 \mathrm{~min}$ and slurry was placed in the centre of dampened Whatman filter paper to allow eggs to hatch. Faecal cultures were kept moist at $25^{\circ} \mathrm{C}$ and L3 larvae was harvested from 7 days to 9 days washed in sterile $\mathrm{dH}_{2} \mathrm{O}$ and stored at $4^{\circ} \mathrm{C}$ until use in infection studies.

\subsubsection{2 Primary and Challenge Infection Models}

L3 infectious stage larvae were harvested from faecal cultures and washed 3 times in $50 \mathrm{ml}$ sterile PBS. Larvae were re-suspended $200 \mathrm{~L} 3$ H. polygyrus/ $200 \mu \mathrm{l}$ doses and administered i.g. with a feeding syringe. Animals were left for 21 days then administered an anti-helminthic and 5 days later a second dose of anti-helminthic left 
for a further 10 days and challenged with 200 L3 H. polygyrus/ 200 $\mu$ l PBS i.g. At indicated times, animals were sacrificed by administration of lethal anaesthetic.

\subsubsection{3 Worm burdens, egg counts and fecundity}

Viable worm burdens were determined as for small intestines in section 2.8.2.1. 3 . Egg counts per caecum were determined by harvesting the entire caecum contents into

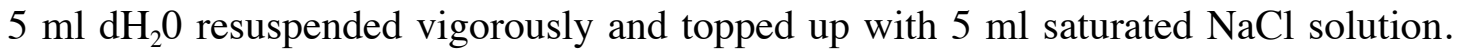
Upon vigorous resuspension a sample was removed and placed in the McMaster counting chamber where eggs were enumerated using the stereoscopic microscope. The total numbers of eggs per caecum were determined by multiplying the slide count by 400 and accounting for the dilution factor of the $150 \mu \mathrm{l}$ volume of the McMaster counting chamber that represents $10 \mathrm{ml}$ of the initial caecum solution. The fecundity was determined as total numbers of eggs per caecum divided by total numbers of viable female worms.

\subsubsection{Helminth models: Tissue preparation and processing.}

\subsubsection{1 Blood}

Blood was harvested from animals following lethal anaesthetic by cardiac puncture and $100 \mu \mathrm{l}$ blood was placed into $500 \mu \mathrm{l}$ Alsever's buffer, centrifuged at $250 \mathrm{~g}$ for 8 minutes, incubated with red cell lysis buffer at $37^{\circ} \mathrm{C}$ for 2 minutes, washed and resuspended in PBS. Live cells were identified by trypan blue exclusion dye (GIBCO, Invitrogen, Auckland, NZ) and counted using a haemocytometer and numbers of live cells per ml of blood was calculated.

\subsubsection{2 Lungs}

Lungs were harvested into IMDM, cut up finely and digested in $5 \mathrm{ml}$ Lung Digestion Buffer for 60 minutes at $37^{\circ} \mathrm{C}$. The digested lung was then passed through an 18 gauge needle and processed through $70 \mu \mathrm{M}$ cell strainer into single cell solution. The single cell solution was resuspended with MACS buffer and anti-mouse CD45 MACS Microbeads from Miltenyi Biotec (Pharmaco NZ Ltd, Auckland, NZ, 130-052-001) and incubated at $4^{\circ} \mathrm{C}$ for $15 \mathrm{~min}$. Washed in $4-5 \mathrm{ml}$ of MACs buffer, passed through 40 
$\mu \mathrm{M}$ cell strainer and separated on the autoMACS Pro Seperator (Miltenyi Biotec, Pharmaco NZ Ltd, Auckland, NZ). Live cells were identified by trypan blue exclusion dye (GIBCO, Invitrogen, Auckland, NZ) and counted using a haemocytometer.

\subsubsection{3 Lymph nodes}

Lymph nodes were harvested from infected animals into PBS, processed through 70 $\mu \mathrm{M}$ cell strainers into a single cell solution for analysis by flow cytometry. Live cells were identified by trypan blue exclusion dye (GIBCO, Invitrogen, Auckland, NZ) and counted using a haemocytometer.

\subsection{FACS analysis and staining}

Flow cytometry data were acquired on a BD LSRII SORP (Becton Dickinson, San Jose, CA) flow cytometer. Data were analyzed using FlowJo (TreeStar) software.

\subsubsection{Instrument Details: BD LSRII SORP (Becton Dickinson, San Jose, CA)}

The flow cell is manufactured by BD Biosciences. The external quartz cuvette surfaces are anti-reflection coated for maximum transmission of laser light. The sheath flow velocity is $17.5 \mathrm{ml} /$ minute provided by a gas pressured sheath flow pump, giving a sample flow velocity of $130 \mu 1 /$ minute at the maximum flow rate of 'hi' and the pressure dial turned 10 times to the highest possible setting, the sample flow pump is also gas pressured. The antibody and fluorochrome detectors of the BD LSRII SORP are tabulated in Table 2. 3. The laser specifications for the BD LSRII SORP are tabulated in Table 2. 4.

\subsubsection{Detection of surface marker expression}

Single cell suspensions were counted and washed at $400 \mathrm{~g} / 4$ minutes in FACS buffer and $2 \times 10^{6}$ cells were transferred into $5 \mathrm{ml}$ polystyrene round bottom test tubes. Cells were washed again and pellets resuspended vigorously, followed by incubating in FACS buffer containing $10 \mu \mathrm{g} / \mathrm{ml} 2.4 \mathrm{G} 2$ for 10 minutes on ice to block Fc receptors. Fluorescently conjugated antibodies against cell surface markers of interest were 
added to the $2.4 \mathrm{G} 2$ containing cell suspensions at the appropriate dilutions and incubated a further 30 minutes on ice. Cells were washed once with FACS buffer and then incubated with the appropriate streptavidin-conjugated fluorochrome for 10 minutes on ice, where required. Cells were washed a further two times and resuspended in $200 \mu \mathrm{l}$ FACS buffer, a $200 \mu \mathrm{l}$ DAPI stock solution was added directly prior to acquisition to obtain a final concentration of $40 \mathrm{ng} / \mathrm{ml}$ DAPI.

\subsubsection{Intracellular Staining}

Prior to staining for intracellular cytokines, IL-13, IL-5, IL-10 and IFN $\gamma$, cells were resuspended at $2 \times 10^{6}$ cells/ well in cIMDM and transferred into a 24 well plate. Cells were restimulated by incubating for 4 hours at $37^{\circ} \mathrm{C}$ on plate bound $\alpha \mathrm{CD} 3(10 \mu \mathrm{g} / \mathrm{ml})$ and soluble $\alpha \mathrm{CD} 28$ (1:50). GolgiStop ${ }^{\mathrm{TM}}$ (BD Pharmingen, Auckland, NZ. 554724) was also added to give a final concentration of $1 \mu \mathrm{g} / \mathrm{ml}$ and was present to prevent the export of proteins from the Golgi bodies. Cells were then harvested, washed in IMDM, resuspended in FACS buffer and stored on ice in preparation for labelling of intracellular markers. Intracellular staining for transcription factors GATA3 and BCL6 was performed on cells directly ex vivo.

For visualisation of live cells, $1 \mu$ l working solution of LIVEDEAD ${ }^{\circledR}$ Fixable Blue Dead Cell Stain in $1 \mathrm{ml}$ PBS was used to stain each sample for 30 minutes at $4^{\circ} \mathrm{C}$, samples were then washed in $2 \mathrm{ml}$ PBS whereby normal process for staining for surface markers was followed.

For visualisation of GFP post fixation, FIX \& PERM $^{\circledR}$ Fixation and Permeabilization kit (Invitrogen, Auckland, NZ. GAS-004) was utilised. The entire fixation and permeabilization was done at room temperature while protecting samples from the light. After staining to exclude dead cells and then for surface markers, cells were washed in $3 \mathrm{ml}$ FACS buffer at $400 \mathrm{~g} / 4$ minutes, resuspended and $100 \mu \mathrm{l}$ Fixation Medium (Reagent A) was added for 15 minutes. Cells were then washed in $3 \mathrm{ml}$ FACS buffer at $400 \mathrm{~g} / 4$ minutes, resuspended and $100 \mu \mathrm{l}$ Permeabilization Medium (Reagent B) containing fluorescently conjugated antibodies against intracellular markers of interest at the appropriate dilutions was added and incubated a further 30 
Chapter Two: Materials and Methods

minutes at room temperature. Cells were then washed and resuspended in $300 \mu l$ FACS buffer and stored on ice until acquisition.

\subsubsection{BD LSRII SORP set up and antibody staining controls}

To enable BD FACSDiva Software v6.1.1 to perform automatic compensation on data acquired on the BD LSRII SORP, either compensation beads as outlined in section 2.5. 3 and 2.5. 4 or naïve splenocytes from C57BL/6 mice were stained at appropriate concentration with antibodies and used as single stain controls. EtOH-killed splenocytes were stained with 4',6-Diamidino-2-phenylindole dihydrochloride (DAPI) for a single stain viability control while splenocytes from eGFP mice ${ }^{193}$ were used as single stains for the GFP positive control.

To ensure multi colour antibody panels were compensated correctly, fluorescence minus one (FMO) controls were used. FMO controls are the entire staining set minus one indicated fluorescent antibody. The FMO controls are used to confirm where to draw the gates and to confirm that auto-compensation is correct. If auto-compensation performed by BD FACSDiva Software v6.1.1 is incorrect, the data was manually compensated using the FlowJo (TreeStar) software.

The following are the hierarchical gating strategies used to analyse the indicated cell subsets. Each gating strategy is written parent followed by child with example flow cytometry plots attached as an appendix. The hierarchical gating strategy used to analyse the Th2 cells involved gating on the Doublet ${ }^{-}$Live cells (DAPI ${ }^{-}$), $\mathrm{CD}^{+}$, $\mathrm{CD}^{+}$, IL-4 Reporter $\mathrm{GFP}^{+}$(Figure A. 1). The hierarchical gating strategy used to analyse the allelic expression of IL-4 reporter in Th2 cells involved gating on Doublet Live cells (DAPI $)$, Lymphocyte gate, $\mathrm{CD}^{+}$, IL-4 Reporter $\mathrm{GFP}^{+}$versus IL-4 Reporter hCD2 ${ }^{+}$(Figure A. 2). The hierarchical gating strategy used to analyse the Tfh cells involved gating on the Doublet ${ }^{-}$, Live cells (DAPI ${ }^{-}$, Lymphocyte gate, CD4 ${ }^{+} \mathrm{B} 220^{-}$, $\mathrm{PD}^{+} \mathrm{CXCR}^{+}$(Figure A. 3). The hierarchical gating strategy used to analyse eosinphils involved gating on the Doublet, Live cells (DAPI $\left.{ }^{-}\right), \mathrm{SSC}^{\mathrm{hi}}, \mathrm{CD}^{-} 1 \mathrm{c}^{-}$, CD16/32+, CD49b (DX5) ${ }^{-}, \mathrm{CD}^{+} 1 \mathrm{~b}^{+}$, SiglecF $^{+}$(Figure A. 4). The hierarchical gating strategy used to analyse basophils involved gating on the Doublet, Live cells (DAPI'), non-B non-T cells (CD4CD19-), FceR1 $\alpha^{+}, \mathrm{CD} 49 \mathrm{~b}(\mathrm{DX} 5)^{+}, \mathrm{CD} 16 / 32^{+}, \mathrm{CD} 45^{\text {int }}$ (Figure A. 5). The hierarchical gating strategy used to analyse nuocytes involved gating on the 
Doublet, Lymphocyte gate, Live cells (DAPI'), CD45 ${ }^{+}$, Lineage $(\mathrm{CD} 3, \mathrm{~B} 220$, FceR1 $\alpha$, CD4, CD8, CD11c, GR1), Ly6A/E (Sca-1) ${ }^{+}$, CD49b (DX5)', CD117 (Ckit) ${ }^{+}$, CD44 (Figure A. 6).

\begin{tabular}{|l|llll|}
\hline \multicolumn{5}{|c|}{$\begin{array}{l}\text { Table 2. 3 Antibody and Fluorochrome Detectors of the BD LSRII SORP (Becton Dickinson, } \\
\text { San Jose, CA) }\end{array}$} \\
\hline LASER & DETECTOR NAME (TYPE) & LP MIRROR & BP FILTER TYPE & AMPLIFICATION \\
\hline $\mathbf{4 8 8} \mathbf{~ n m}$ & A (PMT) & 640 & $685 / 35$ & Log \\
& B (PMT) & 505 & $515 / 20$ & Log \\
& C (PMT) & None & $488 / 10$ & Linear \\
& FSC Photodiode & None & None & Linear \\
\hline $\mathbf{4 0 5} \mathbf{~ n m}$ & A (PMT) & 670 & $705 / 10$ & Log \\
& B (PMT) & 630 & $660 / 20$ & Log \\
& C (PMT) & 565 & $605 / 40$ & Log \\
& D (PMT) & 535 & $560 / 20$ & Log \\
& E (PMT) & 505 & $525 / 50$ & Log \\
& F (PMT) & None & $450 / 50$ & Log \\
\hline $\mathbf{3 5 5 n m}$ & A (PMT) & 635 & 670 LP & Log \\
& B (PMT) & None & $450 / 50$ & Log \\
\hline $\mathbf{6 3 8} \mathbf{n m}$ & A (PMT) & 740 & $780 / 60$ & Log \\
& B (PMT) & 685 & $710 / 50$ & Log \\
& C (PMT) & None & $670 / 14$ & Log \\
\hline $\mathbf{5 3 2} \mathbf{~ n m}$ & A (PMT) & 735 & $780 / 60$ & Log \\
& B (PMT) & 685 & $695 / 40$ & Log \\
& C (PMT) & 600 & $660 / 40$ & Log \\
& D (PMT) & 600 & $610 / 20$ & Log \\
& E (PMT) & None & $575 / 26$ & Log \\
\hline & & & & \\
\hline
\end{tabular}


Chapter Two: Materials and Methods

Table 2. 4 Laser Specifications for the BD LSRII SORP (Becton Dickinson, San Jose, CA)

\begin{tabular}{|c|c|c|c|c|c|}
\hline Laser Line & UV & Violet & Blue & Green & Red \\
\hline Wavelength & $355 \mathrm{~nm}$ & $405 \mathrm{~nm}$ & $488 \mathrm{~nm}$ & $532 \mathrm{~nm}$ & $640 \mathrm{~nm}$ \\
\hline Power & $20 \mathrm{~mW}$ & $50 \mathrm{~mW}$ & $100 \mathrm{~mW}$ & $150 \mathrm{~mW}$ & $40 \mathrm{~mW}$ \\
\hline Polarization & $\begin{array}{l}>100: 1 \\
\text { horizontal }\end{array}$ & $>100: 1$ vertical & $>100: 1$ vertical & $>100: 1$ vertical & $\begin{array}{l}>100: 1 \\
\text { vertical }\end{array}$ \\
\hline $\begin{array}{l}\text { Beam } \\
\text { Diameter at } \\
1 / e^{2}\end{array}$ & $0.9 \pm 0.2 \mathrm{~mm}$ & $1.4 \mathrm{~mm}$ & $\begin{array}{l}0.70 \pm 0.05 \\
\mathrm{~mm}\end{array}$ & $\begin{array}{l}0.34 \pm 0.02 \\
\mathrm{~mm}\end{array}$ & $1 \mathrm{~mm}$ \\
\hline $\begin{array}{l}\text { Beam } \\
\text { Divergence }\end{array}$ & $< \pm 8 \mathrm{mrad}$ & $0.4 \mathrm{mrad}$ & $<1.2 \mathrm{mrad}$ & $2.2 \mathrm{mrad}$ & $1 \mathrm{mrad}$ \\
\hline Manufacturer & $\begin{array}{l}\text { Lightwave }{ }^{\circledR} \\
\text { Electronics }\end{array}$ & Coherent & Coherent & Coherent & Coherent \\
\hline Model Name & $\begin{array}{l}\text { Xcyte Quasi- } \\
\text { Continuous } \\
\text { Wave Laser }\end{array}$ & $\begin{array}{l}\text { CUBE Diode } \\
\text { Laser } 405-50 C\end{array}$ & $\begin{array}{l}\text { Sapphire 488- } \\
100 \text { Optically } \\
\text { Pumped } \\
\text { Semiconductor } \\
\text { Laser }\end{array}$ & $\begin{array}{l}\text { Compass } \\
315 \mathrm{M}-150 \\
\text { Continuous } \\
\text { Wave Diode } \\
\text { Pumped Laser }\end{array}$ & $\begin{array}{lr}\text { CUBE } & \text { Diode } \\
\text { Laser } & 640- \\
40 \mathrm{C} & \end{array}$ \\
\hline Part Number & $\begin{array}{l}\text { CY-355-020- } \\
6224\end{array}$ & 1174298 & 1055516 & 1100419 & 1069417 \\
\hline $\begin{array}{l}\text { Amplitude } \\
\text { Noise }\end{array}$ & $\begin{array}{l}<1 \% \mathrm{rms} \\
(10 \mathrm{~Hz} \text { to } 1 \\
\mathrm{MHz})\end{array}$ & $\begin{array}{l}<1 \% \mathrm{rms} \\
(10 \mathrm{MHz} \text { to } 0.5 \\
\mathrm{GHz})\end{array}$ & $\begin{array}{l}<0.25 \% \mathrm{rms} \\
(20 \mathrm{~Hz} \text { to } 2 \\
\mathrm{MHz})\end{array}$ & $\begin{array}{l}<0.25 \% \mathrm{rms} \\
(10 \mathrm{~Hz} \text { to } 1 \\
\mathrm{GHz})\end{array}$ & $\begin{array}{l}<0.1 \% \mathrm{rms} \\
(20 \mathrm{Hzs} \text { to } 10 \\
\mathrm{MHz})\end{array}$ \\
\hline $\begin{array}{l}\text { Repetition } \\
\text { Rate }\end{array}$ & $100 \pm 10 \mathrm{MHz}$ & $\mathrm{N} / \mathrm{A}$ & $\mathrm{N} / \mathrm{A}$ & $\mathrm{N} / \mathrm{A}$ & $\mathrm{N} / \mathrm{A}$ \\
\hline Pulse Width & $>10 \mathrm{ps}$ & $\mathrm{N} / \mathrm{A}$ & $\mathrm{N} / \mathrm{A}$ & $\mathrm{N} / \mathrm{A}$ & $\mathrm{N} / \mathrm{A}$ \\
\hline $\begin{array}{l}\text { Power } \\
\text { Stability } \\
\text { (8 hours) }\end{array}$ & $< \pm 1 \%$ & $< \pm 2 \%$ & $<2 \%$ & $< \pm 2 \%$ & $< \pm 2 \%$ \\
\hline $\begin{array}{l}\text { Pointing } \\
\text { Stability }\end{array}$ & $<20 \mu \mathrm{rad} /{ }^{\circ} \mathrm{C}$ & $<6 \mu \mathrm{rad} /{ }^{\circ} \mathrm{C}$ & $<30 \mu \mathrm{rad} /{ }^{\circ} \mathrm{C}$ & $<6 \mu \mathrm{rad} /{ }^{\circ} \mathrm{C}$ & $<6 \mu \mathrm{rad} /{ }^{\circ} \mathrm{C}$ \\
\hline Beam Quality & $\mathrm{M}^{2}<1.2$ & $\mathrm{M}^{2}<1.3$ & $\mathrm{M}^{2}<1.1$ & $\mathrm{M}^{2}<1.3$ & $\mathrm{M}^{2}<1.3$ \\
\hline $\begin{array}{l}\text { Spatial Mode } \\
\text { (Far field) }\end{array}$ & TEM $_{00}$ & TEM $_{00}$ & TEM $_{00}$ & TEM $_{00}$ & TEM $_{00}$ \\
\hline
\end{tabular}




\subsection{Histology}

The Department of Pathology and Molecular Medicine, University of Otago, Wellington prepared and stained all histology sections. Tissue samples were fixed in $10 \%$ formalin and embedded in paraffin. Samples were cut into $4 \mu \mathrm{m}$ sections and stained with Mayer's Haematoxylin and Periodic Acid Schiffs reagent (PAS). All bronchioles with a diameter of between 200 to $600 \mu \mathrm{m}$ were counted and the number of PAS-positive cells per bronchiole was reported.

\subsection{Enzyme-linked Immunosorbent assay (ELISA)}

Nunc Maxisorp plates 96-well plates (Thermo Fisher Scientific, Auckland, NZ. 430341) were coated with $100 \mu \mathrm{l} /$ well of $6 \mathrm{HD} 5(5 \mathrm{mg} / \mathrm{ml})$ for detection of $\operatorname{IgE}$ or 11B11 $(2 \mu \mathrm{g} / \mathrm{ml})$ for detection of IL-4 diluted in carbonate coating buffer and incubated for 2 hours at $37^{\circ} \mathrm{C}$ or overnight at $4^{\circ} \mathrm{C}$. Plates were washed by submerging in a bucket containing 4 litres of PBS and $0.05 \%$ Tween-20 then blocked with $200 \mu \mathrm{l} /$ well of $10 \%$ FCS in PBS at 2 hours for $37^{\circ} \mathrm{C}$. Samples were serial diluted at 1:10, 1:100, 1:1000, 1:10000 and added $100 \mu \mathrm{l} /$ well. The standard was serial diluted with a starting concentration of $100 \mathrm{ng} / \mathrm{ml}$. Plates were incubated for 2 hours at $37^{\circ} \mathrm{C}$. Plates were washed and secondary antibody R1E4-biotin ( $3 \mu \mathrm{g} / \mathrm{ml})$ or IL-4-biotin $(2 \mu \mathrm{g} / \mathrm{ml})$ in $10 \%$ FCS/ PBS was added $100 \mu \mathrm{l} /$ well and incubated for 2 hours at $37^{\circ} \mathrm{C}$. Plates were washed and 1 times solution HRP conjugate diluted in 10\% FCS/ PBS was added at $100 \mu \mathrm{l} /$ well for 1 hour at $37^{\circ} \mathrm{C}$. Plates were washed and developed with 100 $\mu \mathrm{l} /$ well TMB substrate A:B (1:1) and stopped with $100 \mu \mathrm{l} /$ well $1 \mathrm{M} \mathrm{H}_{2} \mathrm{SO}_{4}$. Absorbance was read at $450 \mathrm{~nm}$ on the Versamax Microplate Spectrophotometer (Molecular Devices, Germany). 


\subsection{Multiplex Cytokine Detection}

Single cell suspensions MACS sorted for $\mathrm{CD} 45^{+}$cells from lungs or lymph nodes of naïve or infected mice were and re-stimulated for 24 hours at $37^{\circ} \mathrm{C}$ on Dynabeads ${ }^{\circledR}$ Mouse T-Activator CD3/ CD28 (Invitrogen, Auckland, NZ. 114-56D) and rIL-2 (100 $\mathrm{U} / \mathrm{ml}$ ). Supernatant was stored at $-20^{\circ} \mathrm{C}$ until further analysis. Levels of IL-4, IL-13, IL-5, IL-9, IL-17 and IFN $\gamma$ were determined according to the manufacturer's instructions using the Bio-Plex Pro ${ }^{\mathrm{TM}}$ Mouse Cytokine 23-plex Assay (Bio-Rad, Auckland, NZ. M60-009RDPD). Briefly, a mixture of capture beads at the correct concentration specific for each cytokine was prepared in bioplex assay buffer and the plate was washed twice with bioplex assay buffer by adding $200 \mu \mathrm{l}$ per well and removing by vacuum manifold (Bio-Rad, USA). Supernatant samples were diluted at 1:2 in bioplex assay buffer and $50 \mu \mathrm{l}$ was added to the wells. The plate was then incubated with agitation for 2 hours at room temperature and then washed twice as described above. Biotinylated detection antibodies were made up at a 1:30 dilution and $25 \mu \mathrm{l}$ was added to the plate followed by a 1 hour incubation with agitation at room temperature. The plate was then washed twice and incubated with $100 \mu \mathrm{l}$ of Streptavidin-PE for 30 minutes at room temperature. The plate was washed three times as described above and samples were then resuspended in $200 \mu$ l of bioplex assay buffer and analysed using the Bio-Plex ${ }^{\mathrm{TM}}$ system. Cytokine concentrations were determined against commercial standards (Bio-Rad, Auckland, NZ) and calculated using the provided software (Bio-Plex manager software, Bio-Rad, USA).

\subsection{ELISpot}

IL-4 production by CD4 $\mathrm{T}$ cells was analysed according to the manufacturer's instructions using Mouse IL-4 ELISpot assay (Mabtech, Auckland, NZ. 3311-2H). Briefly, ELISpot plates were coated with 11B11 (15 $\mu \mathrm{g} / \mathrm{ml})$ in sterile PBS. Washed 5 times in $200 \mu \mathrm{l}$ sterile water/ well and removed by vacuum manifold (Bio-Rad, USA). Incubated with $100 \mu \mathrm{l}$ antibody solution overnight at $4^{\circ} \mathrm{C}$ and washed 5 times in 200 $\mu 1$ sterile PBS/ well. Wells are incubated for minimum of 30 minutes at room temperature with $200 \mu \mathrm{l} /$ well of $10 \%$ FCS cIMDM. Cell suspensions in titration were re-stimulated for 18 hours at $37^{\circ} \mathrm{C}$ with $5 \% \mathrm{CO}_{2}$ on Dynabeads ${ }^{\circledR}$ Mouse T-Activator CD3/ CD28 (Invitrogen, Auckland, NZ. 114-56D) and rIL-2 (100 U/ ml). Plates were 
washed in PBS and incubated at room temperature for 2 hours with $100 \mu \mathrm{l} /$ well of $0.5 \%$ FCS in PBS containing $1 \mu \mathrm{g} / \mathrm{ml}$ detection antibody (BVD6-24G2-biotin). Plates were washed in PBS and incubated at room temperature for 1 hour with $100 \mu \mathrm{l} /$ well of Streptavidin-HRP in PBS-0.5\% FCS. Plates were washed and $100 \mu \mathrm{l} /$ well of substrate solution (1:1) was added to allow distinct spots to develop. Plates were washed extensively in tap water, allowed to dry and spots were counted using an ELISpot reader.

\section{9 Statistics}

Statistical calculations were performed using the Graphpad Prism ${ }^{\circledR}$ Version 5.0c statistical package (Graphpad Software Inc., San Diego, CA, USA). The type of statistical test used is described in the appropriate figure legends. 

Chapter Three:

Investigating the potential polarising role of IL-25 during the differentiation of Th2 cells. 
Chapter Three: Investigating the potential role of IL-25 during the differentiation of Th2 cells

\subsection{Background}

The differentiation of a naïve CD4 $\mathrm{T}$ cell into a $\mathrm{T}$ helper 2 cell requires TCR engagement of cognate antigen presented on MHC class II complex, co-stimulation and a polarising cytokine environment. Seminal studies into polarising cytokines required for the differentiation of naïve CD4 T cells into Th2 cells, illustrated that IL4 was required to secure its own production ${ }^{8}$. In contrast more recent studies utilising protein reporter technology have shown Th2 cells are able to differentiate and disperse in vivo without the need for IL-4 signalling ${ }^{65}$, indicating IL-4 is not the Th2 cell polarising cytokine. As this polarising cytokine is required at the same time as the antigen is presented to the naïve CD4 T cells, the cell producing it is quite probably part of the innate immune system and would be detectable in the lymph node $\mathrm{T}$ cell zone.

The results of multiple studies have indirectly suggested that the innate cytokine IL-25 has a role in Th2 cell differentiation. For example, the over-expression of IL-25 or local administration of the recombinant protein have been described as driving $\mathrm{Th} 2$ "like" phenotypes; indicating a potential role for IL-25 in T helper type 2 immunity 136,138,196. Secondly, the mRNA for IL-25 receptor was detected in both naïve CD4 T cells and in differentiated Th2 cells; indicating naïve CD4 T cells could potentially respond to signalling by IL-25 ${ }^{81}$. Thirdly, the addition of rIL-25 to naïve CD4 T cells undergoing polyclonal activation resulted in the production of IL-4; indicating IL-25 could potentially act as a polarising factor in the differentiation of Th2 cells ${ }^{81,197}$. The experimental models and methods of these experiments however, created difficulty discerning whether rIL-25 was acting as a cell proliferation factor or as a Th2 cell polarising factor. These results also differed from data published by Wang et al. that described rIL-25 as a factor involved in expanding memory Th2 cells, but unable to act directly on naïve CD4 T cells ${ }^{147}$. Therefore, this chapter set out to clarify these conflicting results and investigate whether IL-25 was required for the differentiation of Th2 cells.

A confounding factor to investigating the differentiation of $\mathrm{Th} 2$ cells is the manner in which these cells become IL-4 producers, whereby the production by the early- 
activated CD4 $\mathrm{T}$ cell results in IL-4 production that sustains and enhances its own production in an autocrine manner ${ }^{84}$. As there is an IL-4 autocrine feedback loop the differentiation of Th2 cells must be investigated in environments which are wholly deficient in IL-4 signalling and hence enables the potential polarising signal of IL-25 to be teased out. Unlike previous investigations into the role of IL-25 in Th2 differentiation, this chapter used a previously described IL-4 GFP reporter mouse model (G4 model) ${ }^{119}$ that allows in situ monitoring of IL-4 production on a cell-tocell basis. This model has a minimal requirement for manipulation, bypassing the need for restimulation, fixation or intracellular staining. The G4 reporter mouse has GFP inserted into the first exon of the il4 allele. This insertion results in the production of GFP protein instead of IL-4. The G4 reporter protein has been shown to faithfully report the expression of IL-4 protein ${ }^{119}$. Animals homozygous for the G4 insertion are IL-4 deficient and are referred to as $\mathrm{G}^{+/+}$mice. Heterozygous $\mathrm{G} 4^{+/-}$ animals retain intact IL-4 signalling pathways. To create an IL-4 deficient antigen specific CD4 T cell, a TCR transgenic (Tg) OTII mouse was crossed with the $4^{+/+}$ mouse and the F1 litter backcrossed to obtain $\mathrm{G}^{+/+}{ }^{-+} \mathrm{OTII}^{+/+}$strain ${ }^{195}$. This mouse was used to design an in vitro culture system in order to investigate the role of IL-25 on naïve CD4 T cells in the absence of IL-4 signalling. The G4 reporter mice were also crossed with IL-25 deficient animals ${ }^{194}$, to enable the investigation of in vivo Th2 differentiation in the absence of IL-25 with or without IL-4 signalling. This reporter system enables one to capture the in vivo generated $\mathrm{Th} 2$ phenotype and avoids any in vitro re-activation, which may confound results. Utilising these novel reporter systems the intent of this chapter was to investigate whether IL-25 was indeed a polarising factor involved in the differentiation of Th2 cells. 
Chapter Three: Investigating the potential role of IL-25 during the differentiation of Th2 cells

\subsection{Research Objectives}

The research objectives of this chapter were to develop novel in vitro and in vivo Th2 cell differentiation models and use them to investigate whether IL-25 was required for the IL-4 independent pathway of Th2 cell differentiation. It was hypothesised that an in vitro IL-4 independent Th2 cell differentiation system could provide a useful tool for dissecting the signalling requirements for Th2 cell differentiation and enable the investigation into whether the innate cytokine IL-25 could in fact be a Th2 polarising cytokine. The in vivo Th2 cell differentiation assay optimised an allergen Th2 model of differentiation utilising a single draining lymph node to ensure the earliest stages of Th2 cell differentiation could be captured and thereby enabling an investigation into whether the innate cytokine IL-25 plays a polarising role during in vivo Th2 cell differentiation.

Summarised aims:

- Establish a simplified in vitro assay that allows us to isolate the core components of early IL-4 independent Th2 differentiation and investigate whether IL-25 is a polarising cytokine involved in this differentiation.

- Establish an in vivo assay that allows us to investigate the role of endogenous IL-25 in the differentiation of a Th2 cell. 


\subsection{Results}

\subsection{Optimisation of the in vitro assay for IL-4 independent differentiation of Th2 cells: Polyclonal differentiation.}

The purpose of these studies was to investigate whether early Th2 differentiation in vitro could occur in the absence of IL-4. A differentiated Th2 cell was defined as a cell that produces IL-4, the hallmark Th2 cytokine. Cultures were set up with titrations of naïve $\mathrm{G} 4{ }^{+/+} \mathrm{CD} 4 \mathrm{~T}$ cells on plate bound $\alpha \mathrm{CD} 3$ and soluble $\alpha \mathrm{CD} 28$ in the presence of rIL-2, with or without rIL-4 to monitor the role of IL-4 in Th2 cell development. Stimulation with $\alpha \mathrm{CD} 3 / \alpha C D 28$ is used to simulate the APCs activation of the $\mathrm{T}$ lymphocytes TCR through antigen-MHC complex and co-stimulation through CD80/CD86 binding CD28. Naïve $\mathrm{G} 4^{+/+}$CD4 T cells were monitored over time for the expression of GFP in the presence or absence of rIL-4. While IL-4 was required for optimal Th2 cell differentiation there was a small yet persistent Th2 cell population present even in absence of IL-4 (Figure 3. 1 A).

To confirm the GFP positive cells were accurately indicating IL-4 induction, wild type $\mathrm{IL}-4^{+/+} \mathrm{CD} 4 \mathrm{~T}$ cells that contained no GFP reporter inserts were included to control for autofluorescence (Figure 3.1 B) and $\mathrm{G}^{+/+}{ }^{-+} \mathrm{STAT} 6^{-/}$cells were included to ensure no contaminating IL-4 was present. It was noted that in the absence of IL-4, similar levels of Th2 differentiation occurred in the $\mathrm{G}^{+/+}$and the $\mathrm{G}^{+/+}{ }^{-\mathrm{STAT}} 6^{-/}$cells (Figure 3. 1 B). Moreover, when rIL-4 was added to the culture the $\mathrm{G}^{+/+}$cells showed increased frequency of GFP expression as expected, but the $\mathrm{G} 4^{+/+} \mathrm{STAT}^{-/-}$cells maintained similar frequencies of GFP producing cells as the IL-4 deficient cultures as these cells are unable to respond to IL-4. This data confirms the small population of approximately $1 \%$ of the CD4 T cells that were $\mathrm{GFP}^{+}$observed in (Figure 3.1 A Right Panel) were indeed IL-4 independent Th2 differentiated cells. 
Chapter Three: Investigating the potential role of IL-25 during the differentiation of $\underline{\text { Th2 cells }}$

A

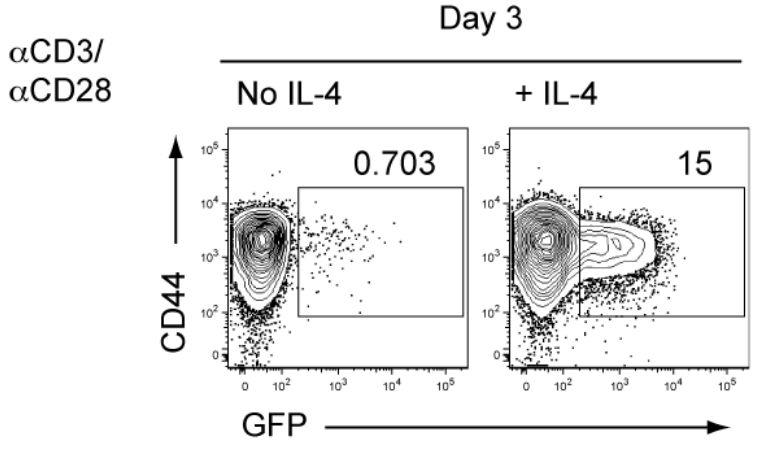

B $\alpha \mathrm{CD} 3 /$
$\alpha \mathrm{CD} 28$

$$
1
$$$$
\mathrm{IL}-4^{+/+}
$$$$
\text { CD4 T cells }
$$

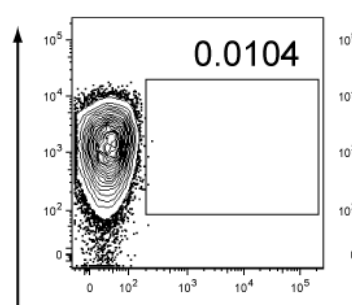

$\mathrm{G}^{+/+}$ CD4 T cells

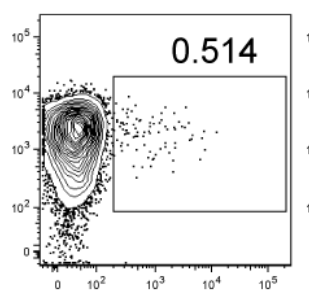

$\mathrm{G}^{+/+}{ }^{+}$Stat6 ${ }^{-/-}$

CD4 T cells

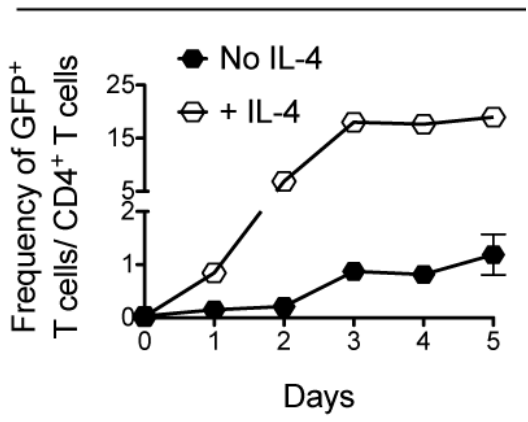

Figure 3. 1 Polyclonal activation of CD4 T cells differentiates Th2 cells even in the absence of IL-4. Positively selected naïve CD4 T cells from spleens were incubated on plate bound $\alpha C D 3(10 \mu \mathrm{g} / \mathrm{ml})$, soluble $\alpha \mathrm{CD} 28(1: 50)$ and rIL-2 $(50 \mathrm{U} / \mathrm{ml})$ with or without rIL-4 $(1000 \mathrm{U} / \mathrm{ml})$ for up to 5 days. Cells were stained and analysed by flow cytometry for the presence of GFP ${ }^{+}$ Th2 cells and A Right Panel: Representative FACS plots at day 3 A Left Panel: enumerated over 5 days in culture. B: Day 3 activated cells from IL-4 $4^{+/+}$reporter negative, $\mathrm{G} 4^{+/+}$reporter positive IL-4 deficient and $\mathrm{G}^{+/+}{ }^{\text {STAT }} 6^{-/-}$reporter positive STAT6 deficient mice were analysed on by flow cytometry for the production of Th2 cells as reported by GFP. Data was compiled from one experiment with triplicate samples. 
Chapter Three: Investigating the potential polarising role of IL-25 during the differentiation of Th2 cells.

\subsection{Optimisation of the in vitro assay for IL-4 independent differentiation of Th2 cells: Titrating activation stimulus.}

Th2 cell differentiation is favoured by low antigen concentrations or weak binding of the TCR complex by MHC II ${ }^{33,35,198,199}$. Hence, the strength of engagement of the TCR was titrated out by reducing the concentration of plate bound $\alpha \mathrm{CD} 3$. The lowest concentration of $\alpha \mathrm{CD} 3$ that still maintained cell proliferation and survival was determined to be $0.05 \mu \mathrm{g} / \mathrm{ml}$ of plate-bound $\alpha \mathrm{CD} 3$ (Figure 3. $2 \mathrm{~B}$ ). For the entire CD4 $\mathrm{T}$ cell population to obtain an activated phenotype, measured by expression levels of the activation marker $\mathrm{CD} 44,1 \mu \mathrm{g} / \mathrm{ml} \alpha \mathrm{CD} 3$ was required (Figure 3. 2 A). This concentration was more effective at differentiating a greater frequency of Th2 cells than the higher concentration of $10 \mu \mathrm{g} / \mathrm{ml} \alpha \mathrm{CD} 3$. Thereby supporting the theory that lower activation stimulus is optimal for the initiation of Th2 cells.

When comparing primary and secondary lymphoid organs as sources of Th2 cells it appears that CD4 $\mathrm{T}$ cells from the thymus far more readily differentiated into GFP producing cells than the CD4 T cells from the spleen (Figure 3.2 A). iNKT cells are found in abundance in the thymus of younger mice and readily produce IL- 4 cytokine when stimulated ${ }^{200}$. These investigations suggest that the aforementioned polyclonally activated $\mathrm{GFP}^{+} \mathrm{CD} 4 \mathrm{~T}$ cells could in fact be iNKT cells. To rule out this possibility it was decided to develop an antigen specific activation system using OTII transgenic mice. These mice do not respond to $\alpha$-Galactosylceramide, the potent activator of iNKT cells, inferring that these animals lack iNKT cells (personal communication Franca Ronchese. MIMR, NZ). 
Chapter Three: Investigating the potential role of IL-25 during the differentiation of $\underline{\text { Th2 cells }}$

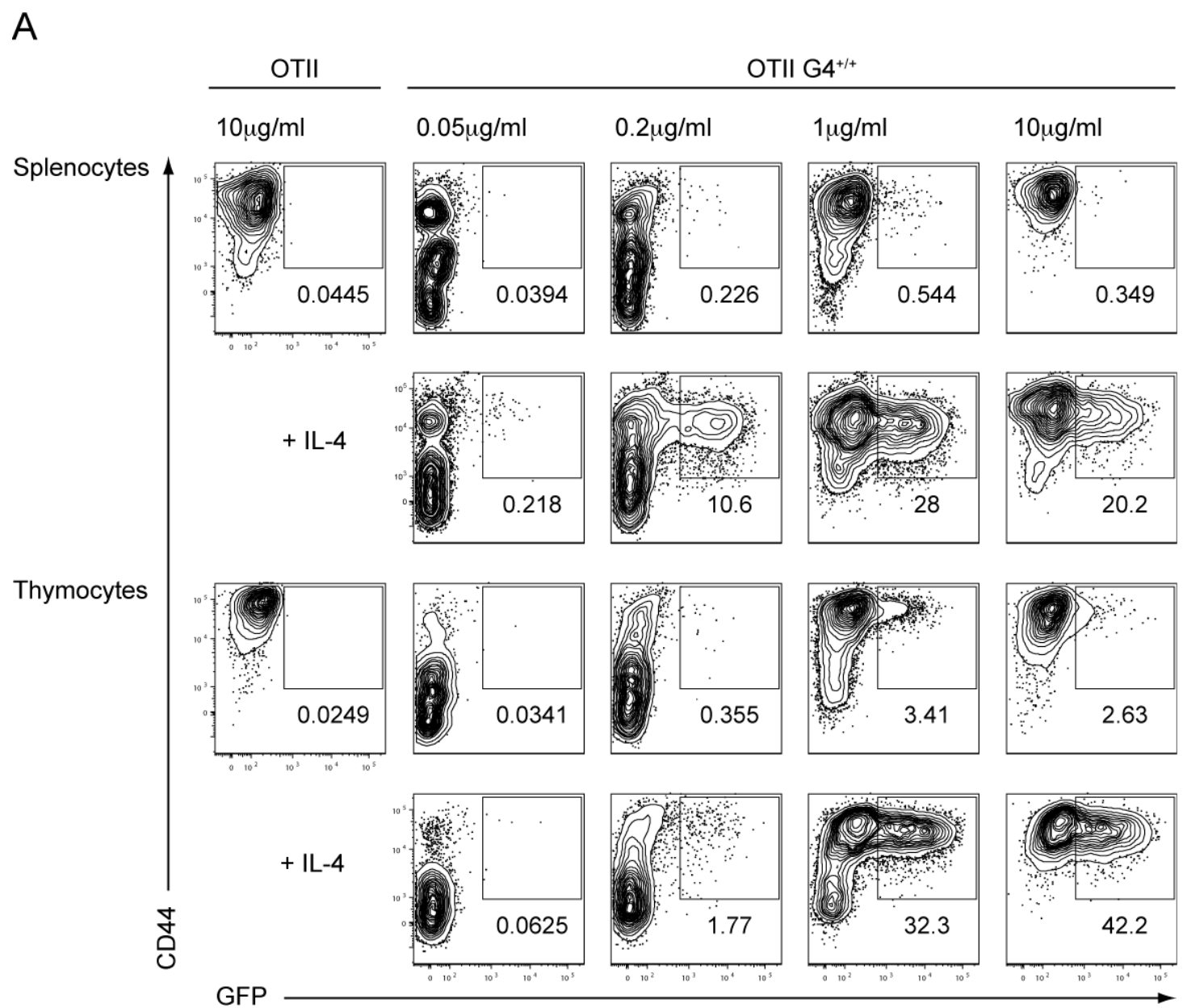

B
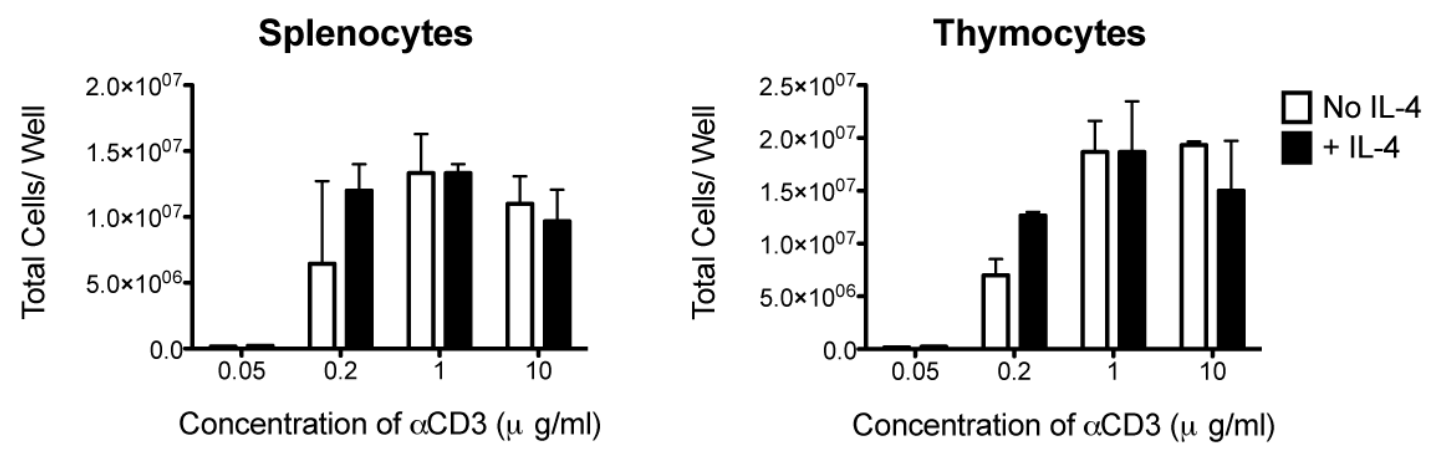

Figure 3. 2 Polyclonal activation of CD4 T cells requires sufficient concentrations of $\alpha$ CD3 for proliferation and Th2 differentiation. Positively selected $1 \times 10^{6}$ naïve CD4 T cells from spleens or thymi were incubated for 3 days on plate bound $\alpha C D 3$ at various indicated concentrations along with soluble $\alpha$ CD28 (1:50) and rlL-2 $(50 \mathrm{U} / \mathrm{ml})$ with or without rlL-4 $(1000 \mathrm{U} / \mathrm{ml})$ A: Activated cells from reporter negative TCR Tg (OTII) and reporter positive IL-4 deficient TCR Tg (OTII G4 ${ }^{+/+}$) were analysed by flow cytometry for the induction of Th2 cells as reported by GFP. B: Total viable cells were determined using trypan blue exclusion dye. Data was compiled from one experiment with triplicate samples. Bar graphs show mean \pm SEM. 


\subsection{Optimisation of the in vitro assay for IL-4 independent differentiation of Th2} cells: TCR expression in transgenic mice does not alter Th2 responses to polyclonal activation.

An alternative strategy to specifically activate CD4 T cells in vitro was employed; involving antigen-presenting cells, antigen and TCR restricted antigen specific T

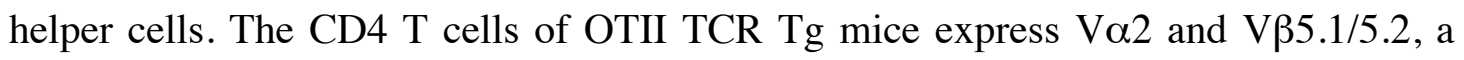
TCR complex specific for the OVA peptide ISQAVHAAHAEINEAGR (ISQ peptide) 195. These mice are on a C57BL/6 background and so were crossed with GFP reporter mice on the same background thereby generating OTII G4 $4^{+/+}$double Tg mice, which have a high frequency of OVA specific G4 reporter T cells. To confirm the cells functional abilities were not damaged by the TCR restriction, they were subjected to polyclonal activation alongside wild type reporter T cells and monitored for Th2 cell differentiation. The OTII G4 ${ }^{+/+} \mathrm{T}$ cells had a lower frequency of Th2 differentiation but a higher proliferation rate in the absence of IL-4 (Figure 3. 3 A\&B), inferring the insertion of the transgenic TCR will not result in non-specific overproduction of GFP by the TCR Tg CD4 T cells.

\subsection{Optimisation of the in vitro assay for IL-4 independent differentiation of Th2} cells: Confirming TCR expression in transgenic mice.

To investigate the efficiency of the OTII transgene expression in OTII $\mathrm{G}^{+/+}$mouse strain, the frequency of double positive $V \beta 5.1 / 5.2$ and $V \alpha 2$ chains on naïve blood CD4 T cells was determined. Strains of TCR Tg mice are known to have different levels of efficiency in expression of the transgenic $\alpha$ and $\beta$ TCR chains ${ }^{201}$. The average frequency of dual expression of the transgenic $\alpha$ and $\beta$ TCR chains was usually around $92.51( \pm 3.398) \%$ per litter of animals tested (Figure 3. 4). This meant the majority of CD4 T cells could respond to ISQ peptide upon presentation. 
Chapter Three: Investigating the potential role of IL-25 during the differentiation of Th2 cells

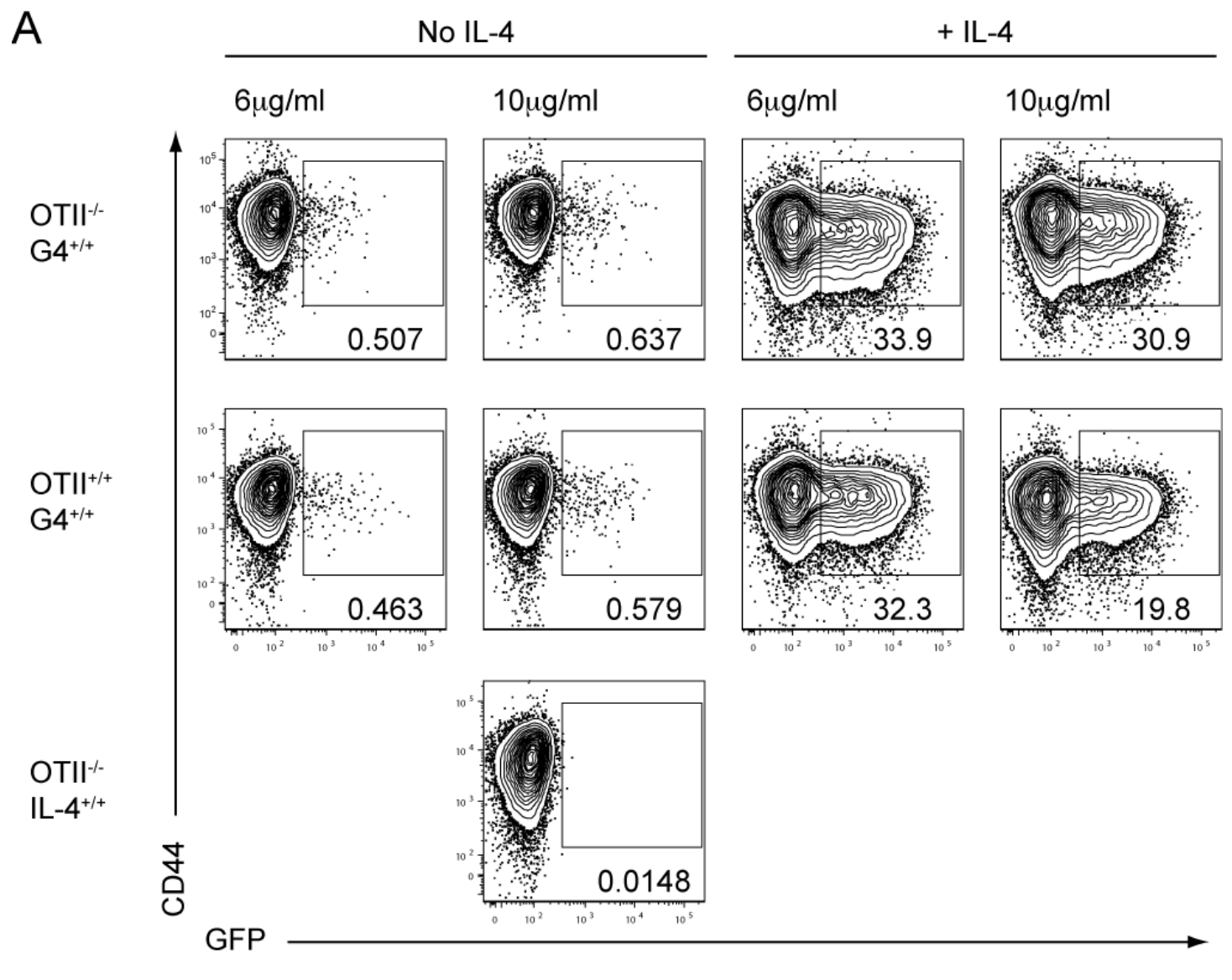

B

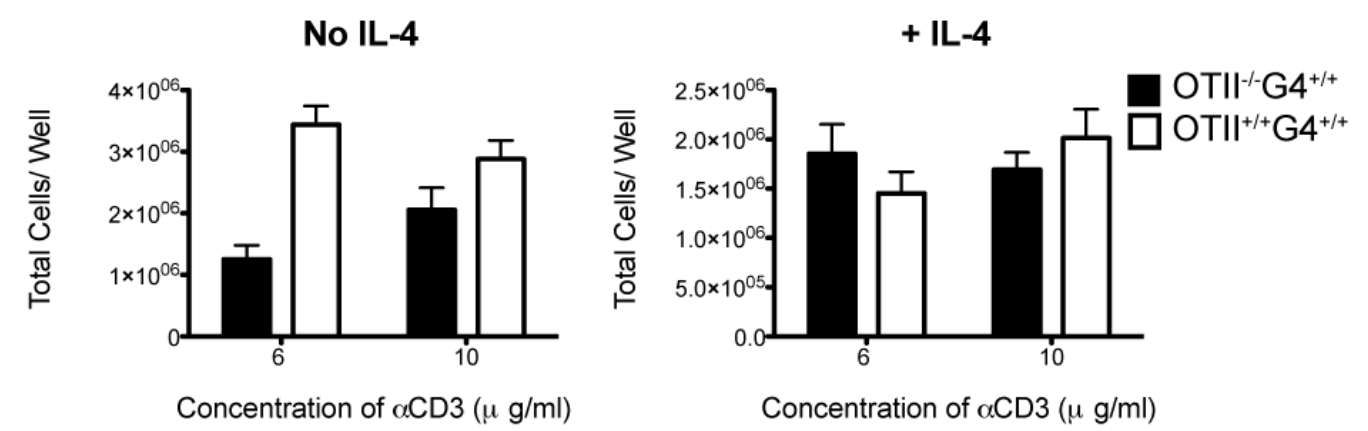

Figure 3. 3 Polyclonal activation of TCR Tg OTII G4 ${ }^{+/+} \mathrm{CD} 4$ T cells did not result in nonspecific GFP production. Positively selected naïv CD4 T cells from spleens were incubated on plate bound $\alpha \mathrm{CD} 3$ at indicated concentrations, soluble $\alpha \mathrm{CD} 28(1: 50)$ and rlL-2 $(50 \mathrm{U} / \mathrm{ml})$ with or without rlL-4 $(1000 \mathrm{U} / \mathrm{ml})$ for 3 days. A: Cells from reporter negative TCR Tg $\left(\mathrm{OTII}^{+/+} \mathrm{GL}^{-/ /}\right)$and reporter positive IL-4 deficient TCR Tg $\left(\mathrm{OTII}^{+/+} \mathrm{GL}^{+/+}\right)$mice and wild type (OTII $\left.{ }^{\prime} \mathrm{IL}-4^{+/+}\right)$were stained and analysed by flow cytometry for the presence of Th2 cells. $\underline{\mathrm{B}}$ : Total cell counts per well in the presence or absence of rIL-4. Data was compiled from one experiment with triplicate samples. Bar graphs show mean \pm SEM. 
A

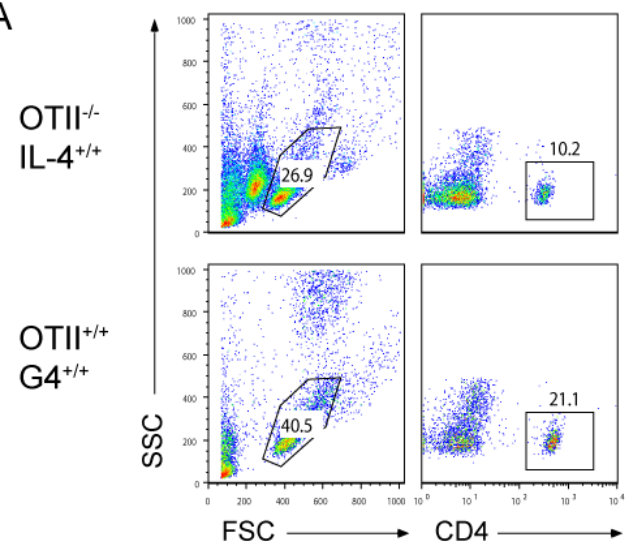

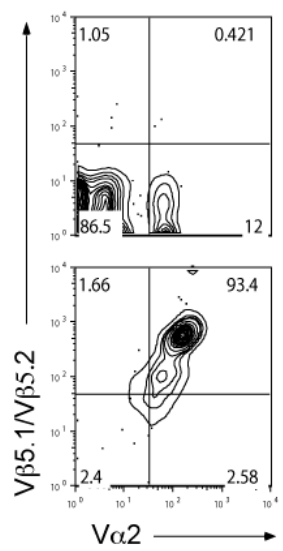

B

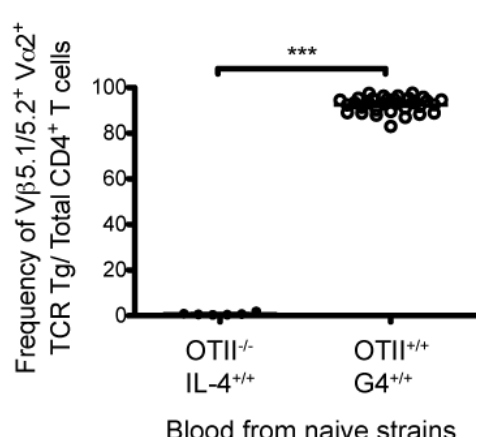

Figure 3. 4 The majority of CD4 T cells in TCR Tg OTII G4 $4^{+/+}$mice express both transgenic $\alpha$ and $\beta$ chains. Blood from naïve mice was processed and analysed by flow cytometry for $V \alpha 2$ and $\mathrm{V} \beta 5.1 / 5.2$ expression. A: Flow plots depicting hierarchical gating strategy from OTII ${ }^{-1 / L-}$ $4^{+/+}$wild type controls (top row) and TCR Tg OTII G4 ${ }^{+/+}$reporter mice (bottom row) $\underline{\text { B: }}$ Graphical representation of flow data frequency of dual expression of transgenic TCR chains per total CD4 T cells. Mann-Whitney Test $p<0.0001 ; n \geq 6$ mice per/group all OTII mice were tested.

\subsection{Optimisation of the in vitro assay for IL-4 independent differentiation of Th2} cells: APC to T cell ratio for maximal Th2 cell differentiation.

An antigen specific in vitro culture system requires the presence of an antigenpresenting cell (APC) such as bone marrow derived dendritic cells, which can present antigen on the MHC class II molecule to CD4 T cells. These cells not only present antigen but also provide co-stimulatory signals to the $\mathrm{T}$ cell. The optimal ratio of APCs to $\mathrm{T}$ cells would result in maximal $\mathrm{Th} 2$ differentiation and sufficient cell numbers for post culture analysis. A titrated ratio of 1:4, 1:16 and 1:64 bone marrow derived dendritic cell (BMDC): T cell ratio was compared over the 3-day activation period. LPS matured rGMCSF + rIL-4 differentiated BMDCs were pulsed with ISQ peptide to load the MHC class II molecules then cultured at the indicated concentrations with the OTII G4 ${ }^{+/+}$T cells in rIL-2 (100 U/ml) with or without rIL-4 (1000 U/ml). The Th2 cell frequencies remained low but similar throughout the BMDC: $\mathrm{T}$ cell ratios in IL-4 deficient conditions. However the 1:4 BMDC to T cell ratio was optimal for obtaining the best cell yield along with the highest frequency of Th2 cells per well in IL-4 sufficient conditions (Figure 3. 5 A\&B). Future experiments were set up with a 1:4 BMDC to T cell ratio. 
Chapter Three: Investigating the potential role of IL-25 during the differentiation of Th2 cells

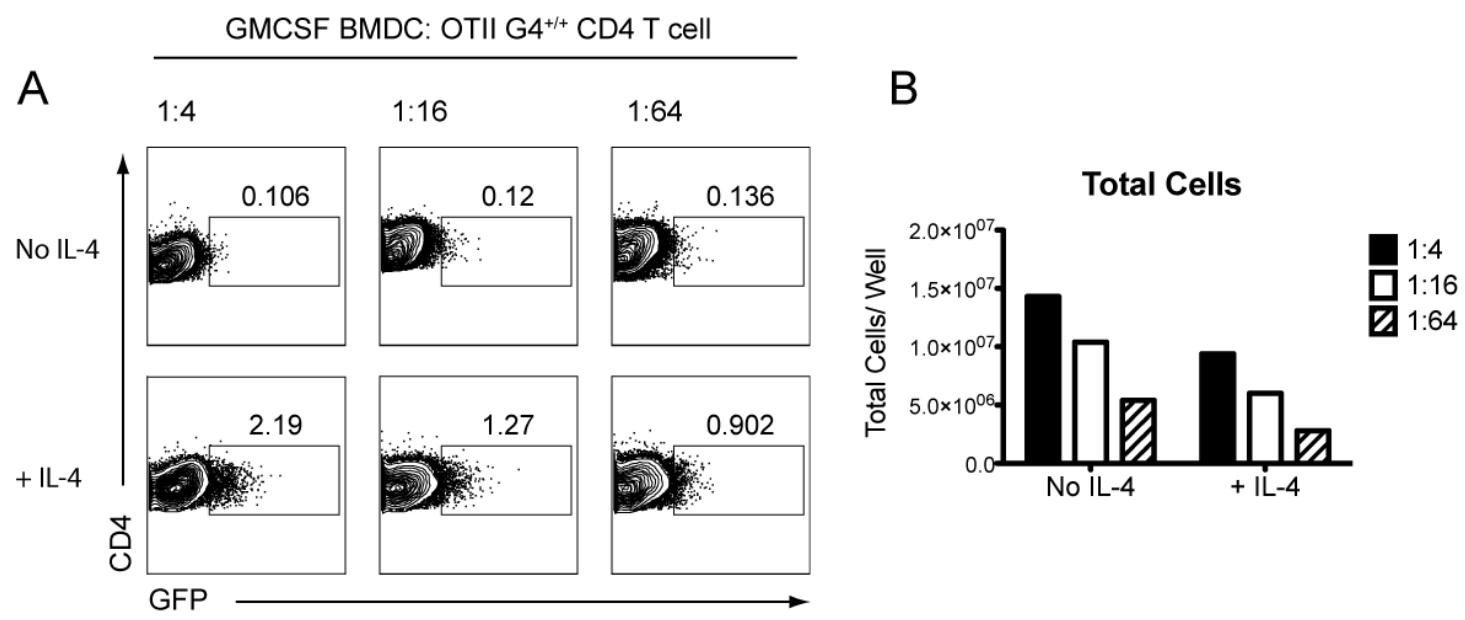

Figure 3. 5 BMDC to CD4 $\mathrm{T}$ cell ratio required for optimal cell counts and Th2 induction. OTII $\mathrm{G4}^{+/+}$reporter CD4 T cells were stimulated for 3 days with decreasing ratios of rGMCSF + rIL-4 BMDCs pulsed with $1 \mu \mathrm{M}$ ISQ in the presence or absence of rlL-4 $(1000 \mathrm{U} / \mathrm{ml})$. A: GFP ${ }^{+}$Th2 cells were determined by flow cytometry. B: Total cell yields were determined using Trypan blue exclusion dye. Data was compiled from one experiment with triplicate samples that were pooled and analysed.

\subsection{Optimisation of the in vitro assay for IL-4 independent differentiation of Th2} cells: IL-4 present in BMDC culture does not cross-contaminate T cell culture.

BMDCs were differentiated initially in rGMCSF and rIL-4 for 7 days prior to harvest and culture with naïve CD4 T cells; this initial culture system containing rIL-4 creates the possibility of IL-4 carry-over into the T cell system and therefore could account for Th2 cell differentiation that is noted in the absence of rIL-4 as seen in Figure 3. 5A Top Panel. To test whether IL-4 cross-contaminated the T cell culture, CD4 T cells that lacked STAT6, a crucial component in the IL-4 signalling pathway, were cultivated with GMCSF/IL-4 BMDC. Theorising that if IL-4 was responsible for the generation of Th2 cells then the STAT6 deficient CD4 T cells would not differentiate into Th2 cells and would not express GFP. A caveat to this experiment was the lack of access to STAT6 deficient animals backcrossed onto a C57BL/6 background resulting in the inability to cross them with the OTII TCR Tg animals that were only available on the C57BL/6 background. This called for an alternative strategy for a Th2 culture 
system that also made use of GMCSF/IL-4 BMDCs and could be used to query whether IL-4 was carried over from the BMDC culture into the T cell culture.

For this purpose an allogeneic Th2 differentiation system was designed whereby the BMDCs derived from IL-4 deficient C57BL/6 animals (from here on designated GMCSF/IL-4 C57BL/6 IL-4 ${ }^{-/}$BMDCs) and the CD4 T cells derived from IL-4 deficient $\mathrm{BALB} / \mathrm{c}$ animals (from here on designated $\mathrm{BALB} / \mathrm{c} \mathrm{G}^{+/+}$reporter $\mathrm{T}$ cells) would present self-antigens thereby driving CD4 T cell activation and differentiation. GMCSF/IL-4 C57BL/6 IL-4 ${ }^{-/}$BMDCs were co-cultured with BALB/c G4 ${ }^{+/+}$reporter T cells with or without intact STAT6 signalling, in the presence or absence of rIL-4. If contaminating IL-4 was carried over from the BMDC culture system then the BALB/c $\mathrm{G}^{+/+}$reporter $\mathrm{T}$ cells with intact STAT6 signalling would be expected to have significantly higher GFP expressing CD4 $\mathrm{T}$ cells than that of the BALB/c G4 $4^{++}$ reporter T cells without intact STAT6 signalling. However as can be noted in the top panel Figure 3. 6, the lack of STAT6 signalling in the CD4 T cells had no effect on the expression of GFP in the CD4 $\mathrm{T}$ cell population, illustrating the Th2 cell differentiation noted in the absence of rIL-4 was not as a result of a crosscontaminating source of IL-4 originating from the BMDC culture system. The lower panel serves as a control, illustrating lack of ability of STAT6 ${ }^{-1-} \mathrm{T}$ cells to respond to IL-4 signalling. 
Chapter Three: Investigating the potential role of IL-25 during the differentiation of Th2 cells

Balb/c T cells

C57BL/6
IL-4-
BMDC
Balb/c G4 $4^{+/+}$

Stat6 ${ }^{+/+}$T cells
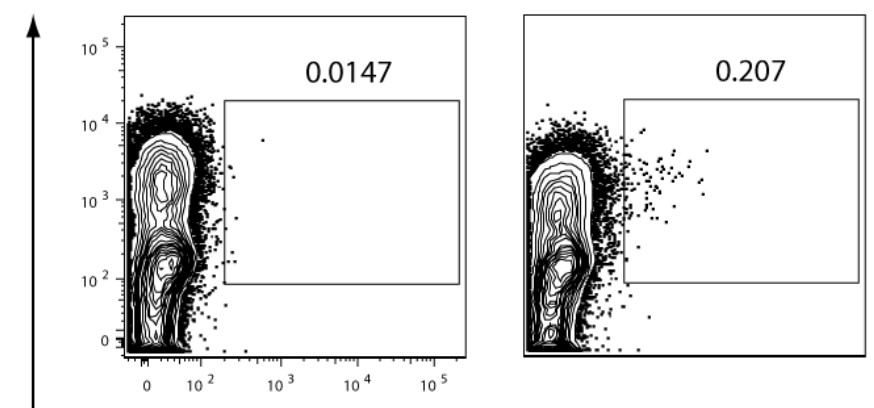

$+\mathrm{IL}-4$

莳

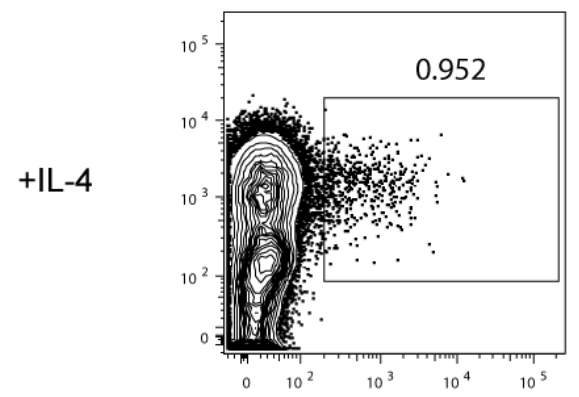

Balb/c G4 $4^{+/+}$

Stat6 ${ }^{-/} \mathrm{T}$ cells
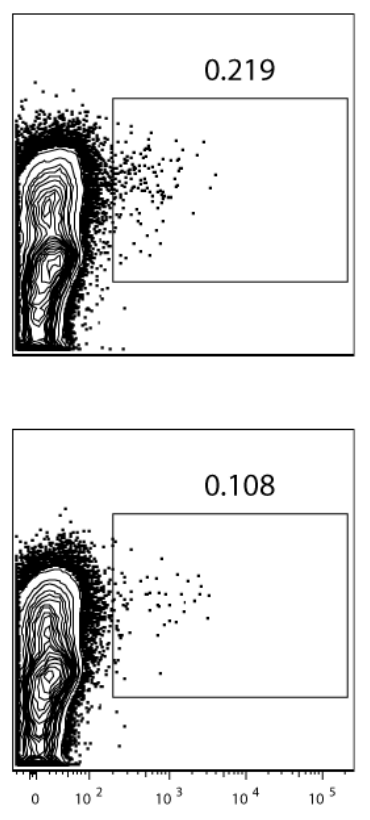

GFP

Figure 3. $6 \mathrm{IL}-4$ from the GMCSF/IL-4 BMDC culture is not responsible for differentiation of CD4 GFP ${ }^{+}$T cells. C57BL/6 IL-4 deficient GMCSF/IL-4 BMDCs were co-cultured with STAT6 deficient or wild type BALB/c G4 ${ }^{++}$CD4 T cells in rIL-2 $(50 \mathrm{U} / \mathrm{ml}$ ) with or without rlL-4 (1000 $\mathrm{U} / \mathrm{ml}$ ). Data is representative of at least 2 experiments with triplicate samples. 
Chapter Three: Investigating the potential polarising role of IL-25 during the differentiation of Th2 cells.

\subsection{Optimisation of the in vitro assay for IL-4 independent differentiation of Th2} cells: Maturing BMDCs with rIL-25.

DCs provide co-stimulation and release soluble signalling molecules that are responsible for polarising T helper cells. It is hypothesised DCs can be altered through a variety of maturation signals resulting in a DC phenotype that is more inclined to polarise Th2 cells ${ }^{202}$. To determine whether IL-25 could act as a signal that skews BMDCs to induce a stronger Th2 phenotype, the BMDCs were activated overnight in rIL-25 (150 ng/ml) and analysed for cell surface activation markers. The cytokine matured BMDCs showed no marked differences in CD11c, CD86 or MHC class II expression compared to the immature BMDCs (Figure 3. 7 A). These IL-25 matured BMDCs were co-cultured for 3 days with CD4 T cells in the absence of rIL-2 and in the presence or absence of rIL-4 (1000 U/ml) and CD4 T cells were analysed for GFP production. The IL-25 matured BMDCs were less effective at differentiating Th2 cells than LPS matured BMDCs (Figure 3. 7 B). In summary, maturation of BMDCs with the innate cytokine IL-25 provided no added benefits for increasing Th2 cell differentiation in this culture system. 
Chapter Three: Investigating the potential role of IL-25 during the differentiation of Th2 cells

A

IL-25 BMDC

LPS BMDC

Immature BMDC
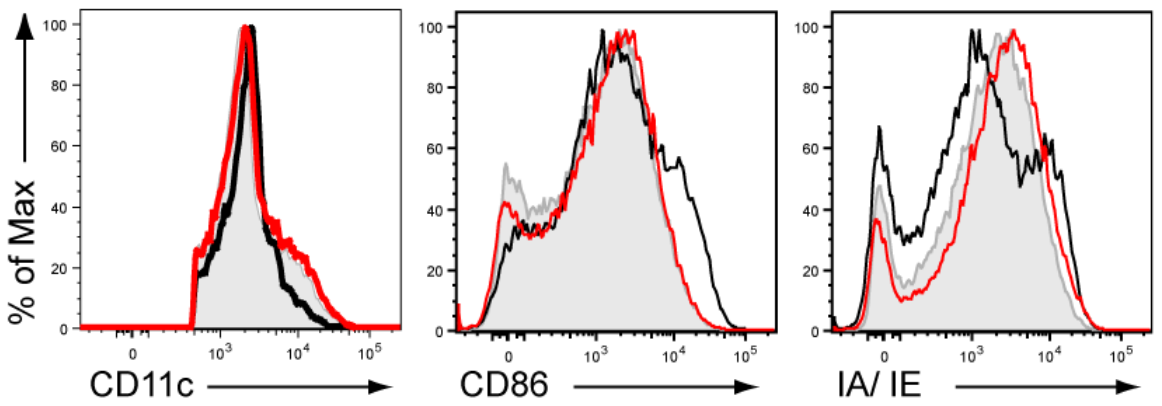

B

$1 \mu$ M ISQ Peptide.

No rlL-2 added

No IL-4

$+\mathrm{IL}-4$

to $T$ cell culture.

IL-25 BMDC

LPS BMDC

LPS BMDC

OTII

CD4 T cells
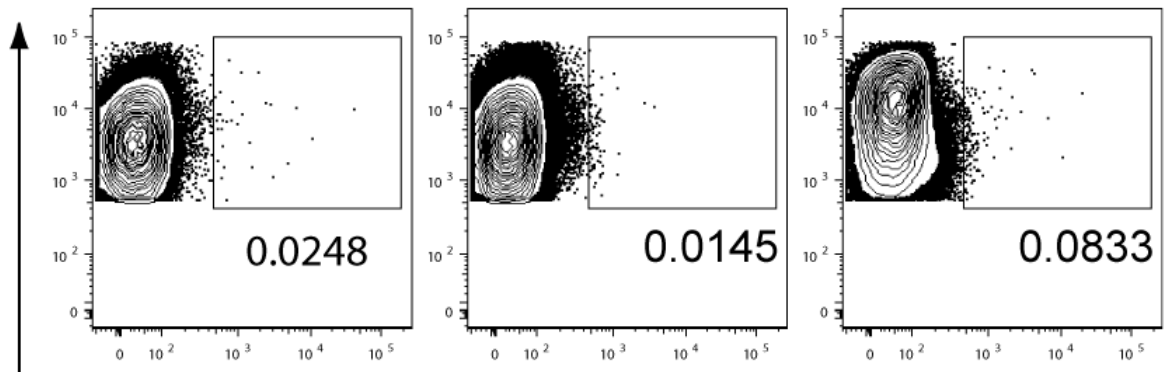

OTII G4+/+

CD4 T cells
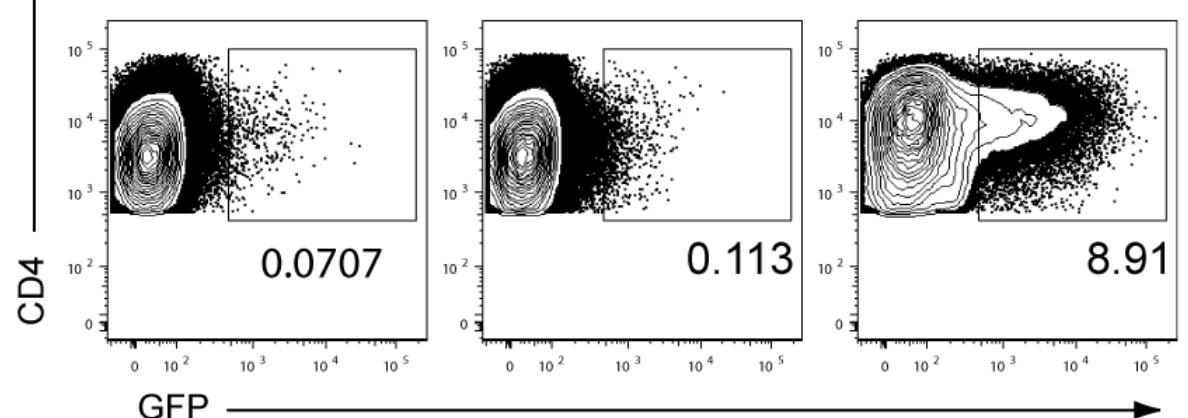

Figure 3. 7 Maturation of BMDCs for initiation of Th2 differentiation in vitro. Bone marrow derived dendritic cells were cultured with rGMCSF + rIL-4 then matured for 24hrs with rIL-25 $(150 \mathrm{ng} / \mathrm{ml})$ or LPS $(500 \mathrm{ng} / \mathrm{ml})$ A: Histograms depicting activation status of BMDCs $\underline{\text { B: }}$ Flow data shows the frequency of Th2 differentiated cells as illustrated by their ability to produce GFP. Data was compiled from one experiment with triplicate samples. 


\subsection{Optimisation of the in vitro assay for IL-4 independent differentiation of Th2} cells: Flt3L generated BMDCs.

GMCSF/IL-4 BMDCs resemble inflammatory dendritic cells ${ }^{203}$; however Flt3L generated BMDCs resemble the majority of DC subsets more commonly described in vivo ${ }^{204}$. As Flt3L BMDCs possibly represent a more physiologically relevant APC it was hypothesised they may differentiate Th2 cells more effectively than GMCSF/IL-4 elicited BMDCs. Flt3L BMDCs were pulsed with ISQ peptide and co-cultured with OTII G4 ${ }^{+/+}$CD4 T cells for 3 days in the presence of rIL-2 (1000 U/ml). Surprisingly, Th2 differentiation was barely detectable in cultures without the addition of rIL-4 (1000 U/ml) (Figure 3. 8). To determine whether the lack of Th2 differentiation noted in the culture system with only rIL-2 and no rIL-4 was due to a strong Th1 polarising stimulus such as the production of IL-12, an IL-12 blocking antibody was added to the culture. However blocking signalling of IL-12 resulted in no changes in Th2 or Th1 frequency, as determined by the production of IL-4 reporter protein GFP or IFN $\gamma$ respectively (Figure 3.8). Notably the Flt3L generated BMDCs required a cytokine milieu containing either the Th1 or Th2 polarising cytokine rIL-12 or rIL-4 respectively, for noticeable Th1 or Th2 differentiation as seen through the production of IFN $\gamma$ or IL-4 (GFP).

FIt3L BMDCs

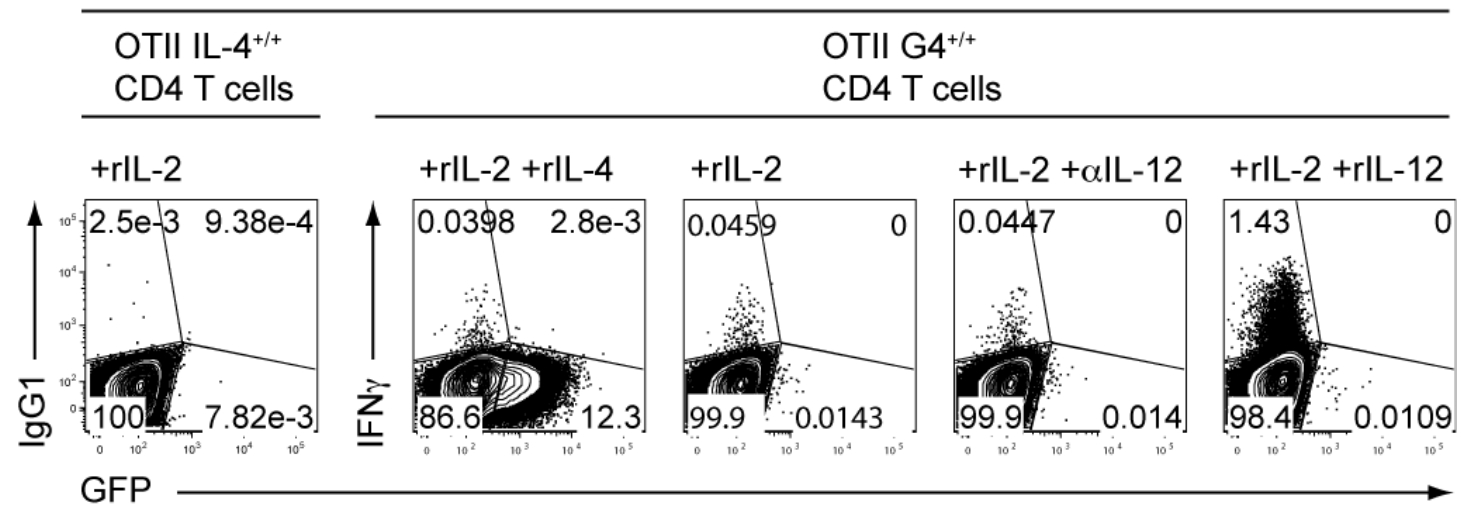

Figure 3. 8 Lack of frequency of Th2 cells by Flt3L DCs is not as a result of excessive production of IL-12 and Th1 differentiation. Flt3L BMDCs were pulsed with $1 \mu \mathrm{M}$ ISQ peptide and co-cultured with OTII G4 ${ }^{+/+}$CD4 T cells for 3 days in the presence of rIL-2 $(100 \mathrm{U} / \mathrm{ml})$ with or without rIL-4 $(1000 \mathrm{U} / \mathrm{ml}) / \alpha \mathrm{lL}-12(10 \mu \mathrm{g} / \mathrm{ml}) / \mathrm{rlL}-12(10 \mathrm{ng} / \mathrm{ml})$. Measured for the production of IFN $\gamma$ and GFP by flow cytometry. Data was compiled from one experiment with triplicate samples. 
Chapter Three: Investigating the potential role of IL-25 during the differentiation of $\underline{\text { Th2 cells }}$

\subsection{Optimisation of the in vitro assay for IL-4 independent differentiation of Th2 cells: Altering antigen concentration to promote Th2 differentiation.}

To determine what was the optimal antigen concentration for Th2 cell differentiation, ISQ peptide concentration was titrated in GMCSF/IL-4 and Flt3L BMDC cultures. Either GMCSF/IL-4 or Flt3L BMDCs were co-cultured with CD4 T cells in the presence of rIL-2 $(100 \mathrm{U} / \mathrm{ml})$ and ISQ peptide ranging from $10 \mu \mathrm{M}-0 \mu \mathrm{M}$. Titrating out the ISQ peptide resulted in notably decreased recoverable numbers of cells per well (Figure 3.9B), despite this decrease in cell numbers, the frequency of Th2 cells increased at the lower peptide concentrations (Figure 3.9A). This was followed by an experiment where antigen was titrated to even lower concentrations of $0.1 \mu \mathrm{M}-$ $0.001 \mu \mathrm{M}$ resulting in increased proportions of Th2 cell differentiation in the 0.001 $\mu \mathrm{M}$ CD4 T cell population (Figure 3. 10). In summary, lower antigen concentrations resulted in increased differentiation of Th2 cells but decreased proliferation rates and therefore lower recoverable yields of CD4 T cells per well. 


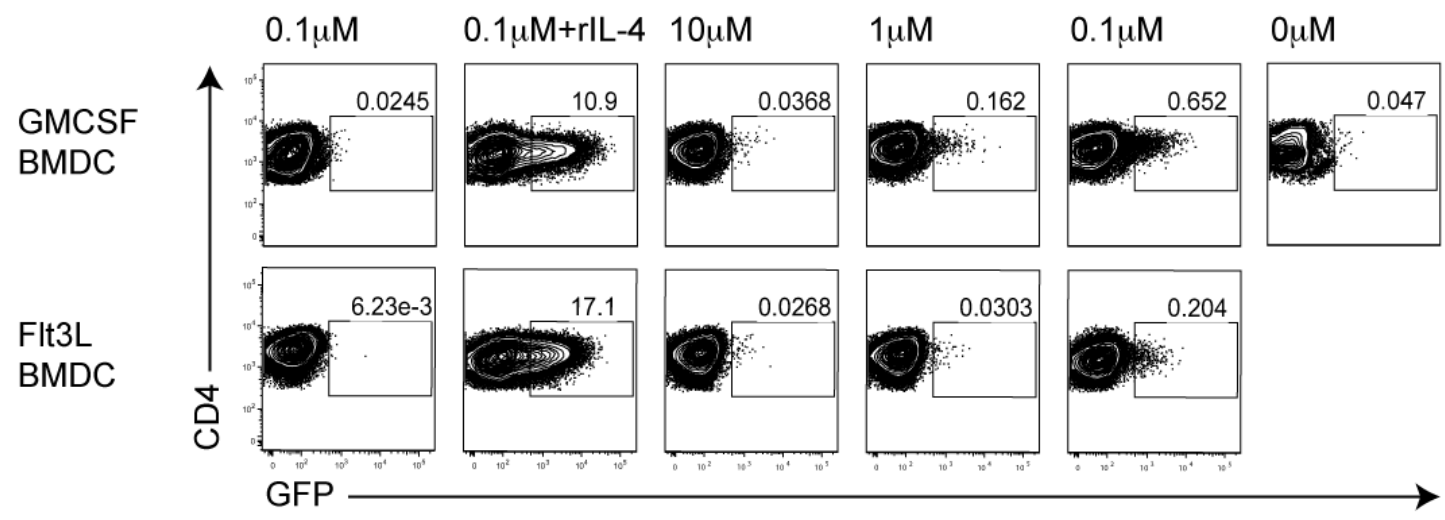

B

\section{GMCSF BMDC}

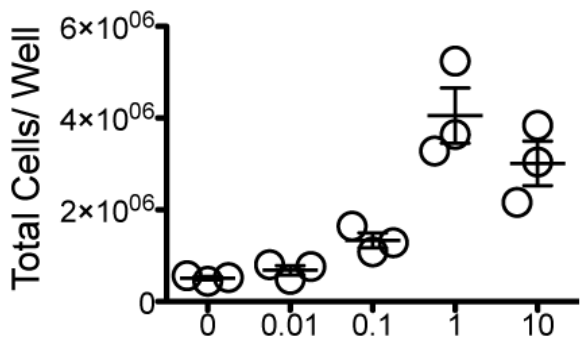

Concentration of Peptide $(\mu \mathrm{M})$
FIt3L BMDC

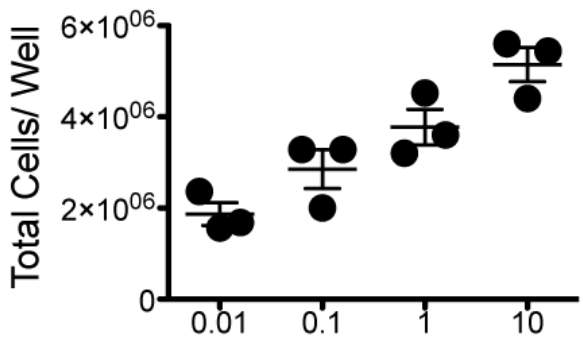

Concentration of Peptide $(\mu \mathrm{M})$

Figure 3. 9 Decreased antigen concentration results in increased Th2 frequency. GMCSF/IL-4 or Flt3L BMDCs were co-cultured with OTII G4 ${ }^{+/}$CD4 T cells in $\mathrm{rlL}-2(100 \mathrm{U} / \mathrm{ml})$ with/without rIL-4 $(1000 \mathrm{U} / \mathrm{ml})$ for 3 days in presence of decreasing concentrations of ISQ peptide. A: Th2 cells were determined by flow cytometry. B: Total viable cells were determined by trypan blue exclusion dye. Data is representative of at least 2 experiments where dot plots show mean \pm SEM of triplicate samples. 
Chapter Three: Investigating the potential role of IL-25 during the differentiation of Th2 cells

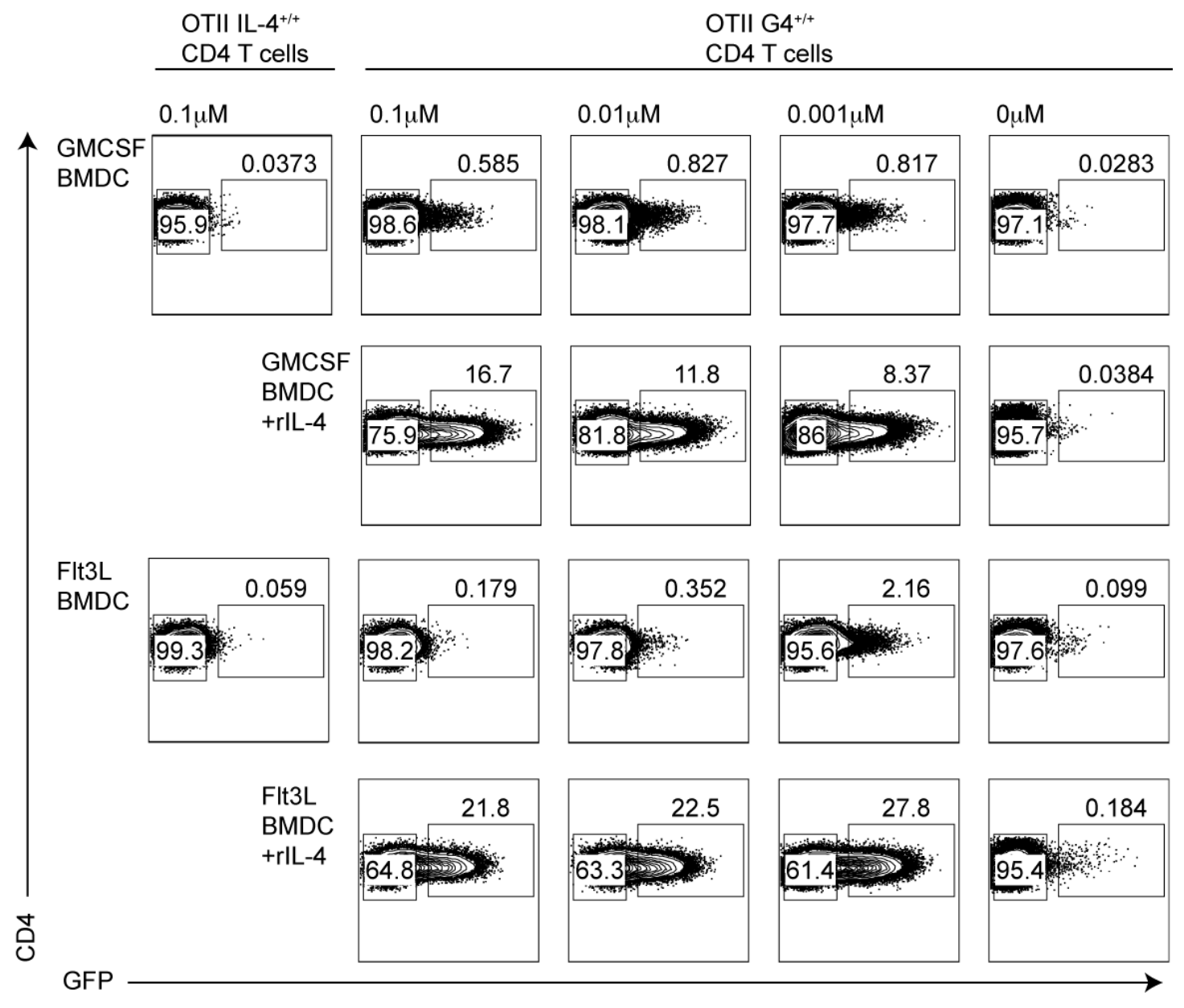

Figure 3. 10 Low concentrations of antigen are crucial for high Th2 frequencies. GMCSF/IL-4 or Flt3L BMDCs were co-cultured with OTII G4 ${ }^{+/+} \mathrm{CD} 4$ T cells in rIL-2 $(100 \mathrm{U} / \mathrm{ml})$ with/ without rIL-4 $(1000 \mathrm{U} / \mathrm{ml})$ for 3 days in presence of decreasing concentrations of ISQ peptide. GFP ${ }^{+}$ CD4 T cells (Th2) cells were determined by flow cytometry. Data is representative of at least 2 experiments with triplicate samples. 


\subsection{Investigating the role of IL-25 as an early differentiation factor in the antigen specific in vitro assay of IL-4 independent Th2 differentiation.}

The antigen specific culture system was developed to determine whether IL-25 was indeed a crucial factor for Th2 cell differentiation. Firstly, it was decided to ascertain whether DC-derived IL-25 was required for Th2 differentiation. In order to assess this, IL-25 $5^{-/}$GMCSF/IL-4 BMDCs were compared to IL-25 ${ }^{+/+}$BMDCs for their effectiveness in generating Th2 cells. IL- $25^{-/-}$BMDCs were cultured with OTII G4 ${ }^{+/+}$ CD4 $\mathrm{T}$ cells, ISQ peptide and rIL-2 $(100 \mathrm{U} / \mathrm{ml})$ for 3 days and subsequent GFP production by the CD4 T cells was analysed. The frequency of Th2 cells differentiated by $\mathrm{IL}-25^{-/}$BMDCs was slightly lower than the IL-25 $5^{+/+}$BMDC however neither in the presence nor absence of rIL-4 was Th2 cell differentiation ablated (Figure 3. 11). Moreover, the addition of rIL-25 or a blocking antibody to prevent signalling of IL-25 had little effect on Th2 differentiation (Figure 3. 11). In summary, these data clearly demonstrate there is no critical role for IL-25 during antigen specific in vitro differentiation of Th2 cells.

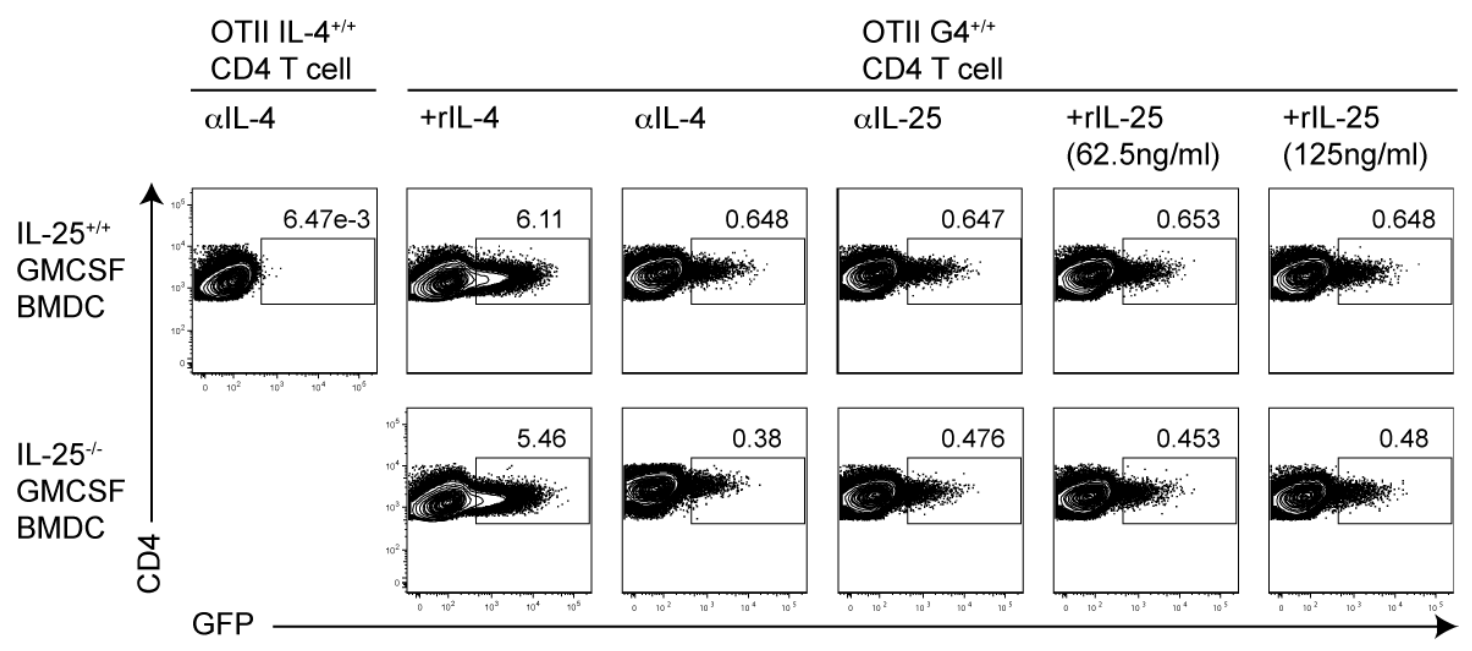

Figure 3. 11 Neither exogenous nor bone marrow dendritic cell derived IL-25 is required for Th2 differentiation. IL- $25^{+/+}$or IL- $25^{-/-}$BMDCs were pulsed with $0.1 \mu \mathrm{M}$ ISQ peptide and cocultured with OTII G4 ${ }^{+/+}$CD4 T cells for 3 days in rIL-2 $(100 \mathrm{U} / \mathrm{ml})$ with or without rlL-4 (1000 $\mathrm{U} / \mathrm{ml}) / \alpha \mathrm{LL}-25(10 \mu \mathrm{g} / \mathrm{ml}) / \mathrm{rlL}-25$ (62.5 or $125 \mathrm{ng} / \mathrm{ml}$ ) and analysed by flow cytometry for $\mathrm{GFP}^{+}$CD4 T cells (Th2) cells. Data is representative of at least 2 experiments with triplicate samples. 
Chapter Three: Investigating the potential role of IL-25 during the differentiation of Th2 cells

\subsection{Optimisation of the in vivo assay for differentiation of Th2 cells using worm derived allergens.}

To investigate whether IL-25 is required for Th2 in vivo differentiation, an assay in which all Th2 differentiation events are contained within a single draining lymph node was utilised. To determine when the initial differentiation of Th2 cells can be detected in this single draining lymph node assay and to confirm that the absence of IL-4 signalling has no effect on Th2 differentiation in an in vivo setting, 600 dead L3 $N$. brasiliensis were injected into the dermis of the ear of IL-4 sufficient G4 ${ }^{+-}$and IL-4 deficient $\mathrm{G}^{+/+}$mice. The ear draining lymph nodes were harvested daily following dead L3 $N$. brasiliensis injection and single cell suspensions were analysed by flow cytometry for $\mathrm{GFP}^{+} \mathrm{CD} 4 \mathrm{~T}$ cells (Figure 3. 12A), illustrative of the Th2 cell population. The first distinct Th2 cell population was easily discernable in the ear draining lymph node at day 3 after injection (Figure 3. 12A). As previously reported ${ }^{65}$ the differentiation of Th2 cells occurs in the absence of IL-4 signalling as Th2 cells differentiate in $\mathrm{G}^{+/+}$mice (Figure 3. 12A). CD4 T cells were harvested 4 days after administration of 600 dead L3 $N$. brasiliensis into the ear dermis of $\mathrm{G} 4^{+/} \mathrm{IL}-25^{+/+}$mice and $\mathrm{G} 4{ }^{+/-} \mathrm{IL}-25^{-/}$mice. These cells were subsequently re-stimulated, and stained for intracellular IL-4 and assessed by flow cytometry. The intracellular staining clearly depicted no deficiency in either reporter protein or IL-4 protein expression in $\mathrm{G} 4^{+/} \mathrm{IL}-$ $25^{-/}$animals when compared to $\mathrm{G}^{+/-} \mathrm{IL}^{-25^{++}}$control (Figure 3. 12B). In summary, Th2 initiation occurs by day 3 after challenge with worm antigens, and this initiation is not affected by the absence of IL-25 signalling.

\subsection{Optimisation of the in vivo assay for differentiation of Th2 cells using worm derived allergens: Determining expression of Th2 associated cytokines.}

The CD4 T cell populations that differentiated in the absence of IL-25 were analysed for Th1 and Th2 cytokines to determine if loss of IL-25 signalling resulted in a skewing from Th2 to Th1 differentiation. Four days following dermal challenge with 600 dead L3 N. brasiliensis the ear draining lymph nodes were harvested, processed and analysed by flow cytometry for the hallmark Th1 cytokine IFN $\gamma$, as well as the T 
helper type 2 cytokines IL-5 and IL-13. Frequencies of IFN $\gamma$, IL-5 and IL-13 were similar in IL-25 sufficient and deficient animals inferring there is no role for IL-25 in the production of these cytokines following challenge with worm antigens (Figure 3. $13)$.

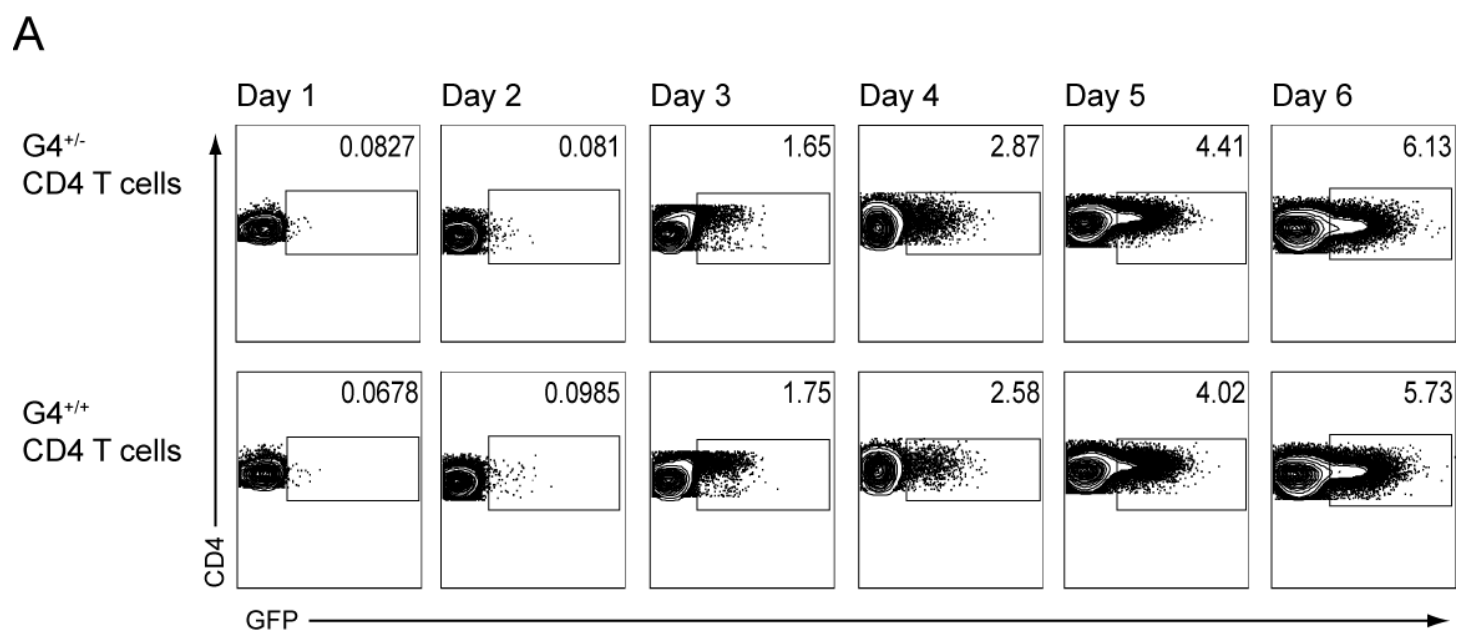

B

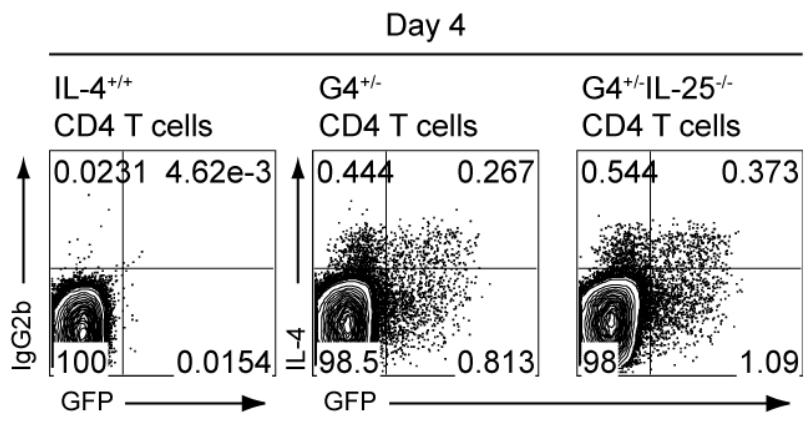

Figure 3. 12 Th2 cells can be tracked over time in response to intradermal adminstration of type 2 inducers. Ear draining lymph nodes were harvested at indicated times after ear intradermal administration of 600 dead L3 N. brasiliensis. CD4 T cells were analysed by flow cytometry for $\underline{\mathbf{A}}$ : the production of GFP as a surrogate for IL-4 protein $\underline{\mathbf{B}}$ : intracellular IL-4 and compared to GFP reporter production following $4 \mathrm{hrs}$ of polyclonal re-stimulation. Data is representative of at least 2 experiments with $n \geq 4$ mice per group. 
Chapter Three: Investigating the potential role of IL-25 during the differentiation of Th2 cells

\section{Day 4}

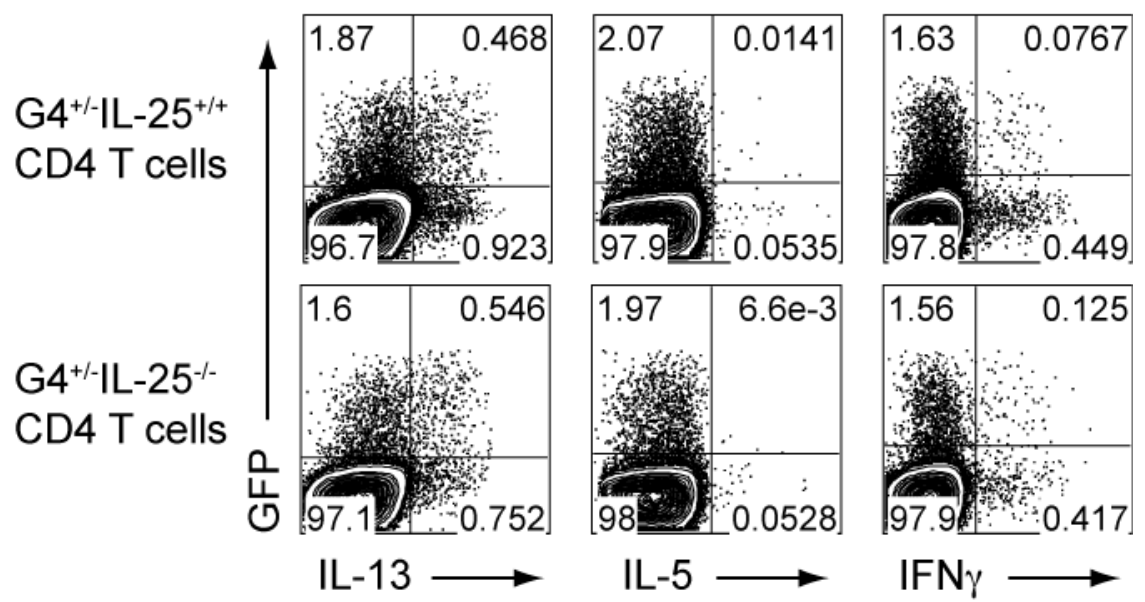

Figure 3. 13 Lack of IL-25 signalling has no effect on the expression of IL-4, IL-5 or IFN $\gamma$ in response to worm antigens. Ear draining lymph nodes were harvested after intradermal administration of 600 dead L3 N. brasiliensis. CD4 T cells were re-stimulated, intracellular stained and analysed by flow cytometry for IL-13, IL-5 and IFN $\gamma$ in comparison to the expression of GFP the reporter protein for IL-4. Data is representative of at least 2 experiments with $n \geq 4$ mice per group.

\subsection{Using the in vivo assay for CD4 $\mathrm{T}$ cell differentiation to investigate the role} of IL-25 in Th2 differentiation.

As the earliest detectable induction of Th2 cells in the ear draining lymph node was seen 3 days following injection of dead $N$. brasiliensis, this time point was chosen to further investigate the differentiation of Th2 cells in IL-25 or IL-25 and IL-4 deficient conditions. Two Th2 inducers were utilised, $N$. brasiliensis derived allergens and a common allergen house dust mite. Comparing $\mathrm{GFP}^{+} \mathrm{CD} 4 \mathrm{~T}$ cell frequencies generated in response to dead $N$. brasiliensis or house dust mite in $\mathrm{G} 4^{+/} \mathrm{IL}-25^{-/-}$mice compared to $\mathrm{G}^{+/}{ }^{-} \mathrm{IL}-25^{+/+}$mice showed no differences (Figure 3.14 A). Even in double cytokine deficient mice, which lack both IL-4 and IL-25 (G4 ${ }^{+/+} \mathrm{IL}-25^{-/-}$mice $)$, there were no differences in Th2 cell frequencies (Figure 3. $14 \mathrm{~B}$ ). These $\mathrm{GFP}^{+} \mathrm{CD} 4 \mathrm{~T}$ cells were also analysed for activation marker CD44 and were found to express similar levels of CD44 on the cell surfaces of Th2 cells whether they were differentiated in the absence of IL-25 alone or both IL-4 and IL-25 compaired to sufficient animals (Figure 3. 14 
A\&B). Overall, these results show there was no role for IL-25 during Th2 cell differentiation in response to common innocuous allergens or worm-derived allergens.

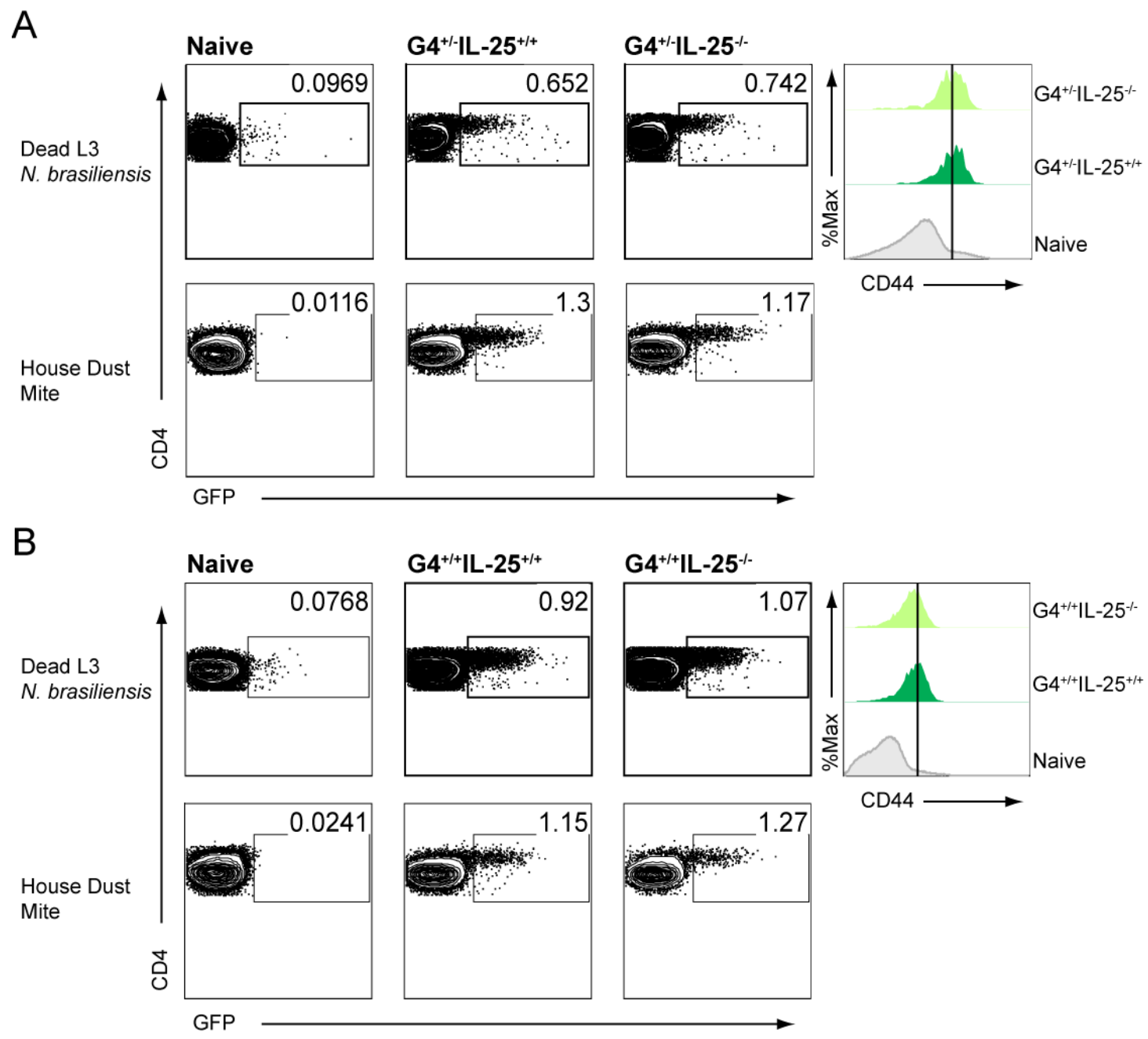

Figure 3. 14 IL-25 is not necessary for Th2 differentiation in vivo. Ear draining lymph nodes were harvested on day 3 after intradermal administration of 600 dead L3 N. brasiliensis or house dust mite. CD4 T cells were analysed by flow cytometry for the production of GFP and the expression of the activation marker CD44 on GFP ${ }^{+}$cells. $\underline{A}$ : In the presence of IL-4 signalling $\underline{B}$ : In the absence of IL-4 signalling. Data is representative of at least 2 experiments with an $n \geq 4$ mice per group. 
Chapter Three: Investigating the potential role of IL-25 during the differentiation of Th2 cells

\subsection{Discussion}

The cellular and molecular events involved in the differentiation of Th2 cells are still not fully understood. Traditionally, IL-4 was believed critical for the differentiation of Th2 cells ${ }^{8,192,205}$. However more recently, in vivo data has emerged which demonstrates that Th2 cells differentiate in vivo without the need for IL-4 ${ }^{65}$. The aim of this chapter was to examine whether the innate cytokine IL-25 was a viable candidate for differentiating Th2 cells in the absence of IL-4. Utilising sensitive reporter mice, IL-4 independent Th2 cells were shown to differentiate in vitro following polyclonal activation, albeit to a significantly lower frequency than those differentiated in the presence of IL-4. To increase this IL-4 independent in vitro differentiation, an antigen specific system was developed and optimised to induce the highest frequency of IL-4 reporter producing CD4 T cells. Using this antigen specific activation system the role of IL-25 on BMDC maturation and Th2 cell differentiation was investigated. Of particular interest was the potential of IL-25 as an in vivo differentiation factor. An assay of early in vivo Th2 differentiation was developed, however the absence of IL-25 had no effect on the differentiation of Th2 cells.

The discovery of polyclonal in vitro IL-4 independent Th2 cells was surprising; especially considering previous research has described polyclonal activation as insufficient for the differentiation of Th2 cells in the absence of IL-4 ${ }^{65}$. It was decided to utilise a CD4 $\mathrm{T}$ cell antigen specific differentiation system to ensure the GFP producing cells were indeed $\mathrm{CD} 4^{+} \mathrm{Th} 2$ cells and not $\mathrm{CD}^{+}$iNKT cells. The use of TCR transgenic CD4 $\mathrm{T}$ cells for activation in an antigen specific response and resulting in Th2 differentiation in the absence of IL-4 has been reported in a 6 day culture system ${ }^{206}$, this chapter placed a novel twist on these data by investigating the earliest detectable consistent IL-4 production as well as avoiding the need for restimulation, fixation or intracellular staining to visualise this IL-4 production through the use of G4 reporter mice. The antigen specific IL-4 independent in vitro Th2 differentiation was notably reduced in frequency and median fluorescent intensity as compared to IL-4 sufficient Th2 differentiated CD4 T cells. This is in stark contrast to the data gathered in vivo in the ear draining lymph node following challenge with 
worm allergens where no differences were noted in the differentiation of Th2 cells in the presence or absence of IL-4.

To increase the frequency of differentiation of Th2 cells in vitro in the absence of IL-4 signalling, antigen, cytokines and DC phenotypes were altered. Lowering the concentration of antigen resulted in increased $\mathrm{Th} 2$ cell differentiation however the frequency of differentiation never increased to frequencies similar to those of the CD4 $\mathrm{T}$ cells differentiated in the presence of IL-4. Alternate optimisation techniques were attempted such as maturing DCs with the innate cytokine IL-25 in an attempt to skew the DC to produce more favourable Th2 stimulatory signals. However this resulted in no obvious differences to the frequency of Th2 cell differentiation. Alteration of BMDC type from GMCSF + IL-4 'inflammatory' DCs to Flt3L 'plasmacytoid and common' DCs enhanced the frequency of Th2 initiation, but only at antigen concentrations that were lower than $1 \mu \mathrm{g} / \mathrm{ml}$. Notably even the addition of rIL-25 directly into the $\mathrm{T}$ cell culture system had no effect on Th2 differentiation. This disagrees with previous literature reporting the addition of rIL-25 to naïve CD4 T cells results in increased IL-4 production ${ }^{81,197}$. This discrepancy possibly arises due to the different techniques used to measure IL-4 production. In this chapter IL-4 is measured on a single cell basis, through the use of reporter proteins, whereas the previous reports measured IL-4 by ELISA and did not account for the increasing numbers of cells due to proliferation in the presence of rIL-25. It is reasonable to assume the increase in IL-4 detectable in the presence of rIL-25 was due to the increased numbers of CD4 $\mathrm{T}$ cells resulting from $\mathrm{T}$ cell proliferation to IL-25 rather than an increased frequency of Th2 cell differentiation. This chapter conclusively illustrates that IL-25 was not the initiating factor required for in vitro IL-4 independent Th2 differentiation.

Unlike in vitro differentiation, differentiation of Th2 cells in vivo is equivalent in magnitude, timing and character in the presence or absence of IL-4. This may suggest additional signals are present in vivo that fail to be replicated in vitro. The question raised in this chapter was whether this additional factor was IL-25, acting either on the APC or directly on the naïve CD4 T cells. The initiation of Th2 cells took three days to develop following challenge with antigen. The absence of IL-25 had no effect on the differentiation of these Th2 cells, whether or not IL-4 was present. This 
Chapter Three: Investigating the potential role of IL-25 during the differentiation of Th2 cells

differentiation was not due to an immune modulating property of the worm derived antigens as even the common allergen house dust mite was effective in differentiating Th2 cells in the absence of IL-25. In summary, the data herein illustrates IL-25 is not the polarisation factor that results in Th2 cell differentiation and the production of IL4. 
Chapter Four:

The role of IL-25 in the generation of protective T helper type 2 responses against infection by parasitic helminths. 
Chapter Four: The role of IL-25 in the generation of protective T helper type 2 responses against infection by parasitic helminths.

\subsection{Background}

Administration or over-expression of recombinant IL-25 results in Th2 associated pathologies including airway hyper-responsiveness, goblet cell hyperplasia and mucous hyper-secretion, as well as the rapid expulsion of intestinal stage Nippostrongylus brasiliensis ${ }^{137,138,175,196}$. Additionally, studies using IL-25 deficient mice demonstrate that the induction of both Th2 innate and adaptive responses are impaired resulting in the delayed expulsion of $N$. brasiliensis from the small intestine ${ }^{175}$ or decreased resistance to Trichuris muris ${ }^{145}$. Findings such as these have led to the speculation that IL-25 may be a key component during the generation of $\mathrm{Th} 2$ immunity.

Helminth infections result in a robust Th2 immune response and are thought to represent an evolutionary pressure for the development of Th2 immunity. The studies herein utilise two helminth infections, a chronic entirely enteric helminth, Heligmosomoides polygyrus, and an acute helminth, Nippostrongylus brasiliensis, whose lifecycle traverses through the skin, lung and intestine. Importantly, both are dependent upon the generation of Th2 responses for protective immunity ${ }^{163,207}$. Protective immunity has often been described as the ability to expel primary intestinal stage worms, however in this study protective immunity is defined as the ability to mount a rapid and effective memory response against re-infection that results in lower worm burdens and faster resolution of infection. The precise effector mechanisms required for protective immunity are poorly understood and with no effective vaccines available against helminth infections and a third of the world's population burdened by parasitic worm infections, research to better understand mechanisms of protective immunity remains a necessity. Given the known role IL-25 plays in timely expulsion of intestinal phase helminths following primary infection, this cytokine presents itself as a strong potential candidate involved in mediating protective Th2 immunity.

CD4 T cells are vital in the generation of Th2 immunity against $N$. brasiliensis, with a specific requirement for their presence during primary infections to enable protective responses to secondary infections ${ }^{169,208}$. The two key cytokines required for protective immunity are IL-4 and IL-13. Expulsion of primary N. brasiliensis from the intestine 
is critically dependent upon IL-13 ${ }^{168}$, recent data suggests the source of IL-13 in this setting is acquired from a newly discovered lineage negative innate lymphoid cell subset (ILC2) ${ }^{61,150-152}$. IL-13 drives a "weep and sweep" mechanism resulting in increased intestinal contractility along with increased mucous and luminal fluid all resulting in the expulsion of the intestinal stage adult worms ${ }^{209}$. During challenge infections protective mechanisms occur long before $N$. brasiliensis even reaches the small intestine with significantly lower worm burdens evident in the lungs within 24 hours of the challenge infection ${ }^{161}$ and yet this early mechanism of protection remains largely a mystery, although a critical role for IL-4 and it signalling pathway STAT6 have been described ${ }^{161}$.

During primary infection basophils are the dominant source of IL-4 while Th2 cells the predominant contributors of IL-4 during secondary infections ${ }^{55}$. In the past year two published reports have suggested Th2 cells preferentially secrete IL-4 in the lymph nodes and IL-13 in the lungs ${ }^{210,211}$, bringing into question whether Th2 cells are the dominant source of IL-4 during secondary infections. Of course there is the possibility that the production of IL-4 by Th2 cells in the lymph node is sufficient for the generation of protection from a challenge infection. However, there is a novel potential cellular source of early lung IL-4 in the form of the ILC2s, a source of IL-4 never previously investigated during secondary infections to $N$. brasiliensis. Of the ILC2 cells described, nuocytes are the most likely candidates as they have been described to produce IL-4 and assist with helminth mediated protection ${ }^{61}$. The natural helper cells share so many similarities with the nuocytes it is likely these cells are the same subset ${ }^{152}$. The innate helper type 2 (Ih2) cells are, however, unlikely contributors to this source of IL-4 as they have been described exclusively as IL-13 producers that do not produce IL-4 ${ }^{150}$ and the multipotent progenitor type 2 cells differentiate into a number of other cell types thereby not representing a mature effector subset ${ }^{151}$. Therefore this chapter seeks to investigate whether in the absence of IL-25 either Th2 cells or nuocytes are reduced in their ability to produce IL-4 thereby resulting in loss of protection against challenge infections by helminths. 
Chapter Four: The role of IL-25 in the generation of protective T helper type 2 responses against infection by parasitic helminths.

\subsection{Research Objectives}

The aim of this chapter is to determine whether IL-25 has a role in protective type 2 immune responses against parasitic infections. The experiments described herein specifically focus on whether IL-25 is required for the differentiation and maintenance of $\mathrm{Th} 2$ cells and the stabilisation of typical $\mathrm{Th} 2$ immune responses including mucous hyperplasia, eosinophilia, basophilia and B cell class-switching to IgE. The intent of this chapter was to investigate whether a novel innate lymphoid cell population known as nuocytes, which are reported to proliferate and become functionally active in response to rIL-25, are responsible for the helminth protective immunity generated in the lungs and gut.

Summarised aims:

- Establish an in vivo assay that allows us to investigate whether IL-25 is crucial for the distribution and development of Th2 cell effector or memory formation in lymphoid and non-lymphoid tissues.

- Establish whether IL-25 is necessary for helminth associated type 2 responses including IgE isotype switching, mucous production, cytokines and recruitment of effector cells.

- Establish whether IL-25 is required for the differentiation and expansion of nuocytes in the lung and draining lymph nodes following $N$. brasiliensis infection.

- Establish whether IL-25 has a role in protective immunity against challenge infections of $N$. brasiliensis and H.polygyrus. 


\subsection{Results}

\subsection{Helminth induced Th2 cells are not altered by the absence of IL-25}

To determine whether IL-25 plays a role in the differentiation and maintenance of Th2 cells in a natural immune response setting, $\mathrm{G} 4^{+/-}, \mathrm{G}^{+/-} \mathrm{IL}^{-25^{-/}}, \mathrm{G}^{+/+}$and $\mathrm{G} 4^{+/+} \mathrm{IL}^{-25^{-/}}$ mice were subcutaneously infected with L3 stage $N$. brasiliensis and left for 45 days. At day 45 prior to re-infection (PTR), these animals were challenged again with L3 stage $N$. brasiliensis resulting in the rapid expansion of Th2 cells, which were easily monitored in the G4 reporter mice (Figure 4. 1). To determine whether IL-25 played a prominent role during the induction, maintenance or localisation of these Th2 cells, the kinetics of Th2 cells during a primary and memory Th2 immune response to parasitic infection were monitored in both lymphoid and non-lymphoid tissues (Figure 4. 1 and Figure 4. 2 respectively).

Flow cytometic analysis of mediastinal lymph nodes and lungs allowed for the enumeration of individual Th2 $\left(\mathrm{CD}^{+} \mathrm{CD}^{+} \mathrm{GFP}^{+}\right)$cells that developed over the course of both the primary and secondary $N$. brasiliensis infections. In both the IL-4 sufficient (Figure 4. 1A and Figure 4. 2A) and IL-4 deficient (Figure 4. 1B and Figure 4. 2B) mice, the lack of IL-25 had little affect on the over-all kinetics of Th2 differentiation. In both the mediastinal lymph nodes and lungs, Th2 cells were detectable in these animals in similar frequencies and numbers with the only differences noted in the numbers of the Th2 cells in the later time points of the lungs following secondary infection. Overall, these data illustrated that the absence of IL-25 had no affect on the kinetics of Th2 cell differentiation in the mediastinal lymph node or lung although they do illustrate a possible role for IL-25 in tissue resolution of Th2 cell responses. 
Chapter Four: The role of IL-25 in the generation of protective T helper type 2 responses against infection by parasitic helminths.

\section{A: IL-4 Sufficient}

(i). Frequency of Th2 cells

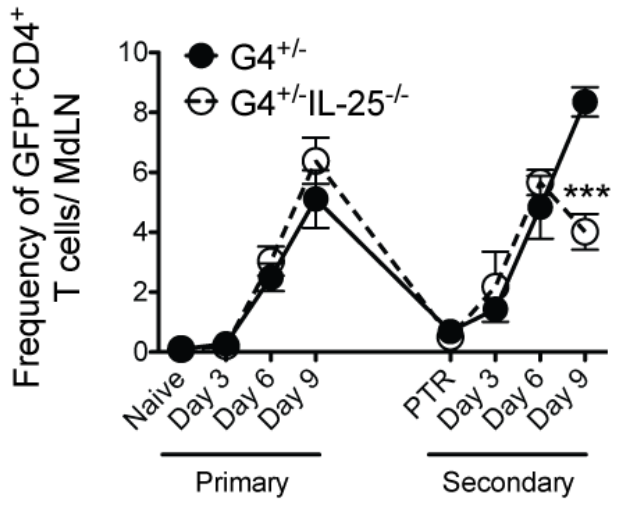

N. brasiliensis infection (ii). Numbers of Th2 cells

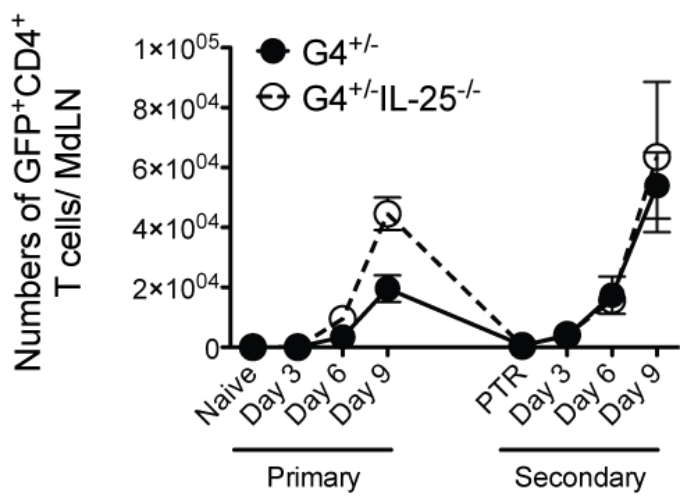

N. brasiliensis infection

\section{B: IL-4 Deficient}

(i). Frequency of Th2 cells

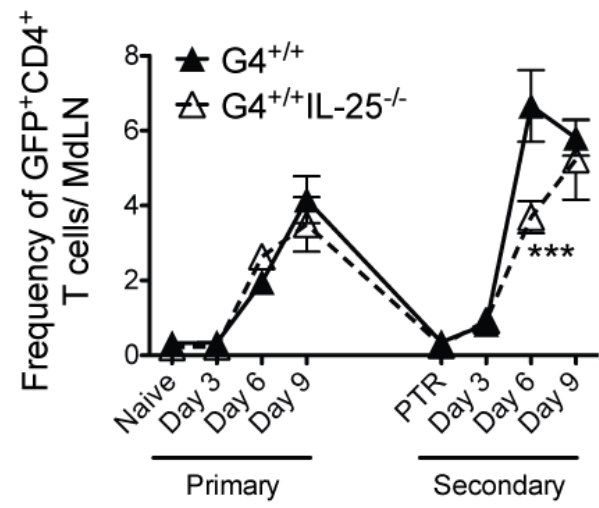

N. brasiliensis infection

(ii). Numbers of Th2 cells

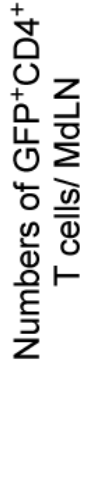

N. brasiliensis infection

Figure 4. 1 II-25 is not required for development of Th2 cells in a Th2 effector or memory T cell mediastinal lymph node response setting Mediastinal lymph nodes (MdLN) were harvested 3, 6 and 9 days following primary infection with 600 L3 SQ N. brasiliensis. Mice were challenged at 45 days after primary infection and MdLNs were harvested 3, 6 and 9 days later to determine secondary immune responses. MdLNs were processed into single cell

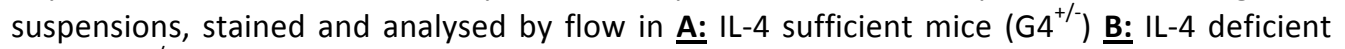
mice $\left(\mathrm{GS}^{+/+}\right)$(i). Frequency of Th2 cells (Doublet DAPI $\mathrm{CD}^{+} \mathrm{CD}^{+} \mathrm{GFP}^{+}$cells) and (ii). Total numbers of Th2 cells per lymph node. ${ }^{*} p \leq 0.01,{ }^{*} p \leq 0.001, * * * p \leq 0.0001 ; 2$ Way ANOVA with a Bonferroni post test as compared to $\mathrm{G}^{+/-}$or $\mathrm{G}^{+/+}$Th2 cells. Line graphs show mean $\pm \mathrm{SEM}$, $\mathrm{n} \geq 4$ mice/group. PTR: prior to re-infection. MdLN: Mediastinal lymph node. 


\section{A: IL-4 Sufficient}

(i). Frequency of Th2 cells

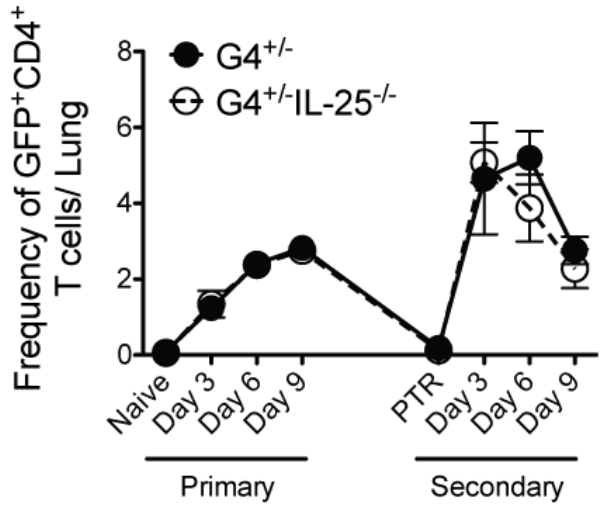

N. brasiliensis infection (ii). Numbers of Th2 cells

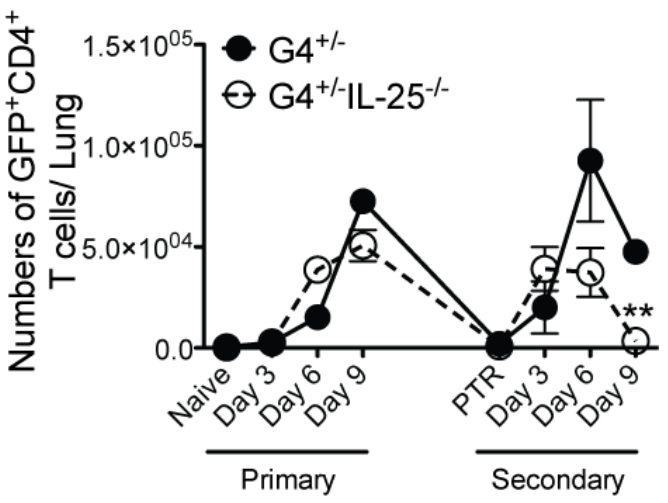

N. brasiliensis infection

\section{B: IL-4 Deficient}

(i). Frequency of Th2 cells

(ii). Numbers of Th2 cells

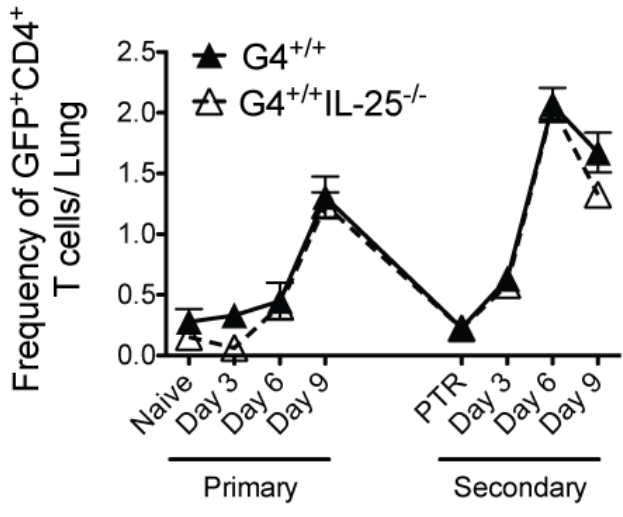

N. brasiliensis infection

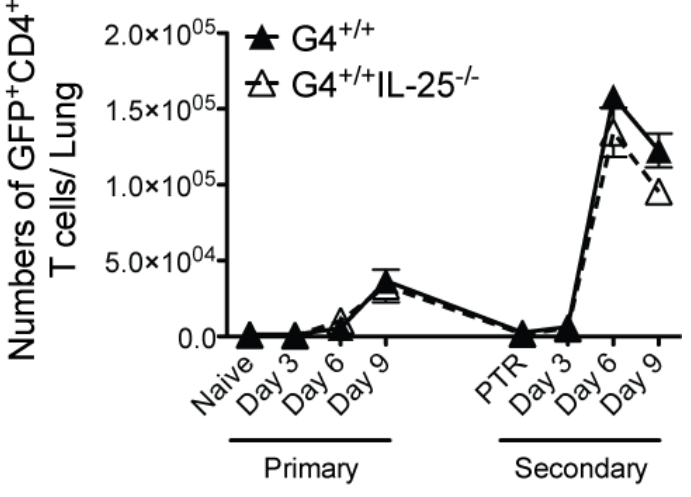

N. brasiliensis infection

Figure 4. 2 II-25 is not required for development of Th2 cells in a Th2 effector or memory T cell lung response setting. Lungs were harvested 3, 6 and 9 days following primary infection with 600 L3 SQ N. brasiliensis. Mice were challenged at 45 days after primary infection and lungs were harvested 3, 6 and 9 days later to determine secondary immune responses. Lungs were processed into single cell suspensions, stained and analysed by flow in A: IL-4 sufficient mice $\left(\mathrm{G}^{+/-}\right)$B: IL-4 deficient mice $\left(\mathrm{G}^{+/+}\right)$(i). Frequency of Th2 cells (Doublet'DAPI $\mathrm{CD}^{+} \mathrm{CD}^{+} \mathrm{GFP}^{+}$cells) and (ii). Total numbers of Th2 cells per lung. ${ }^{*} \mathrm{p} \leq 0.01,{ }^{* *} \mathrm{p} \leq 0.001$, *** $\mathrm{p} \leq 0.0001 ; 2$ Way ANOVA with a Bonferroni post test as compared to $\mathrm{G}^{+/-}$or $\mathrm{G}^{+/+} \mathrm{Th} 2$ cells. Line graphs show mean $\pm S E M, n \geq 4$ mice/group. PTR: prior to re-infection. 
Chapter Four: The role of IL-25 in the generation of protective T helper type 2 responses against infection by parasitic helminths.

\subsection{Type 2 cytokine profile in the lung is not altered by the lack of IL-25}

To determine whether IL-25 was responsible for promoting or maintaining Th2 associated cytokines or suppressing Th1 and Th17 cytokines, as has previously been reported ${ }^{145,194}$, IL-4, IL-5, IL-9, IL-13, IL-17 and IFN $\gamma$ were assessed. Lung cells from day 9 at the peak of the primary Th2 inflammatory response and at day 9 just after the peak of the secondary Th2 inflammatory response of infected IL-25 $5^{+/+}$and IL-25 animals were re-stimulated on $\alpha \mathrm{CD} 3 / \alpha \mathrm{CD} 28$ in the presence of rIL-2 for $24 \mathrm{hrs}$. The supernatants were evaluated by multiplex cytokine analysis to determine whether the loss in IL-25 signalling resulted in anomalies in cytokine production. Due to the time point chosen during the secondary response, the cytokine production was markedly lower than that detectable during the primary as the Th2 immune response has already began to contract. Remarkably Th2 associated cytokines IL-4, IL-13, IL-5 and IL-9 showed no significant difference between controls and IL-25 deficient animals (Figure 4. 3). The Th1 cytokine, IFN $\gamma$, was significantly reduced while no significant difference was noted in the production of the Th17 cytokine, IL-17, in the IL-25 sufficient compared to deficient animals. This data illustrated there was no detectable skewing of the immune response away from a standard Th2 phenotype associated with immunity to $N$. brasiliensis infection. 
IL-4

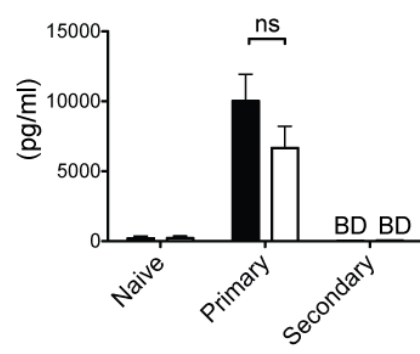

IL-9

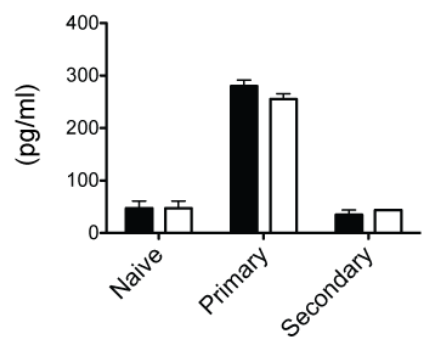

IL-13

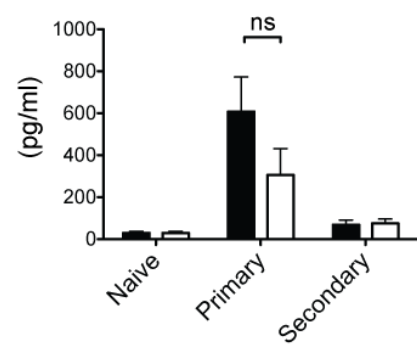

IL-17

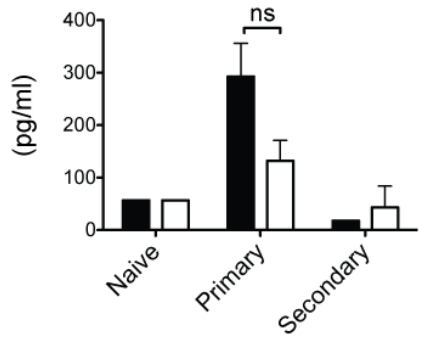

IL-5

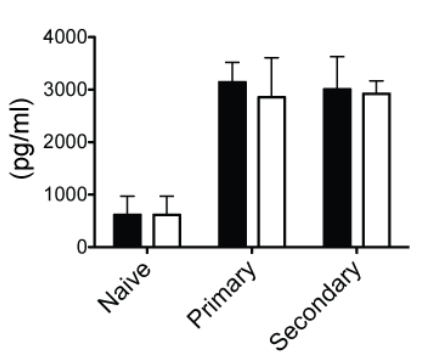

IF $\gamma$

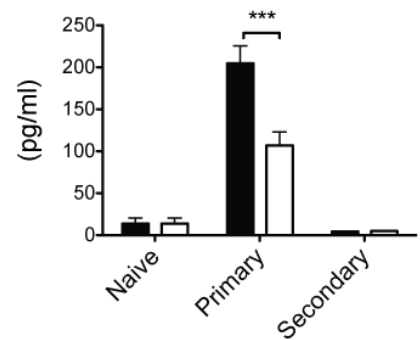

Figure 4. 3 Loss of IL-25 signalling has no effect on type $\mathbf{2}$ cytokines investigated in the lungs following $\boldsymbol{N}$. brasiliensis infection. Lungs from day 9 primary and secondary $N$. brasiliensis infected IL- $25^{+/+}$and IL- $25^{-/-}$mice were processed and MACS sorted for CD45 $5^{+}$cells. The CD $45^{+}$ lung cells were restimulated on $\alpha C D 3 / \alpha C D 28$ beads for $24 \mathrm{hrs}$ in presence of $100 \mathrm{U} / \mathrm{ml} \mathrm{IL-} 2$. Supernatant was assayed using bioplex analysis for cytokines and chemokines. $* p \leq 0.01$, ${ }^{* *} \mathrm{p} \leq 0.001, * * * \mathrm{p} \leq 0.0001 ; 2 \mathrm{Way}$ ANOVA with a Bonferroni post test as compared to IL-25 $5^{+/+}$ animals. Bar graphs show mean $\pm S E M, n \geq 3$ mice/group. BD: Below detection. ns: not significant.

\subsection{Isotype switching to IgE is unaffected by loss of IL-25}

The isotype switching of B cells to IgE requires IL-4 and CD4 T cells ${ }^{205,212}$. Serum $\operatorname{IgE}$ titres serve as a good indication of the functional properties of the Th2 cell, which are thought to provide class-switch signals in the form of CD40 ligation and IL-4 ${ }^{109-}$ ${ }^{111,117}$. Wild type IL-25 $5^{++}$mice and IL-25 $5^{-/}$mice, were infected with $N$. brasiliensis and peripheral blood was harvested from the lateral tail vein at 14 days after primary infection and 9 days after challenge infection. The absence of IL-25 signalling resulted in significantly higher serum titres of $\operatorname{IgE}$ in both primary and secondary responses in comparison to wild type controls (Figure 4. 4). These data demonstrate that the lymphoid CD4 $\mathrm{T}$ cells are functionally intact in their ability to provide B cell 
Chapter Four: The role of IL-25 in the generation of protective T helper type 2 responses against infection by parasitic helminths.

help for isotype class-switch to IgE. Additionally a function for intact IL-25 signalling in repressing IgE class-switch is also evident.
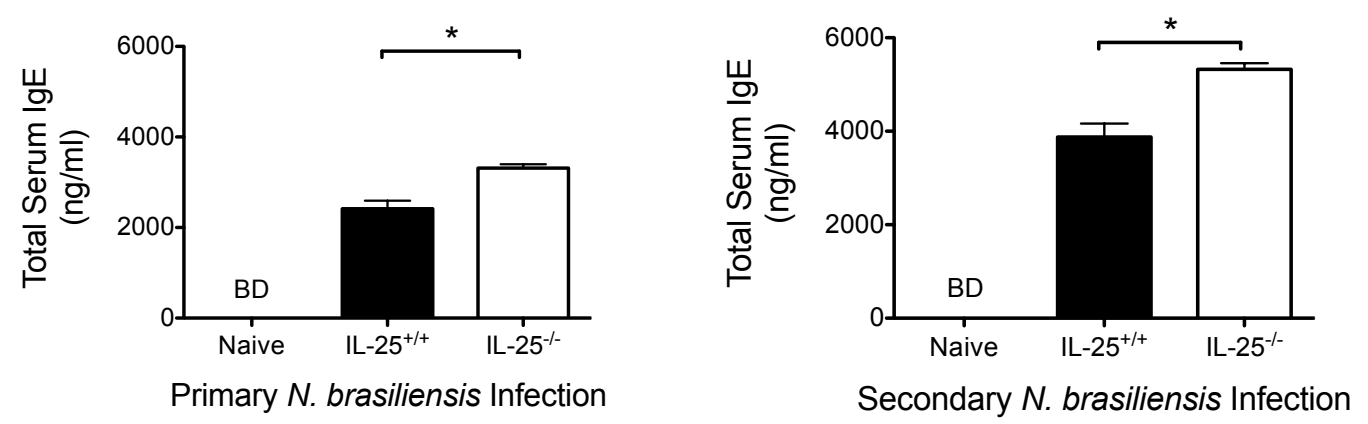

Figure 4. 4 Serum IgE titres are increased in mice deficient in IL-25 signalling. ELISA determined total IgE titres from serum samples obtained at 14 days following primary and 9 days following secondary $N$. brasiliensis infection of $\mathrm{IL}-25^{+/+}$and $\mathrm{IL}-25^{-/-}$mice. $* \mathrm{p} \leq 0.01$, ${ }^{* *} \mathrm{p} \leq 0.001,{ }^{* * *} \mathrm{p} \leq 0.0001$; Mann-Whitney Test; Bar graphs show mean \pm SEM and are representative of 2 or more experiments; $n \geq 4$ mice/group. BD: Below Detection Limit.

\subsection{Basophilia, eosinophilia and goblet cell hyperplasia continue unimpeded in the absence of IL-25 signalling}

The in vivo administration of recombinant IL-25 results in exacerbated type 2 responses ${ }^{137,138,196}$. While IL-4 is the signature cytokine of a Th2 cell, this cell type is not exclusively responsible for its production. Other cell types secreting IL-4 include iNK T cells, basophils, mast cells and eosinophils ${ }^{190}$. In fact, during primary $N$. brasiliensis infections basophils are the major cell type responsible for production of this cytokine in the lung ${ }^{55}$. Basophils reportedly produce not only IL-4 but also IL-25 and are often depicted along with eosinophils as prominent sources of IL-25 ${ }^{147}$. To investigate whether the loss of IL-25 would affect the helminth associated basophilia and eosinophilia; numbers of basophils and eosinophils were determined. 
Basophils were quantified on day 8 in the blood following $N$. brasiliensis primary or secondary infection. The enumeration of blood basophils following primary infection in the IL-25 deficient or IL-4 and IL-25 double deficient animals compared to wild type controls showed no significant differences (Figure 4. 5A). In contrast, at day 8 following secondary infection there was a significant increase in both the frequency and numbers of basophils in the absence of IL-25 (Figure 4. 5B). On a single cell level the ability of the basophils to produce IL-4, as determined by median fluorescent intensity (MdFI) of GFP, was not impacted in the absence of IL-25 signalling (Figure 4. 5A\&B). In summary, the loss of IL-25 signalling resulted in an increase in blood basophils in response to $N$. brasiliensis infection. 
Chapter Four: The role of IL-25 in the generation of protective T helper type 2 responses against infection by parasitic helminths.

\section{A: Primary infection}

(i). Frequency of $\mathrm{GFP}^{+}$basophils

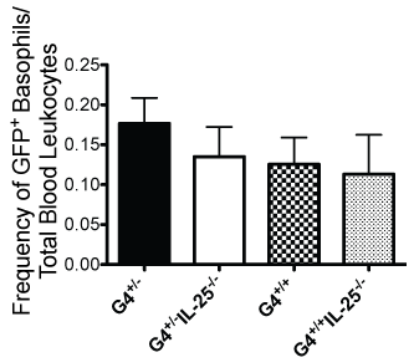

Primary N. brasiliensis infection (ii). Numbers of $\mathrm{GFP}^{+}$basophils

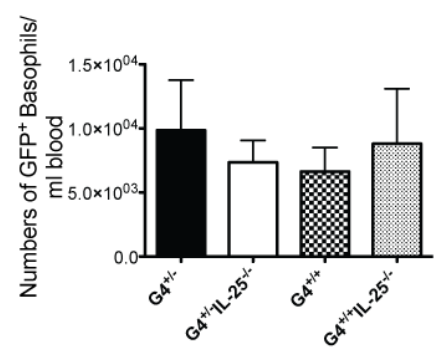

Primary N. brasiliensis infection (iii). MdFI of $\mathrm{GFP}^{+}$basophils

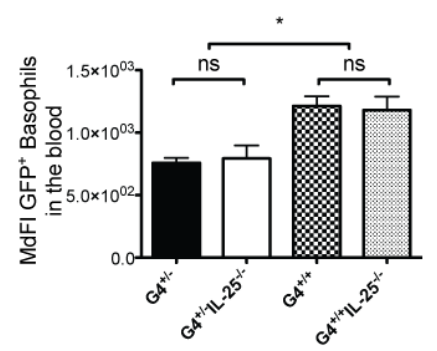

Primary N. brasiliensis infection

\section{B: Secondary infection}

(i). Frequency of $\mathrm{GFP}^{+}$basophils

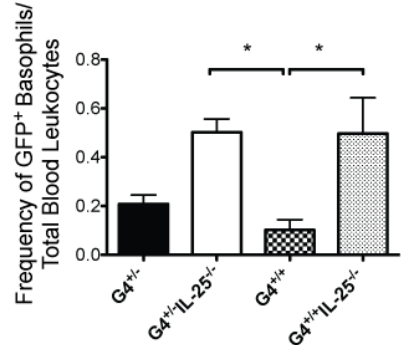

Secondary $N$. brasiliensis infection (ii). Numbers of $\mathrm{GFP}^{+}$basophils

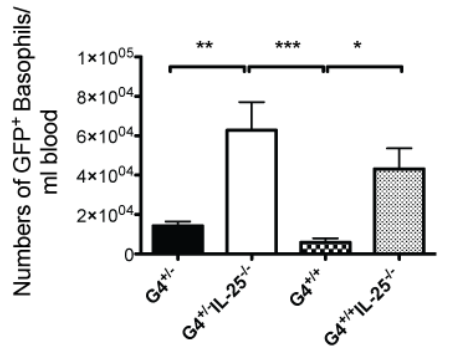

Secondary $N$. brasiliensis infection (iii). MdFI of $\mathrm{GFP}^{+}$basophils

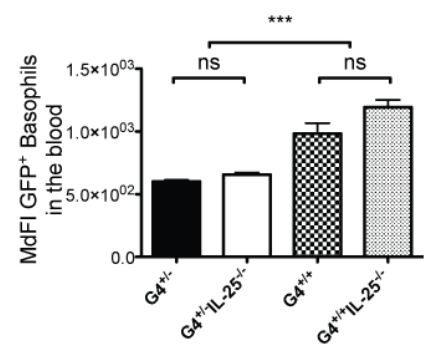

Secondary N. brasiliensis infection

Figure 4. 5 IL-25 deficiency results in increased blood basophilia following secondary $\mathbf{N}$. brasiliensis infections. Basophil (Doublet live Non-T Non-B cell CD16/32 ${ }^{+}{\text {CD } 45^{\text {int }}}^{-}$FceR $1 \alpha^{+}$

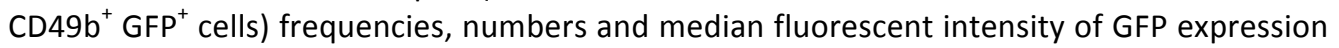
were determined by flow cytometry at A: day 8 following primary infection with $N$. brasiliensis B: day 8 following secondary infection with $N$. brasiliensis. ${ }^{*} p \leq 0.01,{ }^{* *} p \leq 0.001$, ${ }^{* * *} \mathrm{p} \leq 0.0001$; 1 Way ANOVA with a Tukey post test; Bar graphs show mean \pm SEM and are representative of 2 or more experiments; $n \geq 5$ mice/group. ns: not significant.

Total numbers of eosinophils and IL-4 producing $\mathrm{GFP}^{+}$eosinophils were enumerated per millilitre of blood at day 8 following primary $N$. brasiliensis infection. The primary response indicated no significant differences between IL-25 deficient animals and their wild type controls, although there was a trend towards increased IL-4 producing eosinophils in the absence of IL-25 (Figure 4. 6A). This trend became a significant difference in the secondary response to $N$. brasiliensis, with significantly increased numbers of both total eosinophils and IL-4 producing eosinophils at day 8 after secondary infection with $N$. brasiliensis in IL-25 deficient animals (Figure 4. 
6B). In summary, the absence of IL-25 resulted in increased blood eosinophils following secondary infection with $N$. brasiliensis.

\section{A: Primary infection}

(i). Numbers of eosinophils

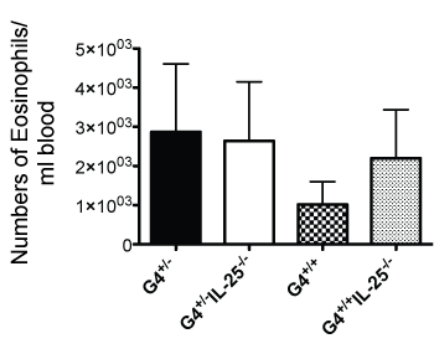

Primary N. brasiliensis infection (ii). Numbers of $\mathrm{GFP}^{+}$eosinophils

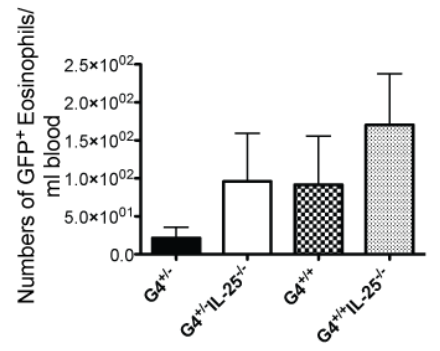

Primary N. brasiliensis infection (iii). MdFI of $\mathrm{GFP}^{+}$eosinophils

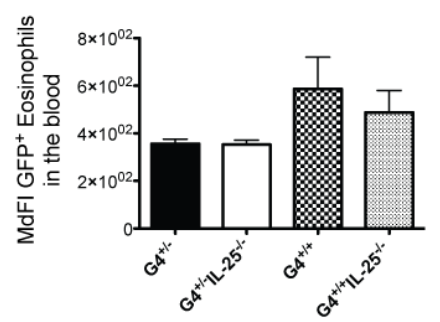

Primary N. brasiliensis infection

\section{B: Secondary infection}

(i). Numbers of eosinophils

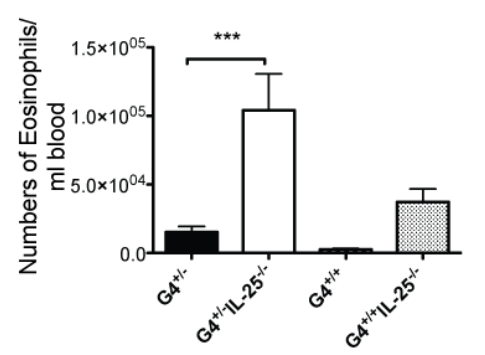

Secondary $N$. brasiliensis infection (ii). Numbers of GFP+ ${ }^{+}$eosinophils

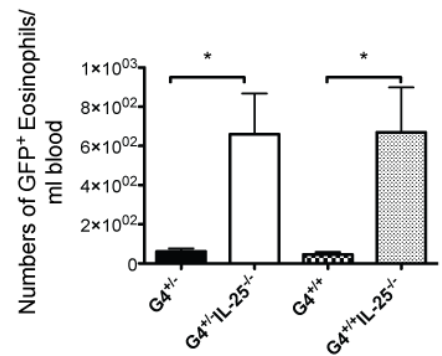

Secondary N. brasiliensis infection (iii). MdFI of $\mathrm{GFP}^{+}$eosinophils

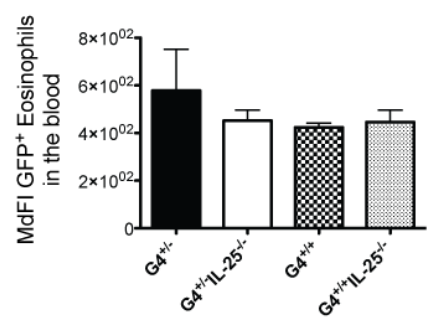

Secondary $N$. brasiliensis infection

Figure 4. 6 A lack of IL-25 signalling resulted in increased blood eosinophilia following secondary $\boldsymbol{N}$. brasiliensis infections. IL-4 producing eosinophils were determined by the

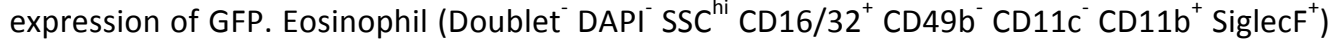
frequencies, numbers and median fluorescent intensity of GFP expression were determined by flow cytometry at $\underline{\text { A: }}$ day 8 following primary infection with $N$. brasiliensis $\underline{\text { B: }}$ day 8 following secondary infection with $N$. brasiliensis. ${ }^{*} p \leq 0.01,{ }^{*} p \leq 0.001,{ }^{* * *} \mathrm{p} \leq 0.0001$; 1 Way ANOVA with a Tukey post test; Bar graphs show mean \pm SEM and are representative of 2 or more experiments; $n \geq 5$ mice/group.

A lymphocyte orchestrated adaptive immune response is required for recruitment and expansion of innate effector cells, such as eosinophils, in the lung tissue following infection with $N$. brasiliensis infection ${ }^{213}$. The intent of this section was to investigate 
Chapter Four: The role of IL-25 in the generation of protective T helper type 2 responses against infection by parasitic helminths.

whether production of the innate cytokine IL-25 had any effect on the recruitment of eosinophils into the lung tissues. $\mathrm{G}^{+/-}, \mathrm{G} 4^{+/} \mathrm{IL}-25^{-/}, \mathrm{G} 4^{+/+}$and $\mathrm{G} 4^{+/+} \mathrm{IL}_{-2}-25^{-/}$mice were infected subcutaneously with 600 L3 N. brasiliensis and lung tissues were perfused and harvested 9 days later. For a memory immune response to the same antigen animals were challenged with 600 L3 larvae and 9 days later lungs were perfused and harvested to enumerate eosinophils. Eosinophils were determined by flow cytometry as $\mathrm{SSC}^{\text {hi }} \mathrm{CD} 16 / 32^{+} \mathrm{CD} 49 \mathrm{~b}^{-} \mathrm{CD} 11 \mathrm{c}^{-} \mathrm{CD}_{11 \mathrm{~b}^{+}}$SiglecF $^{+}$cells and enumerated as total cells per lung or for IL-4 producing eosinophils as total $\mathrm{GFP}^{+}$eosinophils per lung. The lack of IL-4 significantly reduced the total numbers of eosinophils recruited into the lungs following both the primary and the secondary challenges (Figure 4.7 A\&B). The lack of IL-25 signalling, however, resulted in no defects in recruitment of either total eosinophils or IL-4 producing eosinophils into the lung tissues following both primary and secondary infection (Figure 4.7 A\&B). In summary, these data demonstrate no apparent role for IL-25 in recruitment of eosinophils into lung tissues following $N$. brasiliensis infection.

Lastly, mucous production by goblet cells was investigated in mice deficient in IL-25 because this is a good indication of biologically active IL-13 ${ }^{168,214}$. Primary and secondary day $9 \mathrm{~N}$. brasiliensis infected lung tissues were processed and stained with Haemotoxylin and Periodic Acid-Schiff (PAS) for the comparison of mucous production by goblet cells lining the bronchioles. Remarkably, in the absence of IL25, if anything, a trend towards increased mucous production was noted (Figure 4. 8), indicating that a lack of IL-25 resulted in no significant deficiency in this IL-13 mediated effector response. 
Chapter Four: The role of IL-25 in the generation of protective T helper type 2 responses against infection by parasitic helminths.

\section{A: Primary infection}

(i). Numbers of eosinophils

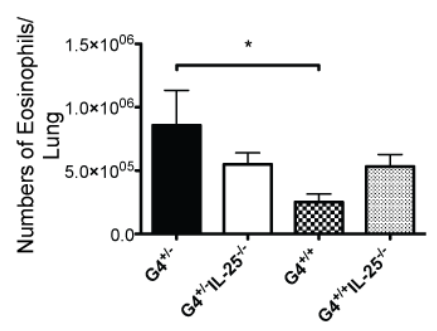

Primary N. brasiliensis infection (ii). Numbers of GFP+ eosinophils

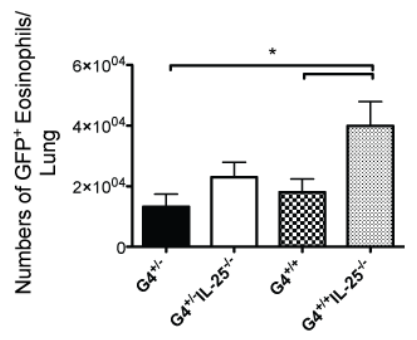

Primary N. brasiliensis infection (iii). MdFI of $\mathrm{GFP}^{+}$eosinophils

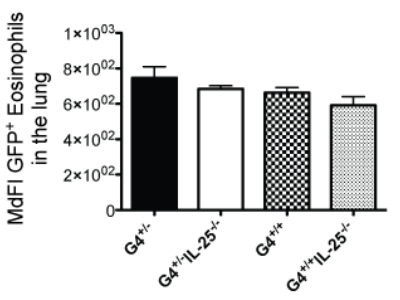

Primary N. brasiliensis infection

\section{B: Secondary infection}

(i). Numbers of eosinophils

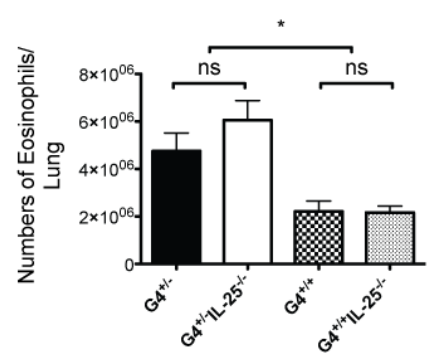

Secondary N. brasiliensis infection (ii). Numbers of GFP+ eosinophils

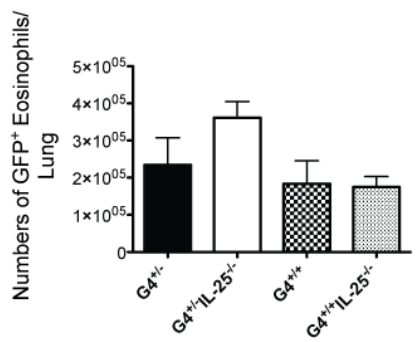

Secondary $N$. brasiliensis infection (iii). MdFI of GFP+ eosinophils

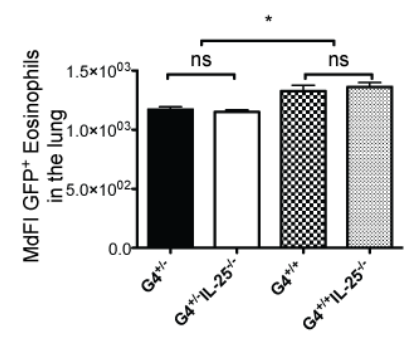

Secondary N. brasiliensis infection

Figure 4. 7 A lack of IL-25 signalling had little effect on eosinophilia in the lungs following secondary $N$. brasiliensis infections. Eosinophil (Doublet ${ }^{-} \mathrm{DAPI}^{-} \mathrm{SSC}^{\mathrm{hi}} \mathrm{CD} 16 / 32^{+} \mathrm{CD} \mathrm{Cb}^{-} \mathrm{CD} 11 \mathrm{c}^{-}$ $\mathrm{CD}_{11 \mathrm{~b}^{+}}$SiglecF $^{+}$) numbers, the expression of $\mathrm{IL}-4$ as reported by GFP and the median fluorescent intensity (MdFI) of GFP were determined by flow cytometry at $\underline{A}$ : day 9 following primary infection with $N$. brasiliensis B: day 9 following secondary infection with $N$. brasiliensis. ${ }^{*} p \leq 0.01,{ }^{*} p \leq 0.001,{ }^{* * *} \mathrm{p} \leq 0.0001$; 1 Way ANOVA with a Tukey post test; Bar graphs show mean $\pm S E M$ and are representative of 2 or more experiments; $n \geq 5$ mice/group. ns: not significant. 


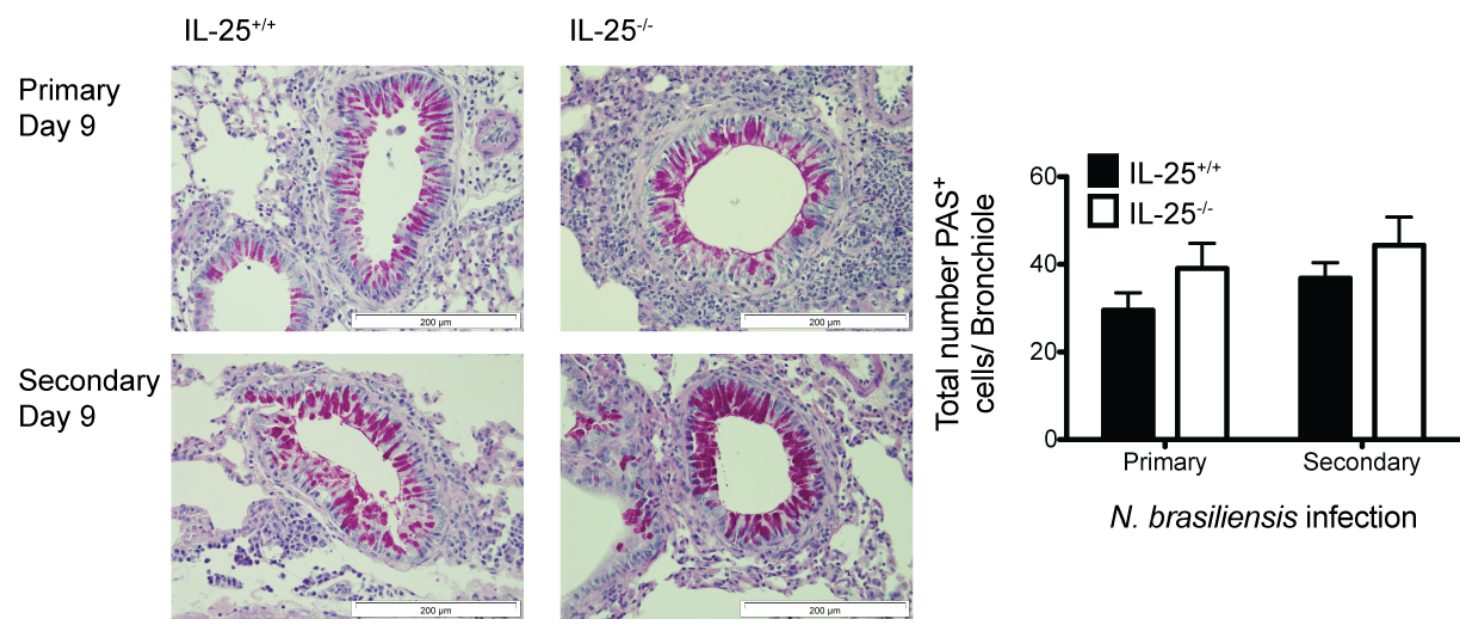

Figure 4. 8 IL-25 signalling is not required for production of lung mucous following infection with $\boldsymbol{N}$. brasiliensis. Lung sections from day 9 primary and secondary $N$. brasiliensis infected mice were processed and stained with Mayer's Haematoxylin and Periodic Acid Schiff (PAS). Total numbers of $\mathrm{PAS}^{+}$cells per bronchiole were quantified and graphically represented. ${ }^{*} p \leq 0.01,{ }^{*} \mathrm{p} \leq 0.001,{ }^{* *} \mathrm{p} \leq 0.0001 ; 1$ Way ANOVA with a Tukey post test; Bar graphs show mean $\pm S E M$ and are representative of 2 or more experiments; $n \geq 5$ mice/group.

\subsection{Loss of IL-25 has no effect on the nuocyte populations detectable in lungs or mediastinal lymph nodes}

Recent investigations demonstrate IL-13 producing lineage negative innate lymphoid cells, denoted nuocytes, are mediators of immunity necessary for gut expulsion of parasitic helminths and demonstrate these cells are capable of inducing airway hyperresponsiveness in allergic airways inflammation models ${ }^{61,211}$. The administration of rIL-25 results in expansion of these cells in the airways, lymph nodes and the peritoneal cavity ${ }^{175,211}$, while animals lacking the IL-25 receptor exibit a delayed expansion of nuocytes in mesenteric lymph nodes in response to parasitic infection ${ }^{61}$. Nuocytes are described as resembling lymphocytes yet lacking all lineage markers associated with haematopoietic cells. Reports vary on expression levels of key extracellular markers including Sca-1 and c-Kit 175,215,216. However nuocytes do consistently express IL-7R $\alpha$ and ICOS. In this thesis, nuocytes were determined by multicolour flow cytometry by excluding doublets and dead cells then selecting a 
lymphocyte gate with SSC low cells that were $\mathrm{CD}^{+} 5^{+}$but lineage negative $(\mathrm{CD} 3$, B220, CD11b, FceR1 $\alpha$, CD4, CD8, CD11c, GR1, CD49b), Sca-1, IL-7R $\alpha$ and ICOS positive. c-Kit was excluded from the description of these cells, as the expression levels were negligible (Figure 4.9). To determine IL-4 expression, nuocytes were investigated for the expression of GFP and enumerated as total numbers of GFP producing nuocytes per organ. Figure 4.9 illustrates the identification and characterisation of nuocytes in the mediastinal lymph nodes and this is representative of gating strategies used for identification of nuocytes in both the lungs and mesenteric lymph nodes in subsequent figures.
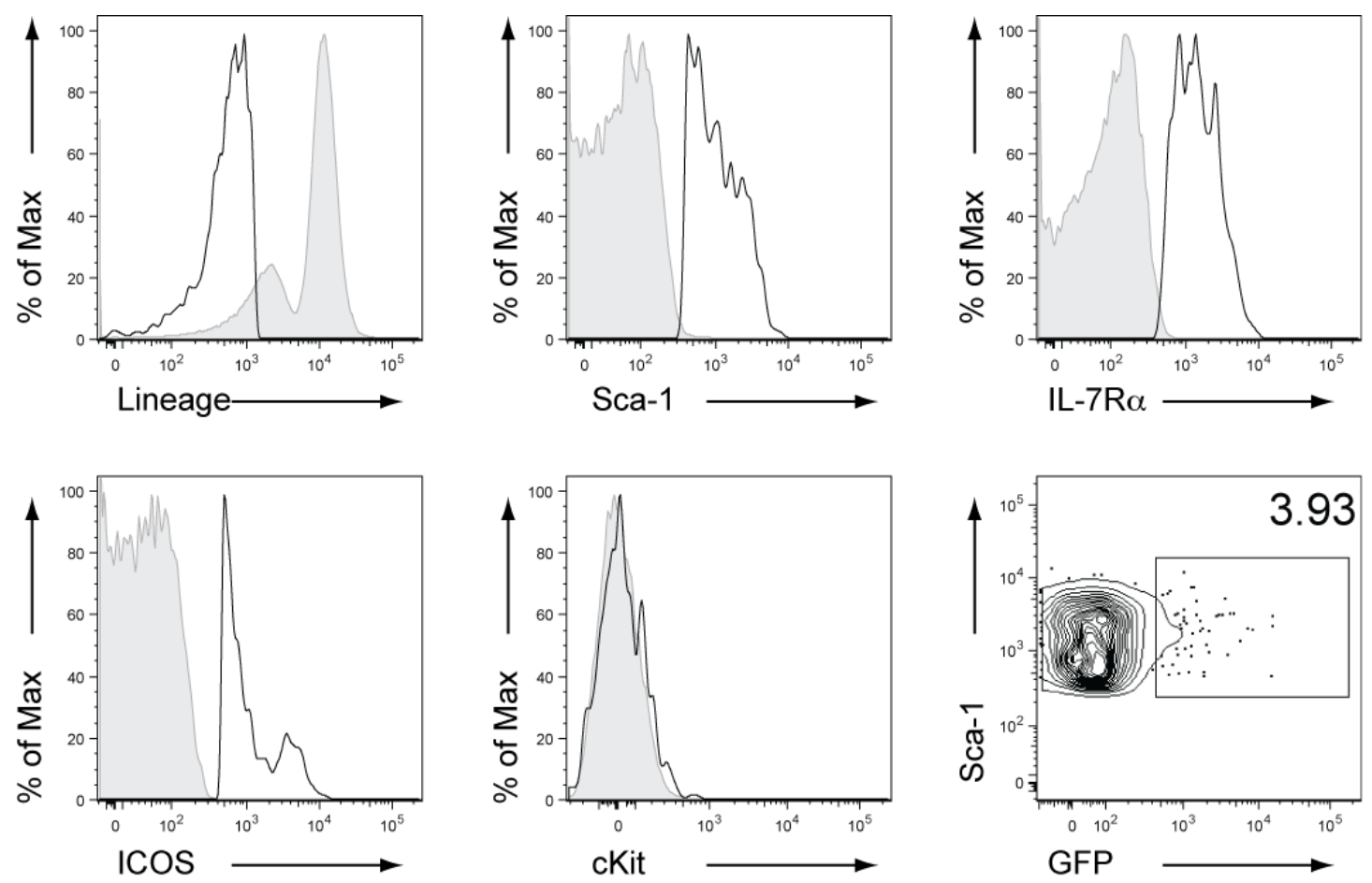

Figure 4. 9 Identification and characterisation of nuocytes in the lung, mediastinal and mesenteric lymph nodes following $\boldsymbol{N}$. brasiliensis infection. Mediastinal lymph nodes from day 9 secondary infected mice were processed and stained for multicolour flow cytometric analysis. Nuocytes were determined as doublet ${ }^{-} \mathrm{DAPI}^{-} \mathrm{SSC}^{\mathrm{lo}} \mathrm{CD}_{4} 5^{+}$Lineage $^{-} \mathrm{Sca}^{+} \mathrm{IL}_{-} 7 \mathrm{R}^{+}$ $\mathrm{ICOS}^{+} \mathrm{c}$-Kit'. Open lines represent nuocytes overlaid onto grey filled lines representing isotype controls (except for lineage plot that represents all live cells in the sample).

To determine whether nuocytes were present in the lungs and the mediastinal lymph node of mice infected with $N$. brasiliensis, the frequency and total number of IL-4 
Chapter Four: The role of IL-25 in the generation of protective T helper type 2 responses against infection by parasitic helminths.

producing nuocytes were determined over the course of the primary and secondary immune responses. $\mathrm{G} 4^{+/-}, \mathrm{G}^{+/-} \mathrm{IL}-25^{-/}, \mathrm{G}^{+/+}, \mathrm{G} 4^{+/+} \mathrm{IL}^{-25^{-/}}$animals were infected with 600 L3 N. brasiliensis, 45 days later these same animals were challenged with the same number of parasites to induce a secondary immune response. Flow cytometry was used to determined the frequency of nuocytes in naïve animals and on days 3, 6 and 9 following primary infection, following 45 days rest and then subsequently 3,6 and 9 days after re-infection. In the absence of IL-25 and IL-4 there was no defect in nuocyte population expansion in either the lymph nodes or lungs following primary and secondary infections (Figure 4. 10, Figure 4. 11, Figure 4. $12 \&$ Figure 4. 13). The only differences were noted in the lungs of IL-4 sufficient IL-25 deficient animals at day 6 following re-infection with $N$. brasiliensis where the numbers of nuocytes were significantly less in the absence of IL-25. However by day 9 the numbers were similar to the IL-25 wild type control once again (Figure 4. 12). This did not occur in the lungs of animals lacking signalling from both IL-25 and IL-4 (Figure 4. 13). Taken together these data demonstrate that the absence of IL-25 had no effect on the influx or expansion of nuocytes to the lung or mediastinal lymph node following primary or secondary N. brasiliensis infection.

As IL-4 is required for the generation of protective immunity against $N$. brasiliensis it was decided to investigate whether these newly described nuocytes were contributing large quantities of IL-4 to the immune response. Total numbers of $\mathrm{GFP}^{+}$nuocytes were compared to total numbers of $\mathrm{GFP}^{+} \mathrm{Th} 2$ cells in the mediastinal lymph nodes and lungs following primary and secondary $N$. brasiliensis infection. IL-4 producing Th2 cells significantly outnumbered the IL-4 producing nuocytes (Figure 4. 14A). The total number of CD4 T cells significantly outnumbered the total numbers of nuocytes in both the mediastinal lymph node and lung during both the primary and secondary immune response to $N$. brasiliensis infection (Figure 4. 14B). In summary, in the lymph nodes and lungs of an $N$. brasiliensis infected animals the Th2 cells constitute the greater proportion of IL-4 producing cells in comparison to the nuocyte population. 


\section{A: IL-4 Sufficient Mediastinal Lymph Node}

(i). Frequency of nuocytes

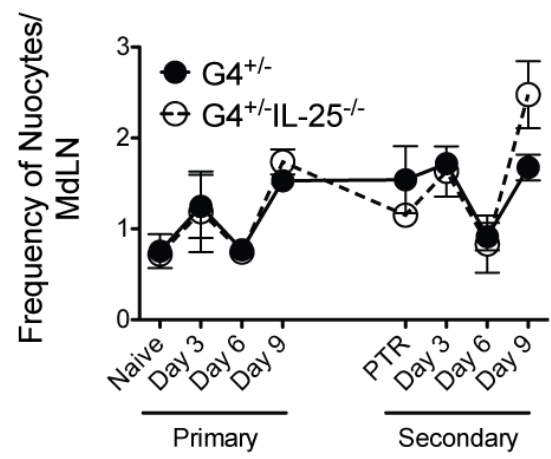

N. brasiliensis infection (ii). Numbers of nuocytes

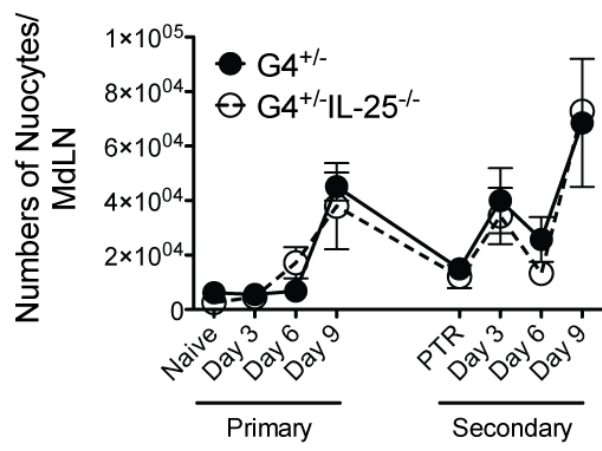

N. brasiliensis infection

\section{B: IL-4 Sufficient Mediastinal Lymph Node}

(i). Frequency of GFP+ nuocytes

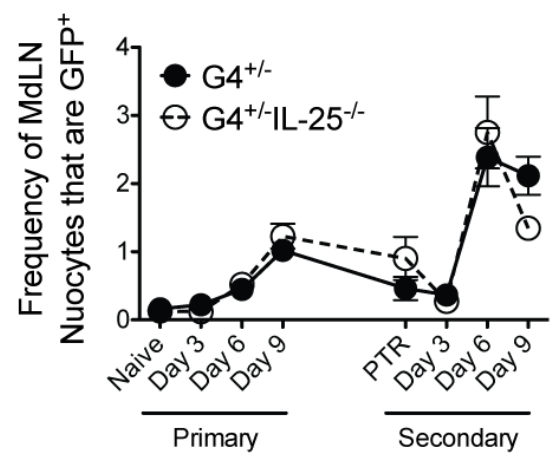

N. brasiliensis infection (ii). Numbers of GFP+ nuocytes

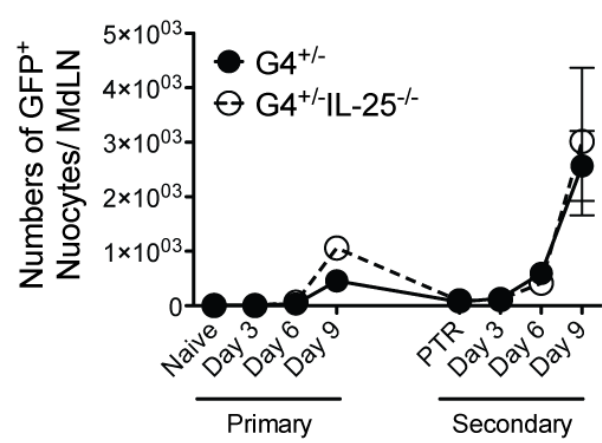

N. brasiliensis infection

Figure 4. 10 IL-25 is not required for expansion of the nuocyte population in the mediastinal lymph node following $\boldsymbol{N}$. brasiliensis infection. Mediastinal lymph nodes (MdLN) were harvested 3, 6 and 9 days following primary infection with 600 L3 SQ N. brasiliensis. Mice were challenged at 45 days after primary infection and MdLNs were harvested 3, 6 and 9 days later to determine secondary immune responses. MdLNs were processed into single cell suspensions, stained and analysed by flow for $\underline{A}$ : frequency of Nuocytes (doublet DAPI SSC ${ }^{\circ}$ $\mathrm{CD} 5^{+}$Lineage $\mathrm{Sca}^{+} \mathrm{IL}^{-7 \mathrm{Ra}^{+}} \mathrm{ICOS}^{+}$) that were enumerated as total nuocytes per lymph node and B: frequency of IL-4 producing $\mathrm{GFP}^{+}$Nuocytes that were enumerated as total $\mathrm{GFP}^{+}$ nuocytes per lymph node ${ }^{*} p \leq 0.01, * * p \leq 0.001, * * * p \leq 0.0001 ; 2$ Way ANOVA with a Bonferroni post test as compared to $\mathrm{G}^{+/-}$. Line graphs show mean $\pm \mathrm{SEM}, \mathrm{n} \geq 4$ mice/group. PTR: Prior to re-infection. 
Chapter Four: The role of IL-25 in the generation of protective T helper type 2 responses against infection by parasitic helminths.

\section{A: IL-4 Deficient Mediastinal Lymph Node}

(i). Frequency of nuocytes

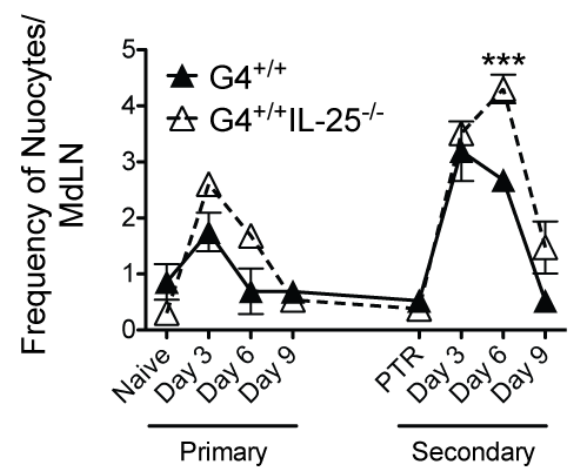

N. brasiliensis infection (ii). Numbers of nuocytes

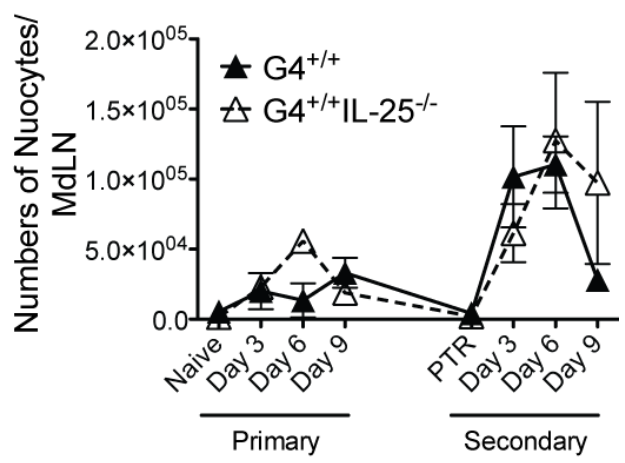

N. brasiliensis infection

\section{B: IL-4 Deficient Mediastinal Lymph Node}

(i). Frequency of $\mathrm{GFP}^{+}$nuocytes

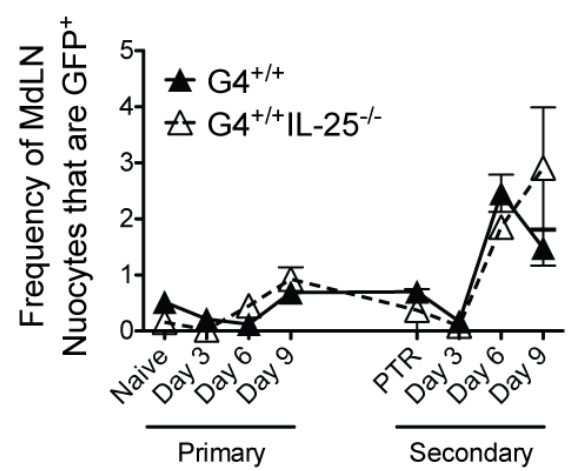

N. brasiliensis infection (ii). Numbers of GFP+ nuocytes

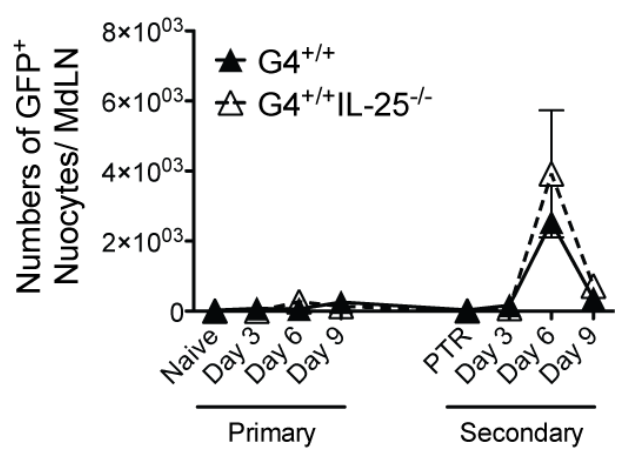

N. brasiliensis infection

Figure 4. 11 IL-25 is not required for nuocytes expansion in the mediastinal lymph node following $\boldsymbol{N}$. brasiliensis infection in the absence of IL-4. Mediastinal lymph nodes (MdLN) were harvested 3, 6 and 9 days following primary infection with 600 L3 SQ N. brasiliensis. Mice were challenged at 45 days after primary infection and MdLNs were harvested 3, 6 and 9 days later to determine secondary immune responses. MdLNs were processed into single cell suspensions, stained and analysed by flow for $\underline{\mathrm{A}}$ : frequency of Nuocytes (doublet ${ }^{-} \mathrm{DAPI}^{-}$ $\mathrm{SSC}^{\text {lo }} \mathrm{CD} 5^{+}$Lineage $\mathrm{Sca}^{+} \mathrm{IL}_{-7 \mathrm{Ra}^{+}} \mathrm{ICOS}^{+}$) that were enumerated as total nuocytes per lymph node in B: frequency of IL-4 producing GFP Nuocytes and enumerated as total GFP ${ }^{+}$nuocytes per lymph node ${ }^{*} \mathrm{p} \leq 0.01,{ }^{* *} \mathrm{p} \leq 0.001,{ }^{* *} \mathrm{p} \leq 0.0001 ; 2$ Way ANOVA with a Bonferroni post test as compared to $\mathrm{G}^{+/+}$. Line graphs show mean $\pm \mathrm{SEM}, \mathrm{n} \geq 4$ mice/group. PTR: Prior to reinfection. 


\section{A: IL-4 Sufficient Lung}

(i). Frequency of nuocytes

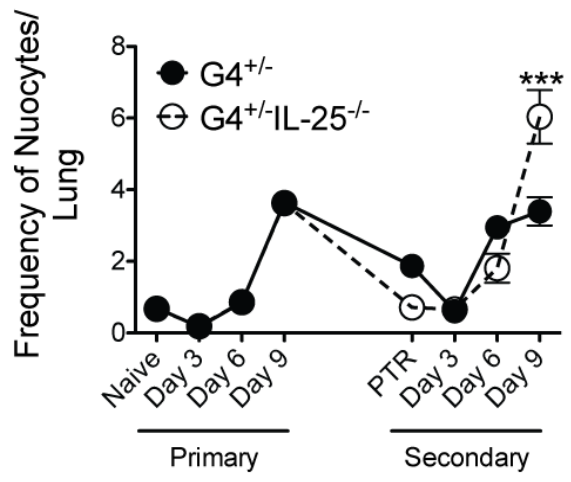

N. brasiliensis infection (ii). Numbers of nuocytes

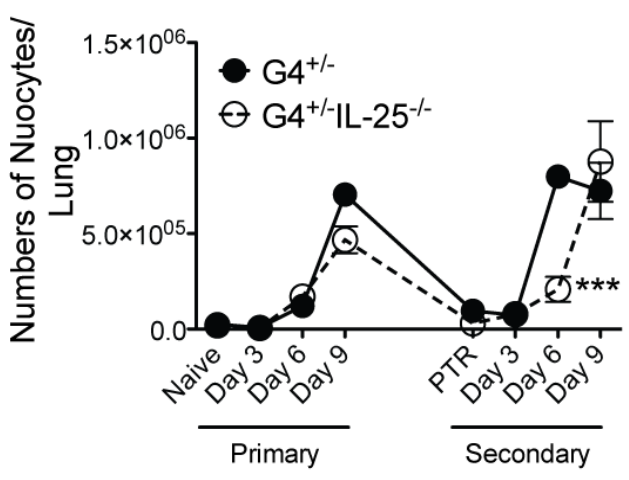

N. brasiliensis infection

\section{B: IL-4 Sufficient Lung}

(i). Frequency of $\mathrm{GFP}^{+}$nuocytes

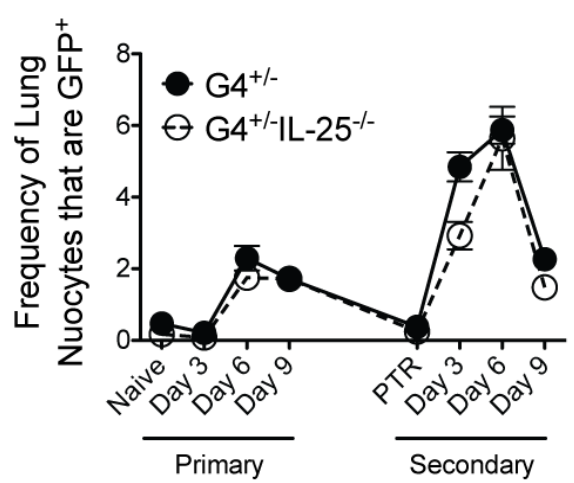

N. brasiliensis infection (ii). Numbers of $\mathrm{GFP}^{+}$nuocytes

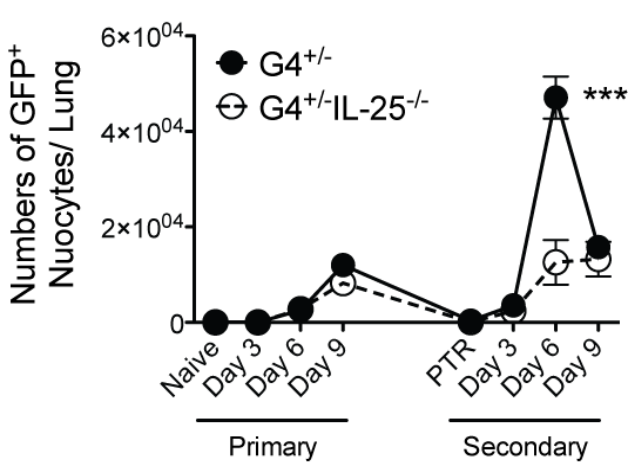

N. brasiliensis infection

Figure 4. 12 IL-25 is not required for nuocytes expansion in the lung following $\mathbf{N}$. brasiliensis infection. Lungs were harvested 3, 6 and 9 days following primary infection with 600 L3 SQ N. brasiliensis. Mice were challenged at 45 days after primary infection and lungs were harvested 3, 6 and 9 days later to determine secondary immune responses. Lungs were processed into single cell suspensions, stained and analysed by flow for $\underline{A}$ : frequency of Nuocytes (doublet DAPI SSC ${ }^{10} \mathrm{CD}^{-} 5^{+}$Lineage $^{-} \mathrm{Sca}^{+}{ }^{+} \mathrm{IL}-7 \mathrm{Ra}^{+} \mathrm{ICOS}^{+}$) that were enumerated as total nuocytes per lymph node in B: frequency of IL-4 producing GFP ${ }^{+}$Nuocytes and enumerated as total GFP ${ }^{+}$nuocytes per lymph node ${ }^{*} p \leq 0.01, * * p \leq 0.001, * * * p \leq 0.0001 ; 2$ Way ANOVA with a Bonferroni post test as compared to $\mathrm{G}^{+/-}$. Line graphs show mean $\pm \mathrm{SEM}, \mathrm{n} \geq 4$ mice/group. PTR: Prior to re-infection. 
Chapter Four: The role of IL-25 in the generation of protective T helper type 2 responses against infection by parasitic helminths.

\section{A: IL-4 Deficient Lung}

(i). Frequency of nuocytes

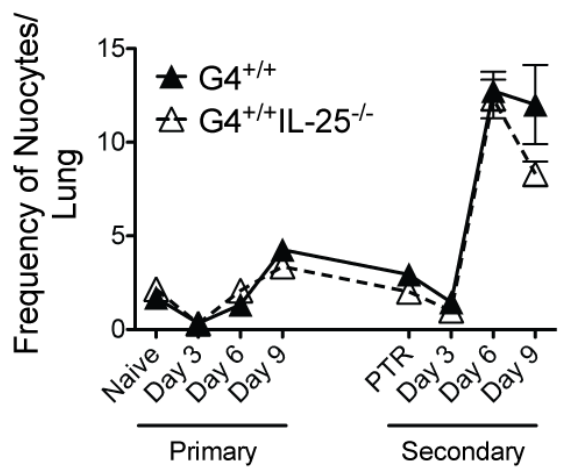

N. brasiliensis infection (ii). Numbers of nuocytes

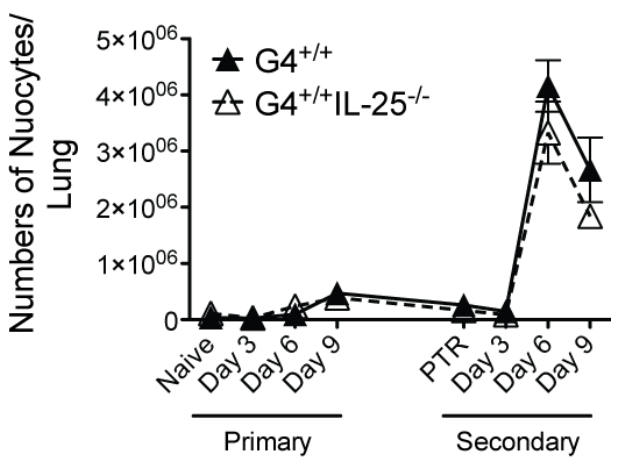

N. brasiliensis infection

\section{B: IL-4 Deficient Lung}

(i). Frequency of $\mathrm{GFP}^{+}$nuocytes

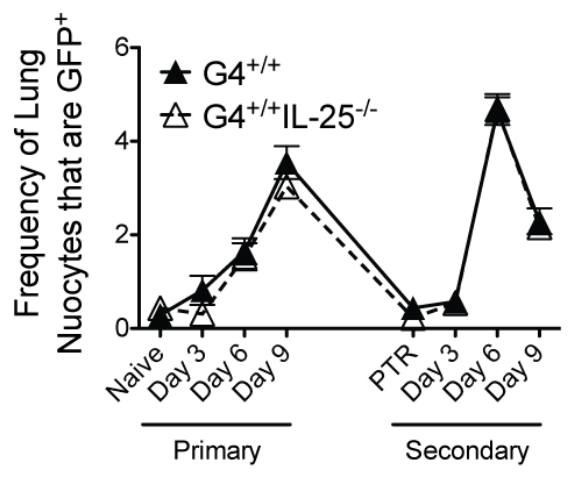

N. brasiliensis infection (ii). Numbers of $\mathrm{GFP}^{+}$nuocytes

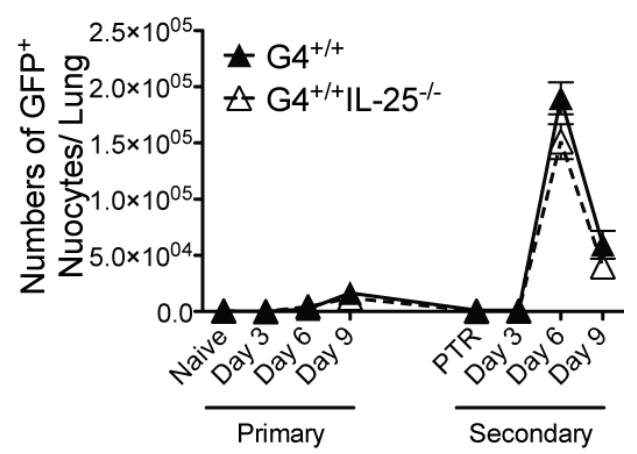

N. brasiliensis infection

Figure 4. 13 Even in the absence of IL-4, IL-25 is not required for nuocytes expansion in the lungs following $\boldsymbol{N}$. brasiliensis infection. Lungs were harvested 3, 6 and 9 days following primary infection with 600 L3 SQ N. brasiliensis. Mice were challenged at 45 days after primary infection and lungs were harvested 3, 6 and 9 days later to determine secondary immune responses. Lungs were processed into single cell suspensions, stained and analysed by flow for $\underline{\mathbf{A}}$ : frequency of Nuocytes (doublet ${ }^{-} \mathrm{DAPI}^{-} \mathrm{SSC}^{\mathrm{lo}}$ Lineage $^{-} \mathrm{Sca}^{+}{ }^{+} \mathrm{IL}-7 \mathrm{Ra}^{+} \mathrm{ICOS}^{+}$) that were enumerated as total nuocytes per lymph node in B: frequency of IL-4 producing GFP ${ }^{+}$ Nuocytes and enumerated as total $\mathrm{GFP}^{+}$nuocytes per lymph node ${ }^{*} p \leq 0.01,{ }^{* *} p \leq 0.001$, $* * * \mathrm{p} \leq 0.0001 ; 2$ Way ANOVA with a Bonferroni post test as compared to $\mathrm{G}^{+/+}$, Line graphs show mean $\pm S E M, n \geq 4$ mice/group. PTR: Prior to re-infection. 
Chapter Four: The role of IL-25 in the generation of protective T helper type 2 responses against infection by parasitic helminths.

\section{A: Comparison of IL-4 producing cells}

(i). Mediastinal Lymph Node

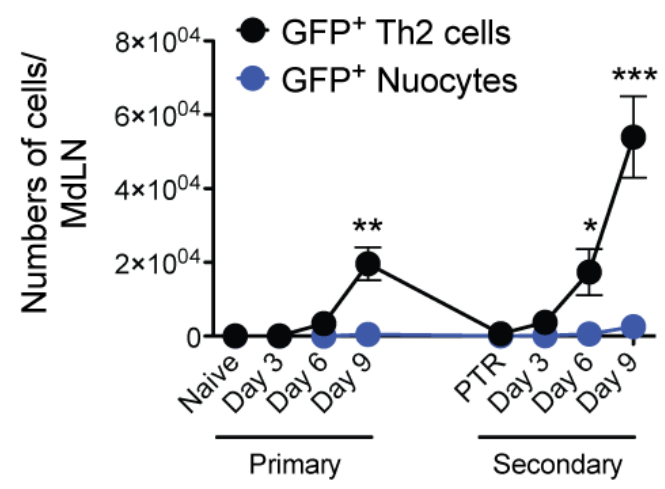

N. brasiliensis infection (ii). Lung

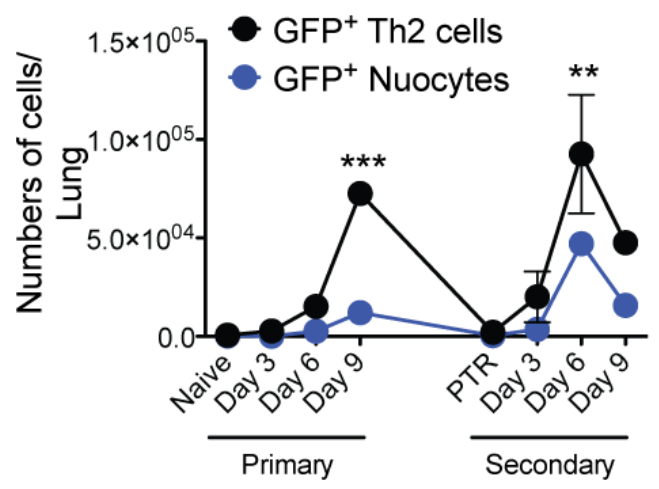

N. brasiliensis infection

\section{B: Comparison of total cells}

(i). Mediastinal Lymph Node

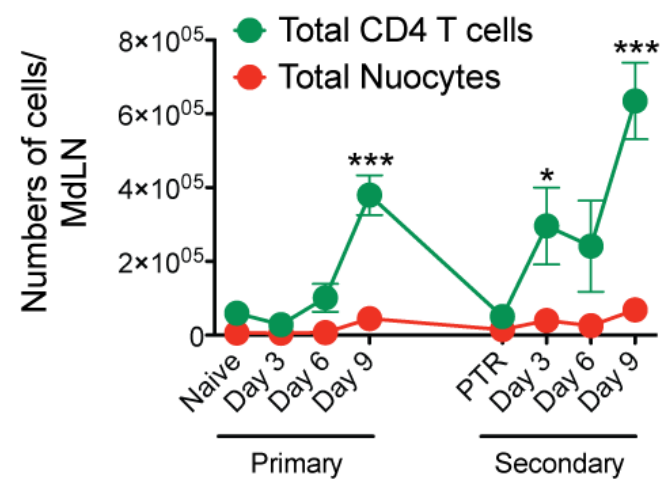

N. brasiliensis infection (ii). Lung

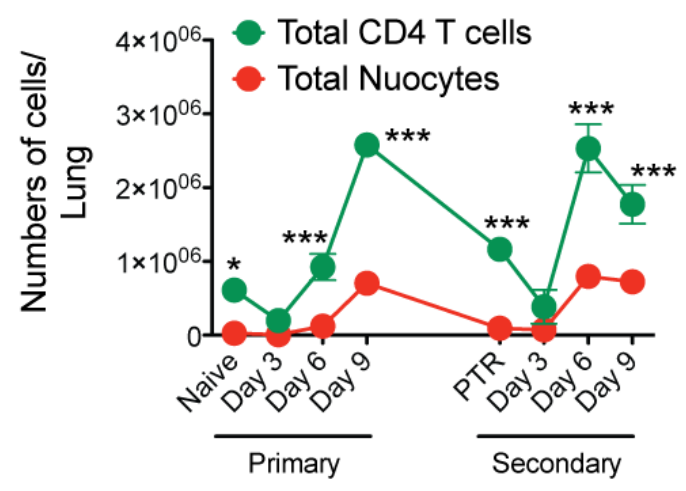

N. brasiliensis infection

Figure 4. 14 Th2 cells rather than nuocytes are the predominant source of IL-4 in the mediastinal lymph node and lung during $\boldsymbol{N}$. brasiliensis infection. Lungs and mediastinal lymph nodes (MdLN) were harvested 3, 6 and 9 days following primary infection with 600 L3 SQ $N$. brasiliensis. Mice were challenged at 45 days after primary infection and lungs and MdLNs were harvested 3, 6 and 9 days later to determine secondary immune responses. Lungs and MdLNs were processed into single cell suspensions, stained and analysed by flow for numbers of A: IL-4 producing Th2 cells (Doublet DAPI ${ }^{-} \mathrm{CD}^{+} \mathrm{CD}^{+} \mathrm{GFP}^{+}$cells) and $\mathrm{GFP}^{+} \mathrm{IL}-4$

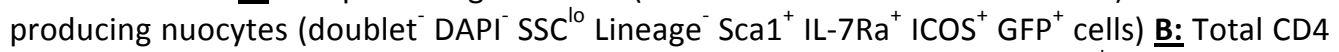
T cells (Doublet ${ }^{-D A P I}{ }^{-} \mathrm{CD}^{+} \mathrm{CD}^{+}$cells) and total nuocytes (doublet ${ }^{-} \mathrm{DAPI}^{-} \mathrm{SSC}^{\mathrm{lo}}$ Lineage $^{-} \mathrm{Sca1}^{+}$

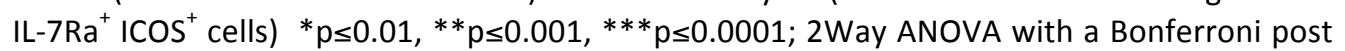
test. Line graphs show mean \pm SEM, $n \geq 4$ mice/group. PTR: Prior to re-infection. 
Chapter Four: The role of IL-25 in the generation of protective T helper type 2 responses against infection by parasitic helminths.

\subsection{Lung protective immunity to $\mathbf{N}$. brasiliensis continues in the absence of IL-25 signalling}

Previous investigations have demonstrated that animals deficient in IL-25 have a delayed ability to expel intestinal $N$. brasiliensis ${ }^{175}$. To further expand on these findings, we performed a kinetics study using IL-25 sufficient and IL-25 deficient animals in which worm counts were measured regularly in the lung and small intestine following a primary (Figure 4.15A) and a secondary infection (Figure 4. 15B) with $N$. brasiliensis. The majority of the parasites passed through the lung by day 2-post infection with little variability between IL-25 deficient animals and the wild type controls (Figure 4. 15A). The highest worm counts in the guts were observed after 4-6 days of infection, followed by a rapid drop in worm numbers with complete expulsion in the wild type by day 9-post infection (Figure 4. 15A). A relatively small but persistent worm population was maintained in the IL-25 deficient animals from day 9 until about day 12 (Figure 4. 15A). This data is consistent with two phases (lung and guts) of $N$. brasiliensis infection.

One of the research objectives of chapter four was to determine whether IL-25 played a role in protective immunity against $N$. brasiliensis. Protective immunity was defined as the significantly decreased worm burden noted in the lungs of the wild type mice, in comparison to their primary infection worm burdens, following a challenge infection. This was noted in the lungs of wild type challenged mice in comparison to the worm burdens seen in those same animals during a primary infection (Figure 4. 15B). Numbers of viable worms recoverable from day 2 lungs dropped off significantly, from approximately 150 worms during the primary to less than 25 during the secondary infection, this is indicative of a faster more efficient immune response preventing the parasites from establishing their usual infection cycle (Figure 4. 15B). In the absence of IL-25 there were no differences in the worm burdens in the lung following challenge infection when compared to the wild type control (Figure 4. 15B). As expected there were less worms seen in the gut in the secondary response, however, delayed expulsion of the worms could again be seen in the IL-25 deficient mice (Figure 4. 15B). These data illustrate there is no significant impairment of protective responses against $N$. brasiliensis in IL-25 deficient animals during the lung 
phase of infection, however there is a minor role for efficient expulsion of L5 stage larvae from the small intestines.

A

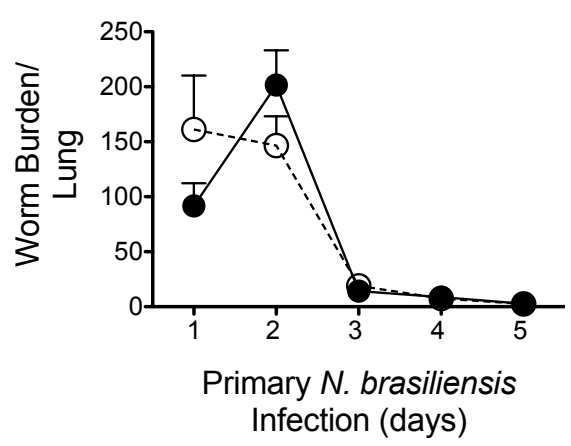

B

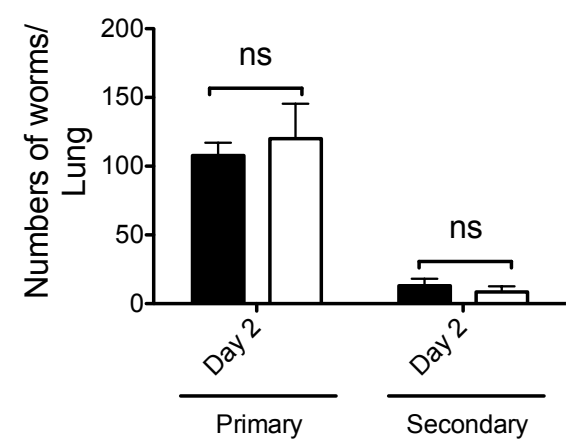

N. brasiliensis infection
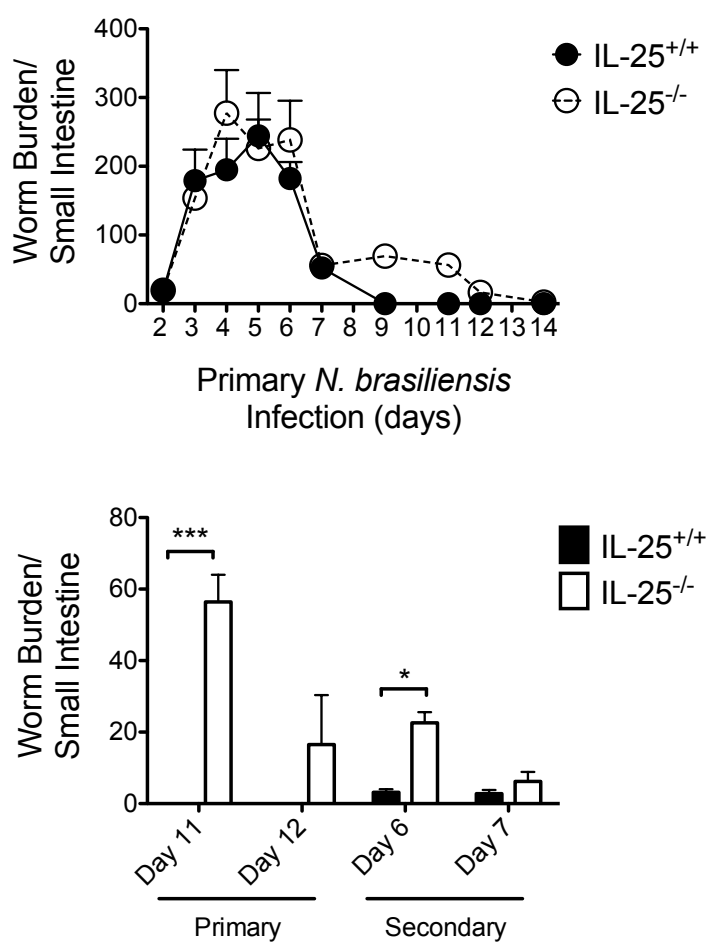

N. brasiliensis infection

Figure 4. 15 Lung worm burdens are unaffected by loss of IL-25 signalling however a minor delay in expulsion from the small intestine is noted following both primary and secondary infections. Viable worms were counted following migration out of harvested and processed lungs or small intestines following $\underline{\mathbf{A}}$ : primary $N$. brasiliensis infection or $\underline{\mathbf{B}}$ : secondary $N$. brasiliensis infections at times indicated. ${ }^{*} p \leq 0.01,{ }^{*} p \leq 0.001,{ }^{*} * \mathrm{p} \leq 0.0001 ; 2$ Way ANOVA with a Bonferroni post test, Bar and line graphs show mean \pm SEM and are representative of 2 or more experiments, $\mathrm{n} \geq 5$ mice/group. ns: not significant.

The Th2 associated cytokines IL-4, IL-5, IL-9 and IL-13 work co-operatively towards expulsion of $N$. brasiliensis from the small intestine ${ }^{167}$. IL-4 compensates for the absence of IL-5 and IL-9 but cannot compensate for the loss of IL-13 during a primary infection ${ }^{167,168}$. However, IL-4 has been determined as a critical factor for maintaining immunity and low worm burdens during the lung phase of $N$. brasiliensis secondary infections ${ }^{161}$. This section set out to investigate whether the loss of signalling of both IL-4 and IL-25 would result in loss in protection in either the lung or the gut phases of 
Chapter Four: The role of IL-25 in the generation of protective T helper type 2 responses against infection by parasitic helminths.

$N$. brasiliensis secondary infections. IL-25 deficient animals on IL-4 sufficient, IL-4 heterozygous and IL-4 deficient backgrounds were infected with $N$. brasiliensis alongside IL-25 sufficient controls with matching IL-4 backgrounds. To determine viable worm burdens, lungs were harvested 2 days after infection while small intestines were harvested 6 and 9 days after infection. For secondary worm burdens a challenge infection was administered 45 days following the primary infection and worm burdens were determined similarly following 2, 6 and 12 days. Dual cytokine deficient IL-4 ${ }^{-/}$IL-25 $5^{-/}$animals had similar worm burdens in the lungs to those detectable during the primary infections (Figure 4. 16A). In the lungs there was no difference between the worm burdens enumerated from the IL- $25^{-/}$animals as compared to the respective control mice. The loss of an allele of IL-4 had significant implications in the lungs on the worm burdens but no effect was detectable in the small intestines following secondary infection in comparison to the wild type $\mathrm{IL}_{-} 4^{+/+}$ worm burdens (Figure 4. 16B). Moreover, worm burdens in the small intestine 6 days following secondary infection was significantly higher in the IL-4 deficient and with a trend for higher numbers in the dual IL-4 and IL-25 deficient animals (Figure 4. 16B). This suggests a role for IL-4 in protection against re-infection from $N$. brasiliensis generated in the lungs that results in more worms making it through to the small intestine and therefore higher worm burdens are noted in this organ. Little role was noted for IL-25 in expulsion of worms from the small intestine following both primary and secondary infections, even though a clear role has been previously documented for expulsion following primary infection ${ }^{61}$. 
A

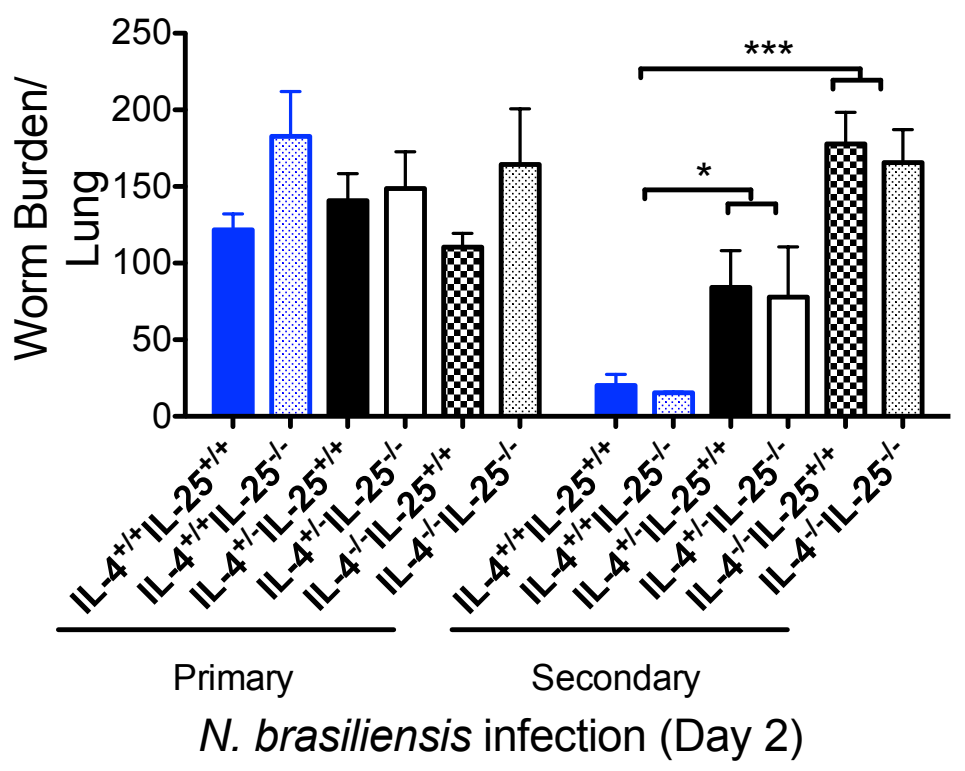

B

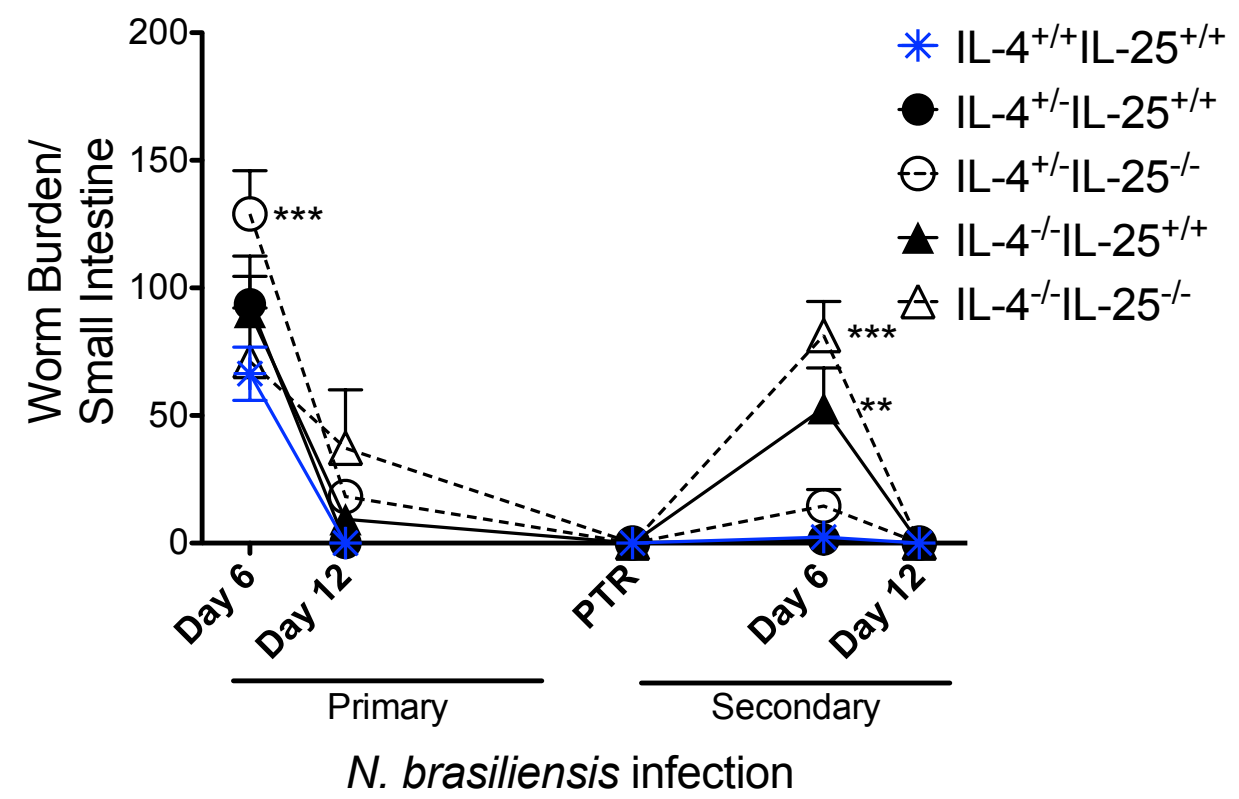

Figure 4. 16 IL-4 and IL-25 dual cytokine deficient animals have significantly impaired protective responses against secondary infections with $\boldsymbol{N}$. brasiliensis. Viable worms were counted following migration out of harvested and processed A: lungs or B: small intestines following primary and secondary $N$. brasiliensis infections at times indicated. ${ }^{*} p \leq 0.01$, $* * p \leq 0.001, * * * p \leq 0.0001 ; 2$ Way ANOVA with a Bonferroni post test as compared to IL-4/IL-4, Bar graphs show mean \pm SEM and are representative of 2 or more experiments, $n \geq 6$ mice/group. PTR: Prior to re-infection. 
Chapter Four: The role of IL-25 in the generation of protective T helper type 2 responses against infection by parasitic helminths.

\subsection{Th2 cells, type 2 cytokines and nuocytes in the mesenteric lymph node show no deficiency in the absence of IL-25}

The delay in expulsion of worms from the intestine is thought to arise due to a delay in the type 2 responses in the local tissue or the draining lymph node. The lack of expansion of IL-13 producing nuocytes in the absence of IL-25 is theorised as the cause for this delayed expulsion ${ }^{61,175}$. To determine whether the Th2 adaptive immunity was similar in the small intestine in the absence of IL-25 following infection with $N$. brasiliensis, the proportion and numbers of mesenteric lymph node Th2 cells at day 9 following primary infection were investigated. While the frequency of Th2 cells in the absence of IL-25 was significantly lower in comparison to the IL25 sufficient control, no differences were evident in the total numbers of Th2 cells in both the presence and absence of IL-25 regardless of the ability to signal through IL-4 (Figure 4. 17). A multiplex cytokine assay was also used to determine the type 2 cytokine responses 9 days following either primary or secondary infection with $N$. brasiliensis. Mesenteric lymph nodes were re-stimulated with $\alpha \mathrm{CD} 3 / \alpha \mathrm{CD} 28$ beads in the presence of rIL-2 for 24 hours and the amount of IL-4, IL-13, IL-5 or IL-3 was determined in the supernatant. No deficiencies were noted in any of the cytokines tested, in fact a trend towards higher concentrations of IL-4, IL-5 and IL-3 were detected in the absence of IL-25 signalling with IL-13 concentrations of a significantly higher concentration during the primary response (Figure 4. 18). Remarkably a time course following the expansion of mesenteric lymph node nuocytes after primary infection of $N$. brasiliensis determined no significant differences in frequencies or numbers of the innate effector cells (Figure 4. 19). Of note however is the possibility that the delay in nuocyte induction occurred earlier than day 6. This would be supported by data from IL-17BR ${ }^{-/}$(IL-25 receptor deficient) animals where a deficiency in nuocyte numbers is noted on day 4 but by day 11 post primary infection markedly higher numbers of nuocytes are already detectable as compared to the IL-17BR ${ }^{+/+}$controls ${ }^{61}$. In summary these data illustrate that in the absence of IL-25 signalling there are no detectable defects in Th2 cell numbers, Th2 associated cytokines or nuocytes in the mesenteric lymph nodes following infection with N. brasiliensis. 


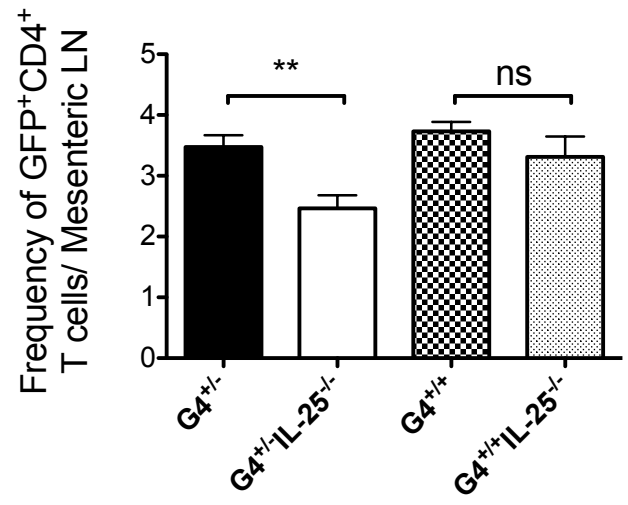

N. brasiliensis infection

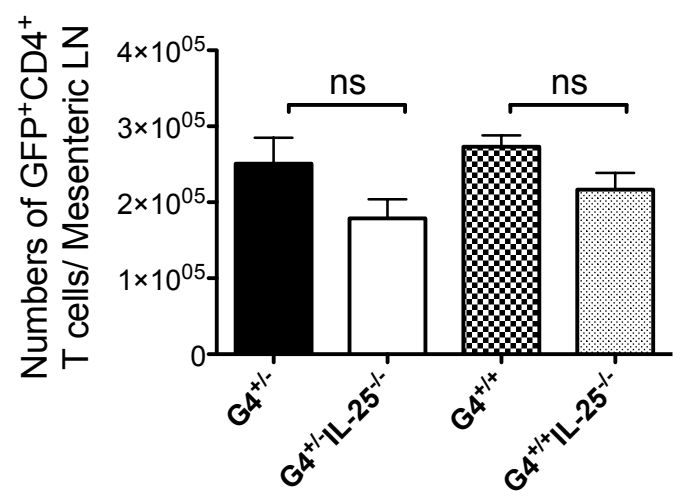

N. brasiliensis infection

Figure 4. 17 Mesenteric Th2 cells differentiate in the absence of IL-25 following infection with $\mathbf{N}$. brasiliensis. Mesenteric lymph nodes (MesLN) were harvested 9 days following primary infection with 600 L3 SQ N. brasiliensis and processed into single cell suspensions, stained and analysed by flow for frequency of Th2 cells (Doublet DAPICD $3^{+} \mathrm{CD} 4^{+} \mathrm{GFP}^{+}$cells) that were enumerated as total numbers of Th2 cells per lymph node. ${ }^{*} p \leq 0.01,{ }^{* *} p \leq 0.001$, $*^{* *} \mathrm{p} \leq 0.0001 ; 1$ Way ANOVA with a Tukey post test. Bar graphs show mean \pm SEM and are representative of 2 or more experiments, $n \geq 5$ mice/group. ns: not significant. 
Chapter Four: The role of IL-25 in the generation of protective T helper type 2 responses against infection by parasitic helminths.

IL-4

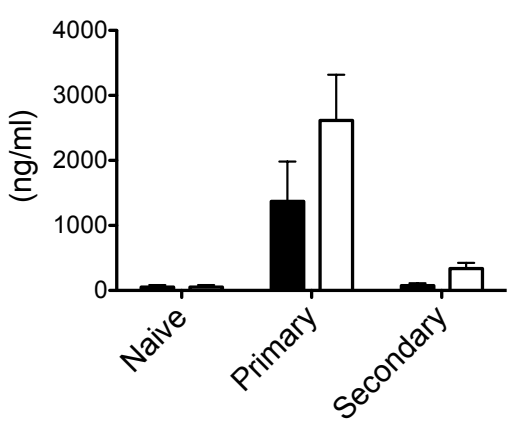

IL-5

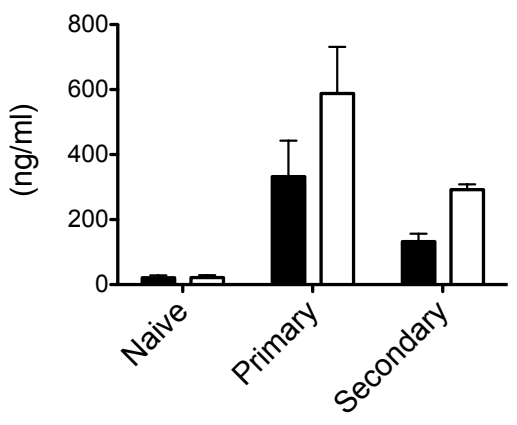

IL-13

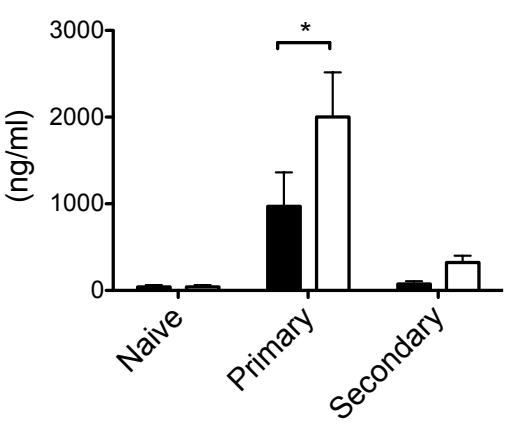

IL-3

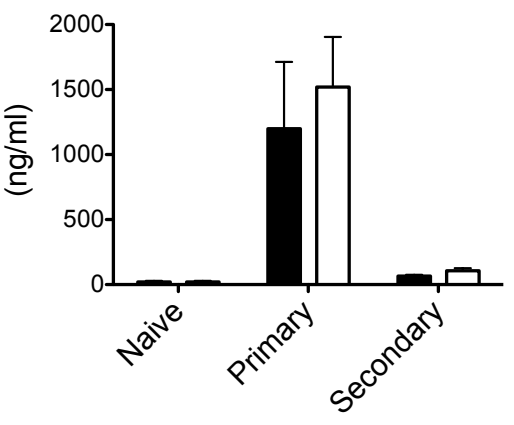

Figure 4. 18 Type 2 cytokines from the mesenteric lymph nodes of IL-25 deficient mice were similar to those of the IL-25 ${ }^{+/+}$controls following $\mathbf{N}$. brasiliensis infection. Mesenteric lymph nodes from day 9 primary and day 9 secondary $N$. brasiliensis infected $\mathrm{IL}-25^{+/+}$and $\mathrm{IL}-25^{-/}$ mice were processed into single cell suspensions and re-stimulated on $\alpha \mathrm{CD} 3 / \alpha \mathrm{CD} 28$ beads for $24 \mathrm{hrs}$ in presence of $100 \mathrm{U} / \mathrm{ml} \mathrm{IL-2}$. Supernatant was analysed using bioplex analysis for IL4, IL-5, IL-13 and IL-3. ${ }^{*} p \leq 0.01,{ }^{* *} p \leq 0.001,{ }^{* * *} p \leq 0.0001 ; 2$ Way ANOVA with a Bonferroni post test; Bar graphs show mean $\pm \mathrm{SEM}, \mathrm{n} \geq 3$ mice/group. 


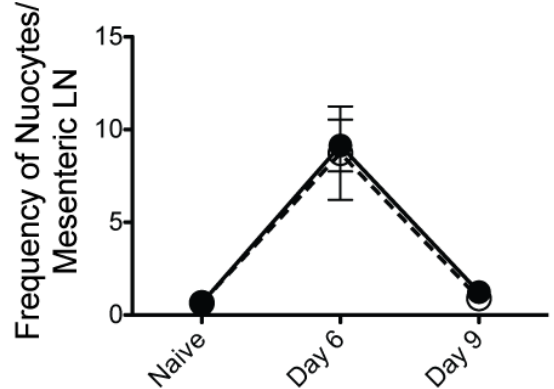

N. brasiliensis infection

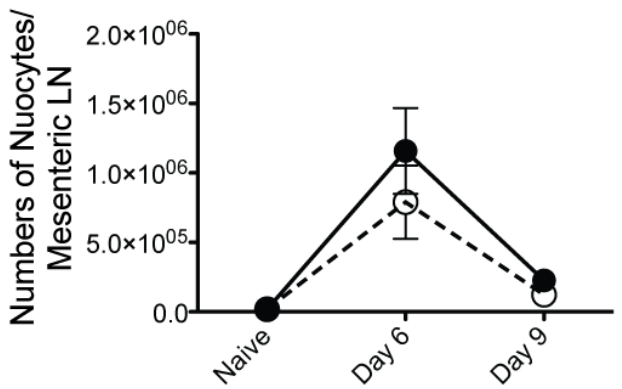

N. brasiliensis infection
- IL-25+/+

$\theta \mathrm{IL}-25^{-1-}$

Figure 4. 19 Nuocyte frequency and numbers in the mesenteric lymph nodes of IL-25 deficient mice were similar to those of the controls following primary $\mathbf{N}$. brasiliensis infection. Mesenteric lymph nodes from naïve, day 6 and day 9 primary $N$. brasiliensis infected IL-25 $5^{+/+}$and IL-25 $5^{-/-}$mice were processed into single cell suspensions, stained and analysed by flow cytometry for frequency of Nuocytes (doublet ${ }^{-} \mathrm{DAPI}^{-} \mathrm{SSC}^{\mathrm{lo}}$ Lineage $^{-} \mathrm{Sca1}^{+} \mathrm{IL}_{-}$ $7 \mathrm{R}^{+} \mathrm{ICOS}^{+}$) that were enumerated as total nuocytes per lymph node. ${ }^{*} \mathrm{p} \leq 0.01,{ }^{* *} \mathrm{p} \leq 0.001$, $* * * p \leq 0.0001 ; 2$ Way ANOVA with a Bonferroni post test. Line graphs show mean $\pm S E M, n \geq 4$ mice/group.

\subsection{H. polygyrus expulsion from the gut is delayed in IL-25 deficient animals}

Similar to immunity against $N$. brasiliensis, the cytokines IL-4, IL-13 along with CD4 $\mathrm{T}$ cells play an integral part in generating protective immunity against challenge infections with $H$. polygyrus ${ }^{153,217}$. The primary infection is a chronic infection, however treatment with anti-helminthic drugs inducing parasite expulsion results in immunity to challenge infections in the animals. H. polygyrus is an entirely enteric parasite where the initial 8-10 days are spent burrowed into the submucosa of the small intestine. During challenge infections the immune system is seen to form a granulomatous mass with associated limits in worm numbers, size and fecundity noted upon exit into the lumen ${ }^{183}$. Once adult worms have exited the submucosa and entered the lumen the 'weep and sweep' mechanisms similar to those reported during $N$. brasiliensis infections are likely to occur enabling the removal of adult L5 stage worms ${ }^{29}$.

To determine whether delayed expulsion following helminth infection in IL-25 deficient animals also occurred with the gut dwelling nematode $H$. polygyrus, IL- $25^{-/-}$ 
Chapter Four: The role of IL-25 in the generation of protective T helper type 2

responses against infection by parasitic helminths.

and $\mathrm{IL}-25^{+/+}$control animals were infected intragastrically (i.g.) with $200 \mathrm{~L} 3 \mathrm{H}$. polygyrus, treated with anti-helminthic drug and re-infected 36 days later. Viable worms were recovered from the small intestines 10 and 20 days following primary and secondary infections. At these same time points, total numbers of eggs per caecum were determined using a modified McMaster's technique. The McMaster's technique makes use of a known mass of faeces resuspended in a known volume of water, counted in a McMaster counting chamber of which a known volume is counted, therefore the numbers of eggs can be calculated per gram of faeces. Delayed expulsion was noted following secondary infection but only after 20 days (Figure 4. 20). At day 10 following secondary infection, shortly after the worms have emerged into the lumen, no differences were observed between IL- $25^{-/-}$or control animals. Apart from 10 days after primary infection with $H$. polygyrus, no significant differences were observed in the egg counts or fecundity of worms recovered from the intestines in the absence of IL-25 signalling. These data illustrate a role for IL-25 in the expulsion of the entirely enteric helminth $H$. polygyrus. Further experiments would be needed to determine whether nuocytes could possibly be responsible for this altered phenotype.
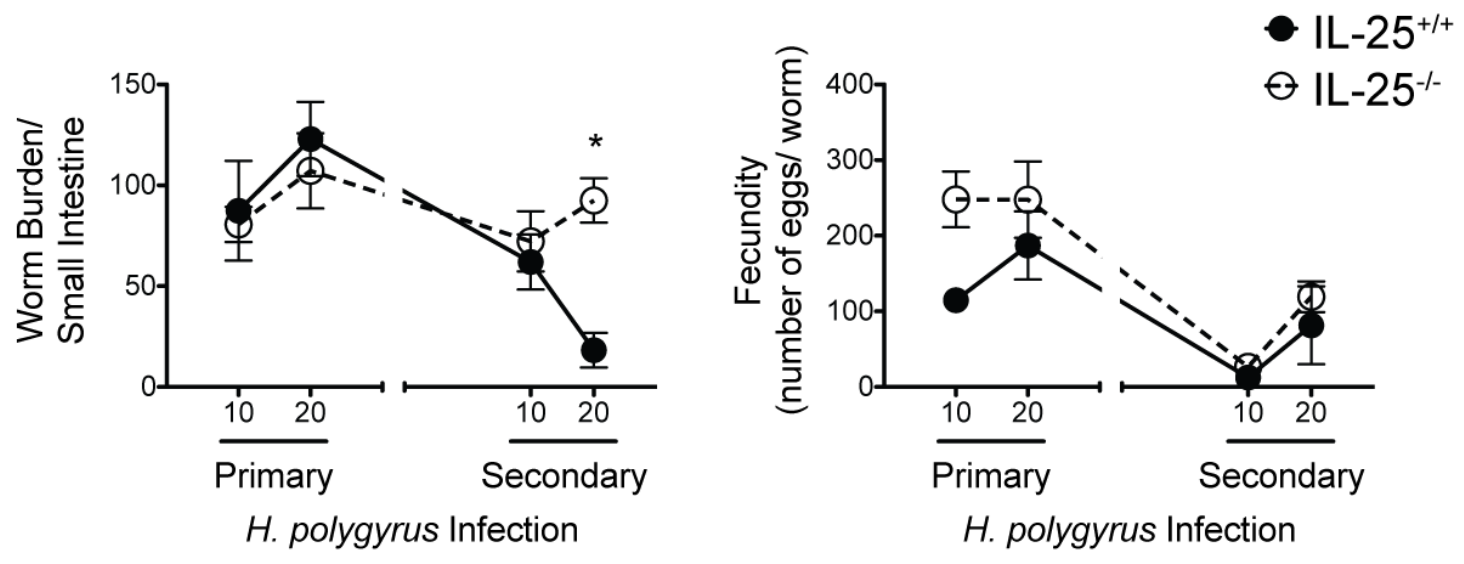

Figure 4. 20 Loss of IL-25 resulted in delayed expulsion from the gastrointestinal tract following secondary infection with $\boldsymbol{H}$. polygyrus. IL- $25^{+/+}$controls and $\mathrm{IL}-25^{-/-}$mice were infected i.g. with 600 L3 H. polygyrus, treated with anti-helminthic and re-infected 36 days later. Viable worm burdens, egg counts and fecundity of worms were determined 10 and 20 days following both primary and secondary infections. Viable worms were counted following migration out of harvested and processed small intestines, total eggs per caecum were counted using the modified McMaster technique and fecundity was determined as number 
Chapter Four: The role of IL-25 in the generation of protective T helper type 2 responses against infection by parasitic helminths.

of eggs per worm. ${ }^{*} p \leq 0.01,{ }^{* *} p \leq 0.001,{ }^{* * *} p \leq 0.0001 ; 2$ Way ANOVA with a Bonferroni post test as compared to $\mathrm{IL}-25^{+/+}$. Line graphs show mean $\pm \mathrm{SEM}, \mathrm{n} \geq 5$ mice/group. 
Chapter Four: The role of IL-25 in the generation of protective T helper type 2 responses against infection by parasitic helminths.

\subsection{Discussion}

It was hypothesised that the absence of IL-25 would result in delayed and quite possibly decreased $\mathrm{T}$ helper type 2 responses. Surprisingly this was not the outcome of the research contained within this chapter. There were no delays or deficiencies between the IL-25 deficient animals and their wild type controls in either the innate or adaptive Th2 responses measured. Neither was there any role determined for IL-25 in the generation of protective immunity against re-infection. The protective response was mediated by a fully developed Th2 immune response where total numbers of Th2 cells in the lymph nodes and lungs resembled that of IL-25 sufficient controls. The increased blood basophilia, eosinophilia, serum IgE titres and trend towards increased mucous secretion and all infer a role for IL-25 in regulation of over production of type 2 responses associated with helminth infections rather than induction of these responses. The only apparent roles for IL-25 inducing Th2 immunity were that of timely expulsion of both primary and to a lesser degree secondary infections of $N$. brasiliensis from the small intestines. Additionally a role for IL-25 during intestinal helminth expulsion was noted in $H$. polygyrus secondary infections. In summary, this chapter showed no role for IL-25 in initiating or maintaining a Th2 immune response, with the only detectable role in mediating timely expulsion of helminths from the intestine.

Unlike previous allergic airways inflammatory (AAI) models where IL-25 was required during sensitization and challenge for the development of the Th2 responses and associated pathologies ${ }^{218,219}$, this chapter reported no role for IL-25 in mediating Th2 driven inflammatory responses in the lung that resulted from infection with $N$. brasiliensis. The airways inflammatory response to $N$. brasiliensis resembles allergic airway models with features such as lung eosinophilia, airways hyperreactivity and mucous production ${ }^{159,160,220}$. In the absence of IL-25 similar numbers of both adaptive and innate AHR responses, the Th2 cells and eosinophilia, were detectable to similar degrees in the lungs. Blood basophilia, eosinophilia and serum $\operatorname{IgE}$ titres were increased and mucous production in the bronchioles was also slightly increased. The reduced Th2 responses described previously when IL-25 signalling was ablated were associated with an altered immune phenotype, with decreased IL-5 and IL-13 
production and increased IL-17 production. This switching of immune phenotypes in the absence of IL- 25 from the Th2 dominated arm to that of Th17 or Th1 dominated arm was reported in response to $T$. muris infection ${ }^{145}$. During autoimmune encephalomyelitis (EAE) IL-25 was also shown to promote the Th2 arm to downregulate the autoimmune Th17 arm ${ }^{194}$. Unlike these previous reports, this chapter found no changes in IL-4, IL-5, IL-13, IL-9 or IL-17 during the lung allergic inflammatory responses. A possible explanation for the differences noted for the role of IL-25 in other models of AAI, T. muris infections and EAE could be explained by the relatively weak immune Th2 phenotypes induced by T. muris infections and OVA models of AAI, unlike the robust Th2 induction and all round activation associated with an $N$. brasilienis or $H$. polygyrus infection. The comparison between weak and robust Th2 immune phenotypes can be noted due to the markedly higher levels of serum IgE, basophilia, eosinophilia and mucous producing goblet cells in an $N$. brasilienis and $H$. polygyrus infection in comparison to OVA models of AAI and infection with T. muris. Another explanation could be the reliance on IL-13 for mediating AAI, T. muris infections and EAE whereas $N$. brasiliensis and H.polygyrus protective immunity relies upon the production of IL-4. Indeed, IL-4 was confirmed in this chapter as predominantly produced by Th2 cells during secondary infections in the lung. While previous studies infer IL-25 may have a role in negative regulation of the Th1/Th17 arms of the immune response the studies in this chapter did not support this theory, with similar levels of Th1 and Th17 cytokines in both the IL-25 deficient and sufficient animals.

Of interest is the delayed intestinal expulsion observed during both $N$. brasiliensis and $H$. polygyrus infection. $H$. polygyrus challenge infections require CD4 T cells during larval development stages until day 8 after challenge then a CD4 T cell independent luminal resistance occurs day 8 onwards ${ }^{183}$, timing that coincides with the delayed expulsion seen in Figure 4. 20. A possible explanation for the delayed expulsion of $H$. polygyrus is, the ablation of IL-25 signalling through parenchymal cells such as the smooth muscle cells resulting in a defect in the 'weep and sweep' mechanisms of the intestine. IL-25R is present on smooth muscle cells ${ }^{221}$ and IL-25 is produced by Th2 cells ${ }^{138}$, mast cells ${ }^{143}$, basophils ${ }^{147}$ and eosinophils ${ }^{144}$; all effector cells readily found in the small intestines of $N$. brasilienisis infected animals. Although Ballantyne et al. 
Chapter Four: The role of IL-25 in the generation of protective T helper type 2 responses against infection by parasitic helminths.

were unable to demonstrate a direct effect of IL-25 on airways smooth muscle cells ${ }^{219}$, in a paper published subsequently the validity of the rIL-25 used in those experiments was questioned ${ }^{218}$. Another explanation for the delayed expulsion could be as a result of a deficiency in IL-13 producing nuocytes as has previously been proposed ${ }^{61}$, however this chapter determined no significant defect in nuocyte numbers in the mesenteric lymph nodes. There is the possibility that this chapter determined no differences in numbers of nuocytes as the time course evaluated began too late. The delay in nuocyte expansion in the intestine has been previously reported as early as 4 days post infection ${ }^{61}$, this chapter investigates nuocytes numbers at 6 and 9 days after infection. However what is striking to note is the lack of correlation between early deficiency in nuocyte numbers that occurs prior to 6 days after infection and increased worm burdens that are noted from day 7 after infection. Although it is possible with the lack of an IL-13 reporter the IL-13 positive nuocytes were significantly ablated and thereby unable to effect expulsion of the parasite. Another possibility may include the necessity for lineage negative cells to become activated by IL-25 to mediate their effects in the small intestinal environment ${ }^{150,211}$. Thus, whilst there were no differences in nuocyte numbers detectable in this chapter, there may be a marked difference in effectiveness of these enumerated cells. In vivo data substantiating this theory illustrates that both innate helper 2 (Ih2) cells ${ }^{150}$ and nuocytes ${ }^{211}$ require rIL-25 to induce effector responses following adoptive transfer of these innate cells, inferring IL-25 is required to activate nuocytes to enable them to perform their effector functions. This theory also correlates with previously published IL-25 mRNA levels detectable in the small intestine following primary infection with N. brasiliensis. IL25 mRNA levels are shown to increase only after 7 days of primary infection ${ }^{136}$, coinciding with the delayed expulsion in IL-25 deficient mice that is quantifiable from 7-12 days. These studies suggest that nuocytes require activation by IL-25 for subsequent expulsion of parasites from the intestine. As such this theory would also offer a possible explanation for the increased worm burdens noted later during $H$. polygyrus infection.

In conclusion, these data demonstrate no role for IL-25 in the induction and maintenance of adaptive $\mathrm{T}$ helper type 2 responses to infection with $N$. brasiliensis. However this research does demonstrate distinct differences between Th2/type-2 
Chapter Four: The role of IL-25 in the generation of protective T helper type 2 responses against infection by parasitic helminths.

effector responses in the lung and the intestines and highlights the importance of using a variety of Th2 models to determine the functional effects of novel cytokines. 



Chapter Five:

Characterisation of a Th2 cell: The allelic expression of IL-4. 


\subsection{Background}

Functionally distinct T helper subsets were originally defined in the 1980's when the Th1 and Th2 subsets were described on the basis of the production of their canonical cytokines, IFN $\gamma$ or IL-4 ${ }^{6}$. Visualising intracellular production of IL-4 is technically difficult due to the short half-life of the protein. Less invasive methods of distinguishing Th2 cells based on surface molecule expression would be beneficial for Th2 cell isolation and analysis. A variety of surface molecules have been investigated including: the IL-2 receptor (CD25) ${ }^{222}$ that was shown to be expressed early during the development of Th2 cells, the IL-4 receptor (CD124) that was shown to be upregulated early after antigen experience but downregulated in differentiated Th2 cells ${ }^{223}$ and the chemokine receptors CCR3 (CD193) ${ }^{224}$, CCR4 (CD194) ${ }^{225,226}$, CCR8 (CD198) ${ }^{227}$, CRTh2 ${ }^{228}$ and the innate cytokine receptors, IL-33 receptor (T1/ST2) 229,230 and IL-25 receptor (IL-17RB) ${ }^{81,147}$ all of which vary in their levels of expression within the Th2 cell population. Therefore researchers rely on the intracellular detection of IL-4 for confirmation that the CD4 T cell can be classified as a Th2 cell.

The production of IL-4 by Th2 cells is closely regulated as IL-4 signalling orchestrates both protective responses against parasitic helminth infections ${ }^{182,217}$ and aberrant inflammatory responses to allergens ${ }^{231-233}$. There are multiple methods of regulating the production of IL-4, however the interest of this chapter is how IL-4 expression is regulated on an allelic basis. Diploid genomes were theorised as a safety mechanism whereby biallelic expression ensured any deleterious mutation on a single allele would still result in the functional expression of the gene product from the opposite allele. More recently however, it has been demonstrated that not all alleles are expressed in pairs, that in fact, certain genes express one allele independently from the other; this form of gene regulation is termed monoallelic expression. Monoallelic expression was initially considered a rare event however breakthroughs in molecular technology have led to speculation that monoallelic expression may be far more common than initially thought. This has led to the suggestion that monoallelic expression may be providing the cell with a chance for diversity which would be unobtainable if both alleles are always expressed simultaneously ${ }^{234}$. 
The discovery of predominantly monoallelic expression of IL-4 by in vitro generated Th2 cells and the simultaneously reported low frequency of total $\mathrm{T}$ helper cells that actually become IL-4 producers ${ }^{119-121}$, lead to speculation that either there is an inefficient process of inducing accessibility at the IL4 gene ${ }^{235,236}$ or there is some stochastic process that determines which cells produce IL-4 and which cells don't ${ }^{119}$. The idea being that the stochastic opening of an allele is an infrequent occurrence. The 'on/off' or probabilistic manner in which IL-4 alleles were shown to be expressed resulted in a further intriguing theory as to how this may serve as a regulatory mechanism during a Th2 immune response ${ }^{237}$. To elaborate, IL-4 is thought to be secreted in a polarised fashion through a synapse to the cell of interest (e.g. a Th2 cell helping a B cell), therefore considering only a limited number of $\mathrm{T}$ helper cells are producing IL-4 they will only be able to interact with a limited number of B cells and therefore limit the downstream effects of IL-4 signalling. This creates a stringent form of regulation as the greater the number of Th2 cells the greater the effector responses. If however IL-4 acts not only in the local vicinity but also has far reaching effects, then the overall quantity of IL-4 released by the total Th2 cell population would be of vital importance for the effector response and tailoring the quantity released by $\mathrm{Th} 2$ cells rather than tailoring the quantity of Th2 cells would be a far more tricky system to regulate. Interestingly this theory of cell to cell mediated signalling by IL-4 does raise a query as to what happens when both alleles of IL4 are expressed simultaneously, do these helper T cells have twice the concentration of IL-4 to secrete in a polarised fashion at the cell synapse and therefore twice the downstream effects?

The total Th2 cell subset is not a homogeneous population but comprises instead of further subsets of specialised Th2 cells with particular roles including for example: effector Th2 cells that mediate immunity against parasitic helminths ${ }^{153-155}$, central memory and effector memory Th2 cells that are located respectively in the lymph nodes or tissues and produce a rapid recall response upon antigen challenge ${ }^{161,183}$, and possibly the $\mathrm{T}$ follicular helper (Tfh) cells or a subset of CD4 $\mathrm{T}$ cells that are responsible for class switch recombination to IgE through the production of IL-4 ${ }^{109-}$ ${ }^{111}$. The similarity between these subsets of CD4 Th2 cells is their production of IL-4. Since il4 allele expression by CD4 $\mathrm{T}$ cells is highly regulated in vitro, this chapter queried whether the Il4 gene is subject to monoallelic or biallelic expression in vivo. 
Chapter Five: Characterisation of a Th2 cell: The allelic expression of IL-4.

IL-4 producing CD4 $\mathrm{T}$ cells in vivo have recently been shown to utilise different transcription factors in lymphoid compared to tissue environments ${ }^{111,117,118}$, therefore it was also decided to determine whether the genomic organisation in terms of allelic expression of IL-4 was also subject to specific regulation within these various specialised Th2 cells. 
Chapter Five: Characterisation of a Th2 cell: The allelic expression of IL-4.

\subsection{Research Objectives}

To determine whether the genomic organisation, which reflects a commitment to Th2 cytokine expression, could provide a clearer definition of an in vivo functional Th2 cell.

Summarised aims:

- Investigate whether T helper cell stage of development, tissue location or type of initiating stimulus is associated with particular allelic expression of IL-4 by Th2 cells.

- Determine whether the presence of one or both alleles can be linked to a functionally relevant mechanism involved in the generation of protective immunity against parasitic helminth infections. 


\subsection{Results}

\subsection{Monoallelic expression of IL-4 is the predominant form of regulation in both effector and memory Th2 cells}

Previous investigations into the allelic expression of IL-4 have used methods of in vitro Th2 differentiation. This chapter set out to examine the regulation of allelic expression of IL-4 after in vivo Th2 differentiation. Homozygous KN2/KN2 ${ }^{190}$ and G4/G4 ${ }^{119}$ IL-4 reporter mouse strains were crossed, to generate an il4 allele specific reporter mouse. The KN2 reporter mice express modified human CD2 (hCD2) while the G4 reporter mice express green fluorescent protein (GFP) instead of IL-4 protein. This combination generated a novel tool, the KN2/G4 mouse, which enabled direct $e x$ vivo monitoring of the expression of independent alleles of the Il4 gene through assessment of the protein product.

A Th2 allergen model involving a activation of a single draining lymph node through various known Th2 allergens was used to determine whether Th2 cells generated in vivo were subject predominantly to monoallelic expression, similar to in vitro generated Th2 cells. It was also of interest to further determine whether this expression pattern differed depending on the effector or memory status of the Th2 cell, a Th2 allergen model was used. The allele specific reporter mice (KN2/G4 mice) were injected with the common allergen, house dust mite (HDM), in the dermis of the ear that results in the differentiation and expansion of Th2 cells reaching maximum numbers within 7 days of the injection. Thirty days later once the immune response had subsided, these animals were challenged with the same allergen dose to induce a rapid memory response. The draining lymph nodes were harvested, processed into single cell suspensions and counted using trypan blue exclusion dye to determine total viable cells (Figure 5. 1A). A memory response was detectable upon secondary challenge with the same antigen; the total number of cells recruited to the lymph node within the three-day period following challenge was higher after secondary challenge in comparison to the primary immune response. The total numbers of lymphoid cells dropped markedly following primary infection but were still significantly higher than 
naïve numbers at day 30 following primary infection. To confirm the Th2 immune response had subsided at 30 days after the injection, the CD4 T cells were monitored for the expression of GFP protein, indicative of Th2 cell commitment to IL-4 production. The total frequencies of Th2 cells were at least 3 times lower at day 30, the day of HDM challenge, compared to frequencies of Th2 cells during the height of the primary response or secondary response (Figure 5. 1A). This model system was used to determine whether the expression of in vivo generated Th2 cell IL-4 production was monoallelic or biallelic and whether there was a tendency towards either monoallelic or biallelic expression during the primary or secondary immune response.

To monitor expression of IL-4, the lymph node cells were harvested, processed, stained and analysed by flow cytometry and the Th2 cells were monitored for the expression of reporter proteins (Figure 5.1B). The expression of either monoallelic GFP or hCD2 or biallelic GFP and hCD2 together were graphically represented as frequency of reporter protein expression per CD4 T cells (Figure 5. 1C), this analysis illustrated that in the majority of Th2 cells monoallelic expression of IL-4 was significantly higher than biallelic expression of IL-4 during both the primary and the secondary immune response. The only exception was in the small memory Th2 cell population visible prior to challenge at day 30 where the differences between the frequencies of CD4 $\mathrm{T}$ cells with monoallelic or biallelic expression of IL-4 is markedly less striking. In summary, as had been seen previously in vitro, the majority of Th2 cells in vivo regulated IL-4 through monoallelic expression with little variation during primary and secondary lymphoid immune responses. 
A
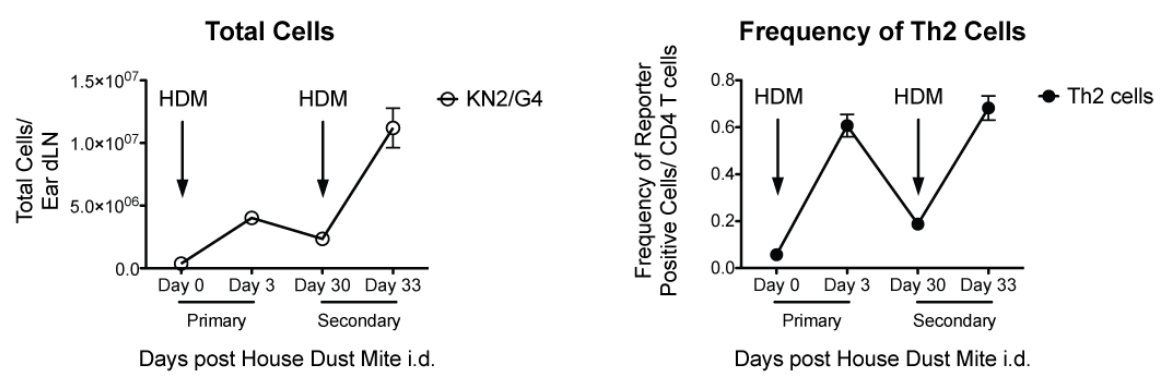

B

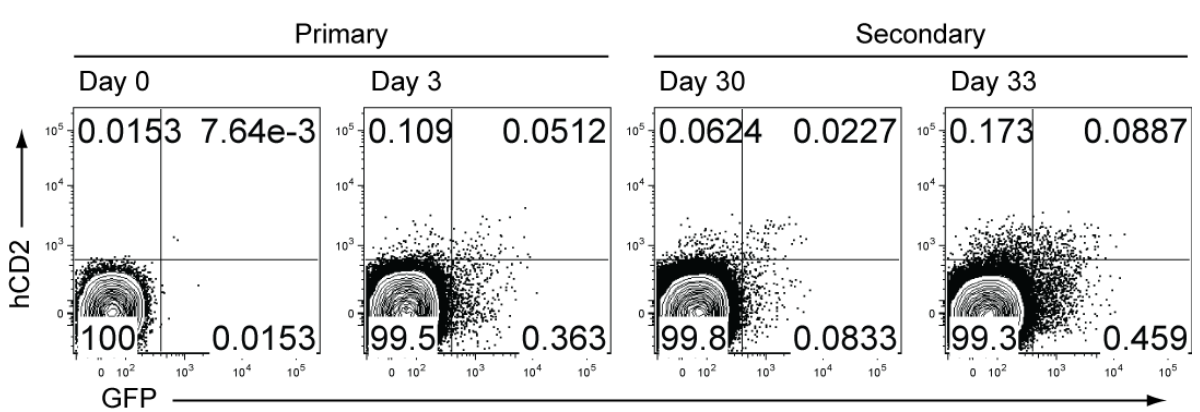

C

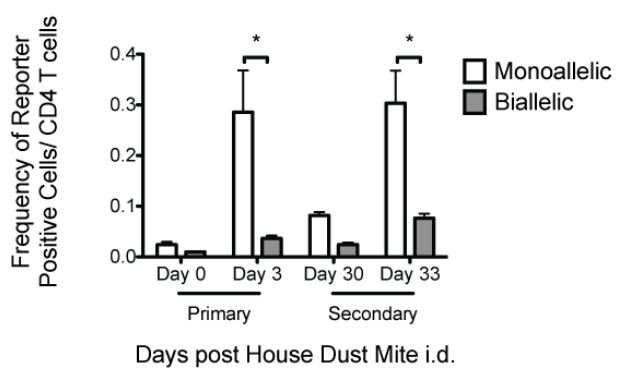

D
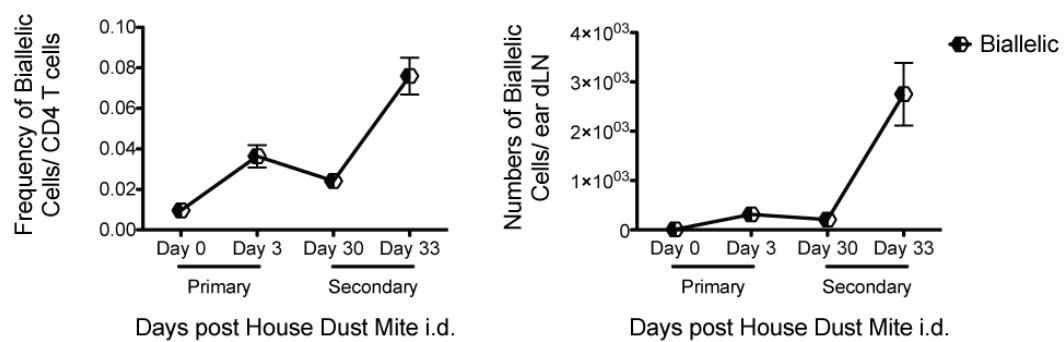

Figure 5. 1 IL-4 expression is predominantly monoallelic, however the biallelic IL-4 population increases as the immune response increases. House dust mite was injected into the ear dermis with a challenge injection administered at 30 days after the primary injection. The draining lymph node immune response was tracked over time monitoring $\underline{\mathbf{A}}$ : the total numbers of cells present in the lymph node as determined by viable exclusion dye and the frequency of CD4 T cells expressing IL-4 reporter proteins was represented as a line graph. B: the expression of hCD2 or GFP by the CD4 T cells determined by flow cytometry. Contour plots representative of day $0,3,30$ and 33 and inset numbers representing frequency of reporter positive cells per CD4 T cells. $\underline{\mathrm{C}}$ : The frequency of monoallelic or biallelic reporter positive cells per CD4 T cells. $\underline{\mathbf{D}}$ : The frequency of biallelic cells per CD4 T cells and total biallelic cells per lymph node plotted over time. ${ }^{*} p \leq 0.01,{ }^{* *} p \leq 0.001,{ }^{* *} p \leq 0.0001 ; 2$ Way ANOVA with a Bonferroni post test as compared to biallelic Th2 cells. Data is representative of at least 2 independent experiments with bar and line graphs showing mean \pm SEM for $n \geq 4$ mice per group. i.d.: intradermal 


\subsection{Th2 cells with biallelic IL-4 expression have a higher activation status than Th2 cells with monoallelic IL-4 expression}

An interesting correlation was observed between the increasing total Th2 immune response and both the frequency and total numbers of biallelic IL-4 expressing Th2 cells (Figure 5. 1 D). This observation of a possible link between the increasing Th2 immune response and the proportion of biallelic IL-4 expressing Th2 cells lead to a theory that Th2 cells with biallelic IL-4 expression may be functionally distinct from monoallelic IL-4 expressing Th2 cells. To investigate this theory further, the Th2 cells derived from allergen-induced primary and secondary responses (as described in Figure 5.1) were stained for cell surface markers discerning their activation status. The median fluorescent intensity of the early activation marker CD69 along with the activation marker CD44 were significantly increased in the biallelic cells compared with the monoallelic or reporter negative cells while the memory cell associated markers CD45RB and CD62L remained similar to that detected on the surface of the monoallelic cells (Figure 5. 2). Interestingly not only did Th2 cells with biallelic IL-4 expression have higher levels of activation markers, they also produced significantly more IL-4 reporter protein on a per cell basis (as measured by the median fluorescent intensity) compared to their monoallelic counterparts (Figure 5. 3). When restimulated and stained for the presence of additional cytokines a discrepancy arose between the GFP monoallelic cells and the hCD2 monoallelic cells with GFP monoallelic cells far more likely to express an additional cytokine, IL-13, IL-5, IL-10 or IFN $\gamma$ whilst expressing the IL-4 reporter protein in comparison to their hCD2 monoallelic counterparts (Figure 5. 4). In summary, the Th2 cells with biallelic IL-4 expression had a higher activation status and had increased per cell expression of reporter proteins than the Th2 cells with monoallelic IL-4 expression. 


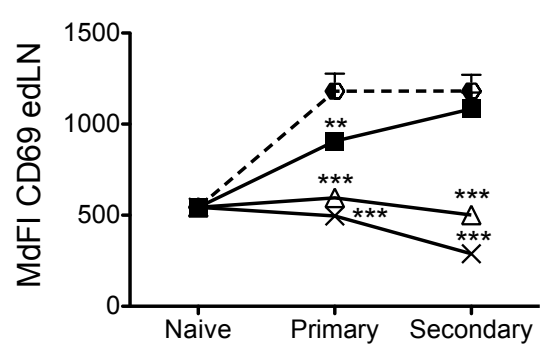

House Dust Mite i.d.

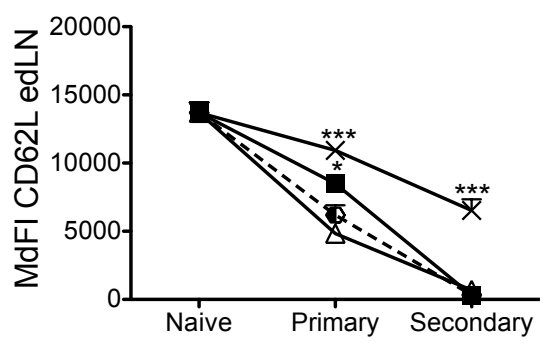

House Dust Mite i.d.

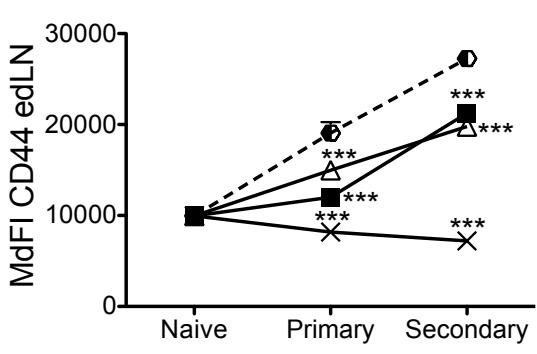

House Dust Mite i.d.

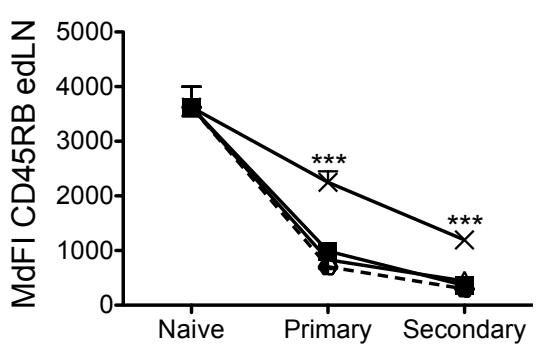

House Dust Mite i.d.

Figure 5. 2 Biallelic cells have a higher expression of activation markers compared to monoallelic cells. House dust mite was injected into the ear dermis with a challenge injection administered at 30 days; the draining lymph node immune response was tracked over time. The median fluorescent intensity of CD69, CD44, CD62L and CD45RB of CD4 T cells of hCD2 monoallelic or GFP monoallelic or reporter negative were compared to the biallelic (expressing both reporters) cells. ${ }^{*} \mathrm{p} \leq 0.01,{ }^{*} \mathrm{p} \leq 0.001,{ }^{* * *} \mathrm{p} \leq 0.0001 ; 2$ Way ANOVA with a Bonferroni post test as compared to biallelic cells. Data is representative of at least 2 independent experiments with line graphs showing mean $\pm S E M$ for $n \geq 4$ mice per group. i.d.: intradermal. edLN: ear draining lymph node.

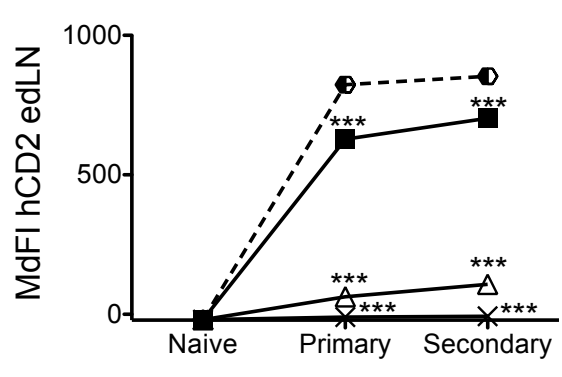

House Dust Mite i.d.

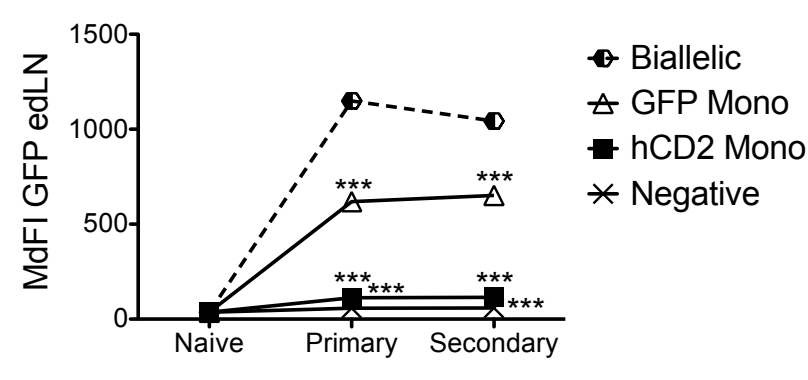

House Dust Mite i.d.

Figure 5. 3 Biallelic cells express more IL-4 reporter proteins per cell than monoallelic cells. House dust mite was injected into the ear dermis with a challenge injection administered at 30 days; the draining lymph node immune response was tracked over time. The median fluorescent intensity of hCD2 and GFP of CD4 T cells that were hCD2 monoallelic or GFP monoallelic were compared to the biallelic (expressing both reporters) cells. ${ }^{*} p \leq 0.01$, $* * p \leq 0.001, * * * p \leq 0.0001 ; 2$ Way ANOVA with a Bonferroni post test as compared to biallelic cells. Data is representative of at least 2 independent experiments with line graphs showing mean \pm SEM for $n \geq 4$ mice per group. id.: intradermal. edLN: ear draining lymph node. 

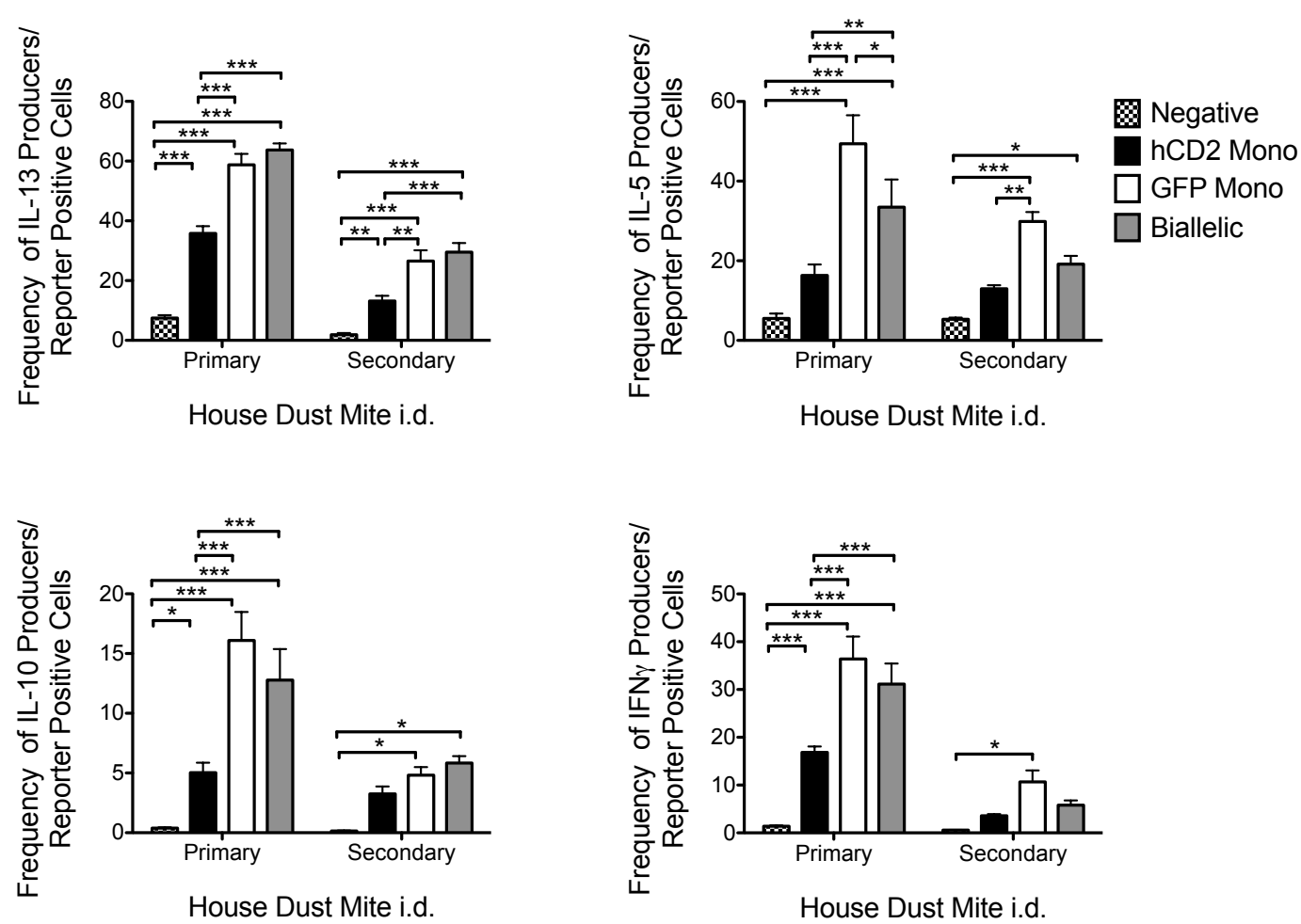

Figure 5. 4 GFP Monoallelic compared to hCD2 monoallelic reporter positive CD4 T cells are more likely to secrete a second cytokine. House dust mite was injected into the ear dermis with a challenge injection administered at 30 days; the draining lymph node immune response was tracked over time. Cytokines were determined in CD4 T cells by flow cytometry following polyclonal restimulation for $4 \mathrm{hrs}$ and intracellular staining. The frequency of dual cytokine producers of IL-13, IL-5, IL-10 and IFN $\gamma$ of CD4 T cells that were biallelic if expressing both reporters or hCD2 monoallelic or GFP monoallelic or reporter negative were compared over time. ${ }^{*} p \leq 0.01,{ }^{* *} p \leq 0.001,{ }^{* * *} p \leq 0.0001 ; 2$ Way ANOVA with a Bonferroni post test as compared to biallelic cells. Data is representative of at least 2 independent experiments with bar graphs showing mean \pm SEM for $n \geq 4$ mice per group. i.d.: intradermal. 


\subsection{Th2 cells in lymphoid and tissue environments have similar monoallelic dominant patterns of IL-4 allelic expression}

To determine whether the tissue location of the Th2 cell or type of Th2 inducing stimulus had any effect on the regulation of the expression of independent alleles of Il4, KN2/G4 mice were infected with the parasitic helminth $N$. brasiliensis. Mediastinal lymph nodes and lungs were analysed at day 9, the peak of the lung inflammatory response, and day 14 , when the Th2 response begins to diminish. $N$. brasiliensis is a potent inducer of a Th2 immune response and unlike injection of the common allergen house dust mite, the helminth infection is not confined to a single draining lymph node but instead migrates through the body creating multiple areas of antigen deposits and associated Th2 inflammation. IL- $4^{+/+}$animals were infected alongside $\mathrm{KN} 2 / \mathrm{G} 4$ (IL-4 ${ }^{--}$) animals to serve as controls for a wild type response in the presence of IL-4. The total cell counts in the mediastinal lymph node but not the lung, at both day 9 and day 14 following infection with $N$. brasiliensis, were markedly higher in the IL-4 ${ }^{++}$animals than the KN2/G4 animals (Figure 5. $5 \mathrm{~A}$ ), indicative of a role for IL-4 in the development of the Th2 response in the lymphoid compartment. The expression of IL-4 reporter proteins was tracked over time in the mediastinal lymph node and lung. At day 9, the peak of the Th2 inflammatory response, the highest frequency of IL-4 reporter positive cells per CD4 T cells was observed and this frequency dropped off slightly by day 14 (Figure 5.5 A). The expression of IL-4 in both the mediastinal lymph node and the lungs was predominantly monoallelic (Figure $5.5 \mathrm{~B} \& \mathrm{C}$ ). In summary the pattern of IL-4 allelic expression by Th2 cells was unaffected by the tissue location of the Th2 cell or the type of Th2 inducer, whether it is common allergen or parasitic helminth. 
A
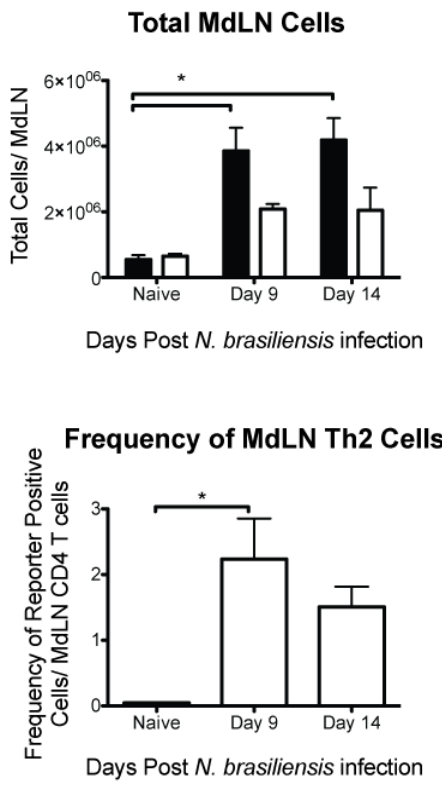

Total Lung Cells
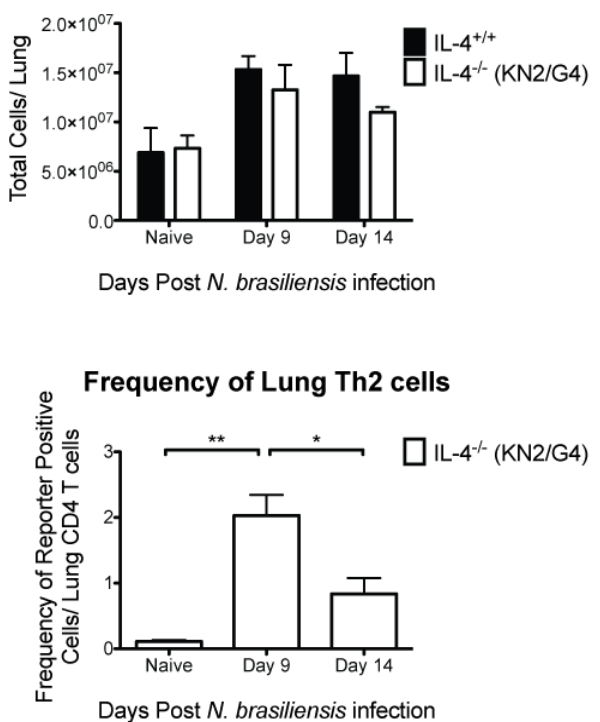

B

KN2/G4 CD4 T cells

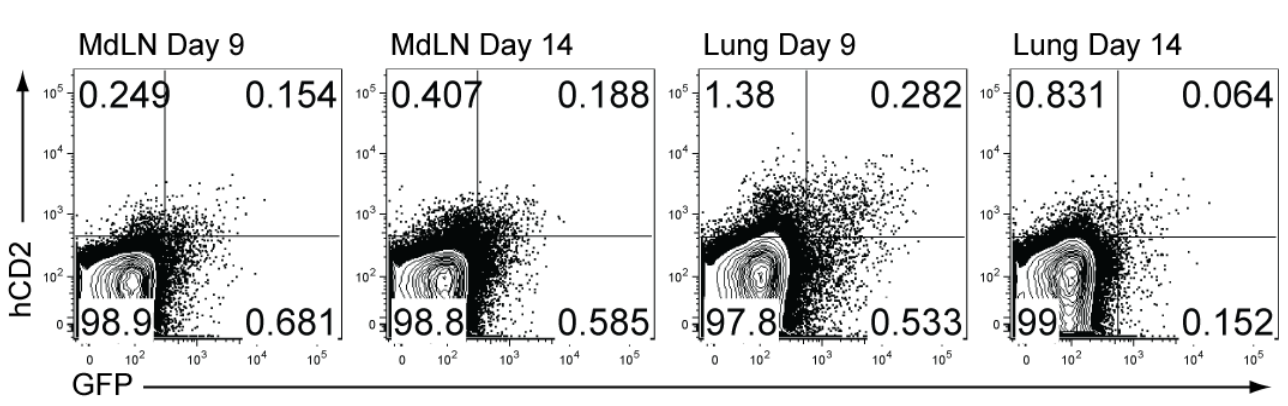

C
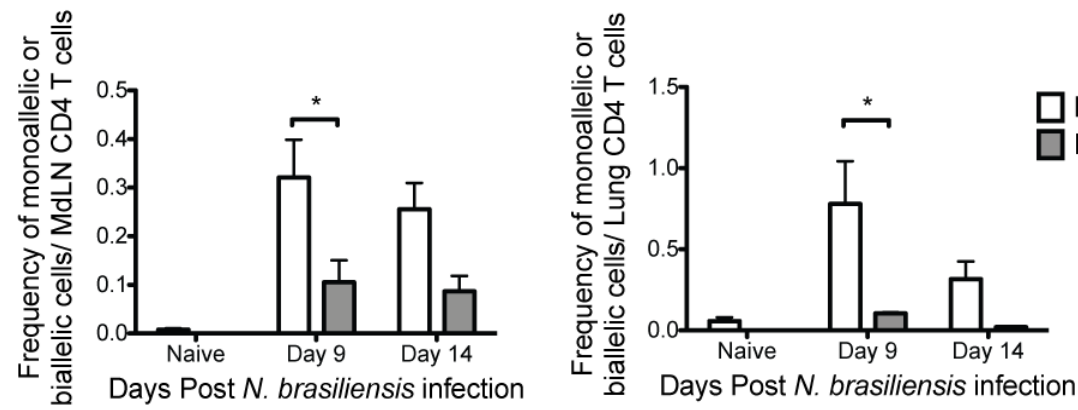

Figure 5. 5 IL-4 expression is predominantly monoallelic whether the Th2 cells are located in the lung or the mediastinal lymph node. $\mathrm{IL}-4^{+/+}$and $\mathrm{IL}-4^{-/-}$(KN2/G4) mice were subcutaneously infected with 600 L3 N. brasiliensis and 9 and 14 days later mediastinal lymph nodes and lungs were harvested, processed and $\mathbf{A}$ : analysed by trypan blue viability dye to determine live leukocyte counts and stained and analysed by flow cytometry for frequency of reporter positive cells per CD4 T cells $\underline{B}$ : Contour plots of hCD2 and GFP reporter expression on CD4 T cells indicated allelic expression at day 9 and day 14 following infection in the mediastinal lymph node and lung $\underline{C}$ : The frequency of monoallelic or biallelic reporter expression per mediastinal lymph node or lung CD4 T cells were represented graphically. ${ }^{*} \mathrm{p} \leq 0.01, * * \mathrm{p} \leq 0.001, * * * \mathrm{p} \leq 0.0001 ; 2$ Way ANOVA with a Bonferroni post test as compared to $\mathrm{IL}-4^{+/+}$animals or biallelic cells. Data is representative of at least 2 independent experiments with bar graphs showing mean \pm SEM for $n \geq 4$ mice per group. MdLN: Mediastinal lymph node. 


\subsection{Functional relevance of both alleles of IL-4 in protective immunity against parasitic helminth infections}

Protection against infection by $N$. brasiliensis is generated in the lung and is necessary for immunity against challenge infections ${ }^{161}$. This protective response is demonstrated as the significantly reduced recovery of viable worms from the lung during the challenge infection when compared to a primary infection. IL-4 is critical for efficient lung generated protective responses ${ }^{161}$. To investigate whether individual alleles of IL-4 have a role in generating this protective immunity against $N$. brasiliensis, wild type $\mathrm{IL}-4^{+/+}$, heterozygous $\mathrm{IL}-4^{+/-}$and deficient $\mathrm{IL}-4^{-/-}$animals were challenged subcutaneously with 600 live $N$. brasiliensis L3 larvae 30 days after a primary infection. Lungs were harvested two days after infection, processed in a manner to allow live L4 stage larvae to migrate out of the tissue following which the total viable worms per lung were enumerated. Following challenge infection, significantly fewer viable worms were recoverable from the lungs of $\mathrm{IL}_{-} 4^{+/+}$animals than were recoverable during the primary infection (Figure 5. 6), which demonstrates the generation of a protective immune response. IL- $4^{-/-}$animals had similar viable worm counts as those recovered during a primary infection (Figure 5. 6), indicating the complete loss of protective immunity in the absence of signalling by IL-4. The heterozygous $\mathrm{IL}-4^{+/-}$animals retained only a limited amount of protective immunity with worm burdens close to those seen during the primary infection (Figure 5. 6). These data illustrate a definitive role for two functional IL-4 alleles in generating the protective immunity required for reducing worm burdens following challenge infections with $N$. brasiliensis.

IL-4 is critical not just for the generation of protective immunity to challenge infections with $N$. brasiliensis, but also for the production of IL-4 and the presence of $\mathrm{CD} 4 \mathrm{~T}$ cells are also required for the high levels of serum $\mathrm{IgE}$ associated with helminth infections ${ }^{130,205}$. IgE production was determined at 14 days post primary infection with $N$. brasiliensis. As previously reported ${ }^{65}, \operatorname{IgE}$ titres were below detection levels in the $\mathrm{IL}_{-} 4^{--}$deficient mice. Heterozygous $\mathrm{IL}_{-} 4^{+-}$mice were able to mount an IgE response, however it was significantly reduced in comparison to the titres of IgE detectable in the serum of wild type $\mathrm{IL}-4^{+/+}$mice (Figure 5. 6). These data 
illustrate the necessity of both alleles of IL-4 in the class-switch process by B cells that leads to the production of IgE.
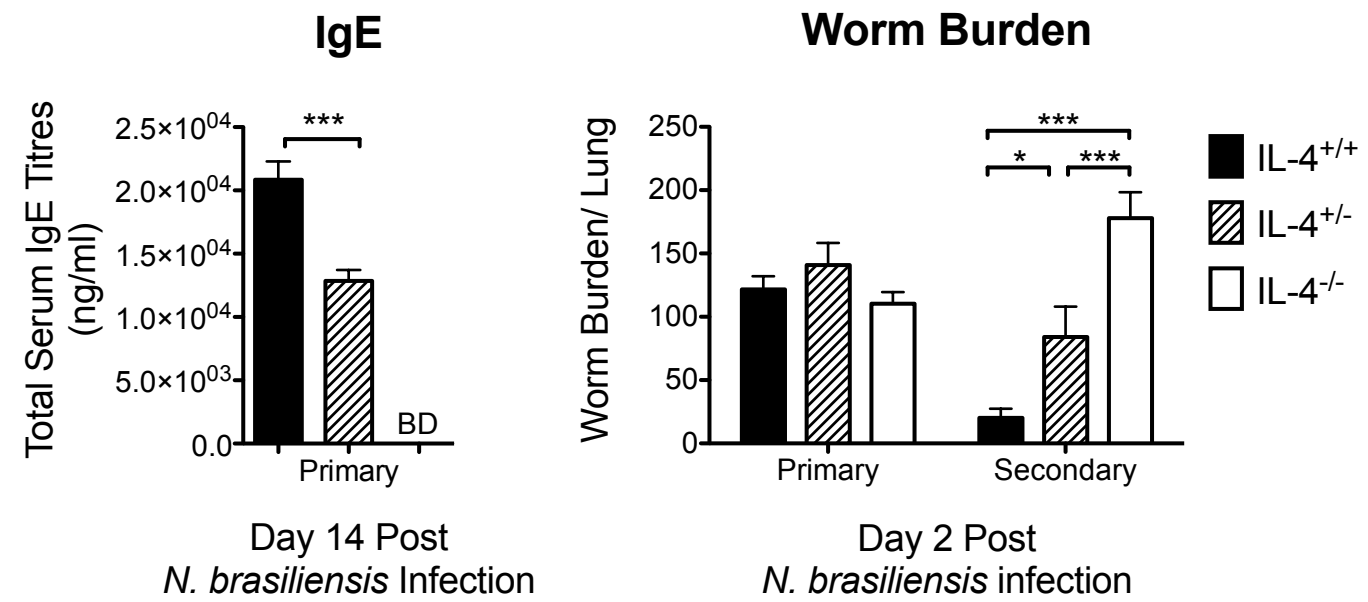

\begin{abstract}
Figure 5. 6 IL-4 heterozygous mice have a significantly impaired protective response against N. brasiliensis. $\mathrm{IL}_{-} 4^{+/+}, \mathrm{IL}-4^{+/-}$and $\mathrm{LL}-4^{-/-}$mice were subcutaneously infected with $600 \mathrm{~L} 3 \mathrm{~N}$. brasiliensis left for 30 days and challenged with same dose of parasite. Serum was obtained 14 days after primary infection and total IgE ELISAs were performed to obtain IgE titres. Live L4 worms were allowed to migrate out of lungs to determine worm burdens 2 days after primary or challenge infections. ${ }^{*} p \leq 0.01,{ }^{*} p \leq 0.001, * * * p \leq 0.0001 ; 1$ Way ANOVA with a Tukey post test. 2Way ANOVA with a Bonferroni post test as compared to all experimental groups. Data is representative of at least 2 independent experiments with bar graphs showing mean \pm SEM for $n \geq 4$ mice per group. BD: Below Detection Limit.
\end{abstract}

To confirm the finding that two intact alleles of IL-4 are required for an IL-4 mediated protective immune response against parasites, infection with the entirely enteric parasitic helminth Heligmosomoides polygyrus, which is expelled upon treatment with rIL-4, was also assessed ${ }^{217}$. IL- $4^{+/+}$sufficient, IL- $4^{+/-}$heterozygous and IL- $4^{-/-}$deficient mice were infected with $H$. polygyrus and 21 days following infection these mice were treated with an anti-helminthic to expel the worms. Fifteen days later mice received a challenge infection of $H$. polygyrus to determine whether protection remained intact in the IL- $4^{+/-}$heterozygous animals. The intestinal worm burden, egg counts and relative fecundities of worms recovered from the 3 strains of mice were enumerated and compared to that of the ${\mathrm{IL}-4^{+/+}}$sufficient primary infection. Viable 
male and female worms were allowed to migrate from the small intestine, after which they were photographed (Figure 5. 7B), enumerated and worm burdens were compared between the three strains. In comparison to the IL- $4^{+/+}$worm burdens, the $\mathrm{IL}_{-} 4^{-/}$animals had significantly higher numbers of worms after a secondary infection illustrating the loss of protective immunity in the absence of IL-4 (Figure 5. 7C). Worm burdens of $\mathrm{IL}-4^{+/-}$mice more closely resembled the $\mathrm{IL}_{-} 4^{-/-}$than the $\mathrm{IL}-4^{+/+}$ animals, illustrating the loss of even a single allele of IL-4 results in significant impairment to the generation of a protective response (Figure 5. 7C). The numbers of eggs present in the caecum was also determined and used as a measure to indicate the fecundity of the worms residing in the small intestine. Both the IL- $4^{+/}$and IL- $4^{-/}$ animals harboured worms in their small intestines that over time produced significantly more eggs and were significantly more fecund than the IL- $4^{+/+}$mice. The serum titres of IgE were significantly reduced in heterozygous ${\mathrm{IL}-4^{+-}}^{+-}$mice following infection with $H$. polygyrus, and were below detection in the IL- $4^{-/-}$deficient animals (Figure 5.7A), as seen in the N. brasiliensis infection (Figure 5. 6). Taken together, these data demonstrate the requirement for two intact alleles of IL-4 in the generation of protection from re-infection with either $N$. brasiliensis or $H$. polygyrus parasitic infections. 
A

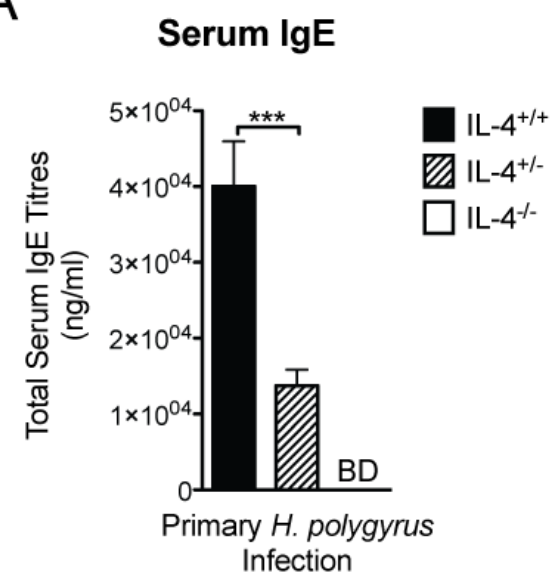

C

$$
\text { Worm Burden }
$$

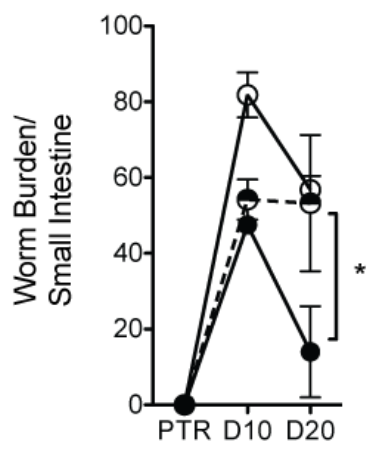

Secondary $H$. polygyrus Infection
B

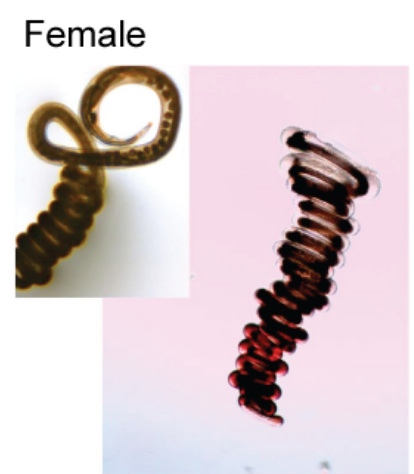

Male

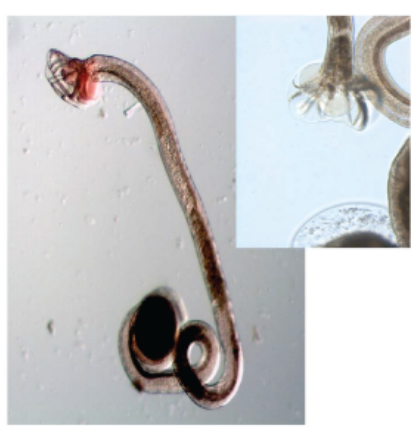

Fecundity

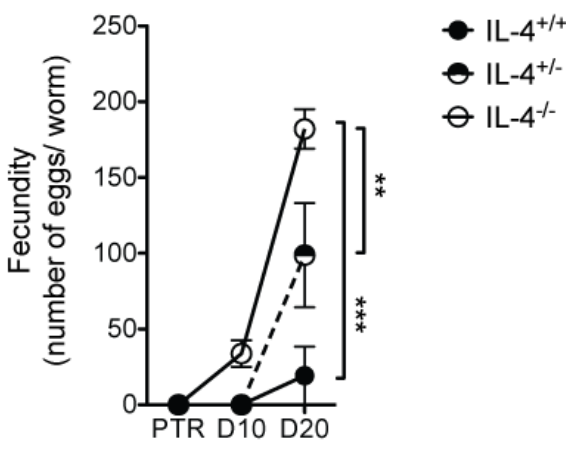

Secondary $H$. polygyrus Infection

Figure 5. 7 IL-4 heterozygous mice have significantly impaired protective responses against infection with $\boldsymbol{H}$. polygyrus. IL $-4^{+/+}, \mathrm{IL}_{-} 4^{+/-}$and $\mathrm{IL}-4^{-/-}$mice were intragastrically challenged with 200 L3 H.polygyrus left for 21 days, treated with anti-helmithic, left for a further 15 days and challenged with same dose of parasites. A: Serum was obtained 21 days after primary infection and total IgE ELISAs were performed to obtain IgE titres. B: Live L5 worms were allowed to migrate out of small intestines and photographed $\mathbf{C}$ : or enumerated to determine worm burden per small intestine at 10 and 20 days after primary and secondary infection. Eggs were obtained from contents of the caecum resuspended in salt solution and counted with McMaster counter. Fecundity was determined as numbers of eggs per numbers of viable worms. ${ }^{*} p \leq 0.01,{ }^{* *} p \leq 0.001,{ }^{* * *} p \leq 0.0001$; Mann-Whitney test. 2 Way ANOVA with a Bonferroni post test as compared to ${\mathrm{IL}-4^{+/+}}^{+}$animals. Data is representative of at least 2 independent experiments with bar and line graphs showing mean \pm SEM for $n \geq 4$ mice per group. BD: Below Detection Limit. 


\subsection{Functional relevance of both alleles of IL-4 in generating full lymphocyte compartments of the Th2 response}

The majority of IL-4 producing cells have a T follicular helper (Tfh) phenotype (PD-1 and CXCR5 surface expression) and are located in the follicular regions of the lymph node ${ }^{110,111}$. IgE class switching occurs through $\mathrm{T}$ cell help, via the engagement of CD40 ligand with CD40 and IL-4 binding through its receptor on B cells, activating transcription of the IgE heavy chain that possibly involves a follicular pathway ${ }^{126,238}$. To determine whether the significant drop in $\operatorname{IgE}$ titres observed in the heterozygous mice in Figure 5. 6 and Figure 5.7 was accompanied by a defect in the follicular compartment, the mediastinal lymph node was investigated for Tfh formation as well as numbers of $\mathrm{B}$ cells. A distinct population of $\mathrm{CD} 4 \mathrm{~T}$ cells that were expressing the Tfh markers PD1 and CXCR5 were clearly discernable in the mediastinal lymph node of wild type IL- $4^{+/+}$mice (Figure 5. 8 A). These Tfh cells were found in significantly lower frequencies in the heterozygous $\mathrm{IL}-4^{+/-}$and $\mathrm{IL}-4^{-/-}$deficient animals. Additionally, the total number of cells, the total numbers of B cells and the total numbers of Tfh cells were significantly reduced in the mediastinal lymph nodes of the heterozygous $\mathrm{IL}_{-} 4^{+/-}$and $\mathrm{IL}-4^{-/-}$deficient animals compared to the wild type $\mathrm{IL}-4^{+/+}$ animals (Figure 5. $8 \mathrm{~B}$ ). To determine whether Tfh cells were detectable within the lung, 14 days following primary infection with $N$. brasiliensis the lungs were harvested and analysed by flow cytometry for Tfh markers PD1 and CXCR5. The CD4 $\mathrm{T}$ cells in the lungs did not express Tfh markers (Figure 5. 8A) as has been reported previously by Liang et. al. ${ }^{210}$. The heterozygous IL- $4^{+/-}$and $\mathrm{IL}-4^{-/-}$deficient animals showed a trend towards fewer total leukocyte numbers per lung with significantly fewer B cells detectable in the lungs of the $\mathrm{IL}-4^{-/}$deficient animals (Figure $5.8 \mathrm{C}$ ). These data illustrate a requirement for two functional alleles of IL-4 for the Tfh and B cell compartments to effectively develop during infections with helminth parasite $N$. brasiliensis. 
A

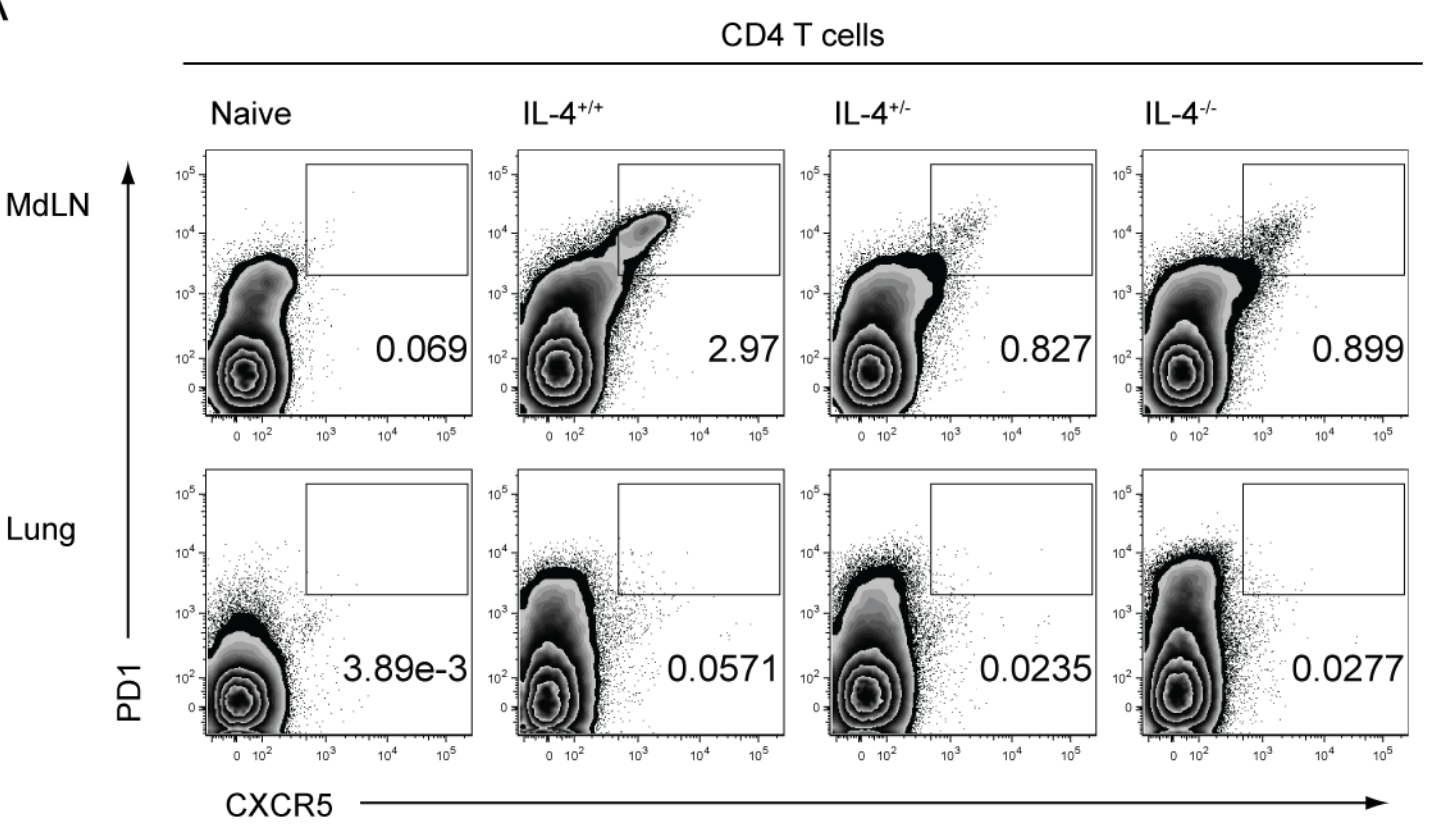

B
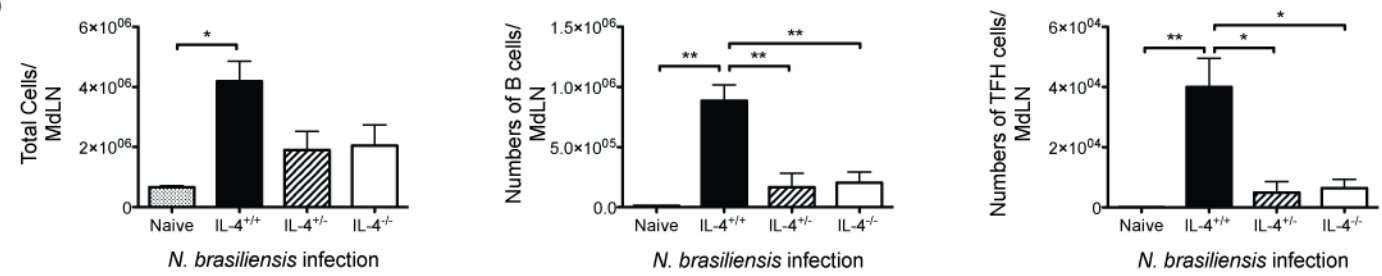

C
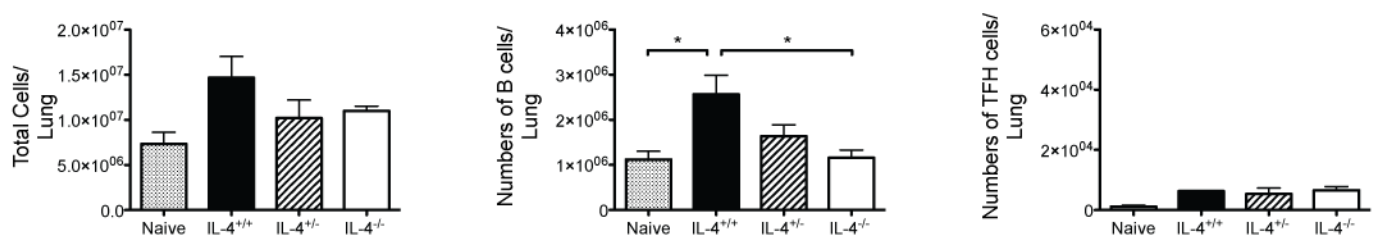

N. brasiliensis infection

N. brasiliensis infection

Figure 5. 8 IL-4 heterozygous and IL-4 deficient mice have a significantly impaired B and Tfh cell compartment during infection with $\mathrm{N}$. brasiliensis. $\mathrm{IL}-4^{+/+}, \mathrm{IL}-4^{+/-}$and $\mathrm{IL}-4^{-/-}$mice were subcutaneously infected with 600 L3 N. brasiliensis, mediastinal lymph node and lung tissues were harvested 14 days later and processed for flow cytometry. A: Tfh cells were determined as CD4 T cells which expressed both CXCR5 and PD1. B: Total cells, numbers of B cells and numbers of Tfh cells were enumerated in mediastinal lymph node C: and lung ${ }^{*} p \leq 0.01$, ${ }^{* *} \mathrm{p} \leq 0.001, * * * \mathrm{p} \leq 0.0001 ; 1$ Way ANOVA with a Tukey post test. Data is representative of at least 2 independent experiments with bar graphs showing mean \pm SEM for $n \geq 3$ mice per group. MdLN: Mediastinal lymph node. 
An entirely enteric helminth infection, $H$. polygyrus, was investigated to determine whether similar deficiencies in the follicular compartment in the lymph node were present in $\mathrm{IL}-4^{+/-}$and $\mathrm{IL}-4^{-/-}$animals in comparison to $\mathrm{IL}-4^{+/+}$sufficient controls. Following secondary infection at days 10 and 20, the mesenteric lymph nodes were harvested, processed and analysed by flow cytometry. In comparison to the $\mathrm{IL}_{-} 4^{++}$ animals the IL- $4^{-/-}$animals had significantly fewer total numbers of cells at both day

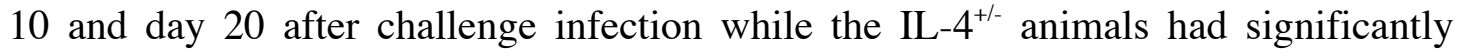
lower total cell numbers by day 20 after challenge infection (Figure $5.9 \mathrm{~A}$ ). The total numbers of B cells reflected the total cell counts per mesenteric lymph node with significantly fewer B cells in the IL- $4^{+/-}$and IL- $4^{-/-}$animals in comparison to the $\mathrm{IL}-4^{+/+}$ animals at day 20 after challenge infection (Figure 5.9 A). Unlike the total leukocyte numbers and B cell numbers, the CD4 T cell numbers were similar at day 20 between all strains, only at day 10 after challenge were there significantly fewer CD4 T cells in the IL- $4^{-/-}$animals than the IL- ${ }^{+/+}$animals (Figure 5. 9 A). This similarity in CD4 T cell number was reflected in similar numbers of Tfh cells at day 10 after challenge with significantly increased numbers of Tfh cells in the IL-4 $4^{-/}$animals at day 20 after challenge (Figure 5. 9 A). The numbers of IL-4 reporter positive CD4 T cells following $H$. polygyrus challenge infection while not significant showed a trend towards fewer Th2 cells in the IL-4 deficient animals along with significantly less GFP production per cell as measured by median fluorescent intensity at day 10 after challenge (Figure 5. 9 B). Increased worm burden in ${\mathrm{IL}-4^{-/-}}$and $\mathrm{IL}-4^{+-}$mice at day 10 post secondary infection demonstrates the necessity of CD4 T cells and IL-4 during the first 10 days of the larval stage of $H$. polygyrus infection (as seen in Figure 5.7). In summary, the mesenteric lymph nodes of $H$. polygyrus infected IL- $4^{+/-}$and IL- $4^{-/-}$ animals have significantly fewer B cells but no deficiency in Tfh cell numbers indicating that IL-4 signalling is not required for the differentiation of Tfh cells in response to this particular parasitic helminth. 
A

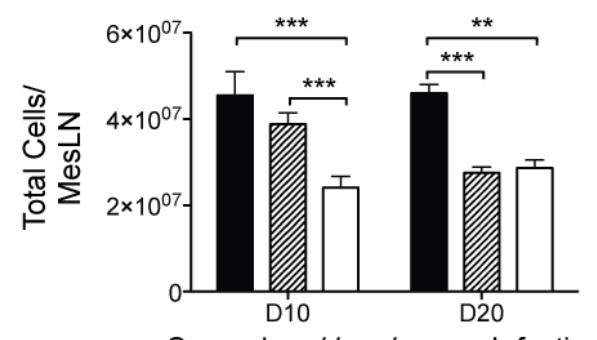

Secondary H. polygyrus Infection

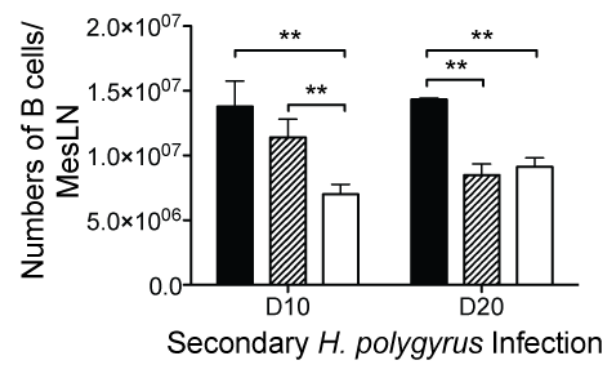

B

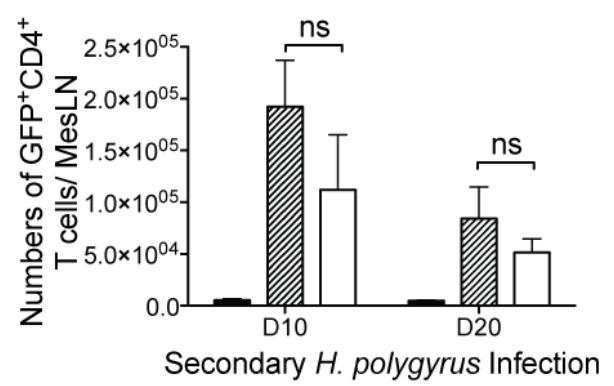

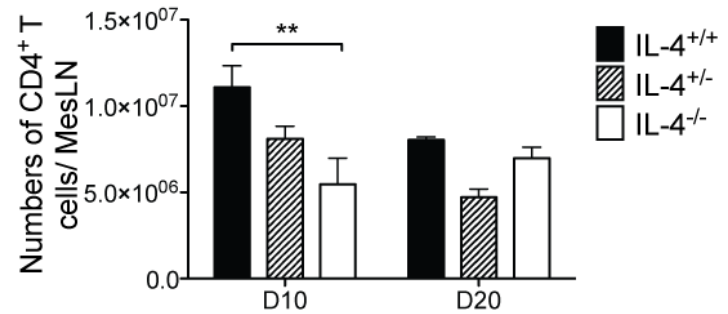

Secondary H. polygyrus Infection

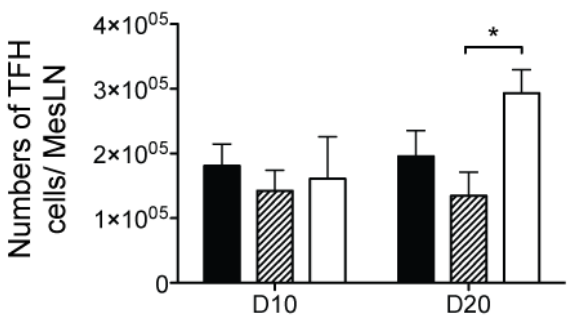

Secondary H. polygyrus Infection

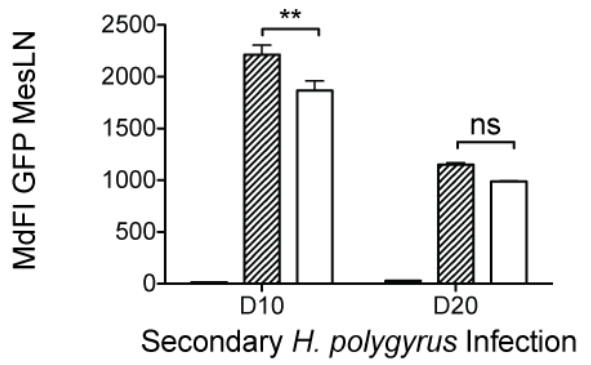

Figure 5. 9 IL-4 is required for a complete Th2 immune response against secondary infection by $\boldsymbol{H}$. polygyrus. Following primary infection and anti-helminthic treatment IL- $4^{+/+}$, $\mathrm{IL}-^{+/-}$and IL-4 ${ }^{-1-}$ mice were challenged with $200 \mathrm{~L} 3 \mathrm{H}$. polygyrus, the mesenteric lymph nodes were harvested 10 and 20 days later and processed for flow cytometry. A: The total viable cells were determined by trypan blue exclusion dye and used to enumerate the flow cytometry data to numbers of $B, C D 4 T$ and Tfh cells $B$ : as well as the numbers of GFP'CD4 T cells and the median fluorescent intensity of the GFP reporter protein. ${ }^{*} p \leq 0.01,{ }^{* *} p \leq 0.001$, *** $p \leq 0.0001$; 2Way ANOVA with a Bonferroni post test comparing all groups. Data is representative of at least 2 independent experiments with bar graphs showing mean \pm SEM for $n \geq 3$ mice per group. 


\subsection{T helper cells with biallelic IL-4 expression are more likely to be Tfh cells than the T helper cells with monoallelic IL-4 expression.}

Whether Tfh cells represent a specialised Th2 cell or an independent T helper subset, these cells have recently been described as the major IL-4 producing CD4 $\mathrm{T}$ cell subset in the lymph node of helminth infected animals ${ }^{109-111}$. To determine whether these specialised $\mathrm{T}$ helper cells also have specialised regulation of the expression of individual alleles of IL-4, the monoallelic GFP, monoallelic hCD2 and biallelic GFP were analysed to see whether monoallelic expression or biallelic expression of IL-4 enhanced the likelihood of the CD4 T cell also expressing Tfh markers. KN2/G4 mice were infected with $N$. brasiliensis and the mediastinal lymph node at both day 9 (the peak of the lung inflammatory response) and day 14 (when IgE antibodies can easily be detected in the serum) were harvested, processed and stained for Tfh markers PD1 and CXCR5. The expression of Tfh markers CXCR5 and PD1 were analysed on the GFP monoallelic, hCD2 monoallelic, biallelic double reporter positive and reporter negative cells of the CD4 $\mathrm{T}$ cell population (Figure 5. 10A). The double reporter positive biallelic cells were significantly more likely to express Tfh markers CXCR5 and PD1 than the monoallelic IL-4 expressing CD4 T cells at day 14 post infection (Figure 5. 10B). A factor to note was the increased likelihood of all reporter-positive CD4 T cells (whether monoallelic or biallelic) over reporter-negative CD4 T cells of expressing the Tfh cell surface markers. In summary, the Th2 cells with biallelic IL-4 expression are more likely to express Tfh markers PD1 and CXCR5 than the monoallelic IL-4 expressing Th2 cells or reporter negative CD4 T cells. 
A

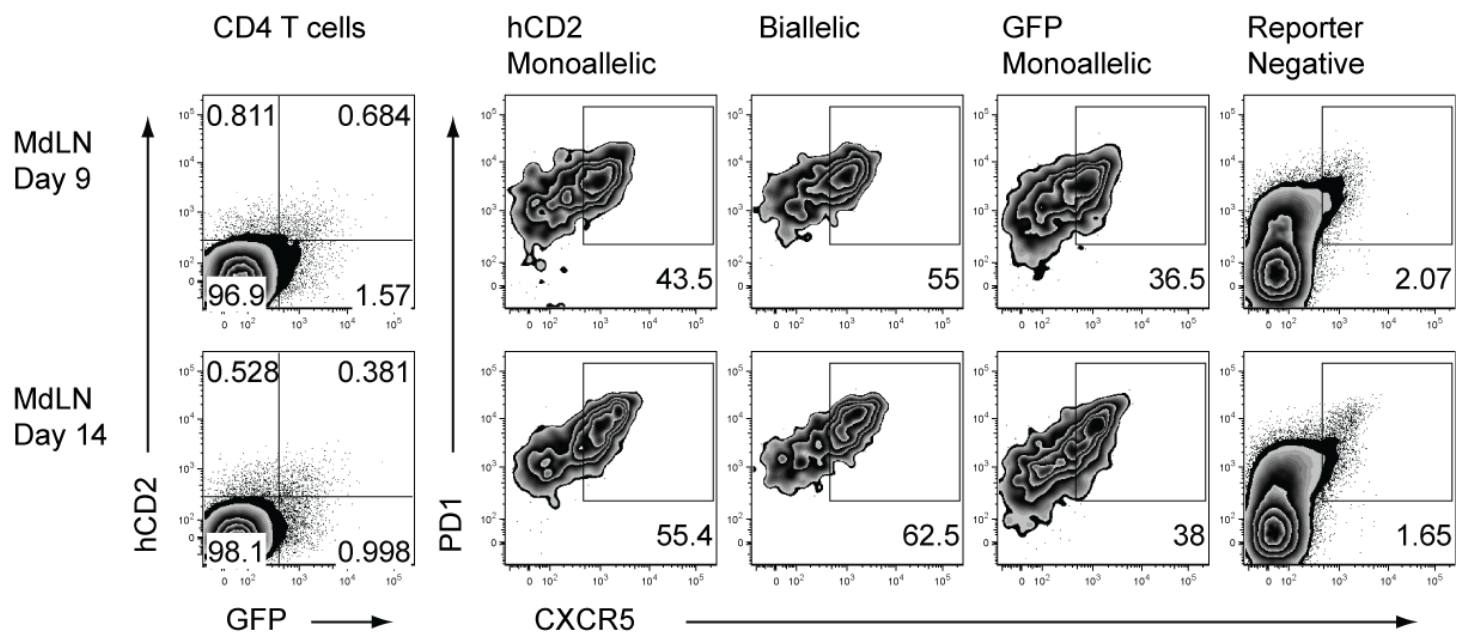

B

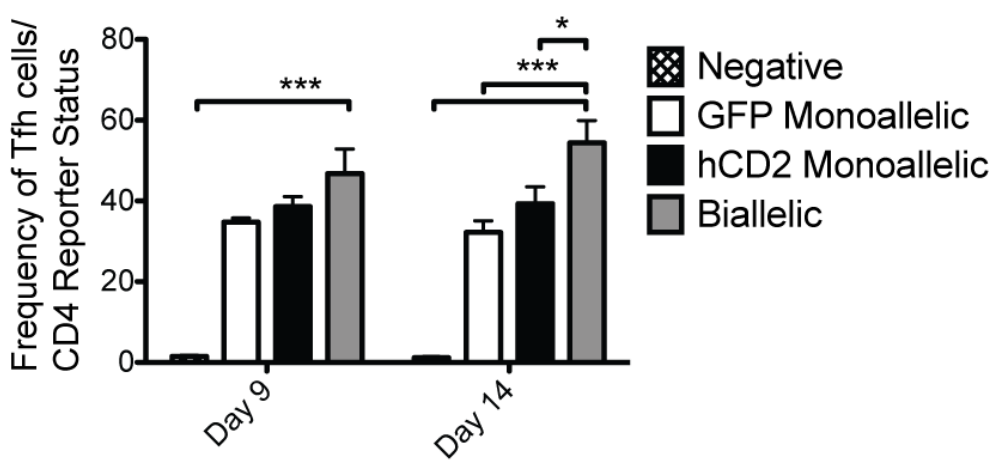

Days Post $N$. brasiliensis infection

Figure 5. 10 Biallelic IL-4 reporter positive CD4 T cells are more likely to express Tfh surface markers PD1 and CXCR5 than monoallelic IL-4 reporter positive CD4 T cells during $\mathbf{N}$. brasiliensis infection. KN2/G4 mice were subcutaneously infected with $600 \mathrm{L3} N$. brasiliensis. Mediastinal lymph nodes were harvested 9 and 14 days later. A: CD4 T cells were processed and analysed by flow cytometry for hCD2 or GFP reporter expression and gated on PD1 and CXCR5 to determine Tfh cell status. B: The frequency of Tfh cells found within the monoalleic GFP, monoallelic hCD2, biallelic GFP/hCD2 and reporter negative cells were represented graphically. ${ }^{*} p \leq 0.01, * * p \leq 0.001, * * * p \leq 0.0001 ; 2$ Way ANOVA with a Bonferroni post test as compared to the biallelic cells. Data is representative of at least 2 independent experiments with bar graphs showing mean \pm SEM for $n \geq 4$ mice per group. 


\subsection{The loss of a single allele of IL-4 compromises the numbers of IL-4 producing CD4 T cells.}

As it has previously been described that IL-4 producing Th2 cells develop and disperse to the same degree in the presence or absence or IL-4 ${ }^{65}$, two different measurements of IL-4 production were utilised to determine whether IL-4 expression was similar between the strains of IL- $4^{+/+}, \mathrm{IL}-4^{+/-}$and IL- $4^{-/-}$mice: the measurement of

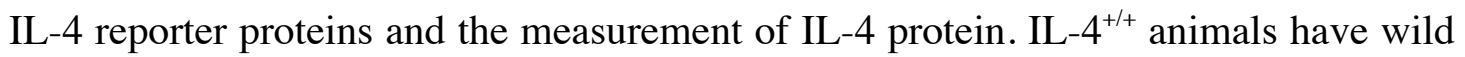
type il4 alleles whereas IL- $4^{+/-}$heterozygous and IL- $4^{-/-}$deficient animals have either a single allele of $g 4$ inserted into the il4 allele or both $i l 4$ alleles replaced with a $g 4$ and a $k n 2$ allele. These transgenic animals provided an effective tool for determining the CD4 T cell production of IL-4 as measured by the expression of the reporter proteins. Mice were infected with 600 L3 N. brasiliensis and 14 days later the mediastinal lymph nodes were processed, stained and analysed by flow cytometry for CD4 T cells and GFP-IL-4 reporter protein. The frequency of CD4 T cells was similar between IL$4^{+/+}, \mathrm{IL}-4^{+/-}$and $\mathrm{IL}-4^{-/-}$strains (Figure 5.8 B), however the total cell numbers (Figure 5. $8 \mathrm{~A}$ ) and the total numbers of CD4 T cells (Figure $5.8 \mathrm{~B}$ ) present in the lymph node were significantly lower in the $\mathrm{IL}-4^{+/-}$and $\mathrm{IL}-4^{-/-}$strains compared to the $\mathrm{IL}-4^{+/+}$ animals. When comparing IL-4 reporter positive CD4 $\mathrm{T}$ cells or Th2 cells, the frequencies, total numbers and median fluorescent intensities were similar between the IL- $4^{+/-}$and IL- $4^{-/-}$strains (Figure 5. $11 \mathrm{C}$ ). In summary, the production of IL-4 as determined by reporter protein expression is similar in the IL-4 heterozygous and the IL-4 deficient animals in response to infection with $N$. brasiliensis. 

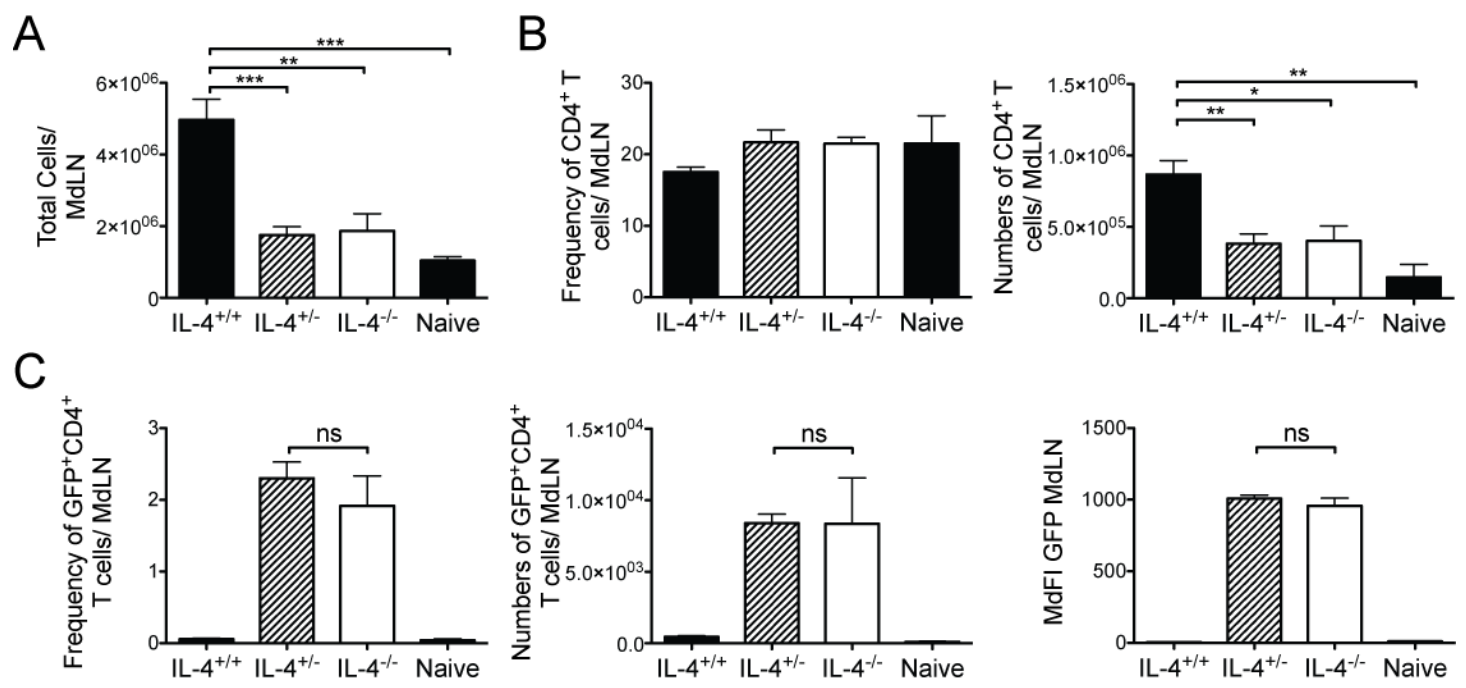

Figure 5. 11 IL-4 heterozygous and deficient mice have no difference in IL-4 reporter expression by Th2 cells during infection with $\boldsymbol{N}$. brasiliensis. IL- $4^{+/+}, \mathrm{IL}-4^{+/-}$and IL- $4^{-/-}$mice were subcutaneously infected with 600 L3 N. brasiliensis. Mediastinal lymph nodes were harvested 14 days later and processed for flow cytometry. A: Total cells were determined by trypan blue exclusion dye $\underline{\mathbf{B}}$ : Frequency and numbers of CD4 T cells $\underline{\mathbf{C}}$ : Frequency, numbers and median fluorescent intensity of GFP ${ }^{+}$Th2 cells. ${ }^{*} p \leq 0.01,{ }^{*} p \leq 0.001, * * * p \leq 0.0001 ; 1$ Way ANOVA with a Tukey post test. Data is representative of at least 2 independent experiments with bar graphs showing mean \pm SEM for $n \geq 3$ mice per group. MdLN: Mediastinal lymph node. ns: not significant.

While IL-4 reporter proteins illustrate the Th2 cells commitment to IL-4 production in IL-4 heterozygous and deficient conditions, other techniques for measuring IL-4 were employed to determine the production of IL-4 in IL- $4^{+/+}$mice. ELISPOT and ELISA techniques were used to measure the production of IL-4 protein in CD4 T cells from $\mathrm{IL}_{-} 4^{+/+}$and $\mathrm{IL}-4^{+/-}$animals. Lung CD $45^{+}$cells from day 9 N. brasiliensis infected IL$4^{+/+}$and IL-4 $4^{+-}$mice were stimulated overnight with $\alpha \mathrm{CD} 3, \alpha \mathrm{CD} 28$ and rIL-2 to induce IL-4 protein production. Following 18 hours of stimulation the lung cells from IL- $4^{+/+}$animals had significantly greater numbers of IL- $4^{+}$spots per 2 million CD $45^{+}$ cells than the lung cells from IL-4 ${ }^{+-}$animals (Figure 5. $12 \mathrm{~B}$ ). Even in cells that were not re-stimulated but left overnight to spontaneously release IL-4, the IL- ${ }^{+/+}$cells still produced significantly more IL-4 $4^{+}$spots (Figure 5. 12 A). IL-4 protein quantified by ELISA also showed a greater concentration of IL- 4 produced by the IL- ${ }^{+/+}$animals than the IL-4 ${ }^{+/}$animals (Figure 5. $12 \mathrm{C}$ ). This IL-4 concentration released into the supernatant per $2 \times 10^{6}$ cells was used to determine the concentration of IL-4 produced 
per IL- $4^{+}$cells enumerated through the ELISpot technique. A trend was noted with the $\mathrm{IL}-4^{+/-}$animals appearing to produce slightly less IL-4 per cell than that of the IL-4 ${ }^{+/+}$ animals (Figure 5.12 D). Analysis of reporter expression showed similar numbers of IL-4 producing CD4 $\mathrm{T}$ cells and similar per cell quantities of IL-4 by the IL-4 ${ }^{+/}$ animals and IL-4 ${ }^{-/}$animals (Figure 5. 11), the presence of both alleles of functional IL-4 significantly increased the total numbers of IL-4 producing cells compared to the $\mathrm{IL}_{-} 4^{+-}$animals and showed a trend towards the production of more IL-4 produced per cell in the IL- $4^{+/+}$compared to IL- $4^{+/}$animals. These data infer a positive feedback role for dual alleles of IL-4 in the development of the Th2 immune response.

A

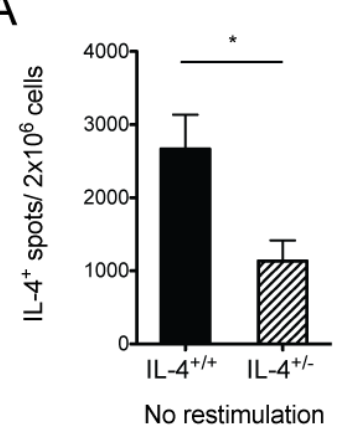

B

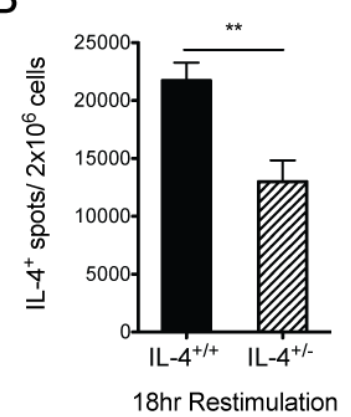

C

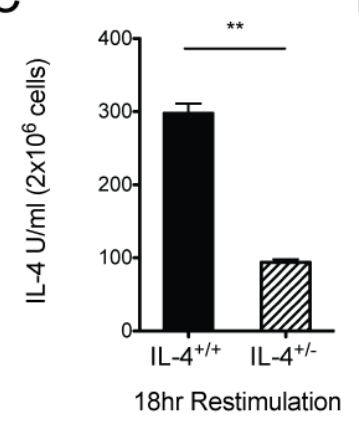

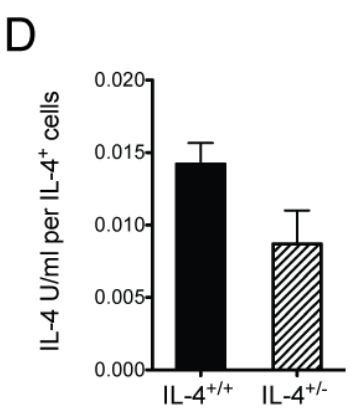

18hr Restimulation

Figure 5. 12 IL-4 wild type mice produce significantly more IL-4 secreting cells during $\mathbf{N}$. brasiliensis infection when compared to IL-4 heterozygous mice. IL- $4^{+/+}$and IL- $4^{+/-}$mice were subcutaneously infected with $600 \mathrm{~L} 3 \mathrm{~N}$. brasiliensis. Lung $\mathrm{CD} 45^{+}$cells were harvested 14 days later and IL-4 production was determined by $\underline{\mathbf{A}}$ : ELISPOT without polyclonal activation $\underline{\mathbf{B}}$ : ELISPOT with $\alpha C D 3 / \alpha C D 28$ polyclonal activation C: ELISA D: the concentration of IL-4 per IL4 producing cell was determined. ${ }^{*} p \leq 0.01, * * p \leq 0.001, * * * p \leq 0.0001$; Mann-Whitney test. Data is representative of at least 2 independent experiments with bar and line graphs showing mean \pm SEM for $n \geq 3$ mice per group. 


\subsection{Role of IL-4 in the induction of Th2 and Tfh transcription factors GATA3 and BCL6 in CD4 T cells}

To further define the role of IL-4 in developing the phenotype of Th2 cells, IL- $4^{+/+}$and $\mathrm{IL}_{-} 4^{-/}$animals were infected with $N$. brasiliensis and lymph nodes and lungs were harvested 9 days later at the peak of the lung Th2 inflammatory response, processed and intracellular stained for the Tfh transcription factor BCL6 ${ }^{101}$ and the Th2 transcription factor GATA3 ${ }^{71}$. The lung CD4 T cells from both $\mathrm{IL}_{-} 4^{+/+}$and $\mathrm{IL}_{-} 4^{-/}$ animals barely expressed BCL6 or GATA3 and were notably lower in frequency in comparison to the mediastinal lymph node cells (Figure 5.13 A\&B). Unlike in the lung, the CD4 $\mathrm{T}$ cells in the mediastinal lymph node 9 days after infection had significantly higher median fluorescent intensities and frequencies of both GATA3 and BCL6 transcription factors compared to the $\mathrm{IL}_{-} 4^{-/}$animals (Figure 5. $13 \mathrm{~A} \& \mathrm{~B}$ ). This indicated a clear phenotypic difference between activation statuses of CD4 T cells in the presence or absence of IL-4 signalling that could possibly explain the lack of Tfh formation and IgE class switching responses noted in these animals (Figure 5. 6, Figure $5.7 \&$ Figure 5.8). It is tempting to speculate that CD4 T cells from IL-4 ${ }^{+/}$ heterozygous mice would express similar levels of transcription factors to the IL- $4^{-/}$ deficient strain rather than in the $\mathrm{IL}-4^{+++}$wild type animals, but this remains to be determined. However from this data it is evident that lymph nodes undergo a significant upregulation of levels of transcription factors in the presence of IL-4 illustrating a clear role for IL-4 in the maintenance and development of a Th2 response. 
Chapter Five: Characterisation of a Th2 cell: The allelic expression of IL-4.

A CD4 T cells

MdLN

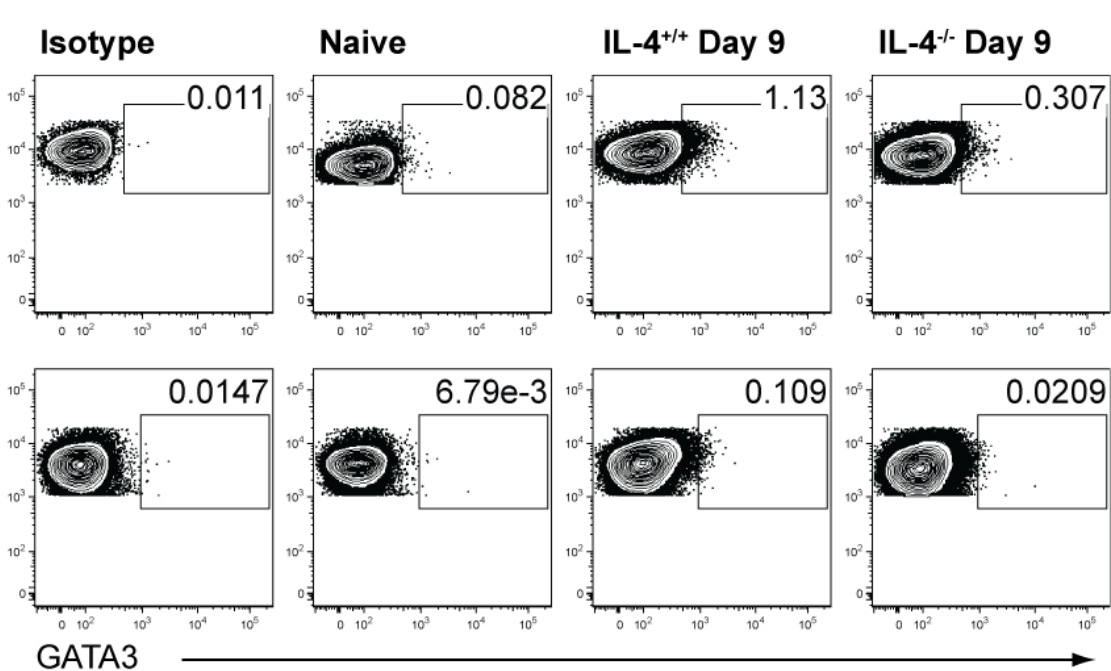

MdLN

Lung

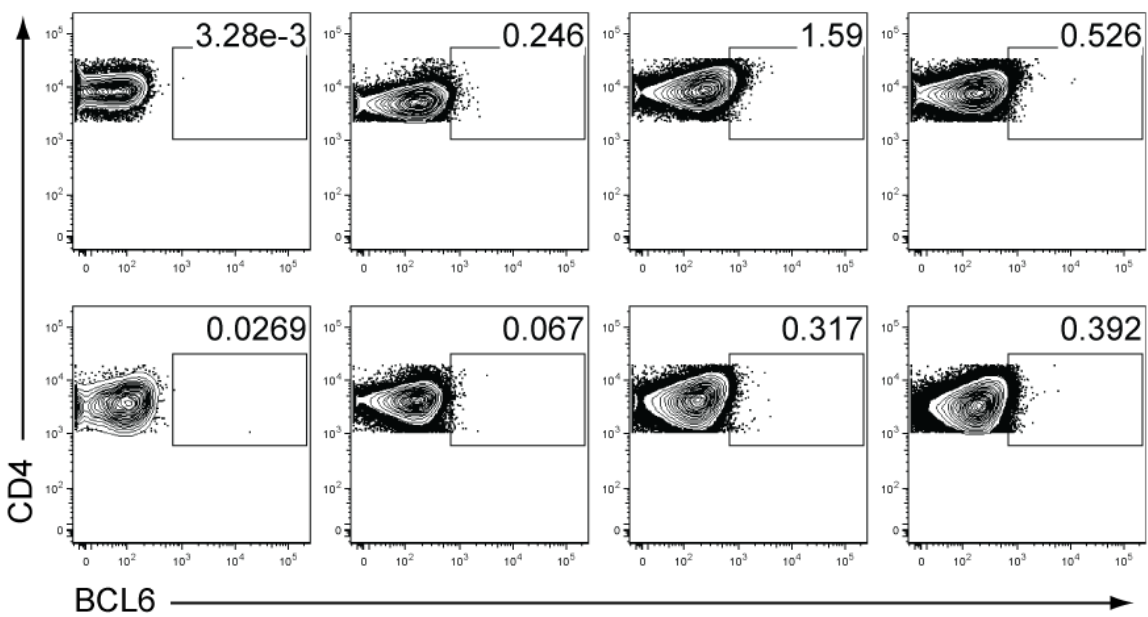

B

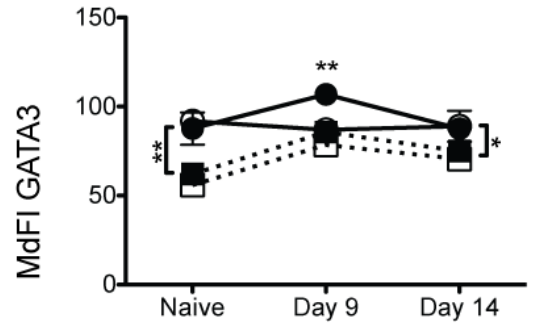

N. brasiliensis infection

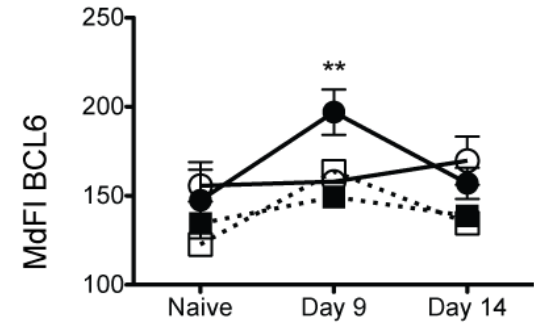

N. brasiliensis infection

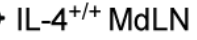
$\ominus \mathrm{IL}-4^{-/-} \mathrm{MdLN}$

IL-4 $4^{+/+}$Lung 曰 IL-4-1-Lung

Figure 5. 13 The presence of IL-4 results in significantly elevated levels of transcription factors GATA3 and BCL6 in the lymph node but not the lung. CD4 T cells from IL- $4^{+/+}$and IL$4^{-\%}$ strains of $N$. brasiliensis infected mediastinal lymph nodes and lungs were intracellular stained for BCL6 and GATA3 and analysed by flow cytometry. A: Flow plots showing BCL6 or GATA3 frequencies of CD4 T cells. B: Median fluorescent intensity of BCL6 and GATA3 in lymph nodes and lungs. ${ }^{*} p \leq 0.01,{ }^{* *} p \leq 0.001,{ }^{* *} p \leq 0.0001 ; 2$ Way ANOVA with a Bonferroni post test comparing all groups. Data is representative of at least 2 independent experiments with line graphs showing mean \pm SEM for $n \geq 3$ mice per group. MdLN: Mediastinal lymph node. 


\subsection{Discussion}

Th2 cells are characterised by their ability to produce IL-4, the hallmark cytokine of a T helper type 2 response. The production of IL- 4 by in vitro generated Th2 cells has been previously demonstrated to be predominantly monoallelic in expression ${ }^{119-121}$. This chapter investigated whether monoallelic expression occurred in vivo and whether this genetic regulation had any functional role in the development of $\mathrm{Th} 2$ immunity and could therefore be associated with functionally specialised Th2 cells. The development of a novel heterozygous IL-4 reporter mouse provided a unique tool with which to visualise the expression of individual alleles of IL-4 directly ex vivo. It was determined that the expression of IL-4 in vivo was predominantly monoallelic and this dominance was persistent regardless of the tissue location of the Th2 cell, specialised subset of the Th2 cell or Th2 inducing stimulus. There was however a small biallelic population that increased in conjunction with the increasing $\mathrm{Th} 2$ immune response displayed a more activated phenotype and was more likely to express Tfh surface markers than their monoallelic counterparts. Both alleles of IL-4 were required for protective immunity to re-infection by parasitic helminths, for effective class switching of B cells to IgE and for full differentiation of lymphoid CD4 and B cell compartments.

Due to the nature of IL-4 and its extremely short half-life it is a difficult protein to detect in vivo, the precise reason for which IL-4 reporter mice were generated. Without the use of IL-4 reporter mice, CD4 T cells are restimulated in the presence of a protein transport inhibitor to enable detection of the IL-4 protein. This is an effective but artefactual way of recording the production of IL-4 in CD4 T cells in vivo. The IL4 reporter animals overcome the short half-life of IL-4 by producing stable reporter proteins instead of unstable cytokines and allow for the measurement of IL-4 as it was expressed in situ without any need for ex vivo manipulation. The reporter proteins are not without their limitations, firstly the detection of hCD2, a surface protein, was easier to detect with certain anti-human CD2 clones than others. Secondly the two reporter proteins, GFP and hCD2, were not expressed at the same frequencies for monoallelic GFP or monoallelic hCD2 Th2 cells (Figure 5. 1 and Figure 5. 5). The reasons for this reporter expression discrepancy could be multiple and as of yet the 
cause remains uncertain, however we theorised either hCD2 requires some sort of additional support on the cell surface such as the TCR complex for stability of expression, or there is some sort of genetic regulatory factor that has been altered that associates with the $i l 4$ allele, or it is due to the half-lives of the proteins. Of note is the intron 2 regulatory site utilized by mast cells for IL-4 expression which would likely be altered in the hCD2 allele but not the GFP allele ${ }^{239,240}$, as GFP only deletes the first exon and 178nt and the first intron whereas hCD2 deletes exon 1 and 2 of the IL-4 gene ${ }^{119,190}$. Another explanation for the variability noted in the expression profiles of the hCD2 versus GFP may quite possibly be explained due to the longer half-life of GFP compared to hCD2. Following in vitro differentiation, Th2 cells were left to rest in rIL-2 $(100 \mathrm{U} / \mathrm{ml})$ where it was noted that hCD2 expression reduces to $<2 \%$ reporter expression in the CD4 $\mathrm{T}$ cell population within 2 days whereas GFP signalling takes up to 5 days to decrease to this level (Personal communication M. Robinson. MIMR, New Zealand). These data infer the GFP expression detectable during experiments performed for this chapter might be a result of earlier activation of the Il4 gene and expression of GFP protein than that of the hCD2 expressing cells. The expression of IL-4 was predominantly monoallelic with an interesting discrepancy between the GFP monoallelic cells and the hCD2 monoallelic cells where the GFP monoallelic cells were more likely to co-express an additional cytokine than the hCD2 monoallelic cells (Figure 5.4). This increased frequency of cytokine co-expression could indicate an earlier activation status of $\mathrm{GFP}^{+}$monoallelic Th2 cells compared with $\mathrm{hCD}^{+}$ monoallelic Th2 cells. This infers that these cytokine co-expressing cells may represent cells ready to exit the lymph node and enter the periphery as peripheral Th2 cells are more likely to produce additional effector cytokines than lymphoid cells ${ }^{241}$. However despite these differences in reporter protein expression, this chapter clearly showed these heterozygous $\mathrm{KN} 2 / \mathrm{G} 4$ reporter mice can be used to determine three distinct populations of CD4 T cells: monoallelic IL-4 producers, biallelic IL-4 producers and cells that do not express reporter proteins.

The biallelic IL-4 expressing Th2 cells were shown to have increased expression of the activation markers CD69 and CD44 as compared to monoallelic cells (Figure 5. 2). Additionally, CD62L and CD45RB were down regulated on the biallelic Th2 population, marking them as highly activated effector or effector memory cells. This 
highly activated effector and effector memory cell phenotype of the biallelic cells drew attention as the largest proportion of biallelic as compared to monoallelic IL-4 expressing Th2 cells was observed the day prior to challenge when the IL-4 expressing cells most likely represent a pool of memory cells lingering from the earlier exposure to house dust mite antigens (Figure 5. 1). The T helper type 2 immune response is mediated in part via cell-to-cell contact; a contact that includes CD4 $\mathrm{T}$ cell help provided to the $\mathrm{B}$ cells during class switch recombination. Therefore the quantity of IL-4 secreted per cell could affect the stimulation signal received by the target cell especially if IL-4 is passed on to the target cell through the formation of a synapse ${ }^{242}$. Experiments using the reporter mice indicate that biallelic Th2 cells produce more IL-4 on a per cell basis than the monoallelic Th2 cells (Figure 5.3) and the analysis of IL-4 protein produced by $\mathrm{IL}-4^{+/+}$and $\mathrm{IL}-4^{+/-} \mathrm{CD} 4 \mathrm{~T}$ cells indicate potentially more IL- 4 is produced by the IL- $4^{+/+} \mathrm{CD} 4 \mathrm{~T}$ cells than the IL- ${ }^{+/-} \mathrm{CD} 4 \mathrm{~T}$ cells (Figure 5. 12). Lastly the biallelic Th2 cells were shown as more likely to express a Tfh cell phenotype and therefore more likely to be found in the germinal centre than the monoallelic Th2 cells and even more so than the reporter negative cells (Figure 5. 10). This increased production of IL-4 by biallelic Th2 cells supports the theory that biallelic IL-4 expression may serve as a means for a specific specialised portion of Th2 cells to provide a stronger stimulus to the target cell.

While IL-4 may not be required to initiate Th2 differentiation ${ }^{65}$ it certainly is required for the full development of a T helper type 2 response ${ }^{20}$. This chapter highlighted the necessity for sufficient quantities of IL-4 during the maintenance of effector Th2 responses as illustrated by the loss in protection against helminth challenge infections and the drop in total IgE titres in the serum of IL- $4^{-/-}$and IL- $4^{+-}$mice (Figure 5. 6 and Figure 5.7). Although the $\mathrm{T}$ follicular helper responses were deficient in the lymph nodes of IL-4 ${ }^{+/}$and IL-4 ${ }^{-/}$animals following the brief encounter with $N$. brasiliensis (Figure 5. 8) and enhanced during the prolonged exposure to an infection with $H$. polygyrus (Figure 5.9), both infection models resulted in significant deficiencies in B cell numbers in IL-4 heterozygous mice and IL-4 deficient mice compared with the IL-4 wild type strain. The deficiency in B cells is not surprising as IL-4 was first described as a growth and proliferation factor ${ }^{18,243}$. It is interesting, however, that the IL-4 heterozygous animals show similar B cell deficiencies to the IL-4 deficient 
animals, which may indicate that the quantity of IL-4 provided by the CD4 T cell rather than the overall quantity available within the lymphoid environment, is important for B cell compartment development. A possible explanation for the difference in Tfh cell formation in the absence of IL-4 between the two parasite infections could be accounted for due to the chronic nature of $H$. polygyrus infection and the maintenance of antigen through high worm burdens in the intestines of the IL4 deficient and heterozygous animals, where alternative pathways of Tfh formation not involving IL-4 may come into play. It is tempting to further surmise that the lack of ability of individual CD4 T cells to express two alleles of IL-4 simultaneously and therefore stimulate their target cells in the appropriate manner for a fully functional Th2 immune response to occur. This theory highlights the possibility that biallelic IL4 expressing CD4 $\mathrm{T}$ cells may play an active role in the initial follicular interaction and/or the extrafollicular T cell help given to the B cells to generate IgE.

This project has clearly outlined a distinct role for signalling through both alleles of IL-4 for the generation of an effective Th2 immune response, that mediates protection from re-infection of helminths as well as inducing allergic responses. An open ended question remains as to whether this functional effect is due to a gene dosage effect or due to the lack of specialised $\mathrm{T}$ cells expressing biallelic IL-4. Unfortunately due to the current limitation of the tools available to study IL-4 allelic expression in vivo these queries cannot be answered however the highly activated biallelic Th2 cells provide an interesting target for future investigation into the genomic organisation of specialised subsets of Th2 cells. No current vaccine exists for the prevention of infection by helminths and whilst symptoms of allergic responses are treatable to an extent, there is still no cure. Both of these families of diseases lead to significant loss of quality of life resulting in major public health burdens. Moreover, hookworm immunity does not appear to be mediated by neutralizing antibodies, instead it is theorised that generation of an effective $\mathrm{T}$ cell based vaccine that generates sufficient numbers of quality long-lived effector cells would mediate protection against helminths. If biallelic Th2 cells are specialised functional cells mediating Th2 immunity, they may serve as good targets for the generation of an effective vaccine strategy against helminth infection, or as targets in immunotherapies in the prevention of allergies. 


Chapter Six:

General Discussion 


\section{1 General Discussion}

The main aim of this thesis was to expand the current understanding of the requirements for the generation and regulation of IL-4 producing T helper 2 cells. Chapter three investigated the polarisation signals required for Th2 cell differentiation with specific focus on the role of a novel Th2 innate cytokine, IL-25. Chapter four investigated whether IL-25 had any role in the circulation and maintenance of Th2 cells in both lymphoid and tissue environments and further investigated whether IL-25 was required for the generation of protective $\mathrm{T}$ helper type 2 immunity against reinfection by parasitic helminths. Finally, chapter five investigated whether the level of expression of alleles of IL-4 reflected a critical stage in genomic organisation and commitment to an in vivo functional Th2 cell.

\subsection{Investigating the potential polarising role of IL-25 during the differentiation of Th2 cells.}

The aim of chapter three was to determine whether a newly described potentially polarising cytokine, IL-25, was required for IL-4 independent differentiation of Th2 cells. To achieve this a novel in vitro antigen specific IL-4 independent culture system was developed that could detect early differentiation of Th2 cells with regards to their production of IL-4. This novel in vitro culture system was used to determine whether the maturation of bone marrow derived dendritic cells (BMDC) with rIL-25 would alter the activation phenotype of these cells and therefore increase the frequency of the differentiation of IL-4 producing CD4 T cells. The maturation of BMDCs with rIL-25 had no effect on the frequency of Th2 differentiation; this was unlike reports of TSLP ${ }^{244}$ and IL-33 ${ }^{245,246}$ matured DCs that upregulated T helper type 2 responses, although this upregulation was mostly through the production of IL-5 and IL-13 rather than IL4. Secondly the $\mathrm{T}$ cell and antigen pulsed BMDC culture system was utilised to determine whether the addition of rIL-25 would provide a polarising stimulus to the naïve CD4 $\mathrm{T}$ cell and therefore result in increased IL-4 independent Th2 differentiation. The addition of rIL-25 to the T cell cultures, however, had no effect on 
the differentiation of IL-4 producing Th2 cells. To overcome the lack of multiple additional signals provided by accessory cells, stromal cells and conditioning environment experienced in the in vitro culture system, an in vivo method was developed. This in vivo method detects early differentiated Th2 cells following exposure to a common allergen, house dust mite, or worm derived allergens from $N$. brasiliensis. Within 3 days of exposure to allergen the naive CD4 $\mathrm{T}$ cells differentiated into IL-4 producing Th2 cells, a process that occurred in both the absence of IL-25 and the dual absence of IL-25 and IL-4, illustrating no role for IL-25 during the polarisation of in vivo generated Th2 cells.

Chapter three demonstrated that IL-25 was not the innate derived polarising factor involved in IL-4 independent Th2 differentiation. The original question remains however as to what factor is responsible for the polarisation of naïve CD4 T cells into Th2 cells. It is possible different epithelial or stromal cell derived innate factor such as TSLP or IL-33 could be responsible for polarising Th2 differentiation. These cytokines are proposed to induce Th2 differentiation either through the maturation of the DC phenotype or by directly altering the naïve CD4 T cell into Th2 cells. However there is data debating the likelihood of the role of these innate cytokines (IL33 and TSLP) in Th2 differentiation. Firstly, IL-33 matured DCs induce Th2 cells to produce IL-5 and IL-13 but not IL-4, the hallmark Th2 cytokine ${ }^{245}$. Secondly, IL-33 is seldom expressed by haematopoietic cells and therefore unlikely to be expressed by DCs or other innate effector cells present in the lymph node during Th2 differentiation 247. TSLP appears a good candidate because it is produced by DCs and utilises the STAT5 signalling pathway ${ }^{248}$, a pathway that when over-expressed results in Th2 induction in a manner independent of IL-4 and STAT6 signalling ${ }^{75}$. However TSLPR I- animals show no defects in class switching of B cells to $\operatorname{IgE}$ in response to $N$. brasiliensis infection ${ }^{249}$. IgE class switching is strongly correlated with CD4 T cell production of IL-4, therefore the normal IgE titres in TSLPR ${ }^{-/}$animals imply there is no defect in the differentiation of Th2 cells in the absence of TSLP signalling. These data discussed here, while they do not directly address the action of TSLP or IL-33 on naïve CD4 T cells in vivo in the absence of IL-4, do result in sufficient queries as to whether IL-33 or TSLP present as good potential candidates for the role of polarising Th2 cells from a naïve CD4 T cell. 
It is still possible that another Stat5 activator could be responsible for IL-4 independent Th2 differentiation, as multiple cytokines are known to activate STAT5 including IL-2, IL-15 and IL-7. IL-2 could be considered a candidate for Th2 differentiation as it is produced by DCs early after activation ${ }^{250}$ and naïve CD4 T cells shortly after TCR engagement ${ }^{76}$. IL-2 would therefore be readily available to polarise a naïve CD4 $\mathrm{T}$ cell upon recognition of cognate antigen. However in agreement with previous research where IL-2 was shown to stabilise the transcription of IL-4 ${ }^{67}$, the in vitro data generated in chapter three suggests there is more to the differentiation of IL4 independent Th2 cells than a requirement for IL-2 polarisation. DC activation and the expression of specific cell-to-cell contact molecules, such as OX40L and Notch 46,244, could provide the polarising stimulus for $\mathrm{Th} 2$ differentiation and the upregulation of GATA3. Upregulation of GATA3 causes expression of IL-4 that could then act in an autocrine or paracrine manner to increase the frequency of Th2 cells ${ }^{62}$. In summary, chapter three demonstrated that IL-25 is not responsible for differentiating Th2 cells and the polarising signal remains elusive.

\subsection{The role of IL-25 in the generation of protective $T$ helper type 2 responses against infection by parasitic helminths.}

IL-25 plays a role in timely expulsion of primary infections of $N$. brasiliensis from the gut a process mediated by an IL-25 responsive lineage negative innate lymphoid cell or nuocyte ${ }^{61,175}$. It is unknown, however, if IL-25 has a role in generating memory responses to $N$. brasiliensis. Protective immunity against re-infection by $N$. brasiliensis is generated in the lungs in a CD4 T cell and IL-4 dependent manner ${ }^{161}$. The aim of chapter four was to determine whether IL-25 was required for memory responses mediated by IL-4 producing Th2 cells during the generation of protective immunity against infection with parasitic helminths. To determine whether the generation of IL-4 secreting Th2 cells was impaired in the absence of IL-25, an in vivo assay was established that enabled assessment of the differentiation and distribution of IL-4 producing Th2 cells in either the presence or absence of IL-25. The absence of IL-25 neither affected the frequency nor the numbers of Th2 cells in the lung and 
lymphoid environments immediately following infection with $N$. brasiliensis. The only differences were noted in the later time points once the Th2 inflammatory response had begun to contract, at which time significantly fewer numbers of Th2 cells were noted in the lungs in the absence of IL-25. These data support a previously described role for IL-25 in the maintenance of Th2 memory cells ${ }^{147}$ although the comparable worm burdens seen in the IL-25 sufficient and IL-25 deficient animals demonstrates that Th2 memory remains intact in the absence of IL-25. Characterisation of the Th2 population within the mediastinal lymph node and lung demonstrate signalling by IL-25 is dispensable for the dispersion and expansion of the Th2 population in both lymphoid and lung environments during an $N$. brasiliensis infection. The results generated in chapters three and four seemingly contradict earlier reports involving transgenic expression or administration of rIL-25 that resulted in exacerbated $\mathrm{T}$ helper type 2 responses ${ }^{137,138,196}$. These initial reports led to the assumption that IL-25 was responsible for the differentiation, dispersion or maintenance of Th2 cells and subsequently the type 2 immune responses. However data depicted in chapter four demonstrates that while IL-25 may play a role in maintenance of cell numbers within the lung during chronic inflammatory responses, IL-25 is not required for Th2 differentiation, dispersion, population expansion or the establishment of functional Th2 memory cells.

The absence of IL-25 signalling has been reported to skew the Th2 response towards a Th1/Th17 response following infection with the helminth Trichuris muris ${ }^{145}$. T. muris, however, is known for inducing a weaker Th2 response than those associated with both $N$. brasiliensis and $H$. polygyrus, which is associated with the inability of $T$. muris to inhibit inflammatory cytokines from dendritic cells ${ }^{249}$. The loss of IL-25 during $N$. brasiliensis infection did not result in a skewing away from a Th2 immune phenotype or deficiency in the production of T helper type 2 cytokines. Hallmarks of a $\mathrm{T}$ helper type 2 response associated with $N$. brasiliensis infection include lung eosinphilia ${ }^{251}$ and lung mucous hypersecretion ${ }^{159}$; however neither the type 2 cytokines, eosinophilia nor mucous hypersecretion were impaired in the lungs of IL25 deficient animals. An additional T helper type 2 hallmark associated with $N$. brasiliensis infections is the high titres of serum $\operatorname{IgE}{ }^{155}$, which were significantly higher in the absence of IL-25. This increased serum IgE was accompanied by 
significantly increased numbers of basophils and eosinophils in the blood. The increased numbers of eosinophils and basophils are likely to reflect the increased antigen load resulting from ongoing parasitic burden in the gut of IL-25 deficient animals. The IgE data infers IL-25 may play a regulatory role in modulating the $\mathrm{T}$ and B interactions within the lymph node although the exact nature of that regulatory role is unclear. Animals deficient in IL-21 signalling (the hallmark cytokine for Tfh cells) also have increased serum IgE titres ${ }^{133}$ and it is possible that the lack of signalling by IL-25 could result in a defect in the production of IL-21 and hence an increase in IgE 252. Due to the similarities in IL-4 producing CD4 T cell numbers in the IL-25 deficient and sufficient animals, a query is raised as to whether IL-25 is involved in either differentiating or enabling effector functions of Tfh cells within the lymph node even though it is not involved in differentiating Th2 cells. Another explanation could include an impaired regulatory $\mathrm{T}$ cell response that would thereby enable increased class switching ${ }^{253}$.

Due to the role nuocytes play in mediating primary gut expulsion of $N$. brasiliensis it was decided to investigate whether nuocytes were involved in mediating protective immunity in the lung. Lungs and mediastinal lymph nodes were analysed for the presence of nuocytes in IL-25 sufficient and deficient animals where it was noted that the absence of IL-25 had no discernable effect on the frequency or numbers of these cells. In agreement with Van Panhuys et. al. a comparison between the IL-4 reporter positive Th2 cells and the IL-4 reporter positive nuocytes confirmed that Th2 cells were the major contributors of IL-4 during secondary infections of $N$. brasiliensis ${ }^{55}$. Protective immunity is characterised by the drop in worm burden in the lungs of wild type mice following secondary infection in comparison to primary infections ${ }^{161}$. Worm burdens in the lungs of secondary infected IL-25 deficient animals were the same as those determined in IL-25 sufficient animals, illustrating no role for IL-25 in the generation of protective immunity against re-infection with $N$. brasiliensis.

Remarkably though, the expulsion of worms from the small intestines of both primary and secondary IL-25 deficient animals was not as efficient as those of IL-25 sufficient animals, demonstrating a tissue specific role for IL-25 in mediating immunity against parasitic helminth infections. The increased intestinal worm burdens were not specific 
to infection with $N$. brasiliensis as IL-25 deficient animals infected with $H$. polygyrus also had significantly increased gut worm burdens following secondary infection. These immunological differences highlighted between the lungs and the gut during the generation of a protective immune response imply different mechanisms are involved in stopping the worms in the lungs versus expelling the worms from the gut. A recent paper from Hepworth et. al. illustrate an interesting link between mast cells and early innate events one of which is the initiation of IL-25 mediated gut immunity to infection with $H$. polygyrus ${ }^{254}$. Hepworth et. al. theorises that mast cells respond to helminths by degranulating and inducing the production of the innate cytokines IL-25, IL-33 and TSLP and subsequent Th2 immunity. This mast cell mediated IL-25 induction is interesting as it could account for the differences noted between lung and gut, as during helminth infections few mast cells are detectable in the lung ${ }^{255}$ while the gut experiences mastocytosis ${ }^{256}$. A schematic illustrating the proposed roles of IL25 during expulsion of $N$. brasiliensis from the gut is depicted in Figure 6. 1. 


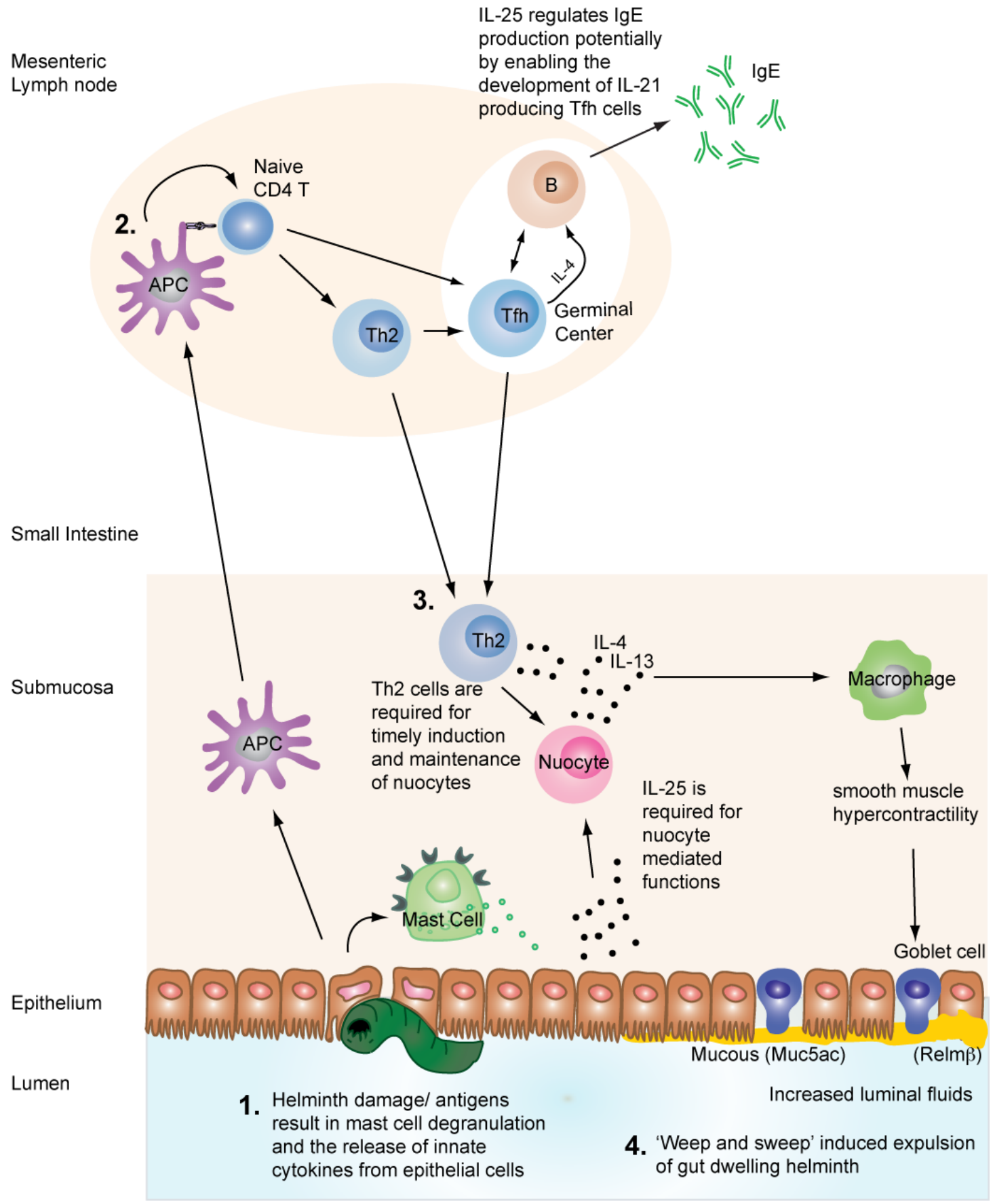

Figure 6. 1 A schematic describing the proposed roles for IL-25 during infection with gut dwelling helminthic stages of $\boldsymbol{N}$. brasiliensis. Mast cells are abundant in the gut and have the potential to act as sentinels. Upon encounter with helminth induced damage or antigens, mast cells degranulate and release signalling factors that are likely to result in the upregulation of epithelial cell production of IL-25. Antigen loaded dendritic cells differentiate Th2 cells in the draining lymph node; these Th2 cells migrate to the gut and are required for both the induction and maintenance of the nuocyte population. IL-25 has a potential role as an activation factor for the expanded nuocyte population, upon activation nuocytes secrete IL-13 resulting in the 'weep and sweep' associated mechanisms of helminth expulsion. IL-25 while not required for the differentiation of Th2 cells may be required for the differentiation of Th cells and their subsequent production of IL-21, a cytokine required for regulation of B cell IgE production. 


\subsection{Characterisation of a Th2 cell: The allelic expression of IL-4.}

The aim of chapter five was to determine whether the allelic expression of the Il4 gene in CD4 T cells could be used to provide new information on the molecular features of functional subsets of in vivo generated Th2 cells. A novel IL-4 reporter mouse was generated containing hCD2 to report one allele and GFP to report the other allele of the Il4 gene on chromosome 11 . When T cells from this mouse were stimulated to produce IL-4 it was possible to independently follow the expression of both IL-4 alleles. This allelic reporter mouse illustrated that the expression of IL-4 in vivo was predominantly monoallelic in Th2 cells. This predominantly monoallelic expression remained regardless of whether those Th2 cells were generated in response to the common allergen house dust mite or to the parasitic helminth $N$. brasiliensis, whether the Th2 cells were located in the lymph nodes or lungs and whether these Th2 cells were generated in a primary or memory response. The small population of biallelic Th2 cells expressed the highest levels of activation markers CD44 and CD69 as well as the highest levels of IL-4 reporter protein expression and the most chance of expressing $\mathrm{T}$ follicular helper markers CXCR5 and PD1. This implied these cells were a specialised subset of Th2 cells possibly ready for priming their potential cognate target cell such as a B cell or a tissue macrophage.

The effect on the generation of protective immunity in animals deficient in a single versus both alleles of IL-4 was investigated and it was discovered that the loss of a single allele resulted in significantly decreased protective immunity. The decreased lung worm burden observed in the IL-4 ${ }^{+/-}$mice during secondary infection with $N$. brasiliensis or $H$. polygyrus was similar to that seen in the $\mathrm{IL}-4^{-/-}$mice, demonstrating that the loss of a single allele is as crippling as the loss of both. This significant loss in protection was also accompanied by a significantly decreased concentration of serum IgE titres; with serum titres ranging from $2-4$ fold lower in the $\mathrm{IL}_{-} 4^{+-}$heterozygous mice compared to the IL- $4^{+/+}$sufficient animals. The lymphoid compartments in the $\mathrm{IL}_{-} 4^{+/-}$heterozygous and IL-4 ${ }^{-/-}$deficient animals were also markedly similar in their significantly lower frequencies and numbers of B cells and Tfh cells generated in response to $N$. brasiliensis infection. To recap, the allelic IL-4 reporter illustrated a predominantly monoallelic expression of IL-4 by Th2 cells but also highlighted a 
highly activated biallelic Th2 cell subset that produced more IL-4 per cell than the monoallelic cells. These data together demonstrated the production of IL-4 by a single allele was insufficient to generate a full and productive Th2 protective immune response against parasitic helminths. This discovery led to the hypothesis that the biallelic Th2 cells could represent crucial mediators of Th2 immunity possibly through cell-to-cell contact and the delivery of tailored activation signals and increased quantities of IL-4. Figure 6.2 proposes the states of genomic organisation reflected by the levels of expression of alleles of IL-4 associated with specialised subsets of Th2 cells mediating immunity in vivo.

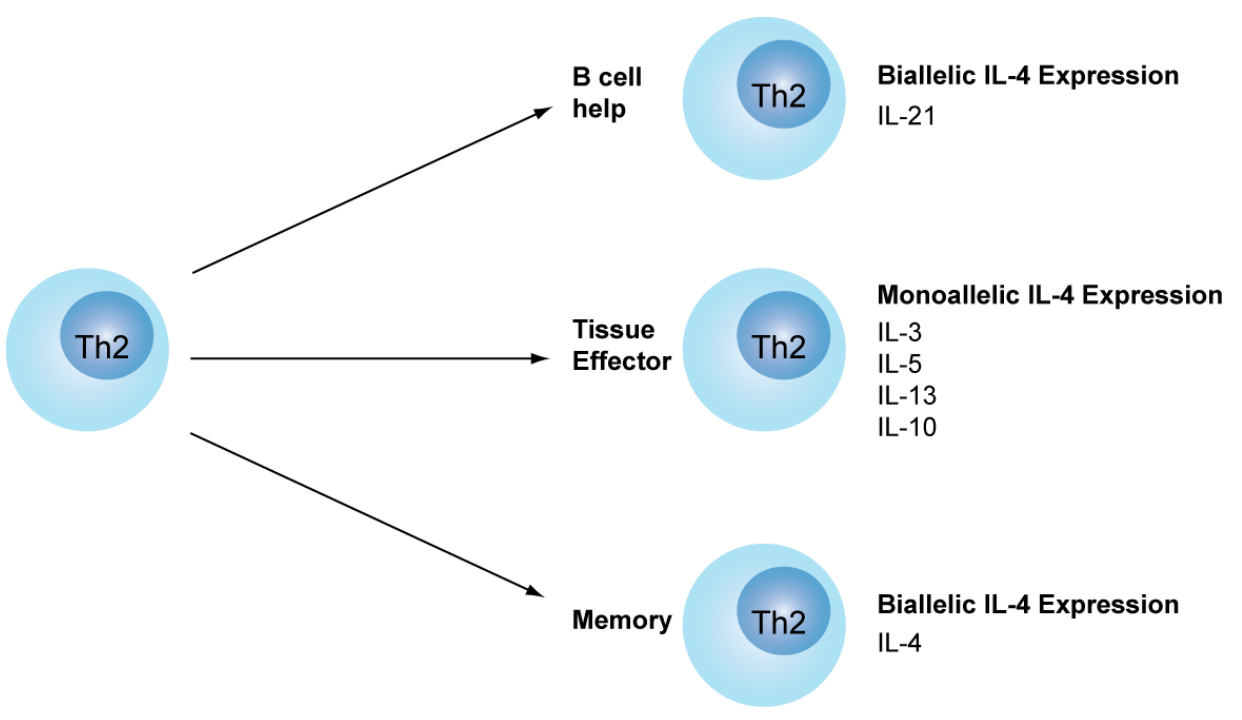

Figure 6. 2 The regulation of IL-4 through the monoallelic or biallelic expression of the il4 alleles is associated with functional subsets of Th2 cells. Biallelic expression of IL-4 by Th2 cells is associated with T follicular helper cell specialisation and the provision of B cell help as well as the establishment of a long lived and rapid reacting memory Th2 cell. Monoallelic expression of IL-4 is associated with effector Th2 cells located in the tissues which are required for mediating $\mathrm{T}$ helper type 2 responses such as eosinophilia or mucous production. 


\subsection{Understanding the generation and regulation of IL-4 producing Th2 cells.}

Traditionally the subsets of $\mathrm{T}$ helper cells were characterised by the cytokine they produce and the functional effect that ensues, e.g. IL-4 the 'signature or hallmark' cytokine of Th2 cells ${ }^{6}$. Advances in molecular biology resulted in the discovery of particular transcription factors involved in the differentiation of the $\mathrm{T}$ helper subsets with GATA3 recognised as the Th2 signature transcription factor ${ }^{70,71}$. More recently developments in the field of epigenetics revealed certain chromatin states stabilise particular cell phenotypes, a status that can be modified in the expanding population through environmental signals such as cytokines or stress factors ${ }^{257,258}$.

The original subset definitions occurred largely through analysis of in vitro generated $\mathrm{T}$ helper cell subsets. These in vitro culture systems are widely recognised as excluding of multiple environmental factors that the helper $\mathrm{T}$ cell would be exposed to in vivo. The traditional method of in vitro differentiation of a Th2 cell using polyclonal stimulation along with rIL-2 and rIL-4 drives a highly polarised phenotype, with notably higher frequencies of IL-4 production, uniform increases of GATA3 and the expansion of the physical size of the Th2 cell in comparison to that of a Th2 cell generated in vivo ${ }^{8}$. Data on Th2 cell differentiation in vivo highlights the simplicity of this in vitro model with the discovery of the differentiation of Th2 cells in the absence of IL-4 signalling ${ }^{65}$. More recently IL-4 producing lymph node situated CD4 T cells expressing IL-4 through transcription factors independent from that of 'conventional Th2 cells' have been described ${ }^{111,117,118}$. From data such as this it is clear that Th2 cells are not a homogenous population and while Th2 cells may be classified by the production of IL-4 their location and additional cytokine repertoire or co-stimulatory molecules will contribute to their independent functional effects.

In the future as techniques such as intravital microscopy improve, hopefully the analysis of the Th2 cell in situ, where it is regulated by the full contingent of the microenvironment, will result in a much clearer understanding of what it is to be a Th2 cell and what this means for disease outcomes. Such techniques may help clarify whether we should consider IL-4 producing CD4 T cells in the lymph node as Th2 
cells or Tfh cells. It remains to be determined whether IL-4 is the hallmark Th2 cytokine in vivo, or if it earned its status through in vitro culture systems that are not true reflections of an in vivo Th2 immune response. In addition, it is important to determine whether the 'conventional Th2 cell' is a cell that has the ability, according to the stimulus encountered, to secrete multiple cytokines or the plasticity to alter the cytokines secreted e.g. evolving from IL-4 production to IL-9 production. Hopefully as fate-mapping techniques improve these sorts of questions will be answered in situ in physiologically relevant models of infection and allergy. Additional queries such as whether the germinal centre Tfh cell has a role to play in the formation of memory Th2 cells or whether antigen cognate effector T cells participate in the development of follicular and extrafollicular plasma cells also requires investigation. The possibility that non- germinal centre 'conventional Th2 cells' make up rapid lines of defence while 'Tfh/Th2 cells' give CD4 T cells time to affinity mature in response to antigen presented on B cells or plasma cells and subsequently form longer-lived higher affinity central memory or effector memory subsets also merits study. 


\section{2 General Conclusions}

In conclusion, this thesis demonstrated no role for IL-25 in the differentiation, expansion or development of Th2 cells. It further highlighted that while IL-25 is not required for Th2 lung inflammation associated with infection by $N$. brasiliensis it is also not required for the generation of protective immunity against re-infection by $N$. brasiliensis. IL-25 is however required for the timely expulsion of helminths from the gut which highlights a tissue specific role for this innate cytokine. The functional Th2 immune response that occurred in the absence of IL-25 signalling raised questions concerning the signalling roles assigned to various cytokines as the result of models using physiologically irrelevant amounts of recombinant cytokine or incomplete in vitro models of immune responses. While IL-25 had no particular role in the differentiation or development of Th2 cells or the generation of memory responses against $N$. brasiliensis, a role for IL-25 in the exacerbation of Th2 immune responses cannot be ruled out. A distinctive role for IL-25 in the gut was demonstrated in this thesis with similar reports attributing this role as IL-13 mediated, highlighting a potential role for IL-25 oral allergen-induced diarrhoea ${ }^{259,260}$ and quite possibly foodinduced allergic reactions. Lastly this thesis demonstrated the expression of alleles of IL-4 in in vivo Th2 cells was predominantly monoallelic. There was however a small biallelic IL-4 expressing population that was highly activated and more likely to coexpress Tfh surface markers, a population that was proposed to mediate specific functional effects including the class switch of B cells to IgE. As techniques advance and our understanding of the genomic organisation of a Th2 cell improves, hopefully clearer definitions of specialised Th2 subsets within the Th2 population will enable tailored approaches for treatment strategies against allergic and parasitic diseases. 


\section{3 Future Directions}

The factor or factors responsible for the differentiation of Th2 cells remain elusive and may remain elusive until such time as better tools are generated for answering this question. The in vitro antigen specific culture system generated and reported in chapter three illustrates the necessity of IL-4 for full differentiation of frequency and phenotype of Th2 cells in vitro. It is possible that a more realistic microenvironment similar to that experienced in vivo could be achieved by the addition of other factors such as lymph node stromal cells or allergen-activated epithelial cells thereby enabling the teasing out of the elusive Th2 polarising factor/s. Once potential candidates are established and genetically modified animals are developed with these potential candidates deleted, the use of the single draining lymph node assay in the IL4 deficient G4 reporter mice provides an effective tool for clearly visualising these early IL-4 independent differentiation events.

Another possibility for why the factor or factors responsible for Th2 differentiation remain elusive is that the question is phrased incorrectly. The question of whether IL25 is the polarising factor arose because there is the belief that the cytokine milieu (generated one would suppose by the antigen presenting cell) plays a crucial role in differentiating $\mathrm{T}$ helper cells into their distinctive subsets. However only the differentiation of the Th1 cell subset really follows the principle of requirement for a particular cytokine milieu i.e. IL-12 produced by dendritic cells. Instead, it is possible that the cytokine milieu in the lymph node is not the polarisation factor required for naïve CD4 $\mathrm{T}$ cells to differentiate into Th2 cells. A Th2 response is a dangerous response with the constitutive expression of IL-4 resulting in lethal phenotypes ${ }^{261,262}$. It is understandable that Th2 differentiation would not rely merely on the production of a soluble molecule that could disperse across the lymph node but instead rely upon a cell-to-cell contact molecule that requires the dendritic cell to come into contact with the naïve CD4 $\mathrm{T}$ cell. The upregulation of notch or OX40L by dendritic cells conditioned in environments driven by factors such as the innate cytokines TSLP and IL-33 are good examples of this highly specialised dendritic cell that preferentially drives Th2 differentiation. A proposal for exciting future prospects for Th2 polarising factors would be to define the transcriptomes of in vivo generated DCs presenting 
antigen that results in Th2 immune responses in comparison to DCs resulting in Th1 responses attempting to tease out the cell surface molecules that engage and differentiate the naïve CD4 T cell.

An interesting discrepancy arose as to how IL-25 mediates expulsion of the helminth parasites from the gut while it has no apparent role in the lung. Firstly the use of IL-13 reporter animals would allow for the easy monitoring of the nuocyte population and determine if in the absence of IL-25 signalling, the nuocyte population is present but merely not activated and therefore not producing IL-13 resulting in a lack of expulsion. Secondly the link between early mast cell activation by helminths in the gut and the induction of IL-25 could be investigated. If these mast cells are crucial initial mediators required to degranulate prior to IL-25 release then one would expect to see early mast cell degranulation high levels of IL-25 and rapid N. brasiliensis expulsion from the gut in IL-9 transgenic mice. Of course experiments with transgenic animals can be of limited use because they often express non-physiologically relevant levels of cytokine that result in excessive innate responses not normally associated with parasitic infection. Therefore mast cells and IL-25 production should also be measured in the gut of wild type versus IL-25 deficient animals to determine whether mast cells do act as sentinels and degranulate and activate epithelial cells inducing the production of IL-25. This data may help to explain a previous study which shows mRNA IL-25 levels in the gut only rise around 7 days following primary infection ${ }^{136}$.

An additional unanswered question in chapter four is the high levels of serum $\operatorname{IgE}$ in the IL-25 deficient animals. Of course the CD4 T cells within the lymph node could be producing sustained IL-4 as a result of continued worm burdens or IL-25 could serve a purpose in the development of the $\mathrm{T}$ and $\mathrm{B}$ interactions that result in $\operatorname{IgE}$ class switching. A lymph node phenotype of the IL-25 deficient animals including GC B cells, Tfh cells, BCL6 and the production of IL-21, GC histology, total serum IgG1 titres as well as high affinity IgG1 and IgE antibodies in the absence of IL-25 would give an overview of any major changes.

Chapter five raised the fascinating query as to whether the biallelic expression of IL-4 could constitute specialised and functionally distinct Th2 cells. It is of interest to 
determine if these biallelic Th2 cells are the true mediators of Th2 memory or if they are responsible for IgE class switching. If the biallelic Th2 cells can be defined through surface markers such as PD1, ICOS and CD40L then perhaps Th2 cells generated from an $N$. brasiliensis infection could be generated in IL-4 sufficient animals, cell sorted for 'biallelic activation markers' and adoptively transferred into IL-4 deficient animals. These animals with adoptive transferred cells could be challenged with $N$. brasiliensis and worm burdens determined. Or as a more direct readout of cell-to-cell contact rather than concentration of IL-4, the biallelic cells could be compared in vitro to monallelic cells to determine their IgE class switching efficiencies. Of course the expression of IL-4 would need to be confirmed as biallelic through a technique such as FISH analysis of monoallelic expressed genes ${ }^{263}$.

A limit to the current allelic system is the lack of IL-4 in the KN2/G4 mice. A major question remains as to whether in an IL-4 sufficient condition the biallelic population would increase significantly. It remains to be seen if the presence of IL-4 would significantly alter the biallelic expression of the reporter proteins. If this is the case then the proportion of biallelic expressing Th2 cells in wild type animals may be far larger than those accounted for in the absence of IL-4 in KN2/G4 animals. 


Appendix: 

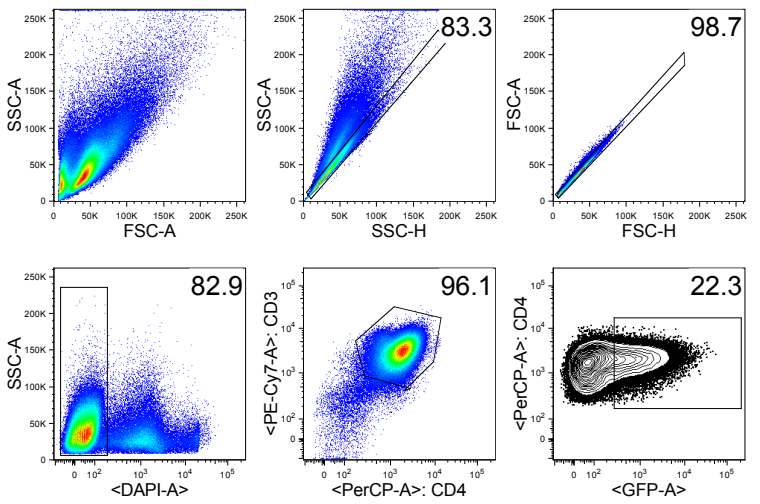

Figure A. 1 The FACS plots are read from left to right and represent the hierarchical gating strategy used for analysing Th2 cells. (Doublet', Live (DAPI'), $\mathrm{CD3}^{+}, \mathrm{CD4}^{+}, \mathrm{IL}^{-} 4$ Reporter GFP ${ }^{+}$)
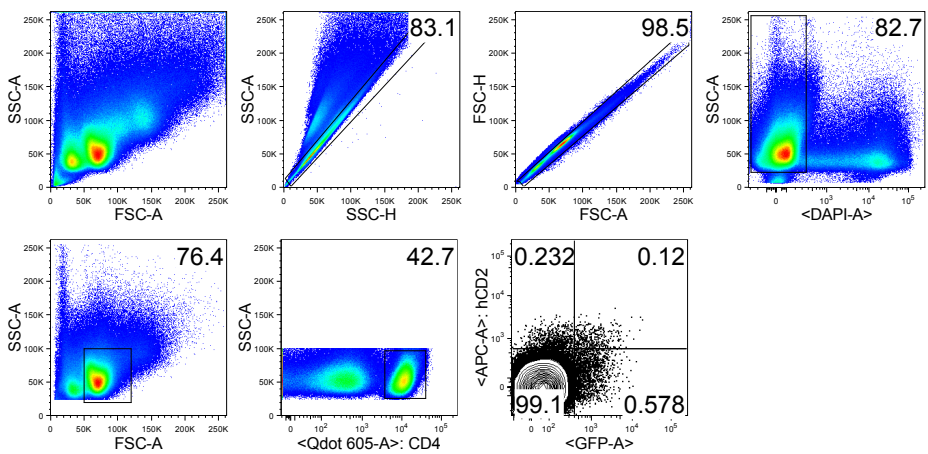

Blue: Naive CD4 T cells

Red: hCD2 monoallelic reporter positive CD4 T cells
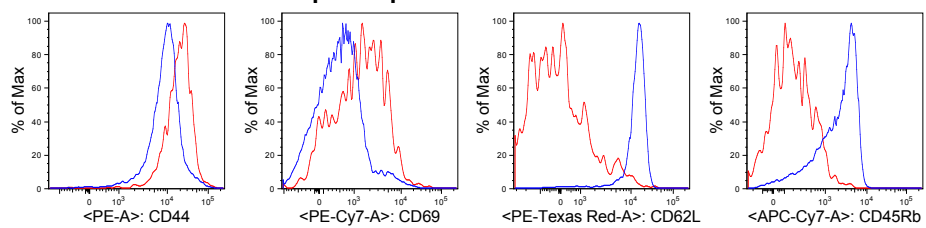

Figure A. 2 The FACS plots are read from left to right and represent the hierarchical gating strategy used for analysing the allelic expression of IL-4 by Th2 cells. (Doublet,' Live (DAPI'), Lymphocyte gate, $\mathrm{CD}^{+}$, IL-4 Reporter $\mathrm{GFP}^{+}$versus IL-4 Reporter $\mathrm{hCD2} 2^{+}$) The histograms of representative plots showing expression of activation markers, naïve CD4 T cells (blue) versus hCD2 reporter positive CD4 T cells (red) illustrate levels of expression of activation markers CD69, CD44, CD62L, CD45RB. 
Appendix:
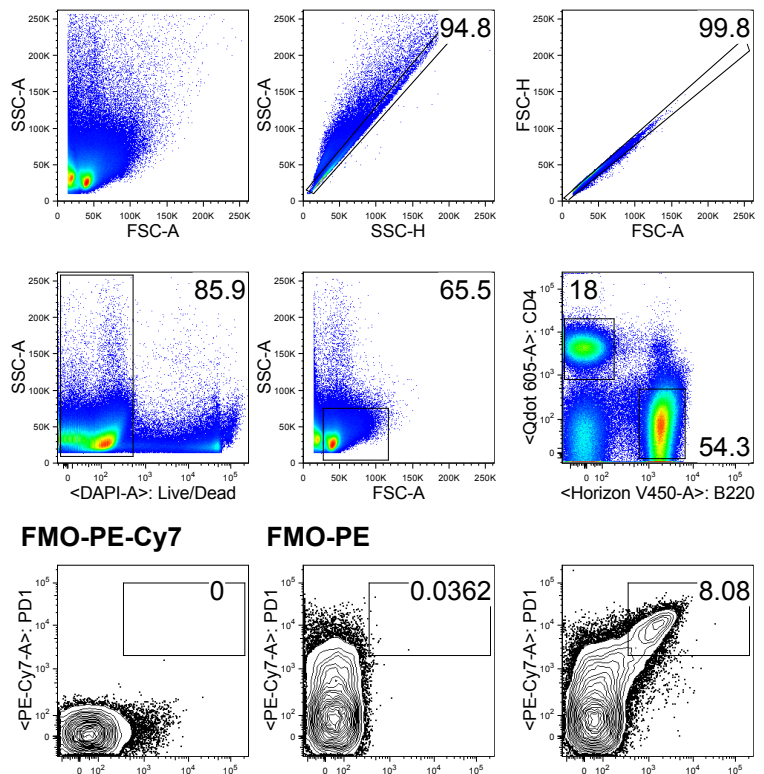

FMO-PE
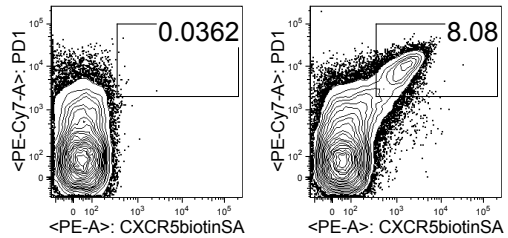

Figure A. 3 The FACS plots are read from left to right and represent the hierarchical gating strategy used for analysing $T$ follicular helper (Tfh) cells. (Doublet, Live (DAPI'), Lymphocyte gate, $\mathrm{CD}^{+} \mathrm{B} 220^{-}$, FMO-PE-Cy7 and FMO-PE gating controls for $\mathrm{PD}^{+}{ }^{+} \mathrm{CXCR5^{+ }}$ )
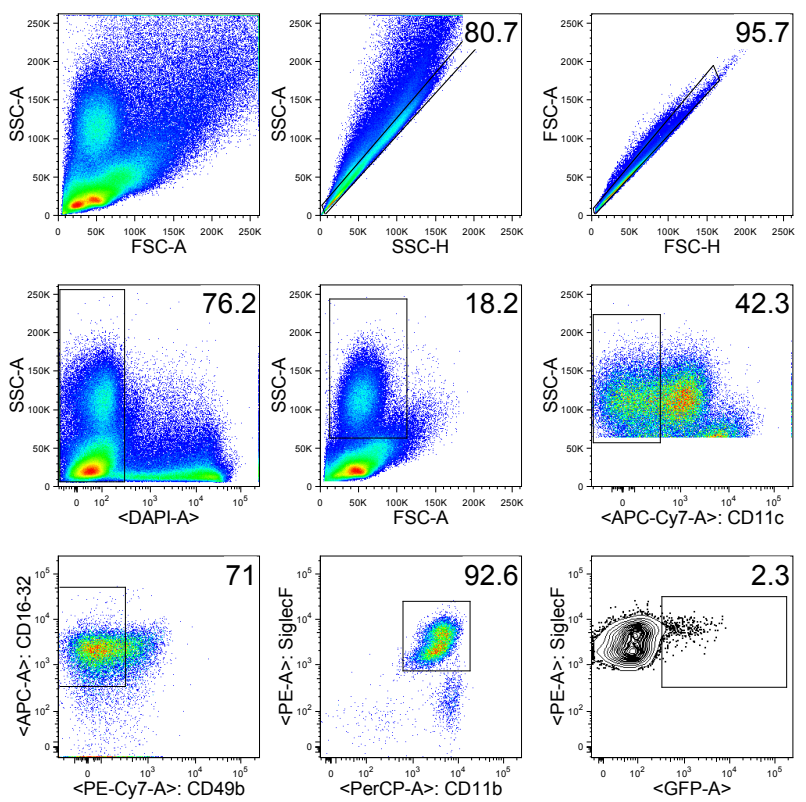

Figure A. 4 The FACS plots are read from left to right and represent the hierarchical gating strategy used for analysing IL-4 $4^{+}$eosinophils. (Doublet', Live (DAPI)), SSC ${ }^{\text {hi }}, \mathrm{CD}^{-} 11 \mathrm{C}^{-}, \mathrm{CD} 16 / 32^{+}$, CD49b (DX5) , CD11 b ${ }^{+}$, SiglecF ${ }^{+}$, IL-4 Reporter GFP ${ }^{+}$) 
Appendix:
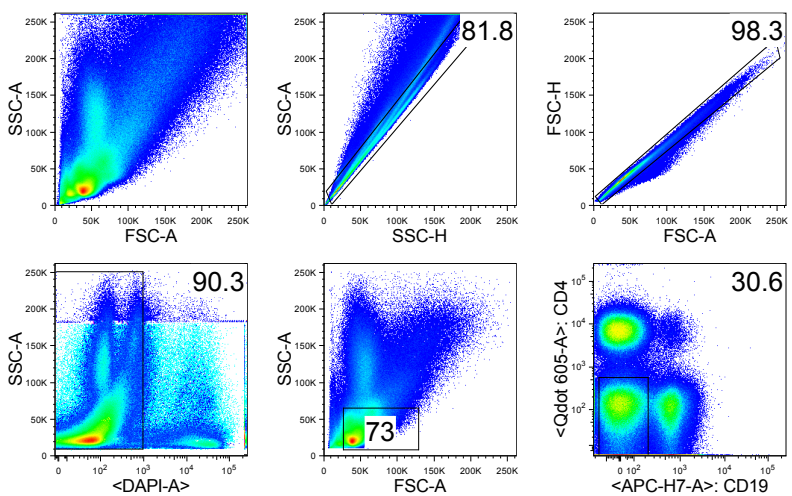

FMO-PE-Texas Red
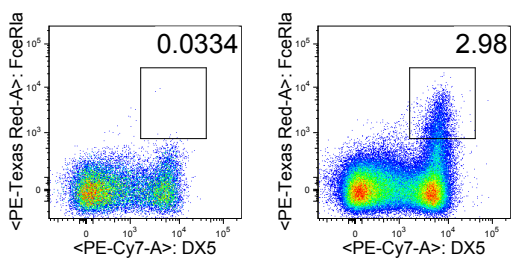

FMO-APC
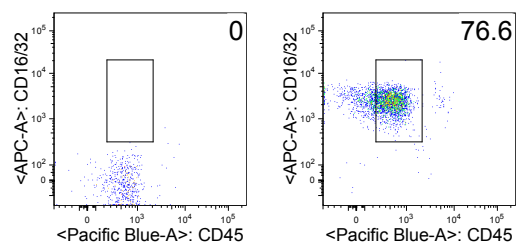

Figure A. 5 The FACS plots are read from left to right and represent the hierarchical gating strategy used for analysing basophils. (Doublet', Live (DAPI), non-B non-T cells (CD4 ${ }^{-} \mathrm{CD}^{-} \mathrm{9}^{-}$), FMO-PE-Texas Red gating controls for FceR1 $\alpha^{+}, \mathrm{CD} 49 \mathrm{~b}(\mathrm{DX} 5)^{+}, \mathrm{FMO}-\mathrm{APC}$ gating controls for $\mathrm{CD} 16 / 32^{+}, \mathrm{CD} 45^{\text {int }}$ ) 
Appendix:
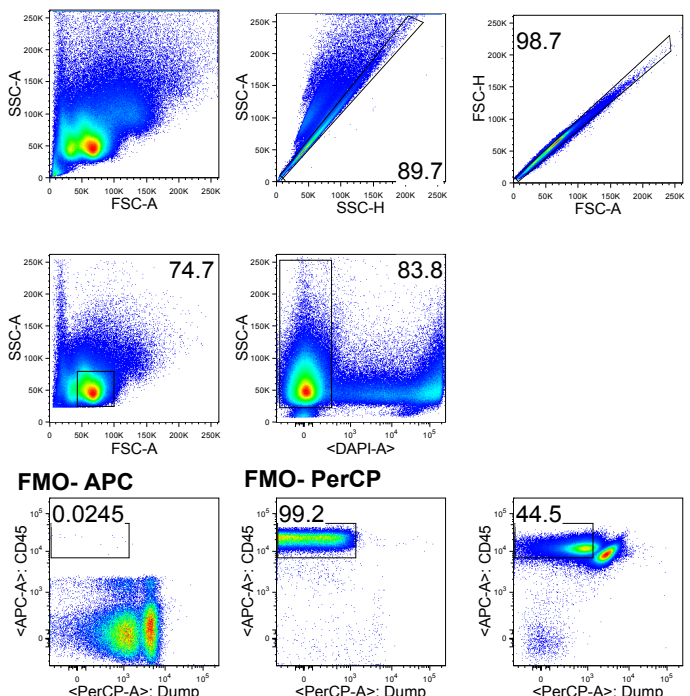

MO- PerCP

MO- Pacific Blue
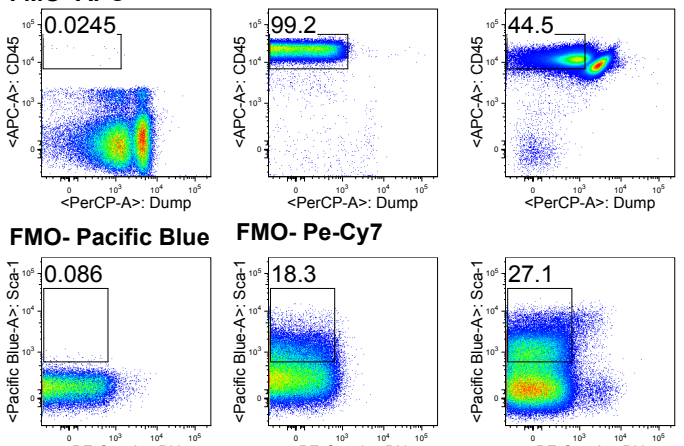

FMO- Pe-Cy7

MO- APC-Cy7

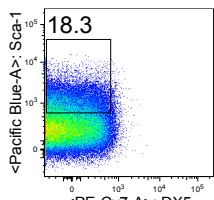

$\stackrel{8}{<P E-C y 7-A>: D X 5^{10^{3}}}$

$\sqrt[75.4]{70^{5}} \sqrt[5]{75.4}$

FMO- PE
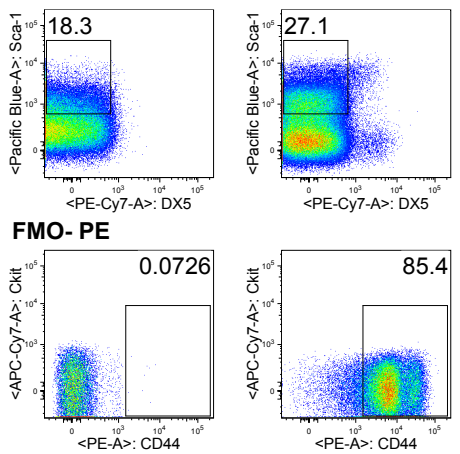

Figure A. 6 The FACS plots are read from left to right and represent the hierarchical gating strategy used for analysing nuocytes. (Doublet, Lymphocyte gate, Live (DAPI), FMO-APC gating controls for $\mathrm{CD} 5^{+}$, $\mathrm{FMO}$-PerCP gating controls for dump channel of Lineage cells (CD3, B220, FceR1a, CD4, CD8, CD11c, GR1), FMO-Pacific Blue gating controls for Ly6A/E $(\mathrm{Sca}-1)^{+}$, FMO-PE-Cy7 gating controls for CD49b (DX5)', FMO-APC-Cy7 gating controls for CD117 (Ckit) ${ }^{+}$, FMO-PE gating controls for $\mathrm{CD}_{4} 4^{+}$) 



References: 
1 Qian, D. \& Weiss, A. T cell antigen receptor signal transduction. Curr Opin Cell Biol 9, 205-212 (1997).

2 Sakaguchi, S., Sakaguchi, N., Asano, M., Itoh, M. \& Toda, M. Immunologic self-tolerance maintained by activated $\mathrm{T}$ cells expressing IL-2 receptor alphachains (CD25). Breakdown of a single mechanism of self-tolerance causes various autoimmune diseases. J Immunol 155, 1151-1164 (1995).

3 Chen, W., Jin, W., Hardegen, N., Lei, K.-J., Li, L., Marinos, N., McGrady, G. \& Wahl, S. M. Conversion of peripheral CD4+CD25- naive $\mathrm{T}$ cells to CD4+CD25+ regulatory $\mathrm{T}$ cells by TGF-beta induction of transcription factor Foxp3. J Exp Med 198, 1875-1886, doi:10.1084/jem.20030152 (2003).

Hsieh, C. S., Macatonia, S. E., Tripp, C. S., Wolf, S. F., O'Garra, A. \& Murphy, K. M. Development of TH1 CD4+ T cells through IL-12 produced by Listeria-induced macrophages. Science 260, 547-549 (1993).

$5 \quad$ Killar, L., MacDonald, G., West, J., Woods, A. \& Bottomly, K. Cloned, Iarestricted $\mathrm{T}$ cells that do not produce interleukin 4(IL 4)/B cell stimulatory factor 1(BSF-1) fail to help antigen-specific B cells. J Immunol 138, 16741679 (1987).

6 Mosmann, T. R., Cherwinski, H., Bond, M. W., Giedlin, M. A. \& Coffman, R. L. Two types of murine helper T cell clone. I. Definition according to profiles of lymphokine activities and secreted proteins. J Immunol 136, 2348-2357 (1986).

7 Swain, S. L., Weinberg, A. D., English, M. \& Huston, G. IL-4 directs the development of Th2-like helper effectors. J Immunol 145, 3796-3806 (1990).

8 Le Gros, G., Ben-Sasson, S. Z., Seder, R., Finkelman, F. D. \& Paul, W. E. Generation of interleukin 4 (IL-4)-producing cells in vivo and in vitro: IL-2 and IL-4 are required for in vitro generation of IL-4-producing cells. $J$ Exp Med 172, 921-929 (1990).

9 Veldhoen, M., Hocking, R. J., Atkins, C. J., Locksley, R. M. \& Stockinger, B. TGFbeta in the context of an inflammatory cytokine milieu supports de novo differentiation of IL-17-producing $\mathrm{T}$ cells. Immunity 24, 179-189, doi:10.1016/j.immuni.2006.01.001 (2006).

10 Mangan, P. R., Harrington, L. E., O'quinn, D. B., Helms, W. S., Bullard, D. C., Elson, C. O., Hatton, R. D., Wahl, S. M., Schoeb, T. R. \& Weaver, C. T. Transforming growth factor-beta induces development of the $\mathrm{T}(\mathrm{H}) 17$ lineage. Nature 441, 231-234, doi:10.1038/nature04754 (2006).

11 Bettelli, E., Carrier, Y., Gao, W., Korn, T., Strom, T. B., Oukka, M., Weiner, H. L. \& Kuchroo, V. K. Reciprocal developmental pathways for the generation of pathogenic effector TH17 and regulatory T cells. Nature 441, 235-238, doi:10.1038/nature04753 (2006).

12 Trifari, S., Kaplan, C. D., Tran, E. H., Crellin, N. K. \& Spits, H. Identification of a human helper $\mathrm{T}$ cell population that has abundant production of interleukin 22 and is distinct from $\mathrm{T}(\mathrm{H})-17, \mathrm{~T}(\mathrm{H}) 1$ and $\mathrm{T}(\mathrm{H}) 2$ cells. Nature Immunology 10, 864-871, doi:10.1038/ni.1770 (2009).

13 Duhen, T., Geiger, R., Jarrossay, D., Lanzavecchia, A. \& Sallusto, F. Production of interleukin 22 but not interleukin 17 by a subset of human skinhoming memory $\mathrm{T}$ cells. Nature Immunology 10, 857-863, doi:10.1038/ni.1767 (2009).

14 Veldhoen, M., Uyttenhove, C., van Snick, J., Helmby, H., Westendorf, A., Buer, J., Martin, B., Wilhelm, C. \& Stockinger, B. Transforming growth factor-beta 'reprograms' the differentiation of T helper 2 cells and promotes an 
interleukin 9-producing subset. Nat Immunol 9, 1341-1346, doi:10.1038/ni.1659 (2008).

15 Schmitt, E., Germann, T., Goedert, S., Hoehn, P., Huels, C., Koelsch, S., Kühn, R., Müller, W., Palm, N. \& Rüde, E. IL-9 production of naive CD4+ T cells depends on IL-2, is synergistically enhanced by a combination of TGFbeta and IL-4, and is inhibited by IFN-gamma. J Immunol 153, 3989-3996 (1994).

16 Dardalhon, V., Awasthi, A., Kwon, H., Galileos, G., Gao, W., Sobel, R. A., Mitsdoerffer, M., Strom, T. B., Elyaman, W., Ho, I.-C., Khoury, S., Oukka, M. \& Kuchroo, V. K. IL-4 inhibits TGF-beta-induced Foxp3+ T cells and, together with TGF-beta, generates IL-9+ IL-10+ Foxp3(-) effector T cells. Nature Immunology 9, 1347-1355, doi:10.1038/ni.1677 (2008).

17 Zhu, J. \& Paul, W. E. CD4 T cells: fates, functions, and faults. Blood 112, 1557-1569, doi:10.1182/blood-2008-05-078154 (2008).

18 Howard, M., Farrar, J., Hilfiker, M., Johnson, B., Takatsu, K., Hamaoka, T. \& Paul, W. E. Identification of a T cell-derived b cell growth factor distinct from interleukin 2. J Exp Med 155, 914-923 (1982).

19 Rabin, E. M., Ohara, J. \& Paul, W. E. B-cell stimulatory factor 1 activates resting B cells. Proc Natl Acad Sci USA 82, 2935-2939 (1985).

20 Cunningham, A. F., Serre, K., Toellner, K.-M., Khan, M., Alexander, J., Brombacher, F. \& MacLennan, I. C. M. Pinpointing IL-4-independent acquisition and IL-4-influenced maintenance of Th2 activity by CD4 T cells. Eur. J. Immunol. 34, 686-694, doi:10.1002/eji.200324510 (2004).

21 Noelle, R., Krammer, P. H., Ohara, J., Uhr, J. W. \& Vitetta, E. S. Increased expression of Ia antigens on resting B cells: an additional role for B-cell growth factor. Proc Natl Acad Sci USA 81, 6149-6153 (1984).

22 Lebman, D. A. \& Coffman, R. L. Interleukin 4 causes isotype switching to $\operatorname{IgE}$ in T cell-stimulated clonal B cell cultures. J Exp Med 168, 853-862 (1988).

23 Sherman, M. A., Secor, V. H. \& Brown, M. A. IL-4 preferentially activates a novel STAT6 isoform in mast cells. J Immunol 162, 2703-2708 (1999).

24 Xia, H. Z., Du, Z., Craig, S., Klisch, G., Noben-Trauth, N., Kochan, J. P., Huff, T. H., Irani, A. M. \& Schwartz, L. B. Effect of recombinant human IL-4 on tryptase, chymase, and Fc epsilon receptor type I expression in recombinant human stem cell factor-dependent fetal liver-derived human mast cells. $J$ Immunol 159, 2911-2921 (1997).

25 Toru, H., Ra, C., Nonoyama, S., Suzuki, K., Yata, J. \& Nakahata, T. Induction of the high-affinity IgE receptor (Fc epsilon RI) on human mast cells by IL-4. Int Immunol 8, 1367-1373 (1996).

26 Stein, M., Keshav, S., Harris, N. \& Gordon, S. Interleukin 4 potently enhances murine macrophage mannose receptor activity: a marker of alternative immunologic macrophage activation. J Exp Med 176, 287-292 (1992).

27 Chen, L., Grabowski, K. A., Xin, J.-P., Coleman, J., Huang, Z., Espiritu, B., Alkan, S., Xie, H. B., Zhu, Y., White, F. A., Clancy, J. \& Huang, H. IL-4 induces differentiation and expansion of Th2 cytokine-producing eosinophils. J Immunol 172, 2059-2066 (2004).

28 Zünd, G., Madara, J. L., Dzus, A. L., Awtrey, C. S. \& Colgan, S. P. Interleukin-4 and interleukin-13 differentially regulate epithelial chloride secretion. J Biol Chem 271, 7460-7464 (1996).

29 Shea-Donohue, T., Sullivan, C., Finkelman, F. D., Madden, K. B., Morris, S. C., Goldhill, J., Piñeiro-Carrero, V. \& Urban, J. F. The role of IL-4 in 
Heligmosomoides polygyrus-induced alterations in murine intestinal epithelial cell function. J Immunol 167, 2234-2239 (2001).

30 Madden, K. B., Whitman, L., Sullivan, C., Gause, W. C., Urban, J. F., Katona, I. M., Finkelman, F. D. \& Shea-Donohue, T. Role of STAT6 and mast cells in IL-4- and IL-13-induced alterations in murine intestinal epithelial cell function. J Immunol 169, 4417-4422 (2002).

31 Dabbagh, K., Takeyama, K., Lee, H. M., Ueki, I. F., Lausier, J. A. \& Nadel, J. A. IL-4 induces mucin gene expression and goblet cell metaplasia in vitro and in vivo. J Immunol 162, 6233-6237 (1999).

32 Ruedl, C., Bachmann, M. F. \& Kopf, M. The antigen dose determines T helper subset development by regulation of CD40 ligand. Eur. J. Immunol. 30, 20562064, doi:10.1002/1521-4141(200007)30:7\&\#60;2056::AIDIMMU2056\&\#62;3.0.CO;2-S (2000).

33 Constant, S., Pfeiffer, C., Woodard, A., Pasqualini, T. \& Bottomly, K. Extent of $\mathrm{T}$ cell receptor ligation can determine the functional differentiation of naive CD4+ T cells. J Exp Med 182, 1591-1596 (1995).

34 Boonstra, A., Asselin-Paturel, C., Gilliet, M., Crain, C., Trinchieri, G., Liu, Y.J. \& O'Garra, A. Flexibility of mouse classical and plasmacytoid-derived dendritic cells in directing $\mathrm{T}$ helper type 1 and 2 cell development: dependency on antigen dose and differential toll-like receptor ligation. J Exp Med 197, 101-109 (2003).

35 Pfeiffer, C., Stein, J., Southwood, S., Ketelaar, H., Sette, A. \& Bottomly, K. Altered peptide ligands can control CD4 T lymphocyte differentiation in vivo. J Exp Med 181, 1569-1574 (1995).

36 Esch, R. E. Allergens. Clin Rev Allergy Immunol 30, 71-72, doi:10.1385/CRIAI:30:2:71 (2006).

37 Steinman, R. M. Lasker Basic Medical Research Award. Dendritic cells: versatile controllers of the immune system. Nature Medicine 13, 1155-1159, doi:10.1038/nm1643 (2007).

38 Sokol, C. L., Chu, N.-Q., Yu, S., Nish, S. A., Laufer, T. M. \& Medzhitov, R. Basophils function as antigen-presenting cells for an allergen-induced T helper type 2 response. Nature Immunology 10, 713-720, doi:10.1038/ni.1738 (2009).

39 Perrigoue, J. G., Saenz, S. A., Siracusa, M. C., Allenspach, E. J., Taylor, B. C., Giacomin, P. R., Nair, M. G., Du, Y., Zaph, C., van Rooijen, N., Comeau, M. R., Pearce, E. J., Laufer, T. M. \& Artis, D. MHC class II-dependent basophilCD4+ $\mathrm{T}$ cell interactions promote $\mathrm{T}(\mathrm{H}) 2$ cytokine-dependent immunity. Nature Immunology 10, 697-705, doi:10.1038/ni.1740 (2009).

40 Yoshimoto, T., Yasuda, K., Tanaka, H., Nakahira, M., Imai, Y., Fujimori, Y. \& Nakanishi, K. Basophils contribute to $\mathrm{T}(\mathrm{H}) 2-\mathrm{IgE}$ responses in vivo via IL-4 production and presentation of peptide-MHC class II complexes to CD4+ T cells. Nature Immunology 10, 706-712, doi:10.1038/ni.1737 (2009).

41 Traidl-Hoffmann, C., Mariani, V., Hochrein, H., Karg, K., Wagner, H., Ring, J., Mueller, M. J., Jakob, T. \& Behrendt, H. Pollen-associated phytoprostanes inhibit dendritic cell interleukin-12 production and augment $\mathrm{T}$ helper type 2 cell polarization. $J$ Exp Med 201, 627-636, doi:10.1084/jem.20041065 (2005). van Rijt, L. S., Jung, S., Kleinjan, A., Vos, N., Willart, M., Duez, C., Hoogsteden, H. C. \& Lambrecht, B. N. In vivo depletion of lung CD11c+ dendritic cells during allergen challenge abrogates the characteristic features of asthma. J Exp Med 201, 981-991, doi:10.1084/jem.20042311 (2005). 
43 Kobayashi, T., Iijima, K., Radhakrishnan, S., Mehta, V., Vassallo, R., Lawrence, C. B., Cyong, J.-C., Pease, L. R., Oguchi, K. \& Kita, H. Asthmarelated environmental fungus, Alternaria, activates dendritic cells and produces potent Th2 adjuvant activity. J Immunol 182, 2502-2510, doi:10.4049/jimmunol.0802773 (2009).

44 Hammad, H., Plantinga, M., Deswarte, K., Pouliot, P., Willart, M. A. M., Kool, M., Muskens, F. \& Lambrecht, B. N. Inflammatory dendritic cells--not basophils--are necessary and sufficient for induction of Th2 immunity to inhaled house dust mite allergen. $J$ Exp Med 207, 2097-2111, doi:10.1084/jem.20101563 (2010).

45 Kim, S., Prout, M., Ramshaw, H., Lopez, A. F., Legros, G. \& Min, B. Cutting edge: basophils are transiently recruited into the draining lymph nodes during helminth infection via IL-3, but infection-induced Th2 immunity can develop without basophil lymph node recruitment or IL-3. J Immunol 184, 1143-1147, doi:10.4049/jimmunol.0902447 (2010).

46 Amsen, D., Blander, J. M., Lee, G. R., Tanigaki, K., Honjo, T. \& Flavell, R. A. Instruction of distinct CD4 T helper cell fates by different notch ligands on antigen-presenting cells. Cell 117, 515-526 (2004).

47 Jenkins, S. J., Perona-Wright, G., Worsley, A. G. F., Ishii, N. \& MacDonald, A. S. Dendritic cell expression of OX40 ligand acts as a costimulatory, not polarizing, signal for optimal Th2 priming and memory induction in vivo. $J$ Immunol 179, 3515-3523 (2007).

48 Nurieva, R. I., Duong, J., Kishikawa, H., Dianzani, U., Rojo, J. M., Ho, I. c., Flavell, R. A. \& Dong, C. Transcriptional regulation of th2 differentiation by inducible costimulator. Immunity 18, 801-811 (2003).

49 MacDonald, A. S., Straw, A. D., Dalton, N. M. \& Pearce, E. J. Cutting edge: Th2 response induction by dendritic cells: a role for CD40. J Immunol 168, 537-540 (2002).

50 Fowell, D. J., Magram, J., Turck, C. W., Killeen, N. \& Locksley, R. M. Impaired Th2 subset development in the absence of CD4. Immunity 6, 559-569 (1997).

51 Rulifson, I. C., Sperling, A. I., Fields, P. E., Fitch, F. W. \& Bluestone, J. A. CD28 costimulation promotes the production of Th2 cytokines. $J$ Immunol 158, 658-665 (1997).

52 Ierna, M. X., Scales, H. E., Saunders, K. L. \& Lawrence, C. E. Mast cell production of IL-4 and TNF may be required for protective and pathological responses in gastrointestinal helminth infection. Mucosal immunology 1, 147155, doi:10.1038/mi.2007.16 (2008).

53 Brown, M. A., Pierce, J. H., Watson, C. J., Falco, J., Ihle, J. N. \& Paul, W. E. $\mathrm{B}$ cell stimulatory factor-1/interleukin- 4 mRNA is expressed by normal and transformed mast cells. Cell 50, 809-818 (1987).

54 Min, B., Prout, M., Hu-Li, J., Zhu, J., Jankovic, D., Morgan, E. S., Urban, J. F., Dvorak, A. M., Finkelman, F. D., LeGros, G. \& Paul, W. E. Basophils produce IL-4 and accumulate in tissues after infection with a Th2-inducing parasite. J Exp Med 200, 507-517, doi:10.1084/jem.20040590 (2004).

55 van Panhuys, N., Prout, M., Forbes, E., Min, B., Paul, W. E. \& Le Gros, G. Basophils Are the Major Producers of IL-4 during Primary Helminth Infection. J Immunol, doi:10.4049/jimmunol.1000940 (2011). 
56 Yoshimoto, T. \& Paul, W. E. CD4pos, NK1.1pos T cells promptly produce interleukin 4 in response to in vivo challenge with anti-CD3. J Exp Med 179, 1285-1295 (1994).

57 Leite-De-Moraes, M. C., Hameg, A., Pacilio, M., Koezuka, Y., Taniguchi, M., Van Kaer, L., Schneider, E., Dy, M. \& Herbelin, A. IL-18 enhances IL-4 production by ligand-activated NKT lymphocytes: a pro-Th2 effect of IL-18 exerted through NKT cells. J Immunol 166, 945-951 (2001).

58 Akbari, O., Stock, P., Meyer, E., Kronenberg, M., Sidobre, S., Nakayama, T., Taniguchi, M., Grusby, M. J., DeKruyff, R. H. \& Umetsu, D. T. Essential role of NKT cells producing IL-4 and IL-13 in the development of allergeninduced airway hyperreactivity. Nature Medicine 9, 582-588, doi:10.1038/nm851 (2003).

59 Kitamura, H., Ohta, A., Sekimoto, M., Sato, M., Iwakabe, K., Nakui, M., Yahata, T., Meng, H., Koda, T., Nishimura, S., Kawano, T., Taniguchi, M. \& Nishimura, T. alpha-galactosylceramide induces early B-cell activation through IL-4 production by NKT cells. Cell Immunol 199, 37-42, doi:10.1006/cimm.1999.1602 (2000).

60 Johansson-Lindbom, B. \& Borrebaeck, C. A. K. Germinal center B cells constitute a predominant physiological source of IL-4: implication for Th2 development in vivo. J Immunol 168, 3165-3172 (2002).

61 Neill, D. R., Wong, S. H., Bellosi, A., Flynn, R. J., Daly, M., Langford, T. K. A., Bucks, C., Kane, C. M., Fallon, P. G., Pannell, R., Jolin, H. E. \& McKenzie, A. N. J. Nuocytes represent a new innate effector leukocyte that mediates type-2 immunity. Nature, doi:10.1038/nature08900 (2010).

62 Yagi, R., Suzuki, W., Seki, N., Kohyama, M., Inoue, T., Arai, T. \& Kubo, M. The IL-4 production capability of different strains of naive CD4(+) T cells controls the direction of the T(h) cell response. Int Immunol 14, 1-11 (2002).

63 Noben-Trauth, N., Hu-Li, J. \& Paul, W. E. Conventional, naive CD4+ T cells provide an initial source of IL-4 during Th2 differentiation. J Immunol 165, 3620-3625 (2000).

64 Jankovic, D., Kullberg, M. C., Noben-Trauth, N., Caspar, P., Paul, W. E. \& Sher, A. Single cell analysis reveals that IL-4 receptor/Stat6 signaling is not required for the in vivo or in vitro development of CD4+ lymphocytes with a Th2 cytokine profile. J Immunol 164, 3047-3055 (2000).

65 van Panhuys, N., Tang, S.-C., Prout, M., Camberis, M., Scarlett, D., Roberts, J., Hu-Li, J., Paul, W. E. \& Le Gros, G. In vivo studies fail to reveal a role for IL-4 or STAT6 signaling in Th2 lymphocyte differentiation. Proc Natl Acad Sci USA 105, 12423-12428, doi:10.1073/pnas.0806372105 (2008).

66 Yu, Q., Sharma, A., Oh, S. Y., Moon, H.-G., Hossain, M. Z., Salay, T. M., Leeds, K. E., Du, H., Wu, B., Waterman, M. L., Zhu, Z. \& Sen, J. M. T cell factor 1 initiates the $\mathrm{T}$ helper type 2 fate by inducing the transcription factor GATA-3 and repressing interferon-gamma. Nature Immunology 10, 992-999, doi:10.1038/ni.1762 (2009).

67 Cote-Sierra, J., Foucras, G., Guo, L., Chiodetti, L., Young, H. A., Hu-Li, J., Zhu, J. \& Paul, W. E. Interleukin 2 plays a central role in Th2 differentiation. Proc Natl Acad Sci USA 101, 3880-3885, doi:10.1073/pnas.0400339101 (2004).

68 Oliphant, C. J., Barlow, J. L. \& Mckenzie, A. N. J. Insights into the initiation of type 2 immune responses. Immunology 134, 378-385, doi:10.1111/j.13652567.2011.03499.x (2011). 
69 Omori-Miyake, M. \& Ziegler, S. F. Mouse models of allergic diseases: TSLP and its functional roles. Allergol Int 61, 27-34, doi:10.2332/allergolint.11-RAI0374 (2012).

70 Zhang, D. H., Cohn, L., Ray, P., Bottomly, K. \& Ray, A. Transcription factor GATA-3 is differentially expressed in murine Th1 and Th2 cells and controls Th2-specific expression of the interleukin-5 gene. J Biol Chem 272, 2159721603 (1997).

71 Zheng, W. \& Flavell, R. A. The transcription factor GATA-3 is necessary and sufficient for Th2 cytokine gene expression in CD4 T cells. Cell 89, 587-596 (1997).

72 Kurata, H., Lee, H. J., O'Garra, A. \& Arai, N. Ectopic expression of activated Stat6 induces the expression of Th2-specific cytokines and transcription factors in developing Th1 cells. Immunity 11, 677-688 (1999).

73 Zhu, J., Guo, L., Watson, C. J., Hu-Li, J. \& Paul, W. E. Stat6 is necessary and sufficient for IL-4's role in Th2 differentiation and cell expansion. $J$ Immunol 166, 7276-7281 (2001).

74 Kagami, S., Nakajima, H., Suto, A., Hirose, K., Suzuki, K., Morita, S., Kato, I., Saito, Y., Kitamura, T. \& Iwamoto, I. Stat5a regulates T helper cell differentiation by several distinct mechanisms. Blood 97, 2358-2365 (2001).

75 Zhu, J., Cote-Sierra, J., Guo, L. \& Paul, W. E. Stat5 activation plays a critical role in Th2 differentiation. Immunity 19, 739-748 (2003).

76 Yamane, H., Zhu, J. \& Paul, W. E. Independent roles for IL-2 and GATA-3 in stimulating naive CD4+ $\mathrm{T}$ cells to generate a Th2-inducing cytokine environment. J Exp Med 202, 793-804, doi:10.1084/jem.20051304 (2005).

77 Levin, S. D., Koelling, R. M., Friend, S. L., Isaksen, D. E., Ziegler, S. F., Perlmutter, R. M. \& Farr, A. G. Thymic stromal lymphopoietin: a cytokine that promotes the development of $\operatorname{IgM}+\mathrm{B}$ cells in vitro and signals via a novel mechanism. J Immunol 162, 677-683 (1999).

78 Isaksen, D. E., Baumann, H., Trobridge, P. A., Farr, A. G., Levin, S. D. \& Ziegler, S. F. Requirement for stat5 in thymic stromal lymphopoietin-mediated signal transduction. J Immunol 163, 5971-5977 (1999).

79 He, R., Oyoshi, M. K., Garibyan, L., Kumar, L., Ziegler, S. F. \& Geha, R. S. TSLP acts on infiltrating effector T cells to drive allergic skin inflammation. Proc Natl Acad Sci USA 105, 11875-11880, doi:10.1073/pnas.0801532105 (2008).

80 Guo, L., Wei, G., Zhu, J., Liao, W., Leonard, W. J., Zhao, K. \& Paul, W. IL-1 family members and STAT activators induce cytokine production by Th2, Th17, and Th1 cells. Proc Natl Acad Sci USA 106, 13463-13468, doi:10.1073/pnas.0906988106 (2009).

81 Angkasekwinai, P., Park, H., Wang, Y.-H., Wang, Y.-H., Chang, S. H., Corry, D. B., Liu, Y.-J., Zhu, Z. \& Dong, C. Interleukin 25 promotes the initiation of proallergic type 2 responses. J Exp Med 204, 1509-1517, doi:10.1084/jem.20061675 (2007).

82 Amsen, D., Antov, A., Jankovic, D., Sher, A., Radtke, F., Souabni, A., Busslinger, M., McCright, B., Gridley, T. \& Flavell, R. A. Direct regulation of Gata3 expression determines the $\mathrm{T}$ helper differentiation potential of Notch. Immunity 27, 89-99, doi:10.1016/j.immuni.2007.05.021 (2007).

83 Fang, T. C., Yashiro-Ohtani, Y., Del Bianco, C., Knoblock, D. M., Blacklow, S. C. \& Pear, W. S. Notch directly regulates Gata3 expression during T helper 
2 cell differentiation. Immunity $\mathbf{2 7}$ 100-110, doi:10.1016/j.immuni.2007.04.018 (2007).

84 Ho, I.-C., Tai, T.-S. \& Pai, S.-Y. GATA3 and the T-cell lineage: essential functions before and after T-helper-2-cell differentiation. Nat Rev Immunol 9, 125-135, doi:10.1038/nri2476 (2009).

85 Crotty, S., Johnston, R. J. \& Schoenberger, S. P. Effectors and memories: Bcl6 and Blimp-1 in T and B lymphocyte differentiation. Nature Immunology 11, 114-120, doi:10.1038/ni.1837 (2010).

86 Dent, A. L., Shaffer, A. L., Yu, X., Allman, D. \& Staudt, L. M. Control of inflammation, cytokine expression, and germinal center formation by BCL-6. Science 276, 589-592 (1997).

87 King, C., Tangye, S. G. \& Mackay, C. R. T follicular helper (TFH) cells in normal and dysregulated immune responses. Annu Rev Immunol 26, 741-766, doi:10.1146/annurev.immunol.26.021607.090344 (2008).

88 MacLennan, I. C. Germinal centers. Annu Rev Immunol 12, 117-139, doi:10.1146/annurev.iy.12.040194.001001 (1994).

89 Liu, Y. J., Malisan, F., de Bouteiller, O., Guret, C., Lebecque, S., Banchereau, J., Mills, F. C., Max, E. E. \& Martinez-Valdez, H. Within germinal centers, isotype switching of immunoglobulin genes occurs after the onset of somatic mutation. Immunity 4, 241-250 (1996).

90 Gatto, D. \& Brink, R. The germinal center reaction. J Allergy Clin Immunol 126, 898-907; quiz 908-899, doi:10.1016/j.jaci.2010.09.007 (2010).

91 Manz, R. A., Hauser, A. E., Hiepe, F. \& Radbruch, A. Maintenance of serum antibody levels. Annu Rev Immunol 23, 367-386, doi:10.1146/annurev.immunol.23.021704.115723 (2005).

92 MacLennan, I. C. M., Toellner, K.-M., Cunningham, A. F., Serre, K., Sze, D. M.-Y., Zúñiga, E., Cook, M. C. \& Vinuesa, C. G. Extrafollicular antibody responses. Immunol Rev 194, 8-18 (2003).

93 MacLennan, I. C. Somatic mutation. From the dark zone to the light. Curr Biol 4, 70-72 (1994).

94 Cyster, J. G., Ansel, K. M., Reif, K., Ekland, E. H., Hyman, P. L., Tang, H. L., Luther, S. A. \& Ngo, V. N. Follicular stromal cells and lymphocyte homing to follicles. Immunol Rev 176, 181-193 (2000).

95 Ansel, K. M., McHeyzer-Williams, L. J., Ngo, V. N., McHeyzer-Williams, M. G. \& Cyster, J. G. In vivo-activated CD4 T cells upregulate CXC chemokine receptor 5 and reprogram their response to lymphoid chemokines. $J$ Exp Med 190, 1123-1134 (1999).

96 Förster, R., Mattis, A. E., Kremmer, E., Wolf, E., Brem, G. \& Lipp, M. A putative chemokine receptor, BLR1, directs B cell migration to defined lymphoid organs and specific anatomic compartments of the spleen. Cell 87, 1037-1047 (1996).

97 Ansel, K. M., Ngo, V. N., Hyman, P. L., Luther, S. A., Förster, R., Sedgwick, J. D., Browning, J. L., Lipp, M. \& Cyster, J. G. A chemokine-driven positive feedback loop organizes lymphoid follicles. Nature 406, 309-314, doi:10.1038/35018581 (2000).

98 Reif, K., Ekland, E. H., Ohl, L., Nakano, H., Lipp, M., Förster, R. \& Cyster, J. G. Balanced responsiveness to chemoattractants from adjacent zones determines B-cell position. Nature 416, 94-99, doi:10.1038/416094a (2002).

99 Förster, R., Schubel, A., Breitfeld, D., Kremmer, E., Renner-Müller, I., Wolf, E. \& Lipp, M. CCR7 coordinates the primary immune response by 
establishing functional microenvironments in secondary lymphoid organs. Cell 99, 23-33 (1999).

100 Haynes, N. M., Allen, C. D. C., Lesley, R., Ansel, K. M., Killeen, N. \& Cyster, J. G. Role of CXCR5 and CCR7 in follicular Th cell positioning and appearance of a programmed cell death gene-1high germinal center-associated subpopulation. J Immunol 179, 5099-5108 (2007).

101 Nurieva, R. I., Chung, Y., Martinez, G. J., Yang, X. O., Tanaka, S., Matskevitch, T. D., Wang, Y.-H. \& Dong, C. Bcl6 mediates the development of $\mathrm{T}$ follicular helper cells. Science 325, 1001-1005, doi:10.1126/science.1176676 (2009).

102 Yu, D., Rao, S., Tsai, L. M., Lee, S. K., He, Y., Sutcliffe, E. L., Srivastava, M., Linterman, M., Zheng, L., Simpson, N., Ellyard, J. I., Parish, I. A., Ma, C. S., Li, Q.-J., Parish, C. R., Mackay, C. R. \& Vinuesa, C. G. The transcriptional repressor Bcl-6 directs $\mathrm{T}$ follicular helper cell lineage commitment. Immunity 31, 457-468, doi:10.1016/j.immuni.2009.07.002 (2009).

103 Johnston, R. J., Poholek, A. C., Ditoro, D., Yusuf, I., Eto, D., Barnett, B., Dent, A. L., Craft, J. \& Crotty, S. Bcl6 and Blimp-1 are reciprocal and antagonistic regulators of T follicular helper cell differentiation. Science 325, 1006-1010, doi:10.1126/science.1175870 (2009).

104 van Kooten, C. \& Banchereau, J. CD40-CD40 ligand. Journal of Leukocyte Biology 67, 2-17 (2000).

105 Breitfeld, D., Ohl, L., Kremmer, E., Ellwart, J., Sallusto, F., Lipp, M. \& Förster, R. Follicular B helper T cells express CXC chemokine receptor 5, localize to B cell follicles, and support immunoglobulin production. $J$ Exp Med 192, 1545-1552 (2000).

106 Schaerli, P., Willimann, K., Lang, A. B., Lipp, M., Loetscher, P. \& Moser, B. $\mathrm{CXC}$ chemokine receptor 5 expression defines follicular homing $\mathrm{T}$ cells with B cell helper function. J Exp Med 192, 1553-1562 (2000).

107 Campbell, D. J., Kim, C. H. \& Butcher, E. C. Separable effector T cell populations specialized for B cell help or tissue inflammation. Nature Immunology 2, 876-881, doi:10.1038/ni0901-876 (2001).

108 Kim, C. H., Rott, L. S., Clark-Lewis, I., Campbell, D. J., Wu, L. \& Butcher, E. C. Subspecialization of CXCR5+ T cells: B helper activity is focused in a germinal center-localized subset of CXCR5+ T cells. J Exp Med 193, 13731381 (2001).

109 Zaretsky, A. G., Taylor, J. J., King, I. L., Marshall, F. A., Mohrs, M. \& Pearce, E. J. T follicular helper cells differentiate from Th2 cells in response to helminth antigens. Journal of Experimental Medicine 206, 991-999, doi:10.1084/jem.20090303 (2009).

110 King, I. L. \& Mohrs, M. IL-4-producing CD4+ T cells in reactive lymph nodes during helminth infection are T follicular helper cells. Journal of Experimental Medicine 206, 1001-1007, doi:10.1084/jem.20090313 (2009).

111 Reinhardt, R. L., Liang, H.-E. \& Locksley, R. M. Cytokine-secreting follicular $\mathrm{T}$ cells shape the antibody repertoire. Nature Immunology 10, 385-393, doi:10.1038/ni.1715 (2009).

112 Abbas, A. K., Urioste, S., Collins, T. L. \& Boom, W. H. Heterogeneity of helper/inducer T lymphocytes. IV. Stimulation of resting and activated B cells by Th1 and Th2 clones. J Immunol 144, 2031-2037 (1990).

113 Chtanova, T., Tangye, S. G., Newton, R., Frank, N., Hodge, M. R., Rolph, M. S. \& Mackay, C. R. T follicular helper cells express a distinctive 
transcriptional profile, reflecting their role as non-Th1/Th2 effector cells that provide help for B cells. J Immunol 173, 68-78 (2004).

114 Kim, C. H., Lim, H. W., Kim, J. R., Rott, L., Hillsamer, P. \& Butcher, E. C. Unique gene expression program of human germinal center $\mathrm{T}$ helper cells. Blood 104, 1952-1960, doi:10.1182/blood-2004-03-1206 (2004).

115 Gunn, M. D., Ngo, V. N., Ansel, K. M., Ekland, E. H., Cyster, J. G. \& Williams, L. T. A B-cell-homing chemokine made in lymphoid follicles activates Burkitt's lymphoma receptor-1. Nature 391, 799-803, doi:10.1038/35876 (1998).

116 Allen, C. D. C., Ansel, K. M., Low, C., Lesley, R., Tamamura, H., Fujii, N. \& Cyster, J. G. Germinal center dark and light zone organization is mediated by CXCR4 and CXCR5. Nature Immunology 5, 943-952, doi:10.1038/ni1100 (2004).

117 Yusuf, I., Kageyama, R., Monticelli, L., Johnston, R. J., Ditoro, D., Hansen, K., Barnett, B. \& Crotty, S. Germinal center T follicular helper cell IL-4 production is dependent on signaling lymphocytic activation molecule receptor (CD150). J Immunol 185, 190-202, doi:10.4049/jimmunol.0903505 (2010).

118 Vijayanand, P., Seumois, G., Simpson, L. J., Abdul-Wajid, S., Baumjohann, D., Panduro, M., Huang, X., Interlandi, J., Djuretic, I. M., Brown, D. R., Sharpe, A. H., Rao, A. \& Ansel, K. M. Interleukin-4 Production by Follicular Helper T Cells Requires the Conserved Il4 Enhancer Hypersensitivity Site V. Immunity, doi:10.1016/j.immuni.2011.12.014 (2012).

119 Hu-Li, J., Pannetier, C., Guo, L., Löhning, M., Gu, H., Watson, C., Assenmacher, M., Radbruch, A. \& Paul, W. E. Regulation of expression of IL4 alleles: analysis using a chimeric GFP/IL-4 gene. Immunity 14, 1-11 (2001).

120 Bix, M. \& Locksley, R. M. Independent and epigenetic regulation of the interleukin-4 alleles in CD4+ T cells. Science 281, 1352-1354 (1998).

121 Rivière, I., Sunshine, M. J. \& Littman, D. R. Regulation of IL-4 expression by activation of individual alleles. Immunity 9, 217-228 (1998).

122 Kracker, S. \& Durandy, A. Insights into the B cell specific process of immunoglobulin class switch recombination. Immunology Letters 138, 97-103, doi:10.1016/j.imlet.2011.02.004 (2011).

123 Tada, T., Okumura, K., Platteau, B., Beckers, A. \& Bazin, H. Half-lives of two types of rat homocytotropic antibodies in circulation and in the skin. Int Arch Allergy Appl Immunol 48, 116-131 (1975).

124 Pochanke, V., Koller, S., Dayer, R., Hatak, S., Ludewig, B., Zinkernagel, R. M., Hengartner, H. \& McCoy, K. D. Identification and characterization of a novel antigen from the nematode Nippostrongylus brasiliensis recognized by specific IgE. Eur. J. Immunol. 37, 1275-1284, doi:10.1002/eji.200737135 (2007).

125 MacGlashan, D. IgE receptor and signal transduction in mast cells and basophils. Curr Opin Immunol 20, 717-723, doi:10.1016/j.coi.2008.08.004 (2008).

126 Xiong, H., Dolpady, J., Wabl, M., Curotto de Lafaille, M. A. \& Lafaille, J. J. Sequential class switching is required for the generation of high affinity $\operatorname{IgE}$ antibodies. J Exp Med 209, 353-364, doi:10.1084/jem.20111941 (2012).

127 Yoshida, K., Matsuoka, M., Usuda, S., Mori, A., Ishizaka, K. \& Sakano, H. Immunoglobulin switch circular DNA in the mouse infected with Nippostrongylus brasiliensis: evidence for successive class switching from mu to epsilon via gamma 1. Proc Natl Acad Sci USA 87, 7829-7833 (1990). 
128 Talay, O., Yan, D., Brightbill, H. D., Straney, E. E. M., Zhou, M., Ladi, E., Lee, W. P., Egen, J. G., Austin, C. D., Xu, M. \& Wu, L. C. IgE(+) memory B cells and plasma cells generated through a germinal-center pathway. Nature Immunology 13, 396-404, doi:10.1038/ni.2256 (2012).

129 Yang, Z., Sullivan, B. M. \& Allen, C. D. C. Fluorescent In Vivo Detection Reveals that $\operatorname{IgE}(+)$ B Cells Are Restrained by an Intrinsic Cell Fate Predisposition. Immunity, doi:10.1016/j.immuni.2012.02.009 (2012).

130 Kühn, R., Rajewsky, K. \& Müller, W. Generation and analysis of interleukin-4 deficient mice. Science 254, 707-710 (1991).

131 Takeda, K., Tanaka, T., Shi, W., Matsumoto, M., Minami, M., Kashiwamura, S., Nakanishi, K., Yoshida, N., Kishimoto, T. \& Akira, S. Essential role of Stat6 in IL-4 signalling. Nature 380, 627-630, doi:10.1038/380627a0 (1996).

132 Finkelman, F. D., Katona, I. M., Urban, J. F., Holmes, J., Ohara, J., Tung, A. S., Sample, J. V. \& Paul, W. E. IL-4 is required to generate and sustain in vivo IgE responses. J Immunol 141, 2335-2341 (1988).

133 Ozaki, K., Spolski, R., Feng, C. G., Qi, C.-F., Cheng, J., Sher, A., Morse, H. C., Liu, C., Schwartzberg, P. L. \& Leonard, W. J. A critical role for IL-21 in regulating immunoglobulin production. Science (New York, NY) 298, 16301634, doi:10.1126/science.1077002 (2002).

134 Mohrs, M., Shinkai, K., Mohrs, K. \& Locksley, R. M. Analysis of type 2 immunity in vivo with a bicistronic IL-4 reporter. Immunity 15, 303-311 (2001).

135 Mariani, L., Schulz, E. G., Lexberg, M. H., Helmstetter, C., Radbruch, A., Löhning, M. \& Höfer, T. Short-term memory in gene induction reveals the regulatory principle behind stochastic IL-4 expression. Molecular Systems Biology 6, 359, doi:10.1038/msb.2010.13 (2010).

136 Hurst, S. D., Muchamuel, T., Gorman, D. M., Gilbert, J. M., Clifford, T., Kwan, S., Menon, S., Seymour, B., Jackson, C., Kung, T. T., Brieland, J. K., Zurawski, S. M., Chapman, R. W., Zurawski, G. \& Coffman, R. L. New IL-17 family members promote Th1 or Th2 responses in the lung: in vivo function of the novel cytokine IL-25. J Immunol 169, 443-453 (2002).

137 Pan, G., French, D., Mao, W., Maruoka, M., Risser, P., Lee, J., Foster, J., Aggarwal, S., Nicholes, K., Guillet, S., Schow, P. \& Gurney, A. L. Forced expression of murine IL-17E induces growth retardation, jaundice, a Th2biased response, and multiorgan inflammation in mice. J Immunol 167, 65596567 (2001).

138 Fort, M. M., Cheung, J., Yen, D., Li, J., Zurawski, S. M., Lo, S., Menon, S., Clifford, T., Hunte, B., Lesley, R., Muchamuel, T., Hurst, S. D., Zurawski, G., Leach, M. W., Gorman, D. M. \& Rennick, D. M. IL-25 induces IL-4, IL-5, and IL-13 and Th2-associated pathologies in vivo. Immunity 15, 985-995 (2001).

139 Lee, J., Ho, W. H., Maruoka, M., Corpuz, R. T., Baldwin, D. T., Foster, J. S., Goddard, A. D., Yansura, D. G., Vandlen, R. L., Wood, W. I. \& Gurney, A. L. IL-17E, a novel proinflammatory ligand for the IL-17 receptor homolog IL17Rh1. J Biol Chem 276, 1660-1664, doi:10.1074/jbc.M008289200 (2001).

140 Shi, Y., Ullrich, S. J., Zhang, J., Connolly, K., Grzegorzewski, K. J., Barber, M. C., Wang, W., Wathen, K., Hodge, V., Fisher, C. L., Olsen, H., Ruben, S. M., Knyazev, I., Cho, Y. H., Kao, V., Wilkinson, K. A., Carrell, J. A. \& Ebner, R. A novel cytokine receptor-ligand pair. Identification, molecular 
characterization, and in vivo immunomodulatory activity. J Biol Chem 275, 19167-19176, doi:10.1074/jbc.M910228199 (2000).

141 Tian, E., Sawyer, J. R., Largaespada, D. A., Jenkins, N. A., Copeland, N. G. \& Shaughnessy, J. D. Evi27 encodes a novel membrane protein with homology to the IL17 receptor. Oncogene 19, 2098-2109, doi:10.1038/sj.onc.1203577 (2000).

142 Maezawa, Y., Nakajima, H., Suzuki, K., Tamachi, T., Ikeda, K., Inoue, J.-i., Saito, Y. \& Iwamoto, I. Involvement of TNF receptor-associated factor 6 in IL-25 receptor signaling. J Immunol 176, 1013-1018 (2006).

143 Ikeda, K., Nakajima, H., Suzuki, K., Kagami, S.-I., Hirose, K., Suto, A., Saito, Y. \& Iwamoto, I. Mast cells produce interleukin-25 upon Fc epsilon RImediated activation. Blood 101, 3594-3596, doi:10.1182/blood-2002-09-2817 (2003).

144 Kang, C.-M., Jang, A.-S., Ahn, M.-H., Shin, J.-A., Kim, J.-H., Choi, Y.-S., Rhim, T.-Y. \& Park, C.-S. Interleukin-25 and interleukin-13 production by alveolar macrophages in response to particles. Am J Respir Cell Mol Biol 33, 290-296, doi:10.1165/rcmb.2005-0003OC (2005).

145 Owyang, A. M., Zaph, C., Wilson, E. H., Guild, K. J., McClanahan, T., Miller, H. R. P., Cua, D. J., Goldschmidt, M., Hunter, C. A., Kastelein, R. A. \& Artis, D. Interleukin 25 regulates type 2 cytokine-dependent immunity and limits chronic inflammation in the gastrointestinal tract. J Exp Med 203, 843-849, doi:10.1084/jem.20051496 (2006).

146 Gratchev, A., Kzhyshkowska, J., Duperrier, K., Utikal, J., Velten, F. W. \& Goerdt, S. The receptor for interleukin-17E is induced by Th2 cytokines in antigen-presenting cells. Scand J Immunol 60, 233-237, doi:10.1111/j.03009475.2004.01443.x (2004).

147 Wang, Y.-H., Angkasekwinai, P., Lu, N., Voo, K. S., Arima, K., Hanabuchi, S., Hippe, A., Corrigan, C. J., Dong, C., Homey, B., Yao, Z., Ying, S., Huston, D. P. \& Liu, Y.-J. IL-25 augments type 2 immune responses by enhancing the expansion and functions of TSLP-DC-activated Th2 memory cells. J Exp Med 204, 1837-1847, doi:10.1084/jem.20070406 (2007).

148 Terashima, A., Watarai, H., Inoue, S., Sekine, E., Nakagawa, R., Hase, K., Iwamura, C., Nakajima, H., Nakayama, T. \& Taniguchi, M. A novel subset of mouse NKT cells bearing the IL-17 receptor B responds to IL-25 and contributes to airway hyperreactivity. $J$ Exp Med 205, 2727-2733, doi:10.1084/jem.20080698 (2008).

149 Stock, P., Lombardi, V., Kohlrautz, V. \& Akbari, O. Induction of airway hyperreactivity by IL-25 is dependent on a subset of invariant NKT cells expressing IL-17RB. J Immunol 182, 5116-5122, doi:10.4049/jimmunol.0804213 (2009).

150 Price, A. E., Liang, H.-E., Sullivan, B. M., Reinhardt, R. L., Eisley, C. J., Erle, D. J. \& Locksley, R. M. Systemically dispersed innate IL-13-expressing cells in type 2 immunity. Proc Natl Acad Sci USA 107, 11489-11494, doi:10.1073/pnas.1003988107 (2010).

151 Saenz, S. A., Siracusa, M. C., Perrigoue, J. G., Spencer, S. P., Urban Jr, J. F., Tocker, J. E., Budelsky, A. L., Kleinschek, M. A., Kastelein, R. A., Kambayashi, T., Bhandoola, A. \& Artis, D. IL25 elicits a multipotent progenitor cell population that promotes $\mathrm{T}(\mathrm{H}) 2$ cytokine responses. Nature, doi:10.1038/nature08901 (2010). 
152 Moro, K., Yamada, T., Tanabe, M., Takeuchi, T., Ikawa, T., Kawamoto, H., Furusawa, J.-i., Ohtani, M., Fujii, H. \& Koyasu, S. Innate production of T(H)2 cytokines by adipose tissue-associated c-Kit(+)Sca-1(+) lymphoid cells. Nature, doi:10.1038/nature08636 (2009).

153 Urban, J. F., Katona, I. M. \& Finkelman, F. D. Heligmosomoides polygyrus: CD4+ but not CD8+ T cells regulate the IgE response and protective immunity in mice. EXPERIMENTAL PARASITOLOGY 73, 500-511 (1991).

154 Grencis, R. K., Riedlinger, J. \& Wakelin, D. L3T4-positive T lymphoblasts are responsible for transfer of immunity to Trichinella spiralis in mice. Immunology 56, 213-218 (1985).

155 Katona, I. M., Urban, J. F. \& Finkelman, F. D. The role of L3T4+ and Lyt-2+ $\mathrm{T}$ cells in the IgE response and immunity to Nippostrongylus brasiliensis. $J$ Immunol 140, 3206-3211 (1988).

156 Hotez, P. J., Brooker, S., Bethony, J. M., Bottazzi, M. E., Loukas, A. \& Xiao, S. Hookworm Infection. New England Journal of Medicine 351, 799-807, doi:doi:10.1056/NEJMra032492 (2004).

157 Siracusa, M. C., Reece, J. J., Urban, J. F. \& Scott, A. L. Dynamics of lung macrophage activation in response to helminth infection. Journal of Leukocyte Biology 84, 1422-1433, doi:10.1189/jlb.0308199 (2008).

158 Reece, J. J., Siracusa, M. C., Southard, T. L., Brayton, C. F., Urban, J. F. \& Scott, A. L. Hookworm-induced persistent changes to the immunological environment of the lung. Infect Immun 76, 3511-3524, doi:10.1128/IAI.0019208 (2008).

159 Mearns, H., Horsnell, W. G. C., Hoving, J. C., Dewals, B., Cutler, A. J., Kirstein, F., Myburgh, E., Arendse, B. \& Brombacher, F. Interleukin-4promoted $\mathrm{T}$ helper 2 responses enhance Nippostrongylus brasiliensis-induced pulmonary pathology. Infect Immun 76, 5535-5542, doi:10.1128/IAI.00210-08 (2008).

160 Marsland, B. J., Kurrer, M., Reissmann, R., Harris, N. L. \& Kopf, M. Nippostrongylus brasiliensis infection leads to the development of emphysema associated with the induction of alternatively activated macrophages. Eur. J. Immunol. 38, 479-488, doi:10.1002/eji.200737827 (2008).

161 Harvie, M., Camberis, M., Tang, S.-C., Delahunt, B., Paul, W. \& Le Gros, G. The lung is an important site for priming CD4 T-cell-mediated protective immunity against gastrointestinal helminth parasites. Infection and Immunity 78, 3753-3762, doi:10.1128/IAI.00502-09 (2010).

162 Camberis, M., Le Gros, G. \& Urban, J. Animal model of Nippostrongylus brasiliensis and Heligmosomoides polygyrus. Curr Protoc Immunol Chapter 19, Unit 19.12, doi:10.1002/0471142735.im1912s55 (2003).

163 Urban, J. F., Noben-Trauth, N., Donaldson, D. D., Madden, K. B., Morris, S. C., Collins, M. \& Finkelman, F. D. IL-13, IL-4Ralpha, and Stat6 are required for the expulsion of the gastrointestinal nematode parasite Nippostrongylus brasiliensis. Immunity 8, 255-264 (1998).

164 Finkelman, F. D., Shea-Donohue, T., Morris, S. C., Gildea, L., Strait, R., Madden, K. B., Schopf, L. \& Urban, J. F. Interleukin-4- and interleukin-13mediated host protection against intestinal nematode parasites. Immunol Rev 201, 139-155, doi:10.1111/j.0105-2896.2004.00192.x (2004).

165 Voehringer, D., Reese, T. A., Huang, X., Shinkai, K. \& Locksley, R. M. Type 2 immunity is controlled by IL-4/IL-13 expression in hematopoietic non- 
eosinophil cells of the innate immune system. J Exp Med 203, 1435-1446, doi:10.1084/jem.20052448 (2006).

166 Urban, J. F., Noben-Trauth, N., Schopf, L., Madden, K. B. \& Finkelman, F. D. Cutting edge: IL-4 receptor expression by non-bone marrow-derived cells is required to expel gastrointestinal nematode parasites. J Immunol 167, 60786081 (2001).

167 Fallon, P. G., Jolin, H. E., Smith, P., Emson, C. L., Townsend, M. J., Fallon, R., Smith, P. \& Mckenzie, A. N. J. IL-4 induces characteristic Th2 responses even in the combined absence of IL-5, IL-9, and IL-13. Immunity 17, 7-17 (2002).

168 McKenzie, G. J., Bancroft, A., Grencis, R. K. \& McKenzie, A. N. A distinct role for interleukin-13 in Th2-cell-mediated immune responses. Curr Biol 8, 339-342 (1998).

169 Finkelman, F. D., Shea-Donohue, T., Goldhill, J., Sullivan, C. A., Morris, S. C., Madden, K. B., Gause, W. C. \& Urban, J. F. Cytokine regulation of host defense against parasitic gastrointestinal nematodes: lessons from studies with rodent models. Annu Rev Immunol 15, 505-533, doi:10.1146/annurev.immunol.15.1.505 (1997).

170 Hasnain, S. Z., Evans, C. M., Roy, M., Gallagher, A. L., Kindrachuk, K. N., Barron, L., Dickey, B. F., Wilson, M. S., Wynn, T. A., Grencis, R. K. \& Thornton, D. J. Muc5ac: a critical component mediating the rejection of enteric nematodes. Journal of Experimental Medicine, doi:10.1084/jem.20102057 (2011).

171 Artis, D., Wang, M. L., Keilbaugh, S. A., He, W., Brenes, M., Swain, G. P., Knight, P. A., Donaldson, D. D., Lazar, M. A., Miller, H. R. P., Schad, G. A., Scott, P. \& Wu, G. D. RELMbeta/FIZZ2 is a goblet cell-specific immuneeffector molecule in the gastrointestinal tract. Proc Natl Acad Sci USA 101, 13596-13600, doi:10.1073/pnas.0404034101 (2004).

172 Herbert, D. b. R., Yang, J.-Q., Hogan, S. P., Groschwitz, K., Khodoun, M., Munitz, A., Orekov, T., Perkins, C., Wang, Q., Brombacher, F., Urban, J. F., Rothenberg, M. E. \& Finkelman, F. D. Intestinal epithelial cell secretion of RELM-beta protects against gastrointestinal worm infection. J Exp Med 206, 2947-2957, doi:10.1084/jem.20091268 (2009).

173 Zhao, A., Urban, J. F., Anthony, R. M., Sun, R., Stiltz, J., Van Rooijen, N., Wynn, T. A., Gause, W. C. \& Shea-Donohue, T. Th2 cytokine-induced alterations in intestinal smooth muscle function depend on alternatively activated macrophages. Gastroenterology 135, 217-225.e211, doi:10.1053/j.gastro.2008.03.077 (2008).

174 Horsnell, W. G. C., Cutler, A. J., Hoving, J. C., Hoving, C. J., Mearns, H., Myburgh, E., Arendse, B., Finkelman, F. D., Owens, G. K., Erle, D. \& Brombacher, F. Delayed goblet cell hyperplasia, acetylcholine receptor expression, and worm expulsion in SMC-specific IL-4Ralpha-deficient mice. PLoS Pathog 3, e1, doi:10.1371/journal.ppat.0030001 (2007).

175 Fallon, P. G., Ballantyne, S. J., Mangan, N. E., Barlow, J. L., Dasvarma, A., Hewett, D. R., McIlgorm, A., Jolin, H. E. \& McKenzie, A. N. J. Identification of an interleukin (IL)-25-dependent cell population that provides IL-4, IL-5, and IL-13 at the onset of helminth expulsion. J Exp Med 203, 1105-1116, doi:10.1084/jem.20051615 (2006). 
176 Spits, H. \& Di Santo, J. P. The expanding family of innate lymphoid cells: regulators and effectors of immunity and tissue remodeling. Nature Immunology 12, 21-27, doi:10.1038/ni.1962 (2011).

177 Wong, S. H., Walker, J. A., Jolin, H. E., Drynan, L. F., Hams, E., Camelo, A., Barlow, J. L., Neill, D. R., Panova, V., Koch, U., Radtke, F., Hardman, C. S., Hwang, Y. Y., Fallon, P. G. \& Mckenzie, A. N. J. Transcription factor ROR $\alpha$ is critical for nuocyte development. Nature Immunology, doi:10.1038/ni.2208 (2012).

178 Koyasu, S. \& Moro, K. Type 2 innate immune responses and the natural helper cell. Immunology, doi:10.1111/j.1365-2567.2011.03413.x (2011).

179 Allen, J. E. \& Maizels, R. M. Diversity and dialogue in immunity to helminths. Nat Rev Immunol 11, 375-388, doi:10.1038/nri2992 (2011).

180 Bansemir, A. D. \& Sukhdeo, M. V. The food resource of adult Heligmosomoides polygyrus in the small intestine. J Parasitol 80, 24-28 (1994).

181 Maizels, R. M., Hewitson, J. P., Murray, J., Harcus, Y. M., Dayer, B., Filbey, K. J., Grainger, J. R., McSorley, H. J., Reynolds, L. A. \& Smith, K. A. Immune modulation and modulators in Heligmosomoides polygyrus infection. EXPERIMENTAL PARASITOLOGY, doi:10.1016/j.exppara.2011.08.011 (2011).

182 Urban, J. F., Katona, I. M., Paul, W. E. \& Finkelman, F. D. Interleukin 4 is important in protective immunity to a gastrointestinal nematode infection in mice. Proc Natl Acad Sci USA 88, 5513-5517 (1991).

183 Anthony, R. M., Urban, J. F., Alem, F., Hamed, H. A., Rozo, C. T., Boucher, J.-L., van Rooijen, N. \& Gause, W. C. Memory $\mathrm{T}(\mathrm{H}) 2$ cells induce alternatively activated macrophages to mediate protection against nematode parasites. Nat Med 12, 955-960, doi:10.1038/nm1451 (2006).

184 Wojciechowski, W., Harris, D. P., Sprague, F., Mousseau, B., Makris, M., Kusser, K., Honjo, T., Mohrs, K., Mohrs, M., Randall, T. \& Lund, F. E. Cytokine-producing effector B cells regulate type 2 immunity to $\mathrm{H}$. polygyrus. Immunity 30, 421-433, doi:10.1016/j.immuni.2009.01.006 (2009).

185 McCoy, K. D., Stoel, M., Stettler, R., Merky, P., Fink, K., Senn, B. M., Schaer, C., Massacand, J., Odermatt, B., Oettgen, H. C., Zinkernagel, R. M., Bos, N. A., Hengartner, H., Macpherson, A. J. \& Harris, N. L. Polyclonal and specific antibodies mediate protective immunity against enteric helminth infection. Cell Host Microbe 4, 362-373, doi:10.1016/j.chom.2008.08.014 (2008).

186 Morimoto, M., Morimoto, M., Whitmire, J., Xiao, S., Anthony, R. M., Mirakami, H., Star, R. A., Urban, J. F. \& Gause, W. C. Peripheral CD4 T cells rapidly accumulate at the host: parasite interface during an inflammatory Th2 memory response. J Immunol 172, 2424-2430 (2004).

187 Zhao, A., McDermott, J., Urban, J. F., Gause, W., Madden, K. B., Yeung, K. A., Morris, S. C., Finkelman, F. D. \& Shea-Donohue, T. Dependence of IL-4, IL-13, and nematode-induced alterations in murine small intestinal smooth muscle contractility on Stat6 and enteric nerves. J Immunol 171, 948-954 (2003).

188 Anthony, R. M., Rutitzky, L. I., Urban, J. F., Stadecker, M. J. \& Gause, W. C. Protective immune mechanisms in helminth infection. Nat Rev Immunol 7, 975-987, doi:10.1038/nri2199 (2007). 
189 Ho, I. C., Kaplan, M. H., Jackson-Grusby, L., Glimcher, L. H. \& Grusby, M. J. Marking IL-4-producing cells by knock-in of the IL-4 gene. Int Immunol 11, 243-247 (1999).

190 Mohrs, K., Wakil, A. E., Killeen, N., Locksley, R. M. \& Mohrs, M. A twostep process for cytokine production revealed by IL-4 dual-reporter mice. Immunity 23, 419-429, doi:10.1016/j.immuni.2005.09.006 (2005).

191 Sathe, P., Pooley, J., Vremec, D., Mintern, J., Jin, J.-O., Wu, L., Kwak, J.-Y., Villadangos, J. A. \& Shortman, K. The acquisition of antigen crosspresentation function by newly formed dendritic cells. J Immunol 186, 51845192, doi:10.4049/jimmunol.1002683 (2011).

192 Kaplan, M. H., Schindler, U., Smiley, S. T. \& Grusby, M. J. Stat6 is required for mediating responses to IL-4 and for development of Th2 cells. Immunity 4, 313-319 (1996).

193 Okabe, M., Ikawa, M., Kominami, K., Nakanishi, T. \& Nishimune, Y. 'Green mice' as a source of ubiquitous green cells. FEBS Lett 407, 313-319 (1997).

194 Kleinschek, M. A., Owyang, A. M., Joyce-Shaikh, B., Langrish, C. L., Chen, Y., Gorman, D. M., Blumenschein, W. M., McClanahan, T., Brombacher, F., Hurst, S. D., Kastelein, R. A. \& Cua, D. J. IL-25 regulates Th17 function in autoimmune inflammation. $J$ Exp Med 204, 161-170, doi:10.1084/jem.20061738 (2007).

195 Barnden, M. J., Allison, J., Heath, W. R. \& Carbone, F. R. Defective TCR expression in transgenic mice constructed using cDNA-based alpha- and betachain genes under the control of heterologous regulatory elements. Immunol Cell Biol 76, 34-40, doi:10.1046/j.1440-1711.1998.00709.x (1998).

196 Kim, M. R., Manoukian, R., Yeh, R., Silbiger, S. M., Danilenko, D. M., Scully, S., Sun, J., DeRose, M. L., Stolina, M., Chang, D., Van, G. Y., Clarkin, K., Nguyen, H. Q., Yu, Y. B., Jing, S., Senaldi, G., Elliott, G. \& Medlock, E. S. Transgenic overexpression of human IL-17E results in eosinophilia, Blymphocyte hyperplasia, and altered antibody production. Blood 100, 23302340, doi:10.1182/blood-2002-01-0012 (2002).

197 Sharkhuu, T., Matthaei, K. I., Forbes, E., Mahalingam, S., Hogan, S. P., Hansbro, P. M. \& Foster, P. S. Mechanism of interleukin-25 (IL-17E)-induced pulmonary inflammation and airways hyper-reactivity. Clin Exp Allergy 36, 1575-1583, doi:10.1111/j.1365-2222.2006.02595.x (2006).

198 Tao, X., Grant, C., Constant, S. \& Bottomly, K. Induction of IL-4-producing CD4+ T cells by antigenic peptides altered for TCR binding. J Immunol 158, 4237-4244 (1997).

199 Hosken, N. A., Shibuya, K., Heath, A. W., Murphy, K. M. \& O'Garra, A. The effect of antigen dose on CD4+ T helper cell phenotype development in a T cell receptor-alpha beta-transgenic model. J Exp Med 182, 1579-1584 (1995).

200 Berzins, S. P., McNab, F. W., Jones, C. M., Smyth, M. J. \& Godfrey, D. I. Long-term retention of mature NK1.1+ NKT cells in the thymus. $J$ Immunol 176, 4059-4065 (2006).

201 Seidl, A., Panzer, M. \& Voehringer, D. Protective immunity against the gastrointestinal nematode Nippostrongylus brasiliensis requires a broad T-cell receptor repertoire. Immunology 134, 214-223, doi:10.1111/j.13652567.2011.03480.x (2011).

202 Pulendran, B., Tang, H. \& Manicassamy, S. Programming dendritic cells to induce $\mathrm{T}(\mathrm{H}) 2$ and tolerogenic responses. Nature Immunology 11, 647-655, doi:10.1038/ni.1894 (2010). 
203 Inaba, K., Inaba, M., Romani, N., Aya, H., Deguchi, M., Ikehara, S., Muramatsu, S. \& Steinman, R. M. Generation of large numbers of dendritic cells from mouse bone marrow cultures supplemented with granulocyte/macrophage colony-stimulating factor. J Exp Med 176, 1693-1702 (1992).

204 Naik, S. H., Proietto, A. I., Wilson, N. S., Dakic, A., Schnorrer, P., Fuchsberger, M., Lahoud, M. H., O'Keeffe, M., Shao, Q.-X., Chen, W.-f., Villadangos, J. A., Shortman, K. \& Wu, L. Cutting edge: generation of splenic CD8+ and CD8- dendritic cell equivalents in Fms-like tyrosine kinase 3 ligand bone marrow cultures. J Immunol 174, 6592-6597 (2005).

205 Kopf, M., Le Gros, G., Bachmann, M., Lamers, M. C., Bluethmann, H. \& Köhler, G. Disruption of the murine IL-4 gene blocks Th2 cytokine responses. Nature 362, 245-248, doi:10.1038/362245a0 (1993).

206 Ouyang, W., Löhning, M., Gao, Z., Assenmacher, M., Ranganath, S., Radbruch, A. \& Murphy, K. M. Stat6-independent GATA-3 autoactivation directs IL-4-independent Th2 development and commitment. Immunity 12, 2737 (2000).

207 McKenzie, G. J., Emson, C. L., Bell, S. E., Anderson, S., Fallon, P., Zurawski, G., Murray, R., Grencis, R. \& McKenzie, A. N. Impaired development of Th2 cells in IL-13-deficient mice. Immunity 9, 423-432 (1998).

208 Ohnmacht, C. \& Voehringer, D. Basophils protect against reinfection with hookworms independently of mast cells and memory Th2 cells. J Immunol 184, 344-350, doi:10.4049/jimmunol.0901841 (2010).

209 Wynn, T. A. IL-13 effector functions. Annu. Rev. Immunol. 21, 425-456, doi:10.1146/annurev.immunol.21.120601.141142 (2003).

210 Liang, H.-E., Reinhardt, R. L., Bando, J. K., Sullivan, B. M., Ho, I.-C. \& Locksley, R. M. Divergent expression patterns of IL-4 and IL-13 define unique functions in allergic immunity. Nature Immunology, doi:10.1038/ni.2182 (2011).

211 Barlow, J. L., Bellosi, A., Hardman, C. S., Drynan, L. F., Wong, S. H., Cruickshank, J. P. \& McKenzie, A. N. J. Innate IL-13-producing nuocytes arise during allergic lung inflammation and contribute to airways hyperreactivity. $J$ Allergy Clin Immunol, doi:10.1016/j.jaci.2011.09.041 (2011).

212 Finkelman, F. D., Holmes, J., Katona, I. M., Urban, J. F., Beckmann, M. P., Park, L. S., Schooley, K. A., Coffman, R. L., Mosmann, T. R. \& Paul, W. E. Lymphokine control of in vivo immunoglobulin isotype selection. Annu Rev Immunol 8, 303-333, doi:10.1146/annurev.iy.08.040190.001511 (1990).

213 Shinkai, K., Mohrs, M. \& Locksley, R. M. Helper T cells regulate type-2 innate immunity in vivo. Nature 420, 825-829, doi:10.1038/nature01202 (2002).

214 Zhu, Z., Homer, R. J., Wang, Z., Chen, Q., Geba, G. P., Wang, J., Zhang, Y. \& Elias, J. A. Pulmonary expression of interleukin-13 causes inflammation, mucus hypersecretion, subepithelial fibrosis, physiologic abnormalities, and eotaxin production. J Clin Invest 103, 779-788, doi:10.1172/JCI5909 (1999).

215 Barlow, J. L. \& McKenzie, A. N. J. Nuocytes: expanding the innate cell repertoire in type-2 immunity. Journal of Leukocyte Biology, doi:10.1189/jlb.0311160 (2011). 
216 Neill, D. R. \& McKenzie, A. N. J. Nuocytes and beyond: new insights into helminth expulsion. Trends in Parasitology, doi:10.1016/j.pt.2011.01.001 (2011).

217 Urban, J. F., Maliszewski, C. R., Madden, K. B., Katona, I. M. \& Finkelman, F. D. IL-4 treatment can cure established gastrointestinal nematode infections in immunocompetent and immunodeficient mice. J Immunol 154, 4675-4684 (1995).

218 Barlow, J. L., Flynn, R. J., Ballantyne, S. J. \& Mckenzie, A. N. J. Reciprocal expression of IL-25 and IL-17A is important for allergic airways hyperreactivity. Clinical \& Experimental Allergy, no-no, doi:10.1111/j.13652222.2011.03806.x (2011).

219 Ballantyne, S. J., Barlow, J. L., Jolin, H. E., Nath, P., Williams, A. S., Chung, K. F., Sturton, G., Wong, S. H. \& McKenzie, A. N. J. Blocking IL-25 prevents airway hyperresponsiveness in allergic asthma. J Allergy Clin Immunol 120, 1324-1331, doi:10.1016/j.jaci.2007.07.051 (2007).

220 Reece, J. J., Siracusa, M. C. \& Scott, A. L. Innate immune responses to lungstage helminth infection induce alternatively activated alveolar macrophages. Infection and Immunity 74, 4970-4981, doi:10.1128/IAI.00687-06 (2006).

221 Lajoie-Kadoch, S., Joubert, P., Létuvé, S., Halayko, A. J., Martin, J. G., Soussi-Gounni, A. \& Hamid, Q. TNF-alpha and IFN-gamma inversely modulate expression of the IL-17E receptor in airway smooth muscle cells. Am J Physiol Lung Cell Mol Physiol 290, L1238-1246, doi:10.1152/ajplung.00301.2005 (2006).

222 Hwang, E. S., White, I. A. \& Ho, I.-C. An IL-4-independent and CD25mediated function of c-maf in promoting the production of Th2 cytokines. Proc Natl Acad Sci USA 99, 13026-13030, doi:10.1073/pnas.202474499 (2002).

223 Perona-Wright, G., Mohrs, K., Mayer, K. D. \& Mohrs, M. Differential Regulation of IL-4R Expression by Antigen versus Cytokine Stimulation Characterizes Th2 Progression In Vivo. J Immunol 184, 615-623, doi:10.4049/jimmunol.0902408 (2010).

224 Sallusto, F., Mackay, C. R. \& Lanzavecchia, A. Selective expression of the eotaxin receptor CCR3 by human T helper 2 cells. Science 277, 2005-2007 (1997).

225 Bonecchi, R., Bianchi, G., Bordignon, P. P., D'Ambrosio, D., Lang, R., Borsatti, A., Sozzani, S., Allavena, P., Gray, P. A., Mantovani, A. \& Sinigaglia, F. Differential expression of chemokine receptors and chemotactic responsiveness of type 1 T helper cells (Th1s) and Th2s. J Exp Med 187, 129134 (1998).

226 Sallusto, F., Lenig, D., Mackay, C. R. \& Lanzavecchia, A. Flexible programs of chemokine receptor expression on human polarized $\mathrm{T}$ helper 1 and 2 lymphocytes. J Exp Med 187, 875-883 (1998).

227 D'Ambrosio, D., Iellem, A., Bonecchi, R., Mazzeo, D., Sozzani, S., Mantovani, A. \& Sinigaglia, F. Selective up-regulation of chemokine receptors CCR4 and CCR8 upon activation of polarized human type 2 Th cells. $J$ Immunol 161, 5111-5115 (1998).

228 Nagata, K., Tanaka, K., Ogawa, K., Kemmotsu, K., Imai, T., Yoshie, O., Abe, H., Tada, K., Nakamura, M., Sugamura, K. \& Takano, S. Selective expression of a novel surface molecule by human Th2 cells in vivo. J Immunol 162, 12781286 (1999). 
229 Löhning, M., Stroehmann, A., Coyle, A. J., Grogan, J. L., Lin, S., GutierrezRamos, J. C., Levinson, D., Radbruch, A. \& Kamradt, T. T1/ST2 is preferentially expressed on murine Th2 cells, independent of interleukin 4, interleukin 5, and interleukin 10, and important for Th2 effector function. Proc Natl Acad Sci USA 95, 6930-6935 (1998).

230 Xu, D., Chan, W. L., Leung, B. P., Huang, F. p., Wheeler, R., Piedrafita, D., Robinson, J. H. \& Liew, F. Y. Selective expression of a stable cell surface molecule on type 2 but not type 1 helper T cells. J Exp Med 187, 787-794 (1998).

231 Yasuda, K., Muto, T., Kawagoe, T., Matsumoto, M., Sasaki, Y., Matsushita, K., Taki, Y., Futatsugi-Yumikura, S., Tsutsui, H., Ishii, K. J., Yoshimoto, T., Akira, S. \& Nakanishi, K. Contribution of IL-33-activated type II innate lymphoid cells to pulmonary eosinophilia in intestinal nematode-infected mice. Proc Natl Acad Sci USA, doi:10.1073/pnas.1201042109 (2012).

232 Kay, A. B. Allergy and allergic diseases. First of two parts. N Engl J Med 344, 30-37, doi:10.1056/NEJM200101043440106 (2001).

233 Kay, A. B. Allergy and allergic diseases. Second of two parts. N Engl J Med 344, 109-113, doi:10.1056/NEJM200101113440206 (2001).

234 Gimelbrant, A., Hutchinson, J. N., Thompson, B. R. \& Chess, A. Widespread monoallelic expression on human autosomes. Science 318, 1136-1140, doi:10.1126/science.1148910 (2007).

235 Gett, A. V. \& Hodgkin, P. D. Cell division regulates the T cell cytokine repertoire, revealing a mechanism underlying immune class regulation. Proc Natl Acad Sci USA 95, 9488-9493 (1998).

236 Bird, J. J., Brown, D. R., Mullen, A. C., Moskowitz, N. H., Mahowald, M. A., Sider, J. R., Gajewski, T. F., Wang, C. R. \& Reiner, S. L. Helper T cell differentiation is controlled by the cell cycle. Immunity 9, 229-237 (1998).

237 Guo, L., Hu-Li, J. \& Paul, W. E. Probabilistic regulation of IL-4 production. J Clin Immunol 25, 573-581, doi:10.1007/s10875-005-8218-5 (2005).

238 Erazo, A., Kutchukhidze, N., Leung, M., Christ, A. P. G., Urban, J. F., Curotto De Lafaille, M. A. \& Lafaille, J. J. Unique maturation program of the $\operatorname{IgE}$ response in vivo. Immunity 26, 191-203, doi:10.1016/j.immuni.2006.12.006 (2007).

239 Kwan, M., Powell, D. R., Nachman, T. Y. \& Brown, M. A. An intron GATAbinding site regulates chromatin accessibility and is essential for IL-4 gene expression in mast cells. Eur. J. Immunol. 35, 1267-1274, doi:10.1002/eji.200425619 (2005).

240 Hural, J. A., Kwan, M., Henkel, G., Hock, M. B. \& Brown, M. A. An intron transcriptional enhancer element regulates IL-4 gene locus accessibility in mast cells. J Immunol 165, 3239-3249 (2000).

241 Harris, N. L., Watt, V., Ronchese, F. \& Le Gros, G. Differential T cell function and fate in lymph node and nonlymphoid tissues. J Exp Med 195, 317-326 (2002).

242 Poo, W. J., Conrad, L. \& Janeway, C. A. Receptor-directed focusing of lymphokine release by helper $\mathrm{T}$ cells. Nature 332, 378-380, doi:10.1038/332378a0 (1988).

243 Vitetta, E. S., Ohara, J., Myers, C. D., Layton, J. E., Krammer, P. H. \& Paul, W. E. Serological, biochemical, and functional identity of B cell-stimulatory factor 1 and B cell differentiation factor for IgG1. J Exp Med 162, 1726-1731 (1985). 
244 Ito, T., Wang, Y.-H., Duramad, O., Hori, T., Delespesse, G. J., Watanabe, N., Qin, F. X.-F., Yao, Z., Cao, W. \& Liu, Y.-J. TSLP-activated dendritic cells induce an inflammatory $\mathrm{T}$ helper type 2 cell response through OX40 ligand. $J$ Exp Med 202, 1213-1223, doi:10.1084/jem.20051135 (2005).

245 Rank, M. A., Kobayashi, T., Kozaki, H., Bartemes, K. R., Squillace, D. L. \& Kita, H. IL-33-activated dendritic cells induce an atypical TH2-type response. J Allergy Clin Immunol 123, 1047-1054, doi:10.1016/j.jaci.2009.02.026 (2009).

246 Besnard, A.-G., Togbe, D., Guillou, N., Erard, F., Quesniaux, V. \& Ryffel, B. IL-33-activated dendritic cells are critical for allergic airway inflammation. Eur. J. Immunol. 41, 1675-1686, doi:10.1002/eji.201041033 (2011).

247 Smith, D. E. IL-33: a tissue derived cytokine pathway involved in allergic inflammation and asthma. Clin Exp Allergy 40, 200-208, doi:10.1111/j.13652222.2009.03384.x (2010).

248 Kouzaki, H., O'Grady, S. M., Lawrence, C. B. \& Kita, H. Proteases induce production of thymic stromal lymphopoietin by airway epithelial cells through protease-activated receptor-2. J Immunol 183, 1427-1434, doi:10.4049/jimmunol.0900904 (2009).

249 Massacand, J. C., Stettler, R. C., Meier, R., Humphreys, N. E., Grencis, R. K., Marsland, B. J. \& Harris, N. L. Helminth products bypass the need for TSLP in Th2 immune responses by directly modulating dendritic cell function. Proc Natl Acad Sci USA 106, 13968-13973, doi:10.1073/pnas.0906367106 (2009).

250 Granucci, F., Feau, S., Angeli, V., Trottein, F. \& Ricciardi-Castagnoli, P. Early IL-2 production by mouse dendritic cells is the result of microbialinduced priming. J Immunol 170, 5075-5081 (2003).

251 Coffman, R. L., Seymour, B. W., Hudak, S., Jackson, J. \& Rennick, D. Antibody to interleukin-5 inhibits helminth-induced eosinophilia in mice. Science (New York, NY) 245, 308-310 (1989).

252 Suto, A., Nakajima, H., Hirose, K., Suzuki, K., Kagami, S.-I., Seto, Y., Hoshimoto, A., Saito, Y., Foster, D. C. \& Iwamoto, I. Interleukin 21 prevents antigen-induced $\operatorname{IgE}$ production by inhibiting germ line C(epsilon) transcription of IL-4-stimulated B cells. Blood 100, 4565-4573, doi:10.1182/blood-2002-04-1115 (2002).

253 Curotto de Lafaille, M. A., Muriglan, S., Sunshine, M. J., Lei, Y., Kutchukhidze, N., Furtado, G. C., Wensky, A. K., Olivares-Villagómez, D. \& Lafaille, J. J. Hyper immunoglobulin $\mathrm{E}$ response in mice with monoclonal populations of B and T lymphocytes. J Exp Med 194, 1349-1359 (2001).

254 Hepworth, M. R., Danilowicz-Luebert, E., Rausch, S., Metz, M., Klotz, C., Maurer, M. \& Hartmann, S. Mast cells orchestrate type 2 immunity to helminths through regulation of tissue-derived cytokines. Proc Natl Acad Sci USA, 1-10, doi:10.1073/pnas.1112268109 (2012).

255 Voehringer, D., Shinkai, K. \& Locksley, R. M. Type 2 immunity reflects orchestrated recruitment of cells committed to IL-4 production. Immunity 20, 267-277 (2004).

256 Crowle, P. K. \& Reed, N. D. Rejection of the intestinal parasite Nippostrongylus brasiliensis by mast cell-deficient $\mathrm{W} / \mathrm{Wv}$ anemic mice. Infect Immun 33, 54-58 (1981).

257 Sanders, V. M. Epigenetic regulation of Th1 and Th2 cell development. Brain Behav Immun 20, 317-324, doi:10.1016/j.bbi.2005.08.005 (2006). 
258 van Panhuys, N., Le Gros, G. \& McConnell, M. J. Epigenetic regulation of Th2 cytokine expression in atopic diseases. Tissue Antigens 72, 91-97, doi:10.1111/j.1399-0039.2008.01068.x (2008).

259 Brandt, E. B., Munitz, A., Orekov, T., Mingler, M. K., McBride, M., Finkelman, F. D. \& Rothenberg, M. E. Targeting IL-4/IL-13 signaling to alleviate oral allergen-induced diarrhea. J Allergy Clin Immunol 123, 53-58, doi:10.1016/j.jaci.2008.10.001 (2009).

260 Forbes, E. E., Groschwitz, K., Abonia, J. P., Brandt, E. B., Cohen, E., Blanchard, C., Ahrens, R., Seidu, L., McKenzie, A., Strait, R., Finkelman, F. D., Foster, P. S., Matthaei, K. I., Rothenberg, M. E. \& Hogan, S. P. IL-9- and mast cell-mediated intestinal permeability predisposes to oral antigen hypersensitivity. J Exp Med 205, 897-913, doi:10.1084/jem.20071046 (2008).

261 Chan, L. S., Robinson, N. \& Xu, L. Expression of interleukin-4 in the epidermis of transgenic mice results in a pruritic inflammatory skin disease: an experimental animal model to study atopic dermatitis. J Invest Dermatol 117, 977-983, doi:10.1046/j.0022-202x.2001.01484.x (2001).

262 Erb, K. J., Rüger, B., von Brevern, M., Ryffel, B., Schimpl, A. \& Rivett, K. Constitutive expression of interleukin (IL)-4 in vivo causes autoimmune-type disorders in mice. J Exp Med 185, 329-339 (1997).

263 Goren, A. \& Cedar, H. Replicating by the clock. Nat Rev Mol Cell Biol 4, 2532, doi:10.1038/nrm1008 (2003). 
